\title{
Availability-aware Secure Application Offloading for Mobile Edge Computing
}

by

He Zhu

A dissertation submitted to the Faculty of Graduate and Postdoctoral

Affairs in partial fulfillment of the requirements for the degree of

Doctor of Philosophy in Electrical and Computer Engineering

Ottawa-Carleton Institute for Electrical and Computer Engineering (OCIECE)

Department of Systems and Computer Engineering

Carleton University

Ottawa, Ontario

May, 2018

C) Copyright 2018, He Zhu 
The undersigned hereby recommends to the

Faculty of Graduate Studies and Research

acceptance of the dissertation

\title{
Availability-aware Secure Application Offloading for Mobile Edge Computing
}

\author{
submitted by \\ He Zhu, M.Eng \\ in partial fulfillment of the requirements for the degree of \\ Doctor of Philosophy in Electrical and Computer Engineering
}

Thesis Supervisor, Professor Changcheng Huang,

Department of Systems and Computer Engineering, Carleton University

Carleton University

May 2018 


\section{Abstract}

Mobile edge computing (MEC) literally pushes cloud computing to the life radius of end users. The state-of-the-art mobile edge applications (MEApps) are posing rigorous latency requirements to service providers, as well as the need of security and high availability. However, vulnerabilities exist for both cloud and MEC environments. In this dissertation, our goal is to create a secure MEC environment for hosting MEApps with high availability, and to encourage resource sharing by both users for their Customer-premises equipment (CPE) and service providers (SPs) for their MEC hosts. While there is a number of challenges to achieve the goal, we present four parts of work to address these challenges. We first investigate the security issues in offloading applications and analyze vulnerabilities by modules of an application. A model called EdgePlace is formulated as a stochastic programming problem with a heuristic algorithm to leverage affinity and anti-affinity host placement rules for higher availability and lower cost. We then present IoT-B\&B, an architecture featuring resource sharing of physical CPE nodes, with the goal to leverage unused resources at the network edge and to share them with users across the network edge. At last, we propose EdgeChain, a model for making fair MEApps placement decisions for multiple SPs and a heuristic placement algorithm for MEApps across different SPs. The algorithm is intended to run by multiple service providers for consensus and the placement decisions can be recorded onto a blockchain for the fairness of the results. 


\section{Acknowledgments}

I would like to express my greatest gratitude to my advisor, Prof. Changcheng Huang. He has been giving me guidance for how to do good research and what topics to choose for research. I have been always learning from him, not only for research, but also for the attitude facing events in my life. He always encourages me when I have hard time on my research. I would not be able to complete my thesis without his help.

As an important journey of my PhD study, I have spent two years at InBay Technologies Inc., where I picked up much industrial experience and the way of problem thinking in a more practical and tangible manner. I truly appreciate the experience that gave me the chance to meet the great people working for the small company. I would like to thank Stan Xavier, Randy Kuang, and Robert Steklasa for their great leadership to the company and for their kind help on both my study and my life.

It has been my pleasure working with the faculty, staff, and students at Carleton University during the long journey of being a $\mathrm{PhD}$ student. I have met many other excellent students and researchers there who have similar goals and interests. True happiness comes from day-to-day work and discussions on the common topics we are interested in. I would like to thank Zhexiong Wei, Wenda Ni, Yegui Cai for their advices to my past work.

At last, I would like to appreciate the strong and unconditional support from my wife Yiwen Wang and our families. Thank you for being patient and for always being supportive to my $\mathrm{PhD}$ study. 
Dedicated to my wife Yiwen Wang who made all of this possible for her endless encouragement and patience 


\section{Table of Contents}

Abstract

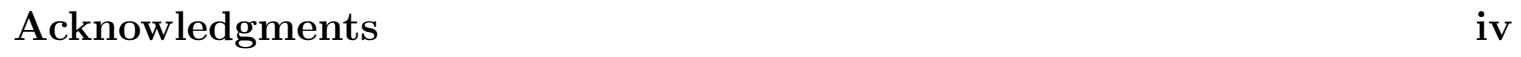

Table of Contents vi vi v

List of Tables $\quad$ xii

List of Figures $\quad$ xiii

List of Abbreviations $\quad$ xvii

List of Symbols $\quad$ xix

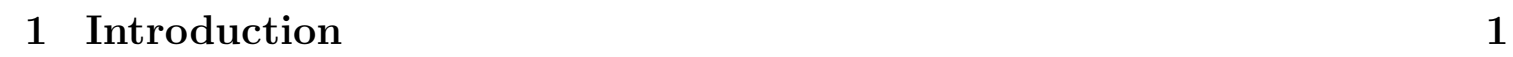

1.1 Motivations . . . . . . . . . . . . . . . . . . . . . . . . . . 1

1.2 Problem Statement And Challenges . . . . . . . . . . . . . . . . . . . 2

1.2.1 Security in Offloading Applications . . . . . . . . . . . . 2

1.2 .2 Service Availability in Offloading to the Edge . . . . . . . . . 3

$1.2 .3 \quad$ Resource Sharing at the Edge with CPE . . . . . . . . . . . . 4

1.2 .4 Multi-vendor Resource Sharing . . . . . . . . . . . . . . . . . 6

1.3 Contributions . . . . . . . . . . . . . . . . . . . . . 7

1.4 Dissertation Organization $\ldots \ldots \ldots \ldots$. . . . . . . . . . 8 
1.4 .1 List of Publications . . . . . . . . . . . . . . . . 9

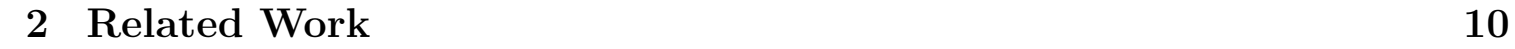

2.1 Security Issues in Cloud Computing . . . . . . . . . . . . . . . 10

2.1 .1 Deployment and Service Models . . . . . . . . . . . . . . 11

2.1 .2 Distinctive Characteristics $\ldots \ldots \ldots \ldots$

$2.1 .3 \quad$ Security Requirements . . . . . . . . . . . . . . . . 14

2.1 .4 The CIA triad of Cloud Security $\ldots \ldots \ldots \ldots$

2.2 Cloud-specific Vulnerabilities and Threats $\ldots \ldots \ldots \ldots$

2.2 .1 Vulnerabilities and Threats in IaaS . . . . . . . . . . 17

2.2 .2 Vulnerabilities and Threats in PaaS . . . . . . . . . . . 22

2.2 .3 Vulnerabilities and Threats in All Service Models . . . . . . . 24

$2.2 .4 \quad$ Vulnerabilities and Threats in Mobile Cloud Computing . . . 25

2.3 Methods of Defense and Remaining Issues $\ldots \ldots \ldots \ldots \ldots$

2.3 .1 Defending DoS Attacks to Cloud Systems $\ldots \ldots \ldots \ldots$

$2.3 .2 \quad$ Inhibiting Side-channel Attacks $\ldots \ldots \ldots \ldots \ldots$

$2.3 .3 \quad$ Ensuring Safe Authentication and Encryption $\ldots \ldots \ldots$

2.3 .4 Protecting the Privacy $\ldots \ldots \ldots \ldots$

$2.3 .5 \quad$ Risk Configuration and Profile Assessment . . . . . . . . . . 31

$2.3 .6 \quad$ Securing Mobile Cloud Computing $\ldots \ldots \ldots \ldots$. . . . . . 32

$2.4 \quad$ Cloud-assisted Mobile Computing and Its Vulnerabilities . . . . . . . 34

$2.5 \quad$ Job Partitioning Between Clouds and Mobile Devices . . . . . . . . 36

$2.5 .1 \quad$ Application-level Partitioning Based on VM Images . . . . . . 37

$2.5 .2 \quad$ Module level partitioning . . . . . . . . . . . . . . 38

$2.6 \quad$ Scheduling and Optimization in Job Partitioning . . . . . . . . . . . 39

2.7 Securing Cloud-assisted Mobile Computing . . . . . . . . . . . . . . 39

2.8 NFV Placement, Resource Sharing and Blockchain . . . . . . . . 41 
2.8 .1 Blockchain and Resource Allocation . . . . . . . . . . . . . . . 45

$\begin{array}{lll}3 & \text { Vulnerability For Offloaded Applications } & 46\end{array}$

3.1 Introduction . . . . . . . . . . . . . . . . . . . . 46

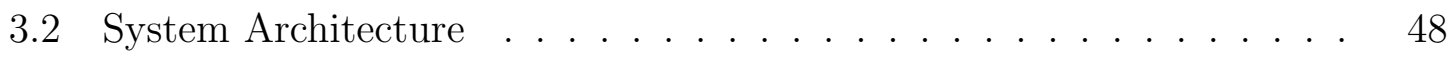

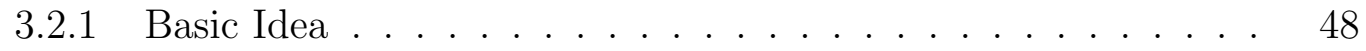

3.2 .2 System Modules . . . . . . . . . . . . . . . . . . . . . . . . 49

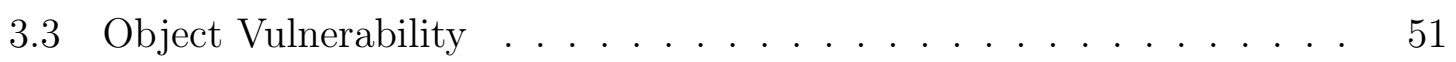

3.3.1 Call Relationship and Message Passing . . . . . . . . . . . . 51

3.3 .2 Propagated Vulnerabilities . . . . . . . . . . . . . . . . . 53

3.3.3 Cloud-originated Vulnerabilities . . . . . . . . . . . . . 54

3.3 .4 Object Vulnerabilities . . . . . . . . . . . . . 55

3.4 Object Dependency Graph . . . . . . . . . . . . . . . . 55

$3.4 .1 \quad$ Vulnerability Propagation Model in ODGs . . . . . . . . . . . 57

3.4 .2 The Impact of Offloading on Object Vulnerability . . . . . . . 63

3.5 Evaluation and Numerical Results . . . . . . . . . . . . . . . . . . 65

3.5.1 Standard Process of Experiments . . . . . . . . . . . . 68

3.5 .2 Experimental Results . . . . . . . . . . . . . . . . . 69

3.6 Chapter Summary . . . . . . . . . . . . . . . . . . 73

\begin{tabular}{|ll|}
\hline & Availability-aware Application Placement \\
\hline
\end{tabular}

4.1 Introduction . . . . . . . . . . . . . . . . . . . . . . . 74

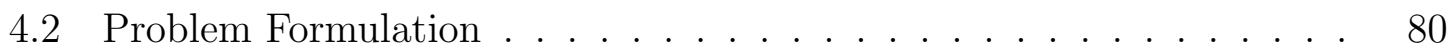

$4.2 .1 \quad$ Availability of Elastic Mobile Edge Applications . . . . . . . . 80

$4.2 .2 \quad$ Inter-host Link Availability and Importance Factor . . . . . . 83

4.2 .3 Inter-host Network Bandwidth Costs . . . . . . . . . . . . . . 84

$4.2 .4 \quad$ CPU and Memory Costs . . . . . . . . . . . . . 86 
4.2 .5 Cloud Costs . . . . . . . . . . . . . . . . . . . . . . 88

$4.2 .6 \quad$ Stochastic Programming . . . . . . . . . . . . . . . . . . . . 89

$4.2 .7 \quad$ Stochastic Programming Formulation $\ldots \ldots \ldots$. . . . . . 89

4.2 .8 Scalability and the EdgePlace Algorithm . . . . . . . . . . 92

4.3 Numerical Results. . . . . . . . . . . . . . . . . . . . . . . . . . . . . . 95

$4.3 .1 \quad$ Assumptions $\ldots \ldots \ldots \ldots \ldots$

4.3 .2 Parameters . . . . . . . . . . . . . . . . . . 97

$4.3 .3 \quad$ Availability Impact from Number of Instances and Hosts . . . 98

4.3 .4 Latency . . . . . . . . . . . . . . . . . . . . . . . . . . 101

$4.3 .5 \quad$ Availability Impact from SFC . . . . . . . . . . . . . . 103

4.3 .6 Distribution of VMs with Different Bandwidth Costs _. . . . 103

4.4 Chapter Summary . . . . . . . . . . . . . . . . . . . . . . . . . . 104

$\begin{array}{lll}5 & \text { CPE Resource Sharing At The Edge } & 105\end{array}$

5.1 Introduction . . . . . . . . . . . . . . . . . . . . 105

5.2 Problem Formulation . . . . . . . . . . . . . . . . . . . . . 108

5.2 .1 Connected pCPE at Network Edge . . . . . . . . . . 108

5.2 .2 VNF Types and Resource Requirement Profiles . . . . . . . 110

5.2 .3 The User of a VNF instance . . . . . . . . . . . . . . . . . 111

$5.2 .4 \quad$ Places to Deploy VNF instances . . . . . . . . . . . . . . . 111

$5.2 .5 \quad$ Factors to Impact Placement Decisions $\ldots \ldots \ldots \ldots \ldots$

5.2 .6 Cost of Offloading to Edge Network $\ldots \ldots \ldots \ldots \ldots$

5.2 .7 Cost of Offloading to Cloud $\ldots \ldots \ldots \ldots \ldots \ldots$

5.2 .8 Objective and 0-1 Integer Programming Formulation $\ldots . . .119$

$5.3 \quad$ IoT-B\&B Heuristic Placement Algorithm . . . . . . . . . . . . . 123

5.3 .1 Preliminary Resource Check . . . . . . . . . . . . . . . . . 123

5.3 .2 Cost Estimation . . . . . . . . . . . . . . . . . . . 123 
5.3 .3 Time Complexity . . . . . . . . . . . . . . . . . . . . . 124

5.4 System Implementation $\ldots \ldots \ldots$

5.4 .1 Hardware Configuration of pCPE Nodes . . . . . . . . 126

$5.4 .2 \quad$ NFVI Setup . . . . . . . . . . . . . . . . . . . . . . 126

5.4 .3 IoT-B\&B Algorithm As Filter Scheduler . . . . . . . . . . 127

$5.4 .4 \quad$ System Life Cycle. . . . . . . . . . . . . . . . . . . . . . . . 129

5.4 .5 Typical Use Cases $\ldots \ldots \ldots$

5.5 Numerical Results. . . . . . . . . . . . . . . . . . . . . . . . . . . . . 131

$5.5 .1 \quad$ Host Nodes Setup . . . . . . . . . . . . . . . . . . . . . 132

5.5 .2 VNF Resource Requirement Profile (Flavor) Types . . . . . . 134

5.5 .3 Placement Configuration Modes . . . . . . . . . . . . . . . 134

$5.5 .4 \quad$ Extended VNF Instance Capacity . . . . . . . . . . . . . . 134

$5.5 .5 \quad$ Cost Hike By Cloud Load Increase $\ldots \ldots \ldots$

$5.5 .6 \quad$ Impact from Outside the Network Edge. . . . . . . . . . . 137

5.5 .7 Remaining Resource Levels $\ldots \ldots \ldots$

5.6 Chapter Summary . . . . . . . . . . . . . . . . . . . . . . . . . . . . . . . 139

\begin{tabular}{|lll}
\hline 6 & Multi-vendor Edge Resource Sharing & 140 \\
\hline
\end{tabular}

6.1 Introduction . . . . . . . . . . . . . . . . . . . . . . . . . 140

6.2 Problem Formulation . . . . . . . . . . . . . . . . . . . . . . . 143

6.2 .1 HostLink Unit Price . . . . . . . . . . . . . . . . 146

6.2 .2 HostLink latency . . . . . . . . . . . . . . . . . . . . 148

6.2 .3 Integer Programming Formulation . . . . . . . . . . . 148

6.3 The EdgeChain Placement Algorithm . . . . . . . . . . . . . 150

6.3 .1 Processing Order and selection of MEHosts. . . . . . . . 150

6.4 Numerical Results. . . . . . . . . . . . . . . . . . . . . . . . . . . . 153

6.4 .1 Parameters $\ldots \ldots \ldots \ldots \ldots \ldots \ldots$ 
6.4 .2 Placement trends with changing unit resource premium . . . . 154

$6.4 .3 \quad$ Placement trends with changing user distribution . . . . . . . 155

6.5 EdgeChain Design and Implementation . . . . . . . . . . . . . . . . . 156

6.5 .1 Data Entities . . . . . . . . . . . . . . . 157

6.5 .2 EdgeChain Work Flow . . . . . . . . . . . . . . . . . . 159

6.6 Chapter Summary . . . . . . . . . . . . . . . . . . 161

\begin{tabular}{|lll}
\hline 7 & Conclusions and Future Work & 162
\end{tabular}

7.1 Conclusions . . . . . . . . . . . . . . . . . . . . . . . 162

7.2 Future work . . . . . . . . . . . . . . . . . . . . . 163

\begin{tabular}{ll}
\hline List of References & 165
\end{tabular}

\begin{tabular}{|ll}
\hline Appendix A Confidence interval & 177
\end{tabular} 


\section{List of Tables}

2.1 Cloud models and security responsibilities by providers and users . . 14

3.1 Parameters of sample apps . . . . . . . . . . . . . . . . 66

3.2 Time taken for analyzing apps with the three offloading mechanisms . $\quad 70$

$4.1 \quad$ Pre-defined hardware requirement profiles for simulation. . . . . . . . 97

4.2 Parameters for availability-aware host selection . . . . . . . . . . . . 98

5.1 Constant configurations . . . . . . . . . . . . . . . . 132

5.2 Predefined flavor types for simulation . . . . . . . . . . . . . . . 133

6.1 Parties involved in a MEC placement scenario . . . . . . . . . . . . . 143

6.2 Parameters for the MEC scenario . . . . . . . . . . . . . . . . . . . . 154

A.1 $\quad$ T distribution table (one-tail, partial) . . . . . . . . . . . . . . 179 


\section{List of Figures}

$2.1 \quad$ Security is the highest rated issue to adopt to cloud . . . . . . . . . . 11

$2.2 \quad$ Shared technologies cause cloud-specific vulnerabilities and threats . . 17

$3.1 \quad$ System architecture of the vulnerability-based offloading framework . $\quad 49$

3.2 Three types of call relationships . . . . . . . . . . . . . . . . . 50

3.3 Initiator attacks and observer attacks . . . . . . . . . . . . . . . . 52

3.4 An ODG with $M_{o d}=4 \ldots \ldots$. . . . . . . . . . . . . . . 60

3.5 Comparison between the actual value of $p_{o} d$ and its upper bound. $\alpha=$ 0.5 and $p_{e} \in[0,0.2] \ldots \ldots \ldots$. . . . . . . . . . . . . . . . 63

3.6 ODG of TrendCraw after the first piece of news has been retrieved. . 64

$3.7 \quad$ ODG of TrendCraw fetching with only original objects . . . . . . . . 64

$3.8 \quad$ ODG generation process on existing open-source Android apps . . . . 68

3.9 Vulnerabilities of three objects under different cloud-originated vulnerabilities $(\alpha)$ and propagated vulnerabilities $(\beta)$. . . . . . . . . . 69

3.10 Number of one- and two-hop neighbors may impact the sensitivity of objects to system-wide vulnerability change . . . . . . . . . . . . . . 71

3.11 Normalized phone-side energy cost with the three offloading schemes under different percentages of objects offloaded . . . . . . . . . . . . . 72

3.12 Numbers of sensitive APIs accessible by the cloud . . . . . . . . . . . 73

$4.1 \quad$ A mobile edge application deployment with host placement rules. . . 76

4.2 An attempt to migrate a service chain without enough resource. . . . 77 
4.3 Inefficient partitioning of VMs for a mobile edge application. . . . . . 78

4.4 Inter-host and intra-host traffic between two VMs . . . . . . . . . . . 86

4.5 A service graph consisting of 3 service chains . . . . . . . . . . . . . . 94

4.6 Availability of the mobile edge application with different numbers of VMs deployed on various numbers of mobile edge hosts using the EdgePlace algorithm. Confidence level is $95 \%$. . . . . . . . . . . . . . . . 99

4.7 Availability of the mobile edge application with different numbers of VMs deployed on various numbers of mobile edge hosts using the stochastic model . . . . . . . . . . . . . . . . . . . 100

4.8 Availability of the mobile edge application with varying minimum number of VMs required and different numbers of mobile edge hosts. Confidence level is $95 \%$. . . . . . . . . . . . . . . . . . . . . . 100

4.9 Average latency of the mobile edge application with different numbers of VMs deployed on remote cloud and various numbers of hosts . . . 101

4.10 Availability of an application consisting of $6 \mathrm{VMs}$ with $95 \%$ confidence level and four hardware requirement profiles (F1, F4, F7, and F10) 102

4.11 Placement distribution of $20 \mathrm{VMs}$ across 10 hosts with $N_{H}=10, N_{V}=$ $20, y=2, W_{C}=W_{M}=1$, and $\eta=0.3 \%$. . . . . . . . . . . . . . . 104

5.1 The system architecture extended from ETSI NFV architecture . . . 107

5.2 Topologies at network edges $\ldots \ldots$. . . . . . . . . . . . . . . 109

$5.3 \quad$ An example of VNF instances grouped by $u_{1} \ldots \ldots$. . . . . . . . . . . 111

5.4 Capacities of vCPUs and memory of a pCPE node . . . . . . . . . . 113

5.5 Time complexity comparison between IoT-B\&B algorithm and an exhaustive algorithm . . . . . . . . . . . . . . . . . . . . . . 125

$5.6 \quad$ IoT-B\&B service backed by container-based OpenStack . . . . . . . . 127

5.7 IoT-B\&B algorithm in OpenStack as a filter scheduler . . . . . . . . . 128 
5.8 IoT-B\&B service system life cycle . . . . . . . . . . . . . . . . . . 128

5.9 Initial resource levels of the 100 nodes used for experiments . . . . . . 132

5.10 Total number of VNF instances deployed for the network edge with various flavors and placement modes and 95\% confidence level . . . . 135

5.11 Cost hikes when the cloud load increases in Local+Cloud Mode with 95\% confidence level . . . . . . . . . . . . . . . . . . 135

5.12 Cost hikes when the cloud load increases in Local+Cloud $+B \varepsilon B$ Mode with $95 \%$ confidence level . . . . . . . . . . . . . . . . . . . 136

5.13 Cost changes when load increases due to tasks outside the target network edge with $95 \%$ confidence level . . . . . . . . . . . . . . . . . . 137

5.14 Remaining resource levels after all B\&B nodes used . . . . . . . . . . 138

$6.1 \quad$ A MEC scenario in a certain service area with 3 ME base stations from

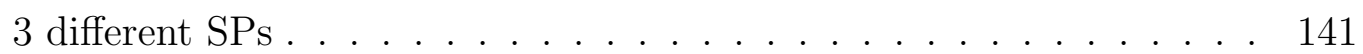

6.2 Placement results of 3 service chains consisting of 15 MEApps in all . 154

6.3 Numbers of MEApps placed on the 3 MEHosts with different percentages of users in the network with $95 \%$ confidence level . . . . . . . . 155

6.4 Data entities and their relationship used by and stored in EdgeChain, including MECSPs, MEHosts, HostLinks, SvcChains, MEApps, and AppLinks. . . . . . . . . . . . . . . . . . 157

6.5 Typical work flow of EdgeChain. MECSPs, MEAVs, and mining nodes participate in the process. Steps of the work flow are marked by circled numbers and alphabets with details documented in Section 6.5.2. . . 159 
6.6 A placement transaction in EdgeChain. A state transition happens upon a transaction. As this figure shows, $M E A p p-4$ owned by $M E A V$ 6 is to be placed with the requirement of 2 vCPUs and $4096 \mathrm{MB}$ of memory. The input of the EdgeChain placement algorithm is the current state of the two MEHosts and $M E A V-6$. The result is to place MEApp-4 onto MEHost-2. After the transaction is accepted, the resources taken by $M E A p p-4$ are deducted from the remaining resources of MEHost-2. MEAV-6 will pay tokens as fees to MEHost-2 for hosting the service. . . . . . . . . . . . . . . . . . . . . . . 161

A.1 Estimated availability values versus stopping time. For getting the example when the system is in stable state, the stopping time needs to be greater than 200 seconds . . . . . . . . . . . . . . . . . . . . . 178 


\section{List of Abbreviations}

AOP Aspect-oriented programming

AppLink Application link

AR Augmented reality

$\mathrm{CE} \quad$ Customer edge

CPE Customer-premises equipment

CS Critical security

DDoS Distributed denial-of-service

DoS Denial-of-service

EPC Evolved packet core

HA High availability

HostLink Host link

IA Implicit authentication

IaaS Infrastructure as a service

IoT Internet of things

ILP Integer linear programming

ME Mobile edge

MEApps Mobile edge application

MEAV Mobile edge application vendors

MEC Mobile edge computing 


$\begin{array}{ll}\text { MECSP } & \text { MEC service provider } \\ \text { MEHosts } & \text { MEC host } \\ \text { MVNO } & \text { Mobile virtual network operator } \\ \text { NFV } & \text { Network function virtualization } \\ \text { NS } & \text { Normal security } \\ \text { NSC } & \text { Network service chaining } \\ \text { ODG } & \text { Object dependency graph } \\ \text { OOP } & \text { Object-oriented programming } \\ \text { OPEX } & \text { Operating expense } \\ \text { OVS } & \text { Open vSwitch } \\ \text { PaaS } & \text { Platform as a service } \\ \text { pCPE } & \text { Physical customer-premises equipment } \\ \text { PoC } & \text { Proof of concept } \\ \text { QEMU } & \text { Quick emulator } \\ \text { SaaS } & \text { Software as a service } \\ \text { SFC } & \text { Service function chaining } \\ \text { SP } & \text { Service provider } \\ \text { TSP } & \text { Telecommunication service provider } \\ \text { UE } & \text { User equipment } \\ \text { vCPE } & \text { Virtual customer-premises equipment } \\ \text { VM } & \text { Virtual machine } \\ \text { VNF } & \text { Virtual Network Function } \\ \text { XSS } & \text { Cross site scripting }\end{array}$




\section{List of Symbols}

\section{Symbols used in Chapter 3}

$V, E$

$o, d$

$A_{o o}, p_{o o}$

$m, r_{o d}^{m}$

$A_{o d}^{m}, p_{o d}^{m}$

$E_{d}, P\left\{E_{d}\right\}$

$\mu_{d}, \pi_{d}$

$R_{o d}, C_{o d}$
$V$ is the set of all objects in an app. $E$ is the set of undirected edges representing all call relationships between objects in $V$.

$o$ is the originating node of an attack. $d$ is the destination node of the attack.

$A_{o o}$ is the event of the originator $o$ being compromised for its cloud-originated vulnerability. $p_{o o}$ as the probability of $A_{o o}$ to occur.

$m$ denotes the index of the path the attack takes from $o$ to the target object $d$. $r_{o d}^{m}$ denotes the attack path.

$A_{o d}^{m}$ is the event that $o$ has incurred a cloud-originated attack and the attack has propagated to $d$ along $r_{o d}^{m}$. $p_{o d}^{m}$ denote the probability of $A_{o d}^{m}$ to occur.

$E_{d}$ denotes the event that $d$ is compromised for any reason. $P\left\{E_{d}\right\}$ denotes the object vulnerability of $d$.

$\mu_{d}$ is the propagated vulnerability of $d . \pi_{d}$ is the object vulnerability of $d$.

$R_{o d}$ is the set of all minimal paths between $o$ and $d . C_{o d}$ is the minimal sets of edges whose failure of vulnerability propagation ensure the failure of the attack propagating from $o$ to $d$. 


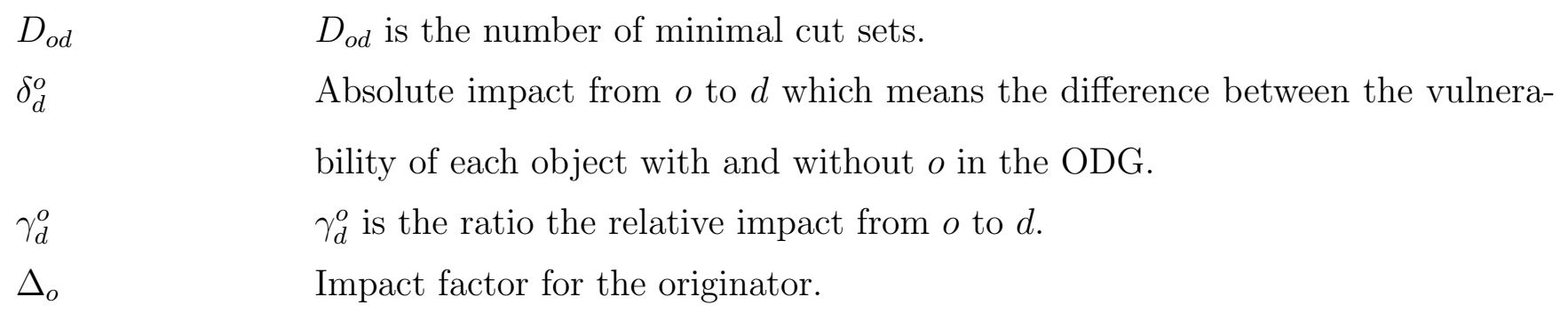

\section{Symbols used in Chapter 4}

$\mathbb{V}, N_{V}, v$

$\mathbb{H}, N_{H}, h$

$c$

$e_{i j}, a_{i j}$

$x_{v h}, x_{v c}$

$N_{m}$

$P_{V}, P_{H}$

$P_{E}\left(e_{i j}\right)$

$\hat{v}_{h}, \hat{v}_{c}$

$A_{h}, p_{a_{h}}$

$p_{\bar{a}}$

$p_{E}(\bar{a})$

$\mathbb{V}$ is the set of $N_{V}$ VMs provisioning a VNF, each VM denoted by $v$.

$\mathbb{H}$ is the set of $N_{H}$ hosts available for VNF deployment, each host denoted by $h$.

$c$ stands for the remote cloud location.

$e_{i j}$ is the network link between the two hosts $h_{i}$ and $h_{j} . a_{i j}$ is the maximum number of virtual links possible on $e_{i j}$.

If $v$ is deployed on $h$, then $x_{v h}=1$. Otherwise, $x_{v h}=0$. If $v$ is deployed on the cloud, then $x_{v c}=1$. Otherwise, $x_{v c}=0$.

Minimum number of active VMs required by the VNF.

$P_{V}$ is the probability of a $\mathrm{VM}$ working without internal failure. $P_{H}$ is the probability of a host not failing.

$P_{E}\left(e_{i j}\right)$ is the probability that $e_{i j}$ is up and available.

Total number of VMs assigned to host $h$ and the cloud $c$.

$A_{h}$ is the random variable of the number of VMs available on $h . p_{a_{h}}$ is the probability there are $a_{h}$ VMs available on $h$.

Probability there are $\left(a_{1}, a_{2}, \ldots, a_{H}\right)$ VMs on hosts $\left(h_{1}, h_{2}, \ldots, N_{H}\right)$, given $\bar{a}=$ $\left(a_{1}, a_{2}, \ldots, a_{H}\right)$.

Probability all hosts with one or more VMs deployed are connected to each other. 


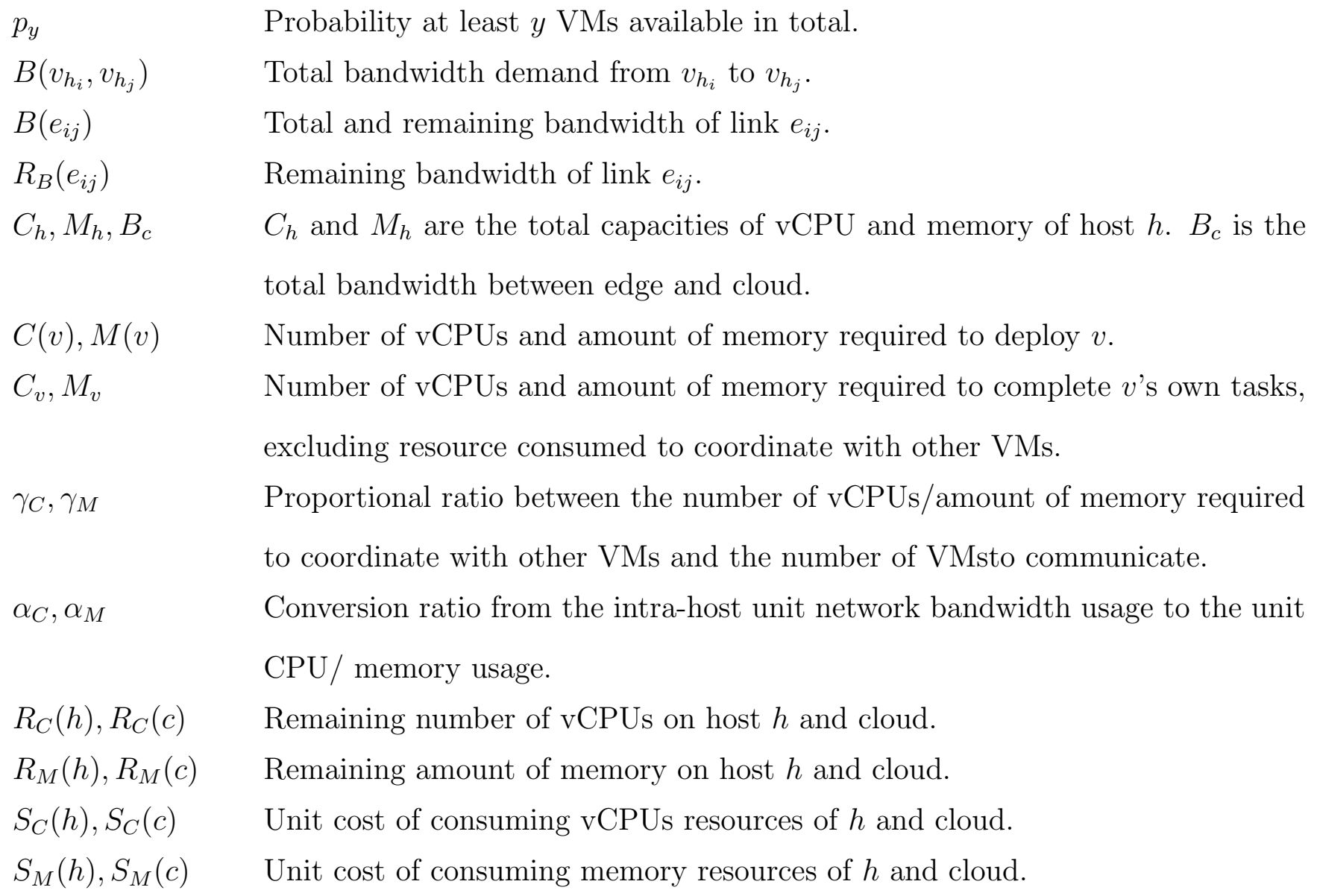

\section{Symbols used in Chapter 5}

$v, v_{i}, n_{V} \quad v$ is a pCPE node. $v_{i}$ is a specific pCPE node by its index, where $i \in\left[1 . . n_{V}\right]$. $n_{V}$ is the total number pCPE nodes in the network edge.

$u, u_{i} \quad u$ is a user. Each user owns one pCPE node. $u_{i}$ is a specific user by the pCPE index, where $i \in\left[1 . . n_{V}\right]$.

$l_{i j}, l_{c}, R\left(l_{c}\right) \quad l_{i j}$ is the link between pCPE node $v_{i}$ and $v_{j}$, where $i, j \in\left[1 . . n_{V}\right], i \neq j . l_{c}$ is the link between the network edge and the core network. $R\left(l_{c}\right)$ is the remaining network bandwidth of $l_{c}$. 


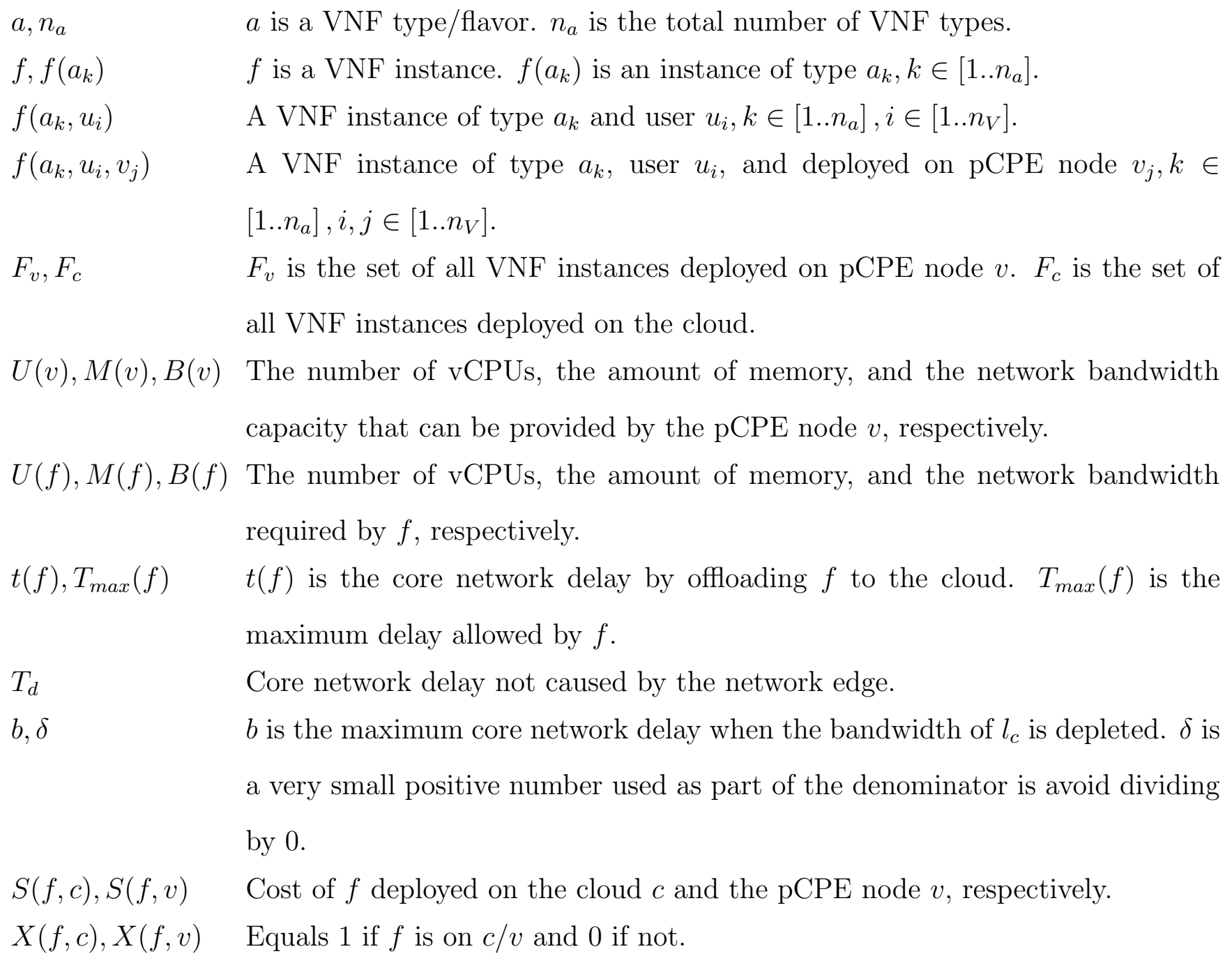

$U(f), M(f), B(f)$ The number of vCPUs, the amount of memory, and the network bandwidth required by $f$, respectively.

$t(f), T_{\max }(f) \quad t(f)$ is the core network delay by offloading $f$ to the cloud. $T_{\max }(f)$ is the maximum delay allowed by $f$.

$T_{d} \quad$ Core network delay not caused by the network edge.

$b, \delta \quad b$ is the maximum core network delay when the bandwidth of $l_{c}$ is depleted. $\delta$ is a very small positive number used as part of the denominator is avoid dividing by 0 .

$S(f, c), S(f, v) \quad$ Cost of $f$ deployed on the cloud $c$ and the pCPE node $v$, respectively.

$X(f, c), X(f, v) \quad$ Equals 1 if $f$ is on $c / v$ and 0 if not.

\section{Symbols used in Chapter 6}

$s, \mathbb{V}_{s}, \mathbb{L}_{s}, v, l \quad s$ is a service chain. $\mathbb{V}_{s}$ is the set of all MEApps in $s . \mathbb{L}_{s}$ is the set of all AppLinks in $s$. A MEApp in $s$ is denoted by $v \in \mathbb{V}_{s}$, and an AppLink between two MEApps in $s$ is denoted by $l \in \mathbb{L}_{s}$. 
$\mathbb{H}, \mathbb{E}, h, e, \mathbb{V}_{h} \quad \mathbb{H}$ is the set of all MEHosts. $\mathbb{E}$ is the set of all HostLinks. A MEHost is denoted by $h \in \mathbb{H}$, and a link between two MEHosts is denoted by $e \in \mathbb{E}$. $\mathbb{V}_{h}$ is the set of all MEApps placed on $h$.

$u, m, c_{s} \quad u$ is an end user. $m$ is a MECSP. $c_{s}$ is the cost of deploying $s$.

$c_{v}, c_{v h_{i}, v^{\prime} h_{j}} \quad c_{v}$ is the cost of deploying $v \cdot c_{v h_{i}, v^{\prime} h_{j}}$ is the cost of the AppLink between $v$ on $h_{i}$ and $v^{\prime}$ on $h_{j}$.

$n_{s}, P_{m}, h_{m} \quad n_{s}$ is the total number of users requesting $s . P_{m}$ is a random variable denoting the percentage of the users of MECSP $m . h_{m}$ is an edge host of $m$.

$\gamma_{m}, \delta_{m}$ $\gamma_{m}$ is the unit price of serving $m$ 's own subscribers. $\delta_{m}$ is the extra charge for $m$ serving users of other MECSPs.

$C_{v}, M_{v} \quad$ CPU and memory requirement of the MEApp $v$.

$B\left(e_{i j}\right), \zeta_{e_{i j}} \quad B\left(e_{i j}\right)$ is the total bandwidth capacity of HostLink $e_{i j} . \zeta_{e_{i j}}$ is the unit price of the bandwidth of $e_{i j}$.

$B_{V}\left(e_{i j}\right) \quad B_{V}\left(e_{i j}\right)$ is the total bandwidth used by MEApps deployed on $h_{i}$ and $h_{j}$.

$B\left(v_{h_{i}}, v_{h_{j}}\right) \quad$ Bandwidth used between MEHosts $h_{i}$ and $h_{j}$.

$C_{h}, M_{h} \quad \mathrm{CPU}$ and memory capacity of the MEHost $h$.

$t_{e_{i j}}, t_{s}, T_{s} \quad t_{e_{i j}}$ is the latency incurred on HostLink $e_{i j} . t_{s}$ is the latency of the service chain s. $T_{s}$ is the max latency allowed by $s$. 


\section{Chapter 1}

\section{Introduction}

\subsection{Motivations}

Cloud computing provides computational resources with high availability and reasonable prices. State-of-the-art mobile apps, such as facial recognition, image and video processing, have increased the consumption of on-demand computational resources. Therefore, offloading apps to have them run on cloud servers has emerged [1 4$]$ as an attractive solution in order to exploit the capacity and efficiency of cloud computing, as well as saving limited power of mobile devices and accelerate the speed of executing resource-intensive applications.

As more applications start to have higher latency requirements, mobile edge computing (MEC) and Customer-premises equipment (CPE) devices are also getting attentions and attractions in the industry. MEC is taking Network Function Virtualization (NFV) to the end users closer than ever [5 7]. Instances of edge computing, including regional datacenters [8], cloudlets [1], and fog nodes [9], deliver highlyresponsive cloud services at the network edge. As a key technology towards $5 \mathrm{G}$, MEC architecture proposed by ETSI [7] leverages existing NFV frameworks widely adopted by carriers and vendors $[10,11]$. Elastic mobile edge applications, including 
network services, are deployed close to the user equipment (UE) with low latency. Both UE application providers and telecommunication service providers (TSPs) can take advantage of MEC to reduce cost and to adjust services with agility based on fastchanging user demands. As a supplement to the MEC-enable base stations deployed by the service providers (SPs), CPE devices, such as routers, switches, residential gateways, and set-top boxes, have been deployed at the subscriber's premises to originate, route and terminate communications between the customer premises and the central office. In the wake of cloud computing and NFV [12] [13], service providers leverage virtual Customer-premises Equipment (vCPE) as Virtual Network Function (VNF) instances on top of generic physical Customer-premises Equipment (pCPE), in search of rebuilding a dynamic revenue stream. There can be enough resources for pCPE to deploy VNFs locally [14], while pCPE can also coordinate with the cloud if VNF scale-out is needed to accommodate heavier usage.

\subsection{Problem Statement And Challenges}

When an application is offloaded to the cloud, it becomes a cloud-based application. In comparison, when an application is offloaded to the network edge, it is then considered as a mobile edge application. In order to be securely offloaded, both cloud and mobile edge applications face problems to be resolved. We state the problems to resolve in the following section. This dissertation focuses on the following problems in application offloading to the cloud and the network edge.

\subsubsection{Security in Offloading Applications}

One critical issue to resolve in offloading apps is how to manage the security risks of cloud computing [15, 16] and MEC. Many mobile applications use and store personal 
information related to banking, health, business, messaging and so on. Sensitive information sent to the remote cloud or MEC hosts has a good chance to be exposed to either service providers or malicious customers who have access to the same hardware [16].

Traditional ways to protect remote execution and data storage include mutual authentication, authorization and data encryption for the whole app. If every single part of the app was armored by well-prepared security protections, the system would be safe enough but the cost would also rocket up and not be acceptable for commercial use, because too much information has to be encrypted unnecessarily [17]. The redundant encryption and decryption operations hurt both energy efficiency and user experience. We need to consider the trade-offs between security and usability, and to maximize the system security subject to a tolerable delay and resource cost.

\subsubsection{Service Availability in Offloading to the Edge}

A mobile edge application consists of one or more collaborating virtual machines (VMs). It is of paramount importance to maintain the high availability of mobile edge applications. Compared to centralized datacenters used by public cloud, MEC hosts are heterogeneous with varying computing, storage and networking capabilities [18]. Smaller scale private cloud servers can be deployed near their designated groups of users as MEC hosts, leading to problems specific in MEC environments caused by less reliable hosts with lower availability: A single MEC server deployment is less powerful compared to the highly-available, centralized cloud as it serves a smaller group of users within the base station's coverage; the offloading nature of MEC brings higher system complexity that can jeopardize the availability [19]; service function chaining (SFC) 20] is possible on MEC servers, as videos, augmented reality data, location-based services, and other computational-intensive tasks can take a chain of 
services to process.

To maximize the availability while maintaining costs and latencies at acceptable levels, placement rules often come into play to tune the performance and security of a mobile edge application [21]. In practice, placement rules mainly refer to the affinity and anti-affinity rule [22]. A group of VMs with the affinity rule applied must be deployed on the same host. On the contrary, the anti-affinity rule to a group of VMs ensures that each VM in the group is deployed on a different host. The affinity rule helps reduce communication costs between VMs serving the same mobile edge application but it brings lower availability caused by less host redundancy. In comparison, anti-affinity rules are ideal for High Availability (HA) with higher host redundancy, while it increases communications costs between VMs.

Besides hosts availability, SFC is commonly adopted to formulate a network function with complete features to provide end-to-end service. Enforcing SFC will make the migration of the service more difficult and add more restrictions to the placement strategy.

Considering placement constraints of host availability, SFC, and the limited resources at the mobile edge, it can be foreseen that when mobile edge hosts are unavailable, mobile edge application VMs might not be able to continue service if they are kept at the edge. To maintain the desired availability, the more reliable, centralized cloud can come to the picture to coordinate with the edge.

\subsubsection{Resource Sharing at the Edge with CPE}

Taking advantage of centralized cloud services in the core networks has benefits 23 because of scalable and flexible computing capabilities. However, large-number deployments of Internet-of-Things (IoT) devices bring challenges to VNFs running in a 
centralized cloud, as the network traffic load would be drastically increased by transmitting data between the core and the edge of the network. Such traffic overhead can become unacceptable with excessive data transmission, causing high processing delay or even service outage due to the congestion of the network. Meanwhile, high usage of the cloud networks would jack up the price per usage, resulting in higherthan-expected operating expense (OPEX).

Recent research has been aware of the explosive growth of devices in the edge of the networks. The concept of fog computing [24] and edge computing [5] were proposed to move the initial handling of raw data to the edge for IoT devices. Although the fog can mitigate the load of the core network, the power of the Customer Edge (CE), namely the computational capabilities of $\mathrm{CPE}$, is buried. While a single pCPE node has limited resources and typically serves a designated location, the aggregated computing capabilities of pCPE nodes across the edge of a network can be powerful: pCPE nodes have time-varying resource usage that does not always reach full workloads. For instance, the home gateways typically have significantly lower usage in business hours as their users leave for work, while office gateways become idle in after hours. If the spare resources of pCPE can be shared within the network edge, VNFs will be able to roam around the edge. Both SP and users will benefit from the considerable capabilities of the sharable resources. Meanwhile, the VNFs deployed on the pCPE nodes keep most traffic within the edge and reduce the traffic to the core network.

It certainly sounds interesting to utilize the time-varying computational resources. However, CPE resource sharing faces challenges. First, service availability becomes a concern. A pCPE node's availability can be jeopardized if it no longer has enough resources to host VNFs. It can also be down due to power outages or user pulling the plugs. The availability of offloaded VNFs must be ensured by enforcing proper redundancy. Second, the users need to be motivated to consent contributing their 
pCPE nodes for resource sharing. Incentives returned to users are required in order to benefit both the SP and its end users.

\subsubsection{Multi-vendor Resource Sharing}

For encouraging SPs to enroll their eligible MEC base stations and hosts in resource sharing, it is common to give incentives to SPs for contributing their resources of the hosts for hosting edge applications. Following the changing demand of end users, certain types of edge applications need to be deployed on, migrated to, or removed from an edge host, in order to meet the service requirement. By deploying the edge applications at the right places, the edge application provider will save costs, while providing high-quality service with low latency to the end users. Meanwhile, the edge host will collect incentives for its resources effectively used.

Clearly, the edge computing framework needs a placement service to dynamically check the user needs and the available edge hosts, and determine the placement or removal of edge applications. In datacenters, VM placement has been well investigated, mainly with the focus of more efficient resource utilization and lower OPEX. However, the collaboration of multiple SPs and mobile edge applications vendors are posing new challenges.

A placement model is required to make transparent and consistent selections of the best host for each request for edge computing resources, along with a trusted party to determine the best place for application deployment. Besides, the application placement service needs to be steadily available. Both the mobile edge hosting service providers and the mobile edge application providers can constantly change. The placement service provider must remain in service regardless of the joining or quitting of vendors. 


\subsection{Contributions}

In this dissertation, we have four major parts of contributions illustrated below.

We first propose a graph-based analytical model called object dependency graph (ODG), by dividing an app into multiple parts and connect them by their dependencies. The vulnerability of each part may propagate along its dependencies and can be evaluated by our model. The vulnerability level of all app parts can be evaluated by our model to make offloading decisions jointly so that cloud resources can be best utilized, while the security level is not violated. An algorithm based on the ODG model is designed available for popular smartphone operating systems like Android.

Then we focus on finding adaptive placement strategies for different types of mobile edge applications to achieve lower costs, while still satisfying the availability and confidentiality requirements. The application availability concerns are addressed from the following perspectives: the link health between hosts is considered to support SFC to migrate VMs when links are down. A cost model is then built considering the factors of inter-host traffic and resource overcommitting, following a stochastic programming problem formulation to minimize the cost based on our cost model, while maintaining the availability requirements. A heuristic algorithm, namely EdgePlace, is developed to return suboptimal results as the problem scales.

Next, we present an architecture to support resource sharing of pCPE nodes. A model is proposed to evaluate the cost of assigning a VNF instance to a pCPE node and to the remote cloud. Multiple factors are considered to determine the cost, including remaining resources, network transmission delay, and availability requirements. A placement algorithm called IoT-B\&B Algorithm is also presented to for assign vCPE instances to $\mathrm{pCPE}$ nodes with the goal to find a cost-efficient pCPE node for each VNF. The chapter also shows the implementation of a system with the IoT-B\&B architecture with steps to setup the NFVI and the system's life cycle. 
At last, an architecture combined with a heuristic algorithm, namely EdgeChain, is proposed to create placement model leverage by multiple SPs for mobile edge application that does not require trust to any party, i.e., trustless placement service. A cost model first is presented as a stochastic programming problem, factoring in the pricing of edge hosts, latency, and service chaining. Then a heuristic placement algorithm is developed based on the proposed cost model, with the consideration of storing placement decisions to a blockchain. Simulation results of our placement algorithm show its effectiveness in mobile edge host resource sharing among SPs.

\subsection{Dissertation Organization}

This dissertation splits the work for a secure and highly available MEC environment into four chapters according to our major contributions listed in the previous section, each with the focus of a specific area.

Chapter 2 first covers related work with the introduction on cloud security, security threats in cloud computing and current solutions. Then it lists existing work on improving availability of MEC and solutions of resource sharing at the network edge. Chapter 3 is dedicated for our first series contribution of the ODG modeling and the vulnerability evaluation algorithm. The availability-aware mobile edge application placement model and algorithm are then presented in Chapter 4. The resource sharing model and architecture named IoT-B\&B are covered in Chapter 5 . The work of EdgeChain with the focus on resource sharing of multiple SPs with the

least trust requirements are presented in Chapter 6. Finally, Chapter 7 concludes the dissertation. 


\subsubsection{List of Publications}

The following accepted or submitted papers are partially covered in this dissertation. H. Zhu, C. Huang, and J. Yan, Vulnerability Evaluation for Securely Offloading Mobile Apps in the Cloud, in Proceedings of IEEE International Conference on Cloud Networking 2013 (CloudNet), San Francisco, Nov. 2013.

H. Zhu and C. Huang, Cost-Efficient VNF Placement Strategy for IoT Networks with Availability Assurance, in IEEE 86th Vehicular Technology Conference (VTC-Fall), December 4-8, 2017, Singapore.

H. Zhu and C. Huang, Availability-aware Mobile Edge Application Placement in 5G Networks, in IEEE Globecom 2017, December 4-8, 2017, Singapore.

H. Zhu and C. Huang, EdgePlace: Availability-aware Placement For Chained Mobile Edge Applications, submitted to Transactions on Emerging Telecommunications Technologies, Wiley.

H. Zhu and C. Huang, VNF-B\&B: Enabling Edge-based NFV with CPE Resource Sharing, in IEEE 28th Annual International Symposium on Personal, Indoor, and Mobile Radio Communication (PIMRC), October 8-13, 2017, Montreal.

H. Zhu and C. Huang, IOT-B\&B: Edge-Based NFV for IoT Devices with CPE Crowdsourcing, Wireless Communications and Mobile Computing, vol. 2018, Article ID 3027269, 15 pages, 2018. doi:10.1155/2018/3027269.

H. Zhu, C. Huang, and J. Zhou, EdgeChain: Blockchain-based Multi-vendor Mobile Edge Application Placement, IEEE 4th International Conference on Network Softwarization, June 25-29, 2018, Montreal. 


\section{Chapter 2}

\section{Related Work}

This chapter provides a review of the literature related to this dissertation.

\subsection{Security Issues in Cloud Computing}

Cloud computing is a widely-adopted technology today that have been benefiting every end user. With the concept of cloud computing, the computation resources can be accessed and charged as a utility like electricity or tap water. Users only need to pay for what they actually used, therefore saving budgets on buying or updating perspective, cloud computing has the ability to integrate the resources together, enabling better utilization to achieve lower costs. Such affordable services have attracted a great number of potential customers, preparing to move their platform into the cloud.

However, the security issues, which can be taken as the byproduct brought by the shared technologies of cloud computing, are becoming a serious problem we cannot ignore. Most customers are sensitive to the security of their systems more than the cost. According to the survey conducted by IDC shown in Figure 2.1, the highest rated issue about cloud computing service is security: with their business information and critical IT resources outside the firewall, customers worry about their vulnerabilities 
and chances of being attacked [25]. They will not move in until the cloud security has been more convincing.

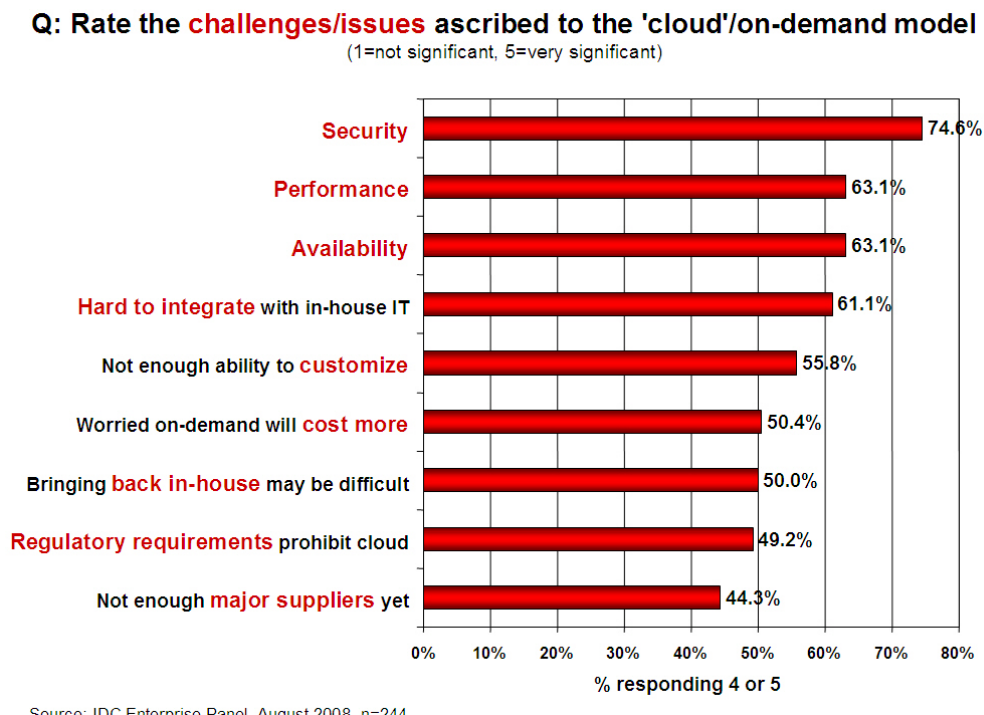

Figure 2.1: Security is the highest rated issue to adopt to cloud

\subsubsection{Deployment and Service Models}

Cloud computing can be divided into the following categories based on how cloud infrastructures are deployed:

- Private cloud

- Community cloud

- Public cloud

- Hybrid cloud

Private cloud is used by only one organization. For example, a cloud created and maintained by a company. If multiple organizations which have cooperation and trust, they can share the service provided by a community cloud. Different departments 
of the government can use the same community cloud. Public cloud usually has a powerful computation capacity and will sell their service as a utility to everyone who needs. That is to say, different users even including competitors can use the same public cloud. Hybrid cloud is the combination of either two or three kinds of the clouds above.

According to the services cloud infrastructures provide, cloud computing can also be divided into Infrastructure as a Service (IaaS), Platform as a Service (PaaS), and Software as a Service (SaaS).

IaaS is the foundation of cloud services and provides infrastructure resources in the form of VMs. Amazon EC2 is one well-known IaaS platform. PaaS is built on IaaS and meanwhile forms the groundwork of SaaS. Users can interact with PaaS by invoking the interfaces and APIs it provides. For example, Google App Engine enables users creating their websites with PHP APIs. SaaS directly gives users online software. Customers use such software as they did in their traditional desktop machines. Todays online ERP systems are good examples of SaaS.

Just as other capabilities, for the upper layer services, the features of the security architecture are inherited from the lower layer ones. If the users use services of higher layers, they can expect the service providers take care of the security for the lower layers. However, this will add up the dependency to the service providers for the users. In a word, the lower layer users control, the more responsibility they have to take for the security management.

\subsubsection{Distinctive Characteristics}

Cloud computing has the following distinctive characteristics which make them different from traditional client-server architectures. They should also be the ultimate causes of cloud-specific security issues. 
On-demand self-service. A consumer can unilaterally provide computing capabilities, such as server time and network storage, as needed automatically without requiring human interaction with each service provider. Creating a server in Amazon EC2 is as simple as online shopping. Customers only need to provide their credit card information and then select services fit for them.

Broad network access. Capabilities are available over the network and accessed through standard mechanisms that promote use by heterogeneous thin or thick client platforms (e.g., mobile phones, tablets, laptops, and workstations).

Resource pooling. The providers computing resources are pooled to serve multiple consumers using a multi-tenant model, with different physical and virtual resources dynamically assigned and reassigned according to consumer demand. There is a sense of location independence in that the customer generally has no control or knowledge over the exact location of the provided resources but may be able to specify location at a higher level of abstraction (e.g., country, state, or datacenter). Examples of resources include storage, processing, memory, and network bandwidth.

Rapid elasticity. Capabilities can be elastically provisioned and released, in some cases automatically, to scale rapidly outward and inward commensurate with demand. To the consumer, the capabilities available for provisioning often appear to be unlimited and can be appropriated in any quantity at any time. The rapid elasticity allows customers to change the scale of their services as they need. For instance, the job requiring 1000 hours computation with one server can be finished in 1 hour with 1000 servers at the same price. This amazing elasticity will save a lot of time especially for gusty large-scale computation.

Measured service. Cloud systems automatically control and optimize resource use by leveraging a metering capability at some level of abstraction appropriate to the type of service (e.g., storage, processing, bandwidth, and active user accounts). 
Resource usage can be monitored, controlled, and reported, providing transparency for both the provider and consumer of the utilized service.

\subsubsection{Security Requirements}

It is of great importance to have a deep understanding of the relations and dependencies between different cloud service models. The concept of infrastructure security refers to the security of the core infrastructure at the network, host and application levels.

Table 2.1: Cloud models and security responsibilities by providers and users

\begin{tabular}{ccccc}
\hline $\begin{array}{c}\text { Cloud } \\
\text { Model }\end{array}$ & $\begin{array}{c}\text { Provider } \\
\text { Control }\end{array}$ & $\begin{array}{c}\text { Provider } \\
\text { Responsibilities }\end{array}$ & $\begin{array}{c}\text { User } \\
\text { Control }\end{array}$ & $\begin{array}{c}\text { User } \\
\text { Responsibilities }\end{array}$ \\
\hline SaaS & High & $\begin{array}{c}\text { Confidentiality } \\
\text { Integrity } \\
\text { Availability }\end{array}$ & Low & None \\
PaaS & Medium & Integrity & Medium & $\begin{array}{c}\text { Confidentiality } \\
\text { Data Privacy }\end{array}$ \\
& & Availability & & Confidentiality \\
IaaS & Low & Availability & High & Data Privacy \\
& & & & Integrity \\
\hline
\end{tabular}

According to the operational layer users currently focus on, there are various categories of security issues. Sorted by the layer they are related to, we list these categories in Table 2.1. From the table we can learn that users take least responsibility in SaaS and take most in IaaS. Higher degree of control by users helps with service customization. And the vulnerabilities of services created by users increase as well. We mainly focus on IaaS Security analysis because the resource quantification is available in IaaS while it is not in neither PaaS nor SaaS. 


\subsubsection{The CIA triad of Cloud Security}

The CIA triad means Confidentiality, Integrity and Availability, which are the three fundamental tenets of information security. All of the information security controls and safeguards, and all of the threats, vulnerabilities, and security processes are subject to the CIA yardstick [26]. Every kind of vulnerability is potential to cause a violation to the CIA triad.

\section{Integrity}

Integrity stands for the property whereby an entity has not been modified in an unauthorized manner, guarding against improper information modification or destruction, and includes ensuring information non-repudiation and authenticity [27]. The concept of cloud information integrity requires that the following three principles are met 26]. In cloud computing, the integrity of messages and data should be protected first. Otherwise, the messages will be faked and cannot be trusted. It is the prerequisite of confidentiality and availability. Strong authentication technologies should be used to protect integrity in clouds.

\section{Confidentiality}

According to the definition in [27], confidentiality is the property that information is not disclosed to system entities (users, processes, devices) unless they have been authorized to access the information. It preserves authorized restrictions on information access and disclosure, including means for protecting personal privacy and proprietary information. Encryption methods have been used to ensure the confidentiality of the cloud. The characteristic of the traffic should also be hidden in case of traffic analysis by eavesdroppers. 


\section{Availability}

Availability represents the property of being accessible and usable upon demand by an authorized entity, ensuring timely and reliable access to and use of information [27]. In cloud computing, it means (i) to guarantee the hardware available as long as possible. The service providers and users usually have SLA agreements which guarantee the lowest up time percentage of the services. (ii) when being attacked, the users need to try the best to keep the service running.

\subsection{Cloud-specific Vulnerabilities and Threats}

As we stated before, cloud security becomes most critical to every organization who is interested in migrating to cloud computing platforms. Before doing any work on cloud computing security, it is necessary to know why cloud computing is not secure and where these insecure issues come from.

Cloud computing systems have vulnerabilities as other information systems do, making both service providers and customers suffer from data loss or leakage, service outage and other unexpected incidents. For every kind of vulnerability, there exist a few kinds of threats, which are able to exploit that vulnerability. Some of the vulnerabilities also exist in non-cloud systems, while others are cloud-specific. They are caused by the unique characteristics of cloud systems. In this section, we focus on cloud-specific vulnerabilities and threats.

Based on the general introduction of cloud computing, the shared technologies used by cloud computing systems are the most significant difference comparing with traditional systems. For different service models, shared technologies lead to different vulnerabilities and threats as illustrated in Figure 2.2. Following these characteristics of cloud-specific vulnerabilities, a detailed list can be generated below. In connection 


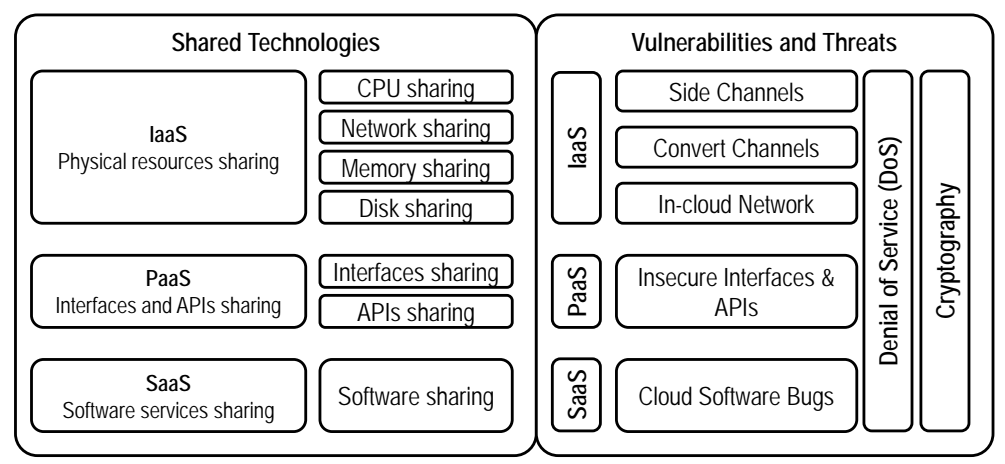

Figure 2.2: Shared technologies cause cloud-specific vulnerabilities and threats

with these vulnerabilities, we can also find the threats they are resulting in.

\subsubsection{Vulnerabilities and Threats in IaaS}

IaaS service providers use virtualization technologies to provide infrastructure services for multiple users from different organizations. Virtualization technologies are one of the most important technologies enabling cloud computing as it supports multitenancy, running multiple virtual machines in a single physical server. Although the virtualization technologies pay much attention on service isolation for different users, the logically isolated virtual machines are still running on the same physical server. They may use the same CPU core in different cycles, transmitting and receiving data with the same network card, and take up the memory blocks with the same addresses at different time. Shared technologies enable the vulnerabilities for IaaS, which vary according to what and how the resources are shared. Now we discuss the vulnerabilities and corresponding threats of sharing different resources in IaaS.

\section{Side Channels and Covert Channels Vulnerabilities}

The resource sharing of multiple virtual machines allows passive observations between correlated VMs, enabling side channels between VMs. The VMs have access to shared resources and thus can do various kinds of measurement including CPU cache load, 
network bandwidth utilization, etc.

CPU Cache Load Measurement. VMs sharing the same CPU have side channels and can measure the cache load of CPU on the physical machine of the VM. A measurement method called Prime+Trigger+Probe was introduced [16] to support measuring the CPU load of time-shared virtual machines. There were three steps to generate each load sample: (i) read buffer B at s-byte offsets to make sure it is cached, where $\mathrm{s}$ is the cache line size in bytes; (2) Busy loop until CPUs cycle counter jumps by a large value which should be the VM preempted by the VM scheduler; (3) measure the time it takes to again read B at s-byte offsets. And the final result would be the CPU cycle numbers which are strongly related to cache load.

Covert Channel Based On Load Measurement. The cache load measurement leads to effective covert channels between cooperative processes in different VMs. This also works when network is unavailable. For instance, the sender process in a VM transmitted 1 by frantically accessing the memory and 0 by keeping idle [28]. The receiver could get the bit by periodically measuring the latency accessing shared memory. Apparently higher latency means the activities of the sender which stands for 1 . And vise versa. The covert channels could be used for communications between the attackers and agents. The monitors in the cloud would not detect this activity. So it is a powerful tool to schedule DDoS attacks. We can imagine a cloud with many VMs compromised by attackers. Even if the connection to the Internet is cut, agents still can use covert channels to organize attacks and cause high load by accessing resources at the same time. Another example is the L2 cache covert channel threat explored by Xu et. al [29]. They provided a quantification of the bit rates of a L2 cache-based channel and an assessment of its ability to do harm. By assuming the leakage information as the credit card number, the covert channel with too slow bitrate is useless to attackers because it takes more than a month to get just one card 
number. By assessing the bit-rates of covers channels from ideal models to real ones progressively, adding aspects in consideration like hardware specification, workloads, hypervisor configuration and protocol design, the bit-rates are lower than ideal ones. But it is still proved to be usable for attackers.

Shared Network Side Channels. It is also possible to create side channels with shared network between different VMs. The network utilization can be measured by any VM sharing the same network device. Take SSH for example. The utilization revealed sensitive information such as the length of user password by timing information [30]. By using advanced statistical techniques on timing information collected by the network traffic monitor, the users typing pattern could be learned which revealed the information of the keys typed. The input sequence could even be predicted by a Hidden Markov Model. The timing method was also used to perform traffic analysis to determine the role of a target machine.

\section{In-cloud Network Vulnerabilities}

There are up to tens of thousands of physical servers and even more virtual machines in a cloud. Despite of the connection to the Internet through the firewalls, the virtual machines form a private network itself. This private network is large due to the number of virtual machines. However, the machines in this network do not belong to the same customer or organization. This causes various vulnerabilities for cloud computing.

IP address leakage. Although the network addresses are divided into multiple parts of subnets for different organizations, there is still a chance to get numerically close IP address ranges using WHOIS queries [16]. For Amazon EC2, after the queries, the IP addresses related to EC2 are found and these IP addresses can be translated to internal IP addresses. The leakage of internal IP address may lead to the exposure 
of the network topology for a certain organization, because network administrators usually follow the rules of internal IP address assignment.

Instance placement leakage. The cloud service providers prefer to allocate different IP ranges for different availability zones and instance types [16]. This has been proven in EC2 by launching multiple instances to exploit the IP assignment rules. Combined with the IP address leakage before, it is not hard to infer the availability and type of the target instance through appropriate statistics. As long as purchasing the same availability and type, it is possible for the attackers to be assigned to the place nearby.

Under-provisioned datacenter networks. The entity of in-cloud networks is the datacenter networks. In such scenarios, a router is typically connected to a large numbers of hosts, making the bandwidth to uplinks under-provisioned. This opens a potential venue for exploit. An adversary can saturate the bandwidth of a network to perform the Denial-of-Service (DoS) attack to other applications in this network 31. What is worse, the network firewalls usually do not set strict rules to machines in the same private network. This vulnerability makes the attackers have the chance to enter the same private network only by purchasing the same infrastructure service. With more ports exposed to the attackers, it is much easier to compromise the target machines.

\section{DoS Attack}

Even if the resources are scalable, the cloud services can still be attacked by receiving and responding to vast number of messages which may consume one or more key resources of the target. For example, an online booking system would be out of service to legitimate users if it receives millions of booking requests sent by attackers which are related to database transaction and cost a lot of CPU and memory resources. 
There are two conditions for implementing a successful DoS attack. First, the attackers must send requests as same as legitimate ones. Otherwise, malicious requests will be denied by the targets after proper filters have been set. Second, there must be large enough requests to deplete the resources of the victims. For this reason, multiple machines are usually required by attackers to generate distribute requests. This type of attack can be called Flooding Attack or Distributed DoS (DDoS) Attack.

Traditional DDoS attacks are of many forms to attack different types of victims, including application, host, resource, network and infrastructure. The differences between cloud servers and traditional one are mainly from resource and network factors.

Resource attacks in cloud systems. The multi-tenancy environment and sidechannel vulnerabilities enable attackers to exploit the capacity of target VMs, find their bottleneck, and then perform very efficient attacks causing serious damage with least cost. Two steps need to be done to attack resources.

The first step is to build side channels and exploit the activity and utilization of the resources. One example is monitoring CPU usage and sending a certain amount of requests. After enough groups of attempts with different request loads when observing the change of CPU usage, the maximum capacity of requests for CPU can be calculated. The second step is to attack the target in the cloud. The interesting thing in clouds is that in-cloud network is also quite large, allowing machines to be attacked from inside the cloud. This vulnerability increases the threat of being attacked greatly. Comparing with traditional systems, the network topology has a good chance to expose when the machines in the cloud is compromised. The in-cloud network gives the opportunities of network topology discovery to every machine in it. VMs are able to use Ping, traceroute, SNMP and other tools to get the network topology. Besides, another approach can be used to detect the network topology, 
which exploits the multiplexing nature of a router [31].

The powerful computation resources could not only benefit the users but also the attackers. Breaking into a cloud and compromising machines are just the first step of these attacks. The next step is to utilize the resources of the victim cloud can perform DDoS attacks as we described before to the targets outside the cloud. The consequences are seriously bad for both the agent cloud and other victims outside the cloud. For other victims, they suffer from huge amount of distributed requests because the agent cloud has thousands of machines and unique IP addresses. For the

agent cloud itself, most resources have been consumed by attackers, thus may violate SLAs for its customers. On the other hand, as an agent performing massive attacks to other organizations, they are very likely to be blacklisted by other organizations, affecting legitimate users, too.

\subsubsection{Vulnerabilities and Threats in PaaS}

The PaaS services allow the users to create their services using programming interfaces or APIs, without worrying about infrastructures. That is to say, the users are no longer in control of physical resources. As a result, the vulnerabilities in IaaS are replaced by the ones related to PaaS sharing, which come from the interfaces and APIs facing to both users and attackers. The interfaces or APIs can get part or even full control to the services of the users because they are related to everything users can do. If such interfaces are vulnerable, the whole chain of the services could be compromised by malicious attackers. Apparently the probability that unauthorized access could occur is much higher than that for traditional systems where the management functionality is accessible only by a few administrators. 


\section{Message Modification Attack}

If a signature of a message can be reused or can be faked, the attackers can generate a message by themselves and then attach the old or faked signature. Once the target accepts the message, malicious operations are on the way. A security analysis was provided on the control interfaces of a large Public Cloud [31]. The results showed that the control interfaces could be compromised with novel signature wrapping and advanced Cross Site Scripting (XSS) technologies. Knowledge of a single signed SOAP message was sufficient to attain a complete compromise of the security within the customer's account because one could generate arbitrary SOAP messages accepted by this interface from only one valid signature. To make things even worse, in one attack variant, knowledge of the (public) X.509 certificate alone enabled a successful execution of an arbitrary cloud control operation on behalf of the certificate owner. Those included actions such as starting or stopping virtual machines, downloading or uploading virtual machine images, resetting the administrator's password for cloud instances, and so on. Moreover, a persistent XSS vulnerability was found that allowed an adversary to perform an automated attack targeted at stealing user name or password data from EC2/S3 customers. The APIs are designed and used in all of the three service models. So this vulnerability also exists in all models. Macintosh et al. also pointed out in [32] that naive use of XML Signature might result in signed documents remaining vulnerable to undetected modification by an adversary.

Issues about insecure Interfaces and APIs are often related to authorization, authentication and audits. These problems also exist in traditional platforms without cloud computing technologies. In this proposal, we will not focus on this kind of issues because it mostly depends on the safety of the platform. 


\section{Vulnerabilities and Threats in SaaS}

SaaS is ready to provide users with software running in clouds. Users only access the functions provided by the software, or customize the software with the predefined range. This type of service model gives users least possibility to personalize the software, while it is the most convenient and fastest way to deploy a system for most ordinary users. For example, users who want to create a document can use Google Doc after registering a Google Account. They can edit their documents in this cloud software. But when they need to do view their bank account, they have to start another program rather than just customize the software. Similar as in PaaS, the vulnerabilities mainly come from those of the software itself. When a bug is found in cloud software, i.e., Google Doc, all the users will be affected by that bug and therefore result in more serious threats in the cloud. On the other hand, fixing the bug is relatively more efficient. Once the bug is fixed in the cloud, it is fixed for all the users. This issue will not be focused on in this proposal, either.

\subsubsection{Vulnerabilities and Threats in All Service Models}

There are vulnerabilities commonly existing in all service models of cloud computing. The related technologies of them are commonly used by IaaS, PaaS and SaaS. The virtually unlimited computing, network and storage resources of cloud computing give possibilities launching different scales of applications they want. Unfortunately, there is abuse and nefarious use of cloud computing among the users. They can build these malicious applications either by themselves or by attackers after their services got compromised, including spam servers, botnets and other illegal applications based on both PaaS and IaaS. 


\section{Cryptography}

Cryptography is used commonly in cloud computing services, because it is the only choice when the private data of users have to be stored and transmitted in a public or third-party environment. They are used in all service models with different forms such as RSA and AES. The messages between users and the correlated services and data storing in the cloud should be encrypted to prevent them from unauthorized accesses. They are considered as the last protection to the private data.

Cryptanalysis advances render any cryptographic mechanism or algorithm insecure as novel methods of breaking them are discovered. Its even more common to find crucial flaws in cryptographic algorithm implementations, which can turn strong encryption into weak encryption (or sometimes no encryption at all). Because broad uptake of cloud computing is unthinkable without the use of cryptography to protect data confidentiality and integrity in the cloud, insecure or obsolete cryptography vulnerabilities are highly relevant for cloud computing.

On the other hand, cryptography also consumes computing resources as a serious of algorithms. The price for safety is more time and more budgets. If we need to maximize the privacy, we have to encrypt everything, leading to a considerable cost and taking much more time than usual. If the service requires low latency and low computational costs, the range of applying cryptography should be considered carefully.

\subsubsection{Vulnerabilities and Threats in Mobile Cloud Comput- ing}

Cloud computing for mobile devices is becoming a more and more important topic since the wide deployment of related applications. Apples iCloud synchronization system is a good example. It can make backup for everything in the mobile devices to 
the cloud server, including application profiles, photos, configurations, etc. Backberry messenger and email services from Research In Motion also rely on cloud services because the company takes Amazon EC2 as their email and messenger servers. In spite of the cloud specific vulnerabilities and threats above, we should specially pay attention to the issues which are sensitive in mobile cloud computing environment.

User data leakage. The personal data such as photos and configurations from different users is stored together, making it possible for malicious users or attackers to compromise a small part of the cloud and then get a block of data which contains information from many users.

Mobile user traffic analysis. Mobile devices can use Internet service either from service operators or from WiFi connections. The connection to cloud servers will also work in untrustful data service, which may have the risk to be eavesdropped by attackers. By collecting information from multiple WiFi access points, attackers have the ability to gain the communication mechanism of the synchronization applications with cloud servers. This is helpful for understanding the transmission protocol, blocking the services or causing data burst at the same time for a lot of mobile clients.

DDoS attacks from mobile clients. The natural features of mobile clouds provide good conditions for DDoS attacks. Every mobile operating system has millions of users connected to the Internet, counting on the same cloud server to synchronize their configurations and data files. The synchronization system is usually preconfigured to access the cloud server in different time to ensure the load for the cloud server. If the system is cracked by the attackers, or if the synchronization mechanism is known by them, they can make all the mobile devices start requests together. This will cause a high pressure to the cloud servers and may affect the availability of the service. 


\subsection{Methods of Defense and Remaining Issues}

Since the cloud-specific vulnerabilities and threats have been listed, the methods in recent researches for defending those threats are also illustrated in this chapter. We categorize them according to their corresponding threats.

\subsubsection{Defending DoS Attacks to Cloud Systems}

Multiple ways are presented to help prevent the improper use of cloud computing. Generally they focus on monitoring users environments and behaviors, such as their operating systems and service type they are providing. In addition, researches have been done on measuring the resource usage to judge if users are attacking others or being attacked.

A new architecture was introduced [33] to secure the customers' virtualized workloads in a cloud setting. The discovery and integrity measurement of code and data were combined starting from hardware state. Another method was proposed to detect and avoid DoS attacks in clouds. There was a monitoring agent either in a different subnet or outside the cloud infrastructure. The agent and the application constantly probed each other to see the bandwidth available in both directions. When the application found the bandwidth lower than the threshold, it sent UDP packets to the monitoring agent to ask for help. Then the agent would initiate application migration by launching different instances in different subnets. It also enabled application hopping which moved applications to different servers periodically. The problem of this method is the overheads of the monitoring agent, which also costs considerable resources for multiple applications, especially when the network load is high. Besides, the application hopping is redundant because part of the applications is not the DoS target. Sandhu et al. 34 gave the outline of the research agenda, including developing new protocols to cope with DoS and insider attacks and to ensure predictable 
delivery of mission critical data. Additional routing protocols that enable VM redundancy and VM migration should be developed, as well as peer-to-peer communication mechanisms which hide mission-critical data and VMs. These mechanisms will lead to additional costs on VM migrations and will make the routing more complex when using peer-to-peer technologies.

\subsubsection{Inhibiting Side-channel Attacks}

One way to mitigate or eliminate side-channel attacks is to prevent side-channel vulnerabilities. The usual methods are using the blinding technologies to minimize the information leakage, such as cache wiping, random delay insertion, adjusting each machines perception of time, etc. Lots of countermeasures are presented and discussed to avoid the information leakage by measuring technologies. However, there are two drawbacks in these countermeasures [16. First, they often have a pretty high overhead and therefore impractical. Second, they require unconditional anticipation of side channels which is impossible in state-of-art VM co-residence.

Many other efforts have been made to eliminate side channels. A new approach was proposed for timing channel control [35], using provider-enforced deterministic execution instead of resource partitioning to eliminate timing channels within a shared cloud domain. The basic idea was to make the cloud behave like a batch job processor. The provider broke each job into smaller work units and used load-balancing algorithms controlled by the provider to distribute work among cloud servers, therefore only leaving one controllable timing channel at the boundary. Two resource

management approaches were advocated [36] to provide performance and security isolation in the shared cloud infrastructure: cache hierarchy aware core assignment and page coloring based cache partitioning. However, neither of the approaches completely eliminated side channels because of sharing still existed. Information leakage 
might happen if the deterministic execution method is exposed. Vattikonda et al. considered another approach [37] to avoid the risk of cross-VM side channels, which was weakening malicious virtual machines' ability to receive the signal by eliminating fine-grained timers. They made the timer provided by the RDTSC instruction on Xen-virtualized x86 machines substantially more coarse. On the other hand, it was difficult to determine malicious VMs and such operations may violate the SLA agreements.

There are also researchers trying to find a balance between sharing and security. Keller et al. proposed removing the virtualization layer, while retaining the key features enabled by virtualization [38]. Their architecture called NoHype provided benefits on par with those of today's virtualization solutions without the active virtualization layer. The key aspects included running one VM per core, hardware enforced memory partitioning and dedicated devices without hypervisor. Though it did help protect the information, it was actually a setback from cloud computing to traditional computing. An extreme case is using a dedicated server which does not have side channels at all.

\subsubsection{Ensuring Safe Authentication and Encryption}

Since the vulnerabilities exist in authentication and encryption processes, researches have been done to ensure the integrity of the cloud systems while avoiding data leakage. The solutions include creating verification services, the range of applying cryptography and so on. The purpose of authentication and encryption is to protect multiple interfaces and APIs existing in the cloud systems and sensitive data from the users.

Part of the researchers put emphasis on authentication and authorization solutions. Du et al. present a novel RObust Service Integrity Attestation framework 
in 39 that cloud verify the integrity of state-of-art data flow processing services and pinpoint malicious service providers within a large-scale cloud system. Chow et al. developed a concrete cloud authentication system called TrustCube [40]. With this system, any cloud-based service could redirect authentication to the authentication service via a federated authentication framework such as OpenID. Thus users only needed to trust the authentication service providers and did not have to maintain the authentication service by themselves. But TrustCube are not suitable for services requiring private user accounts and authentications.

Chen et al. explored whether cryptography could be deployed to secure cloud computing against malicious insiders [17]. They estimated common cryptography costs and finally explored outsourcing of data and computation to untrustful clouds. They showed that deploying the cloud as a simple remote encrypted file system was extremely unfeasible if considering only core technology costs. They also concluded that existing secure outsourced data query mechanisms were mostly cost-unfeasible because of limited computation power of today's cloud. Danezis et al. showed that

integrity and privacy could be cryptographically achieved today for a broad class of processing problems on personal data [41]. They argued that cloud computing could be used to leverage and enhance those technologies in terms of availability. Then they presented two examples of real world privacy problems. However, the overhead was still considerable and would cause cost on both performance and budget. This trade-off needs to be discussed further.

\subsubsection{Protecting the Privacy}

When the account or service is inevitably hijacked, we need to minimize the loss or leakage of the data, especially for the sensitive data and files. With the cloud service working together with local services such as private cloud or local stations (traditional 
or mobile), the computation split technology can be used to keep private data away from vulnerable zones.

The authors of [34] argued that the twin issues of availability and security in the cloud could be adequately addressed only if we made transition from current missionoblivious clouds to mission-aware clouds. A system called Sedic [42] was developed which automatically partitioned a computing job according to the security level of the data it worked on, and arrange the computation across a hybrid cloud. In this way, the tasks within the private cloud only worked on sensitive data and those on the public cloud only processed public data. Thus all the non-private workload could be offloaded to the low cost commercial cloud.

The problem of these solutions is that they are lack of theoretic analysis on the optimum split method of computation and the communication and encryption cost by splitting jobs.

\subsubsection{Risk Configuration and Profile Assessment}

Cloud services often ignore reminding users of setting the profiles of configurations correctly. Besides, cloud images have the same problem and also have new risks. Researchers tried to make the configuration process simpler by automating the extraction of configurations, managing the images and detecting the vulnerabilities outside the images, and reminding users of the risks they may take.

Various analysis models have been proposed to assess the vulnerabilities and risks in the cloud. Bugiel et al. focused on the threats caused by unaware users [43]. They considered security and privacy aspects of real-life deployments and gave a detailed systematic analysis of various crucial vulnerabilities in publicly available Amazon Machine Images (AMIs) and showed the way to eliminate them. A quantitative risk and impact assessment framework was presented 44] to assess risks associated 
with cloud computing platforms. The framework defined risk as a combination of the probability of a security threat event and its severity, measured as its impact. QUIRC's key advantage was its fully quantitative and iterative convergence approach, which enabled stakeholders to comparatively assess the relative robustness of different cloud vendor offerings and approaches in a defensible manner. A new approach 45] was presented to assess the end-user configuration of multi-tier architectures deployed on infrastructure clouds. By automating the process of extracting the configuration and proposing a query and policy language, it was available to analyze the reachability and vulnerability of services in the virtual infrastructure. Wei et al. explained the new risks of a cloud's image repository [46]. The authors proposed an image management system which could control access to images, track where the image came from and help users detect and repair security violations.

\subsubsection{Securing Mobile Cloud Computing}

Because of the constrained resources and massive private user data in mobile device related cloud computing, the security of mobile cloud services should be considered and protected in its own way despite the general methods mentioned above.

The design considerations to build secure elastic applications was presented [4], including a solution for authentication and secure session management between weblets running device side and those on the cloud, as well as how to authorize cloud weblets to access sensitive user data. The weblets represented the part which could function individually but can communicate to each other. The elasticity manager decided which part ran on the mobile device and which one ran on clouds. Usually the parts requiring more resources such as video and image processing were assigned to the cloud services while the UI parts and those need local data access were assigned 
to the mobile devices. To make this architecture secure, the integrity of weblets installation was checked first by SHA1 signature verification. Authentication between weblets was built then to ensure secure communications between different weblets by generating session keys and secrets. The migration process was also secured by a serious of operations among mobile devices and cloud mangers, ensuring that all sides would know the result of migration to guarantee service availability. Finally, they use different authorization methods for external cloud services and internal weblets communications.

Liang et al. classified cloud security services in two categories: Critical Security (CS) service and Normal Security (NS) service [48]. CS service provided strong security protection such as using longer key size, strict security access policies, and isolations for protecting data, and so on. The CS service usually occupied more cloud computing resources. However, it generated more rewards to the cloud provider since the CS service users needed to pay more for using the CS service. They proposed a Security Service Admission Model based on Semi-Markov Decision Process to model the system reward for the cloud provider.

A secure mobile user-based data service mechanism was proposed [49] to provide confidentiality and fine-grained access control for data stored in the cloud. The mobile user could securely shift the data computing and distribution overhead to the cloud while the cloud had no idea about data content in the whole process. Additionally only authorized users can decrypt the cipher text while unauthorized users would learn nothing about the data. They put emphasis on identity based proxy re-encryption scheme to let mobile devices easily implement fine-grained access control of data. The data owner only needed to forward a re-encryption key of a small size instead the cipher text to gain the access control to a file. However, the security of data when transmitting still kept the same mechanism as before, which also had a significant 
cost compared to that of access control.

In general, the existing work on securing mobile cloud computing focuses on splitting the software into different modules, protecting communications between mobile devices and cloud servers, and authentications for mobile clients which access data in the cloud. The DDoS attacks from mobile devices as the analysis we did in last chapter should be considered. Besides, the availability of putting software modules into cloud servers may be affected by the communication load due to the variety of network connection quality. So the communications between mobile devices and cloud servers should be reduced as much as possible.

\subsection{Cloud-assisted Mobile Computing and Its Vul- nerabilities}

The powerful computational resources and acceptable prices of cloud computing leverage great opportunities to combine clouds and mobile computing together. A lot of work has been done to take the advantage of cloud computing by offloading jobs and applications from mobile devices to VMs in the cloud. Although it can save limited power of mobile devices and accelerate the speed of executing applications, the security issues cannot be ignored during the fast development of such cloud-assisted mobile computing.

Cloud computing for mobile devices is becoming a more and more important topic since the wide deployment of related applications. Apple's iCloud synchronization system is a good example. It can make backup for everything in the mobile devices to the cloud server, including application profiles, photos, configurations, etc. Backberry messenger and email services from Research In Motion (RIM) also rely on cloud services because the company takes Amazon EC2 as their email and messenger servers. 
The shared technologies used by cloud computing systems are the most significant difference comparing with traditional systems. For different service models, shared technologies lead to different vulnerabilities and threats as illustrated in Figure 2.2. In such a public, insecure computation environment, both users and cloud service providers are not supposed to trust each other.

- User data leakage. The personal data such as photos and configurations from different users is stored together, making it possible for malicious users or attackers to compromise a small part of the cloud and then get a block of data which contains information from many users.

- Mobile user traffic analysis. Mobile devices can use Internet service either from service operators or from WiFi connections. The connection to cloud servers will also work in untrustful data service, which may have the risk to be eavesdropped by attackers. By collecting information from multiple WiFi access points, attackers have the ability to gain the communication mechanism of the synchronization applications with cloud servers. This is helpful for understanding the transmission protocol, blocking the services or causing data burst at the same time for a lot of mobile clients.

- DDoS attacks from mobile clients. The natural features of mobile clouds provide good conditions for DDoS attacks. Every mobile operating system has millions of users connected to the Internet, counting on the same cloud server to synchronize their configurations and data files. The synchronization system is usually preconfigured to access the cloud server in different time to ensure the load for the cloud server. If the system is cracked by the attackers, or if the synchronization mechanism is known by them, they can make all the mobile devices start requests together. This will cause a high pressure to the cloud servers and may affect the availability of the service. 
This report identifies the different relationships between users and service providers that cannot be trusted, and tries to let the clouds and mobile devices cooperate with each other. In order to achieve this objective, we investigate the job partition mechanisms on both conventional distributed systems and mobile cloud systems. The literature survey shows that it is possible to enable offloading jobs into cloud using Java-based at the method level. Then the essential problem becomes how to partition the jobs efficiently and securely. To answer this question, a model has been built, aiming to offload part of the application into the cloud, while guaranteeing the sensitive parts staying locally.

Recent researches focus on the advantage of offloading mobile applications into remote locations, including both cloud servers and ubiquitous computation facilities nearby. Various systems and architectures have been proposed [1 3, 47,50,52]. These systems usually aimed to achieve higher computing ability and lower energy consumption for resource-constrained mobile platforms. Resource-allocation optimization schemes have been presented [48,53 with the purposed of minimizing energy consumption. The security issues have also been discussed [40, 47, 54 to build safer authentication processes or help detect malicious applications.

\subsection{Job Partitioning Between Clouds and Mobile Devices}

This section mainly discusses one of the most substantial problems in architectures that can offload whole or part of applications into the cloud, that is, which application, or which parts of one application, should be offloaded into the cloud. The problem can be summarized as the optimal partition problem to achieve minimum cost for both mobile devices and cloud services. Several research groups have built models for 
mobile application execution and have done analysis based on the models. The rest of this section introduces types and related models to partition the applications and modules, and then to offload proper parts into the clouds.

\subsubsection{Application-level Partitioning Based on VM Images}

Application level partitioning means to take the application as the offloading unit, that is, to offload the whole application into the cloud. This kind of partitioning and offloading is usually based on VM images in the cloud. Each user has a remote VM image which has the similar configurations to the real OS running on the mobile device. As a result, most applications will run smoothly in the VM after they have been offloaded. The Cloudlets [1] and CloneCloud [2, 3, 50] systems are introduced, both introducing VM images in the cloud.

Satyanarayanan et al. present VM-based Cloudlets in mobile computing [1]. The authors argue that distant cloud services are not capable for those mobile applications sensitive to delay. Instead, they address mobile device's resource poverty via a nearby resource-rich cloudlet. Each cloudlet has the ability to create universal base VMs for mobile devices. And mobile devices will transmit the VM overlay to the cloudlet when they need to launch VMs. With the help of cloudlets nearby, users can avoid WAN delays while benefiting from the rich resources of cloudlets.

The concept of mobile application offloading is adopted to save limited energy of mobile devices. A group from Intel Research Berkeley conducts a series of researches on the combination of smartphones and cloud computing [2,3,50]. They propose an architecture [2] that seamlessly offloads execution from the smartphone to a cloud infrastructure with smartphone clones.

Paranoid Android [54 uses QEMU to run replica Android images in the cloud to enable multiple exploit and attack detection techniques to run simultaneously with 
minimal impact on phone performance and battery life.

\subsubsection{Module level partitioning}

The module level partitioning dates back to over 10 years ago, when it was initially used for deploying distributed computing framework with multiple servers. The most representative schemes include Coign [55] in Microsoft Windows and J-Orchestra [56] based on Java RMI [57]. J-Orchestra [56] is also an automatic partitioning system. It is exclusively designed for Java platforms. It provides the solution for distributed computing for Java programs. It operates at the Java byte-code level and rewrites Java application byte-code and replace local data exchange with remote communication. ThinkAir [4] provides an efficient way to perform on-demand resource allocation, and exploited parallelism by dynamically creating, resuming, and destroying VMs in the cloud when needed.

\section{Weblets}

Building elastic applications in mobile systems is considered in 477 to save limited energy of mobile devices. An elastic application consists of multiple weblets. They can be launched on both mobile devices and clouds. A device elasticity manager is launched on the mobile device to make runtime configuration changes, such as the location of running a weblet (local or remote), the way of collaboration between mobile devices and the cloud, and how to communicate with the cloud (WiFi or $3 \mathrm{G})$. There is a cost model responsible for making configuration decisions, which accounts for several factors like power cost of the device and the monetary costs of the network and cloud usage. Meanwhile, the cloud elastic service is in charge of resource management in the cloud, with a cloud manager, an application manager and a cloud node manager included. It provides a web service interface and can be 
launched on multiple cloud nodes.

\subsection{Scheduling and Optimization in Job Partition- ing}

It has been proved feasible in Section 2.5 that the jobs in an operating system can be partitioned either by moving applications to VM replicas or offloading the methods. The efficiency of the partition is decided by which jobs are moved into the remote

cloud. Specifically, it is related to the CPU and communication cost and the delay constraints, and so on. In this section, several related researches for modeling and optimizing the application partition are introduced and compared.

\subsection{Securing Cloud-assisted Mobile Computing}

The security-aware cloud-assisted mobile computing frameworks are introduced in this section. They are related to authentication [40,47], malicious application checks [54], and resource optimization [48].

The elastic application architecture [4] also addresses the sucurity issues that it may cause threats from mobile devices, cloud servers and communication channels. To secure the elastic applications, the system used SHA1 signature for integrity verification during the application installation. The authentication between weblets is also introduced to ensure weblets collaborate safely in different locations. Then the safe migration process is discussed for moving weblets to new locations when needed. However, only general architecture is presented in this work without convincing numerical or experimental information such as how to implement the cost model, the efficiency and latency of cooperating with remote clouds, and what the exact overhead 
is securing the elastic applications.

A flexible framework called TrustCube is presented [40] supporting implicit authentication (IA) which translates user behavior into authentication scores. The authors focuse on mobile data and built and IA system based on calling patterns, SMS activity, website accesses, and locations. The TrustCube system resides in the cloud and provided IA service. The architecture is divided into four parts: client devices, data aggregators, an authentication engine and authentication consumers. Implicit information is collected by the client devices and was sent to data aggregators. The latter collects information from both client devices and third parties, and helps the authentication engine make decisions to respond requests from client applications. According to the authentication result, the applications will either return the desired contents or reject the request. Although the framework could reduce the possibility of adversary usage of mobile devices, the potential violation of user privacy for investigating their phone calls, SMS, and so on, is itself a vulnerability. Besides, the article did not mention the cost for synchronizing implicit information.

Paranoid Android [54 uses QEMU to run replica Android images in the cloud to enable multiple exploit and attack detection techniques to run simultaneously with minimal impact on phone performance and battery life. They perform security checks in clouds by recording and transmitting a minimal trace of the phone's execution for replaying on the cloud server. This method is good for detecting malicious applications at a low cost. However, it would not help save energy or resource for mobile devices. It might also be too late for the replay-and-detect mechanism to stop malicious applications from leaking private information. 


\subsection{NFV Placement, Resource Sharing and Blockchain}

The ETSI architecture of MEC [58] leverages the existing NFV framework [59] to achieve dynamic and fast MEC service provisioning. To our best knowledge, there has not been much research on mobile edge application placement. Main references of related work are still from the NFV world.

NFV faces new research challenges due to the new features it introduces, such as flexibility of deploying network functions and similar pricing model to cloud computing [60]. Among many other research challenges, mobile edge application placement policies have been studied with various focuses and is typically modeled as a resource allocation problem. Deployed without consideration of optimal resource allocation, real-world mobile edge application deployments are found inefficient to utilize resources through instrumentation effort [61]. The goal of the resource optimization is to find on the best physical resources (servers) to place network functions. Such problems are formulated and studied in 62].

For the VM placement issue in a cloud data center, a comprehensive study of the VM placement and consolidation techniques used in cloud was presented in 63. The VM placement problem and various approaches were reviewed. The placement techniques were classified as constraint programming, bin packing, stochastic integer programming and genetic algorithm. Research work in [64 and [65] focused on VM allocation in data centers as well. With an optimal technique, 64 aimed to minimize the number of required VMs, with considerations of each VM's resource limitation. A VM placement algorithm was proposed in [65 with the objective to minimize carbon footprint. An NFV traffic steering problem was discussed in [66], where the limited resources were taken as constraints to determine the lowest cost and to steer traffic 
accordingly.

In mobile edge application networks, the virtual resources include virtual machines, which execute either virtual routers or virtual service elements. To address the challenges related to the management and orchestration of virtual resources, Clayman et al. 67] described an architecture based on an orchestrator that enabled automated placement of virtual network and processing resources across the physical resources of the network. Least used placement, N-at-a-time placement and Least Busy placement algorithms were presented. Similar to [67], [68 focused on the mobile edge application deployments by presenting a model for resource allocation in NFV networks, while also considering the difference between service requests and VM requests. It addressed the scenario where part of the services may be provided by dedicated physical hardware, with the other part using virtualized service instances. A basic model using linear programing was defined for NFV resource allocation, with the constraints of server capacity and the number of instances on a node. The objective of the model is to minimize the number of user servers. To add network-awareness to the model, constraints related to the request flow were added.

Service function chaining (SFC) has been used widely in carrier networks [69], in order to allow configuring network services dynamically without having to change networks at the hardware level. A service graph is used to describe service chains and traffic is routed according to the graph. Use cases leveraging SFC include network functions for packet inspection, traffic optimization, protocol proxies, and value-added services. Network service headers were used $[70]$ to provide data-plane information for constructing topological-independent services. A software-defined architecture to enable SFC was proposed in 71 leveraging OpenFlow with discussion of functionalities, challenges, testbeds, and other aspects implementation-wise. A heuristic resource allocation algorithm was proposed for VNF chains called CoordVNF [72]. Substrate 
nodes with enough resource to deploy VNF were explored and the subsequent nodes were checked iteratively. If any of the substrate nodes cannot embed a VNF in the chain, backtracking was performed.

When attempting to achieve various goals, existing work does not appear to consider the combination of low cost and high availability together with practical placement policies, which are affinity and anti-affinity, In this dissertation, these factors are weighed in, and the strategy is ready for use by real-world mobile edge application scenarios.

By leveraging generic cloud computing IaaS frameworks, such as OpenStack 73 and VMWare [74], research on cloud-based NFV has been done to ensure that VNFs run at optimum levels in the cloud [60]. Soares et al. presented a platform for VNFs called Cloud4NFV [13], which is compliant with the ETSI [75] NFV architectural specification. Two approaches were discussed to virtualize NF: full virtualization moved all control and user plane functional entities to the cloud, while partial virtualization still forwarded user traffic to physical hardware. With the implementation of service provisioning and end-to-end inventory management, vConductor [76] was presented by Shen et al. that enabled users to plan the virtual network services using its data model. The systems and architectures above focus on deploying VNF instances into the generic cloud infrastructure, rather than the edge of the network.

Due to the nature of varying cost of resources in the cloud, cloud-based resource allocation problems have been studied to reduce the cost and to help evenly distribute the workload. We have analyzed vulnerability of mobile apps in [77] to keep sensitive information in local mobile device, while offloading secured computing-intensive modules to the cloud. Xiao et al. [52] presented a system leveraging virtualization to dynamically allocate resources in datacenter and to optimize the number of servers in use. While these solutions did help better use cloud resources, they keep the 
computing remotely in the cloud and will not move it to the edge of the network.

The concept of fog computing was proposed in [24] and was anticipated to become an essential part of cloud computing with the number of Internet-of-Things (IoT) growing explosively. Vaquero et al. 78 proposed a comprehensive definition of the fog covering its features and impact, including device ubiquity, challenges on service and fog-based network management, levels of device connectivity, and privacy. Edge clouds were presented as entry points for IoT, which could be parts of the Enhanced Packet Core (EPC). The scenarios of fog computing in several domains were discussed in [79], including Smart Grid, IoT and SDN, with topics about security, privacy, trust and service migration. The work above has pointed the research direction of leveraging the edge of the network from high levels. Based on fog computing, crowdsourcing becomes an option as fog nodes can be updated dynamically with the participation of the third party. The security and privacy challenges were illustrated in [80], where a general architecture was presented to model crowdsourcing networks, including crowdsourcing sensing and crowdsourcing computing. The security concerns were captured from the characteristics of the architecture.

Virtualization in edge networks as a form of fog computing, including NFV, have been given a close look. Manzalini et al. visioned potential value chain shifts and business opportunities in [81] by emerging paradigms such as SDN and NFV. The paper pictured a massive number of virtualized network and service functions running at the edge of the network, making the processing power more distributed globally. The service chaining in the cloud-based edge networks was analyzed in [82] by programming actions into OpenFlow switches to achieve dynamic service chaining. A platform called Network Functions At The Edge (NetFATE) was proposed in 83 as a proof of concept (PoC) of an NFV framework at the edge of a telco operator networks. Each CPE node was realized with a generic-purpose computer installed 
with a hypervisor and virtual switches. This made the CPE node capable of deploying VNFs on itself. The focus of this paper was to prove that deploying VNFs on the edge of the network is feasible. However, the benefits of resource sharing across different CPE nodes are not mentioned.

\subsubsection{Blockchain and Resource Allocation}

The research directions in network service chaining (NSC) were discussed in [84]. For security considerations, the authors highlighted the difficulty of bringing shortlived network services to targeted users in a single subscriber network by using the current security schemes. The potential security problems in SFC were stated in RFC7498 [85], including service overlay security, trusted classification policy, and secure SFC encapsulation. We investigated a placement problem in MEC with the consideration of application availability in [86].

Xiong et al. proposed a pricing strategy for offloading the blockchain's resourceconsuming proof-of-work tasks to edge computing nodes [87]. A two-stage Stackelberg game model was presented with both the edge computing service provider and the miners involved. A hierarchical distributed control system was built using Hyperledger Fabric blockchain [88]. The hosting locations of cloud and fog of blockchain were compared in 89] for IoT networks with the conclusion that fog nodes were better as network latency was the dominant factor.

Nakamoto introduced the concept of blockchain and implemented Bitcoin [90], a decentralized cryptocurrency that first resolved the double spending problem. Blockchains are based on Merkle trees 91] to efficiently allow multiple documents to be saved together in a block. As a decentralized public ledger, blockchains can serve beyond cryptocurrencies. Ethereum [92 used blockchain to store smart contracts that support building virtually any decentralized application. 


\section{Chapter 3}

\section{Vulnerability For Offloaded Applications}

\subsection{Introduction}

Cloud computing provides computational resources with high availability and reasonable prices. State-of-the-art mobile apps, such as facial recognition, image and video processing, have increased the consumption of on-demand computational resources. Therefore, offloading apps to have them run on cloud servers has emerged [1 4$]$ as an attractive solution in order to exploit the capacity and efficiency of cloud computing. Offloading applications into the cloud would also save limited power of mobile devices and accelerate the speed of executing applications.

One critical issue to resolve in offloading apps is how to manage the security risks of cloud computing 15, 16. Many mobile apps use and store personal information related to banking, health, business, messaging and so on. Sensitive information sent to the remote cloud has a good chance to be exposed to either service providers or malicious customers who have access to the same hardware [16].

Traditional ways to protect remote execution and data storage include mutual authentication, authorization and data encryption for the whole app. If every single part of the app was armored by well-prepared security protections, the system would 
be safe enough but the cost would also rocket up and not be acceptable for commercial use, because too much information has to be encrypted unnecessarily [17]. The redundant encryption and decryption operations hurt both energy efficiency and user experience. We need to consider the trade-offs between security and usability, and to maximize the system security subject to a tolerable delay and resource cost.

Recent research focuses on the advantages of offloading mobile applications into remote locations, including both cloud servers and ubiquitous computation facilities nearby. Various systems and architectures have been proposed [1 4, 47, 50, 51], usually aiming at higher computing power and lower energy consumption for resourceconstrained mobile platforms. Resource allocation optimization schemes have been presented [48,53] with the purpose of minimizing energy consumption. However, there is little discussion about the security of offloading, and mature solutions towards secure offloading cannot be found. In this chapter, we present a mechanism to offload less vulnerable app parts. This approach faces some challenges. First, the complex call relationships of classes and functions within an app make it difficult to highlight the vulnerable parts. Second, it is tricky to determine the level of protection for the parts because of the tradeoffs between security and performance. Besides, no mature systems have been built to break down and offload apps. To address these challenges, we present the following contributions:

(i) We propose a novel graph-based analytical model, namely object dependency graph (ODG). Rather than focus on the whole app as in existing work, we divide an app into multiple parts according to the instances of classes created at runtime, and connect them by their dependencies. The vulnerability of each part may propagate along its dependencies and can be evaluated by our model. The ODG structure enables us to identify problematic objects due to dependencies. 
(ii) We determine the vulnerability level of all app parts and make offloading decisions jointly so that cloud resources can be best utilized, while the security level is not violated. To our best knowledge, we are the first to take this approach that evaluates the propagation of the vulnerability caused by dependencies of objects.

(iii) We design an algorithm based on the ODG model that can run on all popular smartphone OSs. Experimental results based on Android OS are illustrated. The unique point of our solution is the configurable location where the analysis is made. Our analytical algorithms can run either on local devices or in the cloud with the necessary inputs.

\subsection{System Architecture}

Our system is designed to address the security issues arising when mobile devices use public cloud computing services. These issues are mostly caused by the multi-tenancy feature [93] in cloud computing.

\subsubsection{Basic Idea}

The basic idea of the system is to divide an app into components and to keep the components that have the biggest impact on the vulnerability of the app to a local mobile device. Our idea is novel since existing research work on offloading components of apps mostly focuses on energy efficiency rather than on security. A key factor of our approach is the concept of vulnerabilities of an app and its components.

Defining the vulnerability is a challenging task because it refers to risks that may happen. Suppose a large number of copies of an app have been sold. We consider the ratio of the number of compromised instances of a component in the app to the 


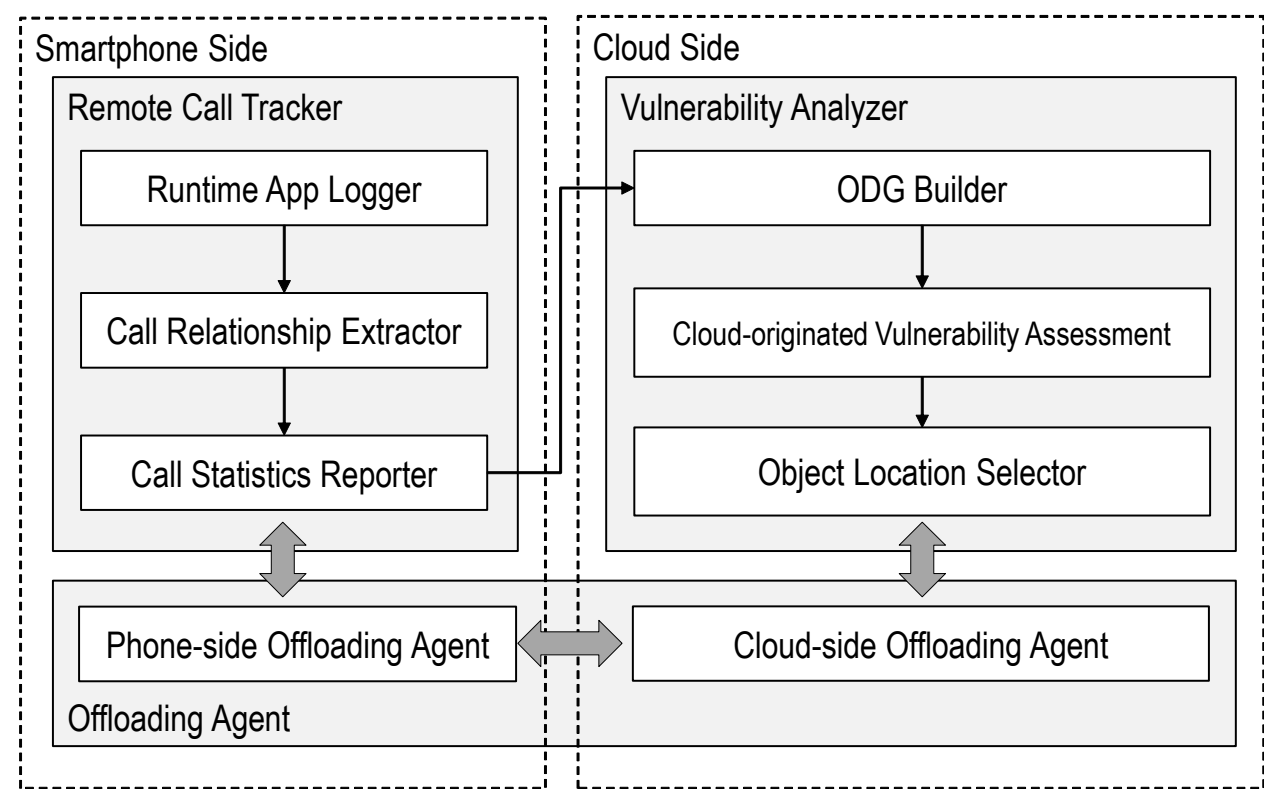

Figure 3.1: System architecture of the vulnerability-based offloading framework

number of instances of the component in use. In theory, this ratio will converge to a probability when the total number of copies of the app in use goes to infinity. The higher this probability is, the more vulnerable the component will be. We define this probability as the vulnerability of the object.

\subsubsection{System Modules}

To realize this basic idea, the system solution needs three functions. The first one is to partition an app into multiple parts. The second is to quantify how vulnerable each part is to ensure offloading the right ones. Therefore, it is paramount to build an analytical model for calculating vulnerabilities of app parts and their impacts on the overall vulnerability of the app for making our offloading decisions. Finally, the system must be able to actually carry out the decisions by offloading the appropriate objects to cloud servers. Meanwhile, we also need to consider the cost of data communications between local and offloaded objects. 

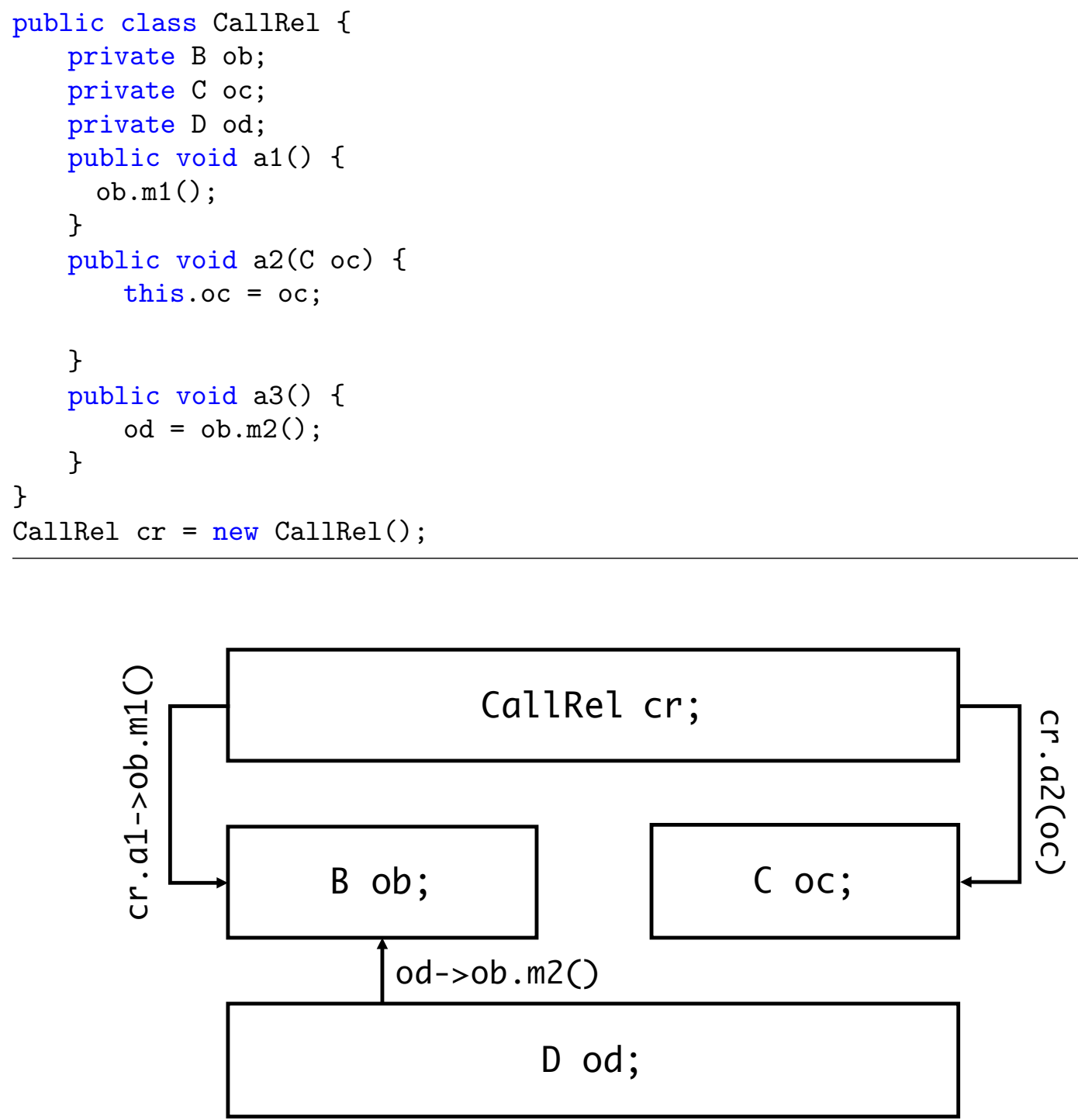

Figure 3.2: Three types of call relationships

Corresponding to the three functions are the three modules in our system as shown in Figure 3.1. The remote call tracker is to properly divide the app into multiple parts to be ready for being evaluated and offloaded. The vulnerability analyzer is to quantify the vulnerabilities and to make optimal offloading decisions. It takes method call statistics from the smartphones as input, and outputs the offloading decisions. The offloading agent is the actual module to fulfill the offloading jobs. 


\section{$3.3 \quad$ Object Vulnerability}

In object-oriented programming (OOP), objects are basic units of a running app as they are instantiated from encapsulated class definitions [94]. An object will be identified as a local object when it runs on the smartphone. And it will be called a remote object if it runs in the cloud.

\subsubsection{Call Relationship and Message Passing}

A call relationship refers to any method invocation or remote message passing that creates dependencies between two objects. The objects depend on each other according to how they are functionally related. On the smartphone side, the method calls defined in classes reveal the dependencies among objects. The forms of method calls are listed below.

- Method Calls: a method in an object calls a method in another object. For example, Object cr in Figure 3.2 has a call relationship with ob, because Method cr.a1() invokes ob.m1().

- Method Parameters: a method in an object has another objects reference as its parameter. Refer to Figure 3.2. Object cr has a call relationship with oc, because oc is a parameter of method cr.a2().

- Method Returns: the value of an object is set by a method call of another object. In Figure 3.2, Object od has a call relationship with ob, because its value is set by the return of ob.m2().

When the app is partly offloaded and the objects are at different locations, the

call relationships would be in form of remote message passing. Depending on the locations and types of objects, there are two types of remote message passing: 


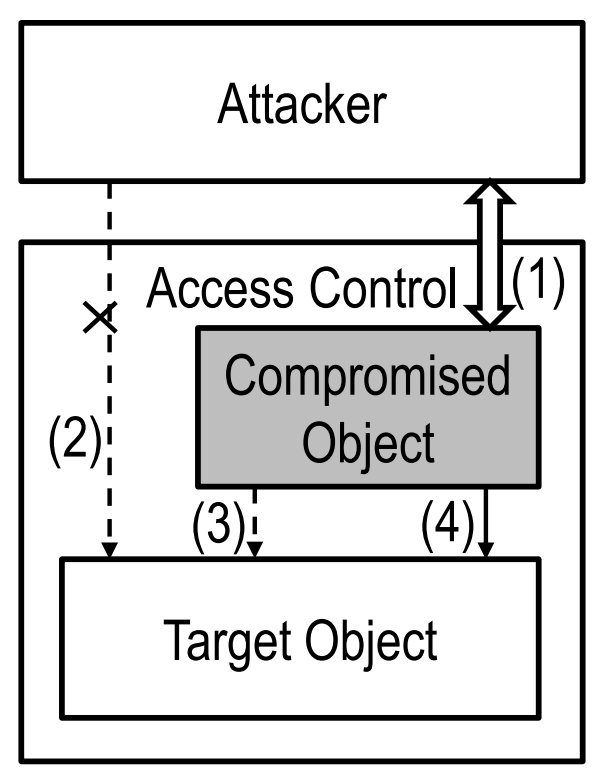

Initiator Attack

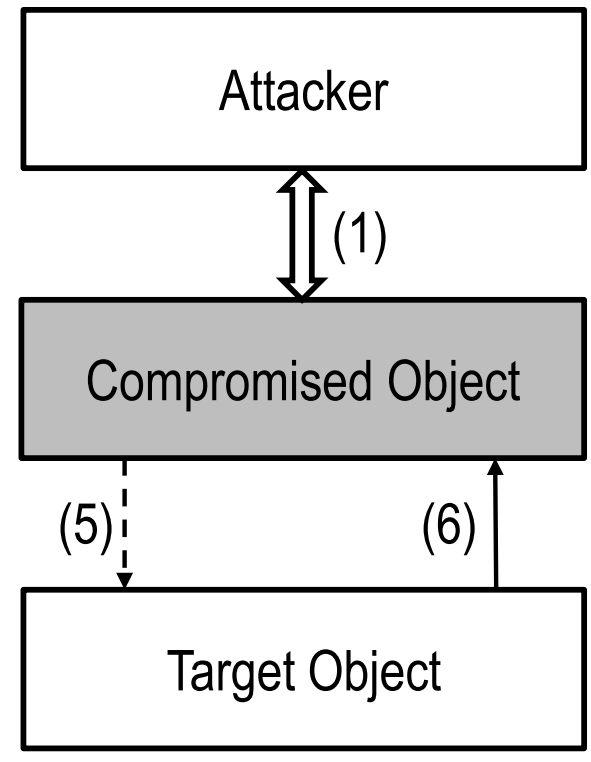

Observer Attack

Figure 3.3: Initiator attacks and observer attacks

- Local-remote Message is the message passed between a local and a remote object. Smartphones communicate with cloud services through local-remote messages. And the propagation of vulnerabilities by these messages will affect the information on smartphones directly.

- Remote-remote Message stands for the message passed between two objects in the cloud. The remote-remote messages do not interact with the smartphone directly, but they may propagate vulnerabilities as well and threat the information security indirectly.

Both method calls and remote message passing enable attacks to gain access to other objects by exchanging data, which lead to the propagation of vulnerabilities, also known as propagated vulnerabilities. We start the analysis from the call relationships, and then define the propagated and the cloud-originated vulnerability. 


\subsubsection{Propagated Vulnerabilities}

The propagated vulnerability of an object is defined as the probability that an attack originates from any other object and propagates to the object through call relationships or message passing. The propagated vulnerability could come from either direction of the call relationship by two types of attacks below:

- Initiator Attacks enable an attacker to take a compromised object of an app as a proxy. The private resources of the app can be reached through the proxy object on behalf of the attacker. The vulnerability in this case propagates from the object initiating method calls or message passing to the target object being called or passed to. Back to the example in Figure 3.2, if ob has been attacked, it may behave incorrectly, such as missing necessary functions and performing malicious operations. Those behaviors also affect Function cr.a1() and then Object $c r$.

- Observer Attacks work in a different way compared to initiator attacks. When a compromised object is called, other objects calling it may pass parameters to it. Those parameters may contain sensitive information. Thus the vulnerabilities can be propagated from the target object being called to the one calling it. In Figure 3.2, if cr has been compromised, attackers may get sensitive information by accessing ob. Similar scenarios happen with message passing too.

The processes of both types of attacks are also described in Figure 3.3 , For both attacks, the attacker has the control of a compromised object shown by Figure 3.3(1). For initiator attacks, the attacker cannot compromise the target object directly along Figure $3.3(2)$, as there are access control mechanisms to protect illegal 
accesses. However, attackers may take the compromised object as a proxy, and propagate the vulnerabilities to the target object as illustrated by Figure 3.3(3) through the call relationship of Figure $3.3(4)$. The direction of the vulnerability propagation and that of the call relationship are the same. For observer attacks, if the target object calls the compromised object through the call relationship of Figure $3.3(6)$, then the compromised object may send a forged response that could compromise the target object. So the vulnerabilities propagate from the compromised object to the target object, whose direction is opposite to the call relationship.

The results of vulnerability propagation along opposite directions of call relationships are equal: whichever attacks propagate, the fact that both objects from such call relationship or message passing become compromised remains the same. Thus the ODG can be modeled as an undirected graph.

\subsubsection{Cloud-originated Vulnerabilities}

The cloud-originated vulnerability is caused by interactions between an object and the cloud environment hosting it. It is defined as the probability that an object is compromised due to the weaknesses or the attacks from the cloud environment. Specifically, it may be caused by memory and CPU cache leaks [95], or side/covert channels in the cloud [16]. Because it may be very hard to identify the exact sources that induce the cloud-originated vulnerability, we consider the compromised object as the originator if the vulnerability is caused by the cloud environment.

A feasible way to evaluate cloud-originated vulnerabilities is through measurement. To this end, the reputation-based systems can be adopted [93]. Being widely used in mobile Ad-Hoc Networks [96], E-mail Anti-spam 97], online shopping [98 and social networks [99], reputation systems provide us practical solutions to estimate cloud-originated vulnerabilities. It is possible to estimate the cloud-originated 
vulnerability of a component based on cross-analysis of the vulnerabilities of multiple apps if they share one or more components.

The research on the cloud-originated vulnerabilities will be out of the scope of this dissertation. Instead, we will focus on the propagated vulnerability as it is less studied. We will take the cloud-originated vulnerability of each object as a known variable.

\subsubsection{Object Vulnerabilities}

The vulnerability of an object can be defined now as the probability of that object to be compromised due to either cloud-originated or propagated vulnerability. The mathematical descriptions of the definitions above will be modeled in the next section.

\subsection{Object Dependency Graph}

The definitions of symbols used below can be found in List of Symbols. We model all objects in an app as a set of nodes $V$, and all their call relationships as a set of undirected edges $E$. They form an undirected graph $G=(V, E)$ for the app, namely Object Dependency Graph (ODG). The ODG of an app reflects the dependencies of objects propagating vulnerabilities. In the following sections, we will use either "objects" or "nodes" for objects in ODGs. We will also use "call relationships" or "edges" for dependencies of objects.

We begin the modeling of the ODG with $A_{o o}$ to denote the random variable of the originator o being compromised for its cloud-originated vulnerability, and $p_{o o}$ as the probability of $A_{o o}$ to occur, also known as the cloud-originated vulnerability: $p_{o o}=P\left\{A_{o o}\right\}$. Let $m$ denote the index of the path the attack takes from $o$ to the target object $d$, and let $r_{o d}^{m}$ denote that path. Let $A_{o d}^{m}$ be the random variable that $o$ 
has incurred a cloud-originated attack and the attack has propagated to $d$ along $r_{o d}^{m}$ such that

$$
A_{o d}^{m}= \begin{cases}1, & \text { if an attack propagates on the path } r_{o d}^{m} \\ 0, & \text { if no propagation found on the path } r_{o d}^{m}\end{cases}
$$

Let $p_{o d}^{m}$ denote the probability of $A_{o d}^{m}$ to occur. For $r_{o d}^{m}, A_{o d}^{m}$, and $p_{o d}^{m}, m$ can be removed if there is only one path from $o$ to $d$. Then they are denoted by $r_{o d}, A_{o d}$, and $p_{\text {od }}$. Also let $e$ denote an edge in the ODG and $p_{e}$ be its propagated vulnerability. We suppose the vulnerability propagation is only related to the features of the edge itself. Then the event of the vulnerability propagation along each edge is independent. Hence we have the propagated vulnerability along $m$. The propagated vulnerabilities need to be calculated based on observed vulnerabilities and the topology of the ODG. The calculation method will be shown in the following sections. The results can be reused if duplicated objects are found.

$$
\begin{aligned}
p_{o d}^{m} & =P\left\{A_{o d}^{m}\right\} \\
& =P\left\{A_{o d}^{m} \mid A_{o o}\right\} P\left\{A_{o o}\right\} \\
& =p_{o o} \prod_{e \in r_{o d}^{m}} p_{e} .
\end{aligned}
$$

Given Equation 3.2 above, and let $E_{d}$ denote the randome variable that $d$ is compromised for any reason, so that $P\left\{E_{d}\right\}$ can denote the object vulnerability of $d$. The propagated vulnerability of $d$, denoted by $\mu_{d}$, is the probability that an attack originates from any object $o \in \mathbb{V}-d$ and then propagates to $d$ :

$$
\mu_{d}=P\left\{E_{d} \mid \overline{A_{d d}}\right\}=P\left\{\bigcup_{o \in \mathbb{V}-d} A_{o d}\right\}
$$


The object vulnerability is denoted by $\pi_{d}$, where

$$
\pi_{d}=P\left\{E_{d}\right\}=P\left\{\bigcup_{o \in \mathbb{V}} A_{o d}\right\}
$$

\subsubsection{Vulnerability Propagation Model in ODGs}

In this section, an analytical model is to be built to evaluate the vulnerability of an arbitrary vertex in a general ODG. Before we start, without loss of generality, the following assumptions are made for calculation simplicity:

- Each object $o \in \mathbb{V}$ has the same cloud-originated vulnerability $p_{o o}=\alpha$;

- Each relationship $e \in \mathbb{E}$ has the same probability $p_{e}=\beta$ to propagate an attack.

Refer to the method in [100] that was used in reliability theory, we define the two sets below:

- Minimal path sets, denoted by $R_{o d}=\left(r_{o d}^{1}, r_{o d}^{2}, \cdots, r_{o d}^{M_{o d}}\right)$, is the set of all minimal paths. A minimal path is a set of edges that comprise a path, but the removal of any one edge will cause the resulting set not to be a path. In other words, if all the edges in a minimal path are compromised while all other edges are working properly, the target object will be compromised. If any one of the edges in the minimal path subsequently is not compromised, the target object will not be compromised because of this path. Figure 3.4 shows an example of the minimal path set. As an example of the minimal path sets, it has four minimal paths: $R_{o d}=\left(r_{o d}^{1}, r_{o d}^{2}, r_{o d}^{3}, r_{o d}^{4}\right)$, distinguished by different line dashes. If consider the graph as an example of the minimal cut sets, there are 4 minimal cuts in gray lines: $C_{o d}=\left(c_{o d}^{1}, c_{o d}^{2}, c_{o d}^{3}, c_{o d}^{4}\right)$. The four minimal paths are illustrated by different line dashes. The minimal path sets can be found via a depth-first search (DFS) that traverses all minimal paths. 
- Minimal cut sets, denoted by $C_{o d}=\left(C_{o d}^{1}, C_{o d}^{2}, \cdots, C_{o d}^{D_{o d}}\right)$, is the minimal sets of edges whose failure of vulnerability propagation ensure the failure of the attack propagating from $o$ to $d$. $D_{o d}$ is defined as the number of minimal cut sets. Again, Figure 3.4 shows an example of the minimal cut set. The minimal cut sets can be found via the CARA algorithm 101 originally used in the fault tree.

According to Equation 3.1 and Equation 3.2, the probability of the vulnerability propagation from $o$ to $d$ along a specific path $m$ can be given by the function below:

$$
p_{o d}^{m}=P\left\{A_{o o}\right\} P\left\{A_{o d}^{m}=1\right\}
$$

Since $A_{o d}^{m}$ is a Bernoulli random variable, we may also compute $p_{o d}^{m}$ by taking its expectation. That is,

$$
p_{o d}^{m}=P\left\{A_{o o}\right\} E\left[A_{o d}^{m}\right]
$$

Suppose there are $M_{o d}$ minimal paths between $o$ and $d$, then we have $0<m \leq M_{o d}$. Let $p_{o d}$ be the probability that $o$ is compromised and the vulnerability is propagated by any minimal path from $o$ to $d$. Then there is

$$
p_{o d}=P\left\{A_{o o}\right\} E\left[\bigcup_{m=1}^{M_{o d}} A_{o d}^{m}\right]
$$

Now we use an example to show the calculation of propagated vulnerability. An ODG with 4 minimal paths from $o$ to $d$ is shown in Figure 3.4. We calculate $p_{\text {od }}$ below for 
this ODG:

$$
\begin{aligned}
p_{o d}= & P\left\{A_{o o}\right\}\left\{1-E\left[\left(1-A_{o x} A_{x d}\right)\right.\right. \\
& \left.\left.\left(1-A_{o y} A_{y d}\right)\left(1-A_{o x} A_{x y} A_{y d}\right)\left(1-A_{o y} A_{x y} A_{x} d\right)\right]\right\}
\end{aligned}
$$

Based on the fact that all events are Bernoulli random variables, we have $A_{y d}^{2}=$ $A_{y d}$. Replacing the propagated vulnerability of each edge with $\alpha$ and $\beta$, we have

$$
\begin{aligned}
p_{o d}= & P\left\{A_{o o}\right\}\left(E\left[A_{o x} A_{x d} A_{o y} A_{y d} A_{x y}\right]-E\left[A_{o x} A_{x d} A_{y d} A_{x y}\right]\right. \\
& +E\left[A_{o x} A_{x d} A_{o y} A_{y d}\right]+E\left[A_{o x} A_{x d}\right]-E\left[A_{o x} A_{o y} A_{y d} A_{x y}\right] \\
& \left.+E\left[A_{o x} A_{y d} A_{x y}\right]+E\left[A_{o y} A_{y d}\right]\right) \\
= & P\left\{A_{o o}\right\}\left(E\left[A_{o x}\right] E\left[A_{x d}\right] E\left[A_{o y}\right] E\left[A_{y d}\right] E\left[A_{x y}\right]-E\left[A_{o x}\right] E\left[A_{x d}\right] E\left[A_{y d}\right] E\left[A_{x y}\right]\right. \\
& +E\left[A_{o x}\right] E\left[A_{x d}\right] E\left[A_{o y}\right] E\left[A_{y d}\right]+E\left[A_{o x}\right] E\left[A_{x d}\right] \\
& \left.-E\left[A_{o x}\right] E\left[A_{o y}\right] E\left[A_{y d}\right] E\left[A_{x y}\right]+E\left[A_{o x}\right] E\left[A_{y d}\right] E\left[A_{x y}\right]+E\left[A_{o y}\right] E\left[A_{y d}\right]\right) \\
= & \alpha\left(\beta^{5}-\beta^{4}+\beta^{4}+\beta^{2}-\beta^{4}+\beta^{3}+\beta^{2}\right) \\
= & 2 \alpha \beta^{2}+\alpha \beta^{3}-\alpha \beta^{4}+\alpha \beta^{5}
\end{aligned}
$$

As we can see above, the calculation of the propagated vulnerability is tedious especially when more paths exist between two objects. It would be useful if we obtain the bounds of propagated vulnerabilities instead.

Recall the definition of $p_{e}$ in Equation 3.2 . Let $i$ be the index of the current minimal cut set in $C_{o d}$. Let $j$ be the index of the current minimal path set in $R_{o} d$. Let $e$ be an edge in the current minimal cut set $C_{o d}^{i}$ or minimal path set $R_{o d}^{j}$.

With $C_{o d}$ we can infer that any successful minimal cut set shall block the propagation of vulnerabilities. So the fact that all minimal cut sets have failed would guarantee a successful attack. Therefore, $p_{\text {od }}$ should be greater than or equal to the probability that no minimum cut set in $C_{o d}$ is satisfied. For any minimal cut set $C_{o d}^{i}$, 


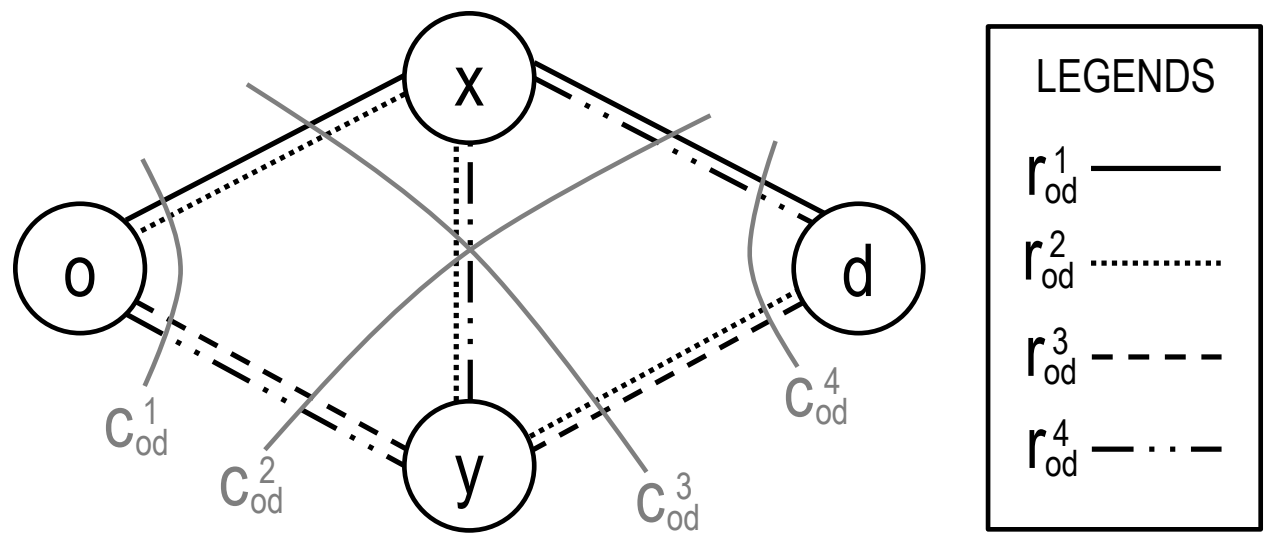

Figure 3.4: An ODG with $M_{o d}=4$

the probability of the event that all of the edges in $C_{o d}^{i}$ have failed to propagate the vulnerability is $\prod_{e \in C_{o d}^{i}}\left(1-p_{e}\right)$. Then $\left[1-\prod_{e \in C_{o d}^{i}}\left(1-p_{e}\right)\right]$ is the probability that at least one edge in $C_{o d}^{i}$ can propagate the vulnerability. Define the events $U_{o d}^{1}, U_{o d}^{2}, \cdots$, $U_{o d}^{D_{o d}}$ by

$U_{o d}^{i}=\left\{\right.$ at least one edge in $C_{o d}^{i}$ can propagate $\}$

The probability of $U_{o d}^{i}$ is then

$$
P\left\{U_{o d}^{i}\right\}=1-\prod_{e \in C_{o d}^{i}}\left(1-p_{e}\right)
$$

Since the vulnerability will propagate if and only if all of the events $U_{o d}^{i}$ occur, we have

$$
\begin{aligned}
p_{o d} & =P\left\{U_{o d}^{1} U_{o d}^{2} \cdots U_{o d}^{D_{o d}}\right\} \\
& =P\left\{U_{o d}^{1}\right\} P\left\{U_{o d}^{2} \mid U_{o d}^{1}\right\} \cdots P\left\{U_{o d}^{D_{o d}} \mid U_{o d}^{1} \cdots U_{o d}^{D_{o d}-1}\right\} \\
& \geq \prod_{i=1}^{D_{o d}} P\left\{U_{o d}^{i}\right\}
\end{aligned}
$$


The last inequality above indicates the lower bound of $p_{\text {od }}$. Replace $P\left\{U_{o d}^{i}\right\}$ according to Equation 3.11, we have

$$
p_{o d} \geq \prod_{i=1}^{D_{o d}}\left[1-\prod_{e \in C_{o d}^{i}}\left(1-p_{e}\right)\right]
$$

On the other hand, with $R_{o d}$ we can infer that at least one minimal path set in $R_{o d}$ should be satisfied to ensure the propagation. For any minimal path set $R_{o d}^{j}$, the probability of the event that $R_{o d}^{j}$ can propagate the vulnerabilities is $\prod_{e \in R_{o d}^{j}} p_{e}$. So the probability that at least one edge in $R_{o d}^{j}$ cannot propagate is $\left(1-\prod_{e \in R_{o d}^{j}} p_{e}\right)$. Define the events $W_{o d}^{1}, W_{o d}^{2}, \cdots, W_{o d}^{M_{o d}}$ by

$$
W_{o d}^{j}=\left\{\text { at least one edge in } R_{o d}^{j} \text { cannot propagate }\right\}
$$

Therefore, the probability of $W_{o d}^{j}$ is

$$
P\left\{W_{o d}^{j}\right\}=1-\prod_{e \in R_{o d}^{j}}\left(1-p_{e}\right)
$$

Since $d$ will not be attacked by $o$ if and only if all of the events $W_{o d}^{j}$ occur, we have

$$
\begin{aligned}
1-p_{o d} & =P\left\{W_{o d}^{1} W_{o d}^{2} \cdots W_{o d}^{M_{o d}}\right\} \\
& =P\left\{W_{o d}^{1}\right\} P\left\{W_{o d}^{2} \mid W_{o d}^{1}\right\} \cdots P\left\{W_{o d}^{M_{o d}} \mid W_{o d}^{1} \cdots W_{o d}^{M_{o d}-1}\right\} \\
& \geq \prod_{j=1}^{M_{o d}} P\left\{W_{o d}^{j}\right\}
\end{aligned}
$$


Equivalently,

$$
p_{o d} \leq 1-\prod_{j=1}^{M_{o d}} P\left\{W_{o d}^{j}\right\}
$$

The last inequality above indicates the upper bound of $p_{o d}$. Replace $P\left\{W_{o d}^{j}\right\}$ according to Equation 3.16, we have

$$
p_{o d} \leq 1-\prod_{j=1}^{M_{o d}}\left(1-\prod_{e \in R_{o d}^{j}} p_{e}\right)
$$

Considering the lower bound and the upper bound, we have the following bounds for the function of propagated vulnerability:

$$
\prod_{i=1}^{D_{o d}}\left[1-\prod_{e \in C_{o d}^{i}}\left(1-p_{e}\right)\right] \leq p_{o d} \leq 1-\prod_{j=1}^{M_{o d}}\left(1-\prod_{e \in R_{o d}^{j}} p_{e}\right)
$$

Figure 3.5 shows the value changes between $p_{\text {od }}$ and the upper bound of $p_{\text {od }}$ when $\alpha=0.5$ and $p_{e}$ ranging from 0 to 0.2 . From the figure we can see that the upper bound is quite tight when $p_{e} \in[0,0.2]$, close to the actual value of $p_{\text {od }}$, which is close to the real-world scenario that $p_{e}$ cannot be too large $\left(p_{e}=0.2\right.$ is already overly vulnerable in reality). Effectively, we will be able to leverage this upper bound to estimate the worst case of propagated vulnerability.

By listing Algorithm 1, we have implemented the function to calculate the bounds of propagated vulnerabilities. It takes the topology of the ODG as the input. Then it uses standard algorithms to find $R_{o d}$ and $C_{o d}$. After that, we consider the vulnerabilities of all edges of all paths in $R_{o d}$ and $C_{o d}$ for the bounds Algorithm 1 outputs. The output value is the lower and upper bounds of the joint vulnerability of a path. 


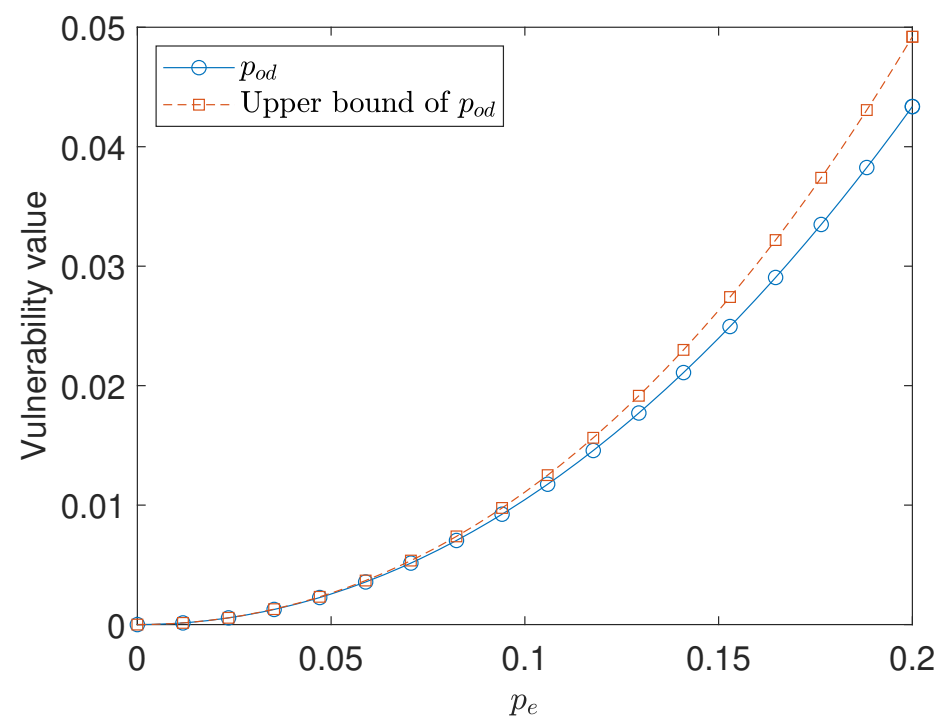

Figure 3.5: Comparison between the actual value of $p_{o} d$ and its upper bound. $\alpha=0.5$ and $p_{e} \in[0,0.2]$

We choose the upper bound during the calculation to estimate the path vulnerability aggressively. And then we finally get the propagated vulnerability between two objects.

\subsubsection{The Impact of Offloading on Object Vulnerability}

Now that the vulnerability of each object is available, it becomes easier to answer which objects should be kept locally and which can be offloaded. Simply keeping an object with highest vulnerability on the local device does not necessarily make it safer. Staying locally for an object reduces its cloud-originated vulnerability because no eyes would watch it directly from the cloud. But it does not reduce the propagated vulnerability. Meanwhile, separating objects working closely together may cause significant communication cost.

We choose to keep objects generating the greatest impact on other nodes. Those originators are kept on mobile devices rather than in the cloud. To calculate the 


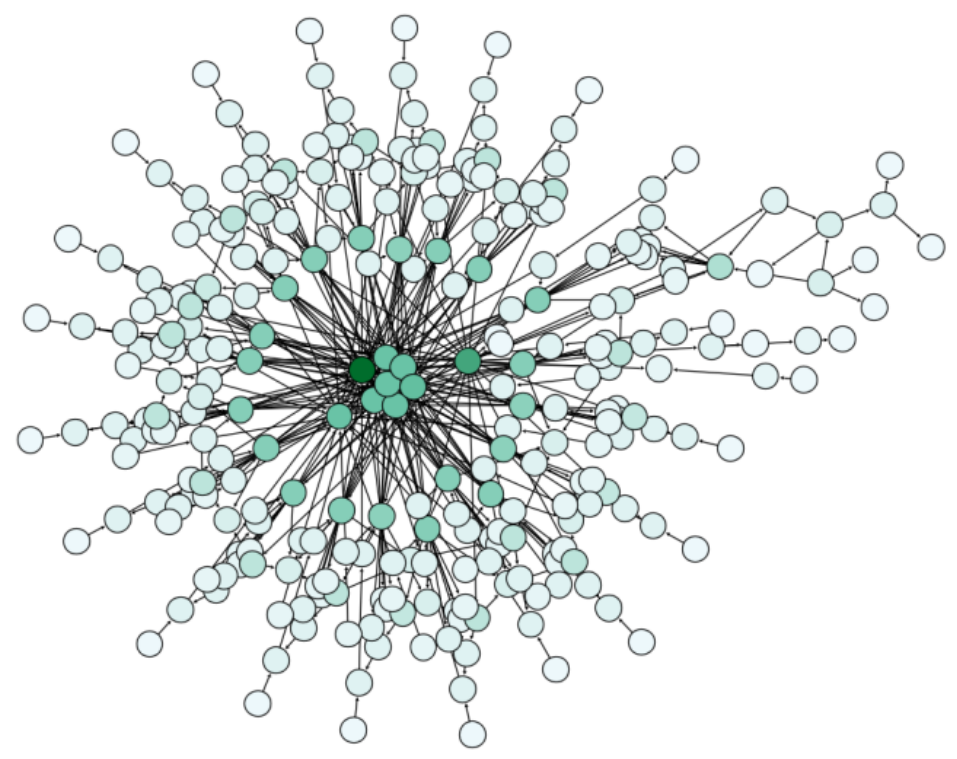

Figure 3.6: ODG of TrendCraw after the first piece of news has been retrieved

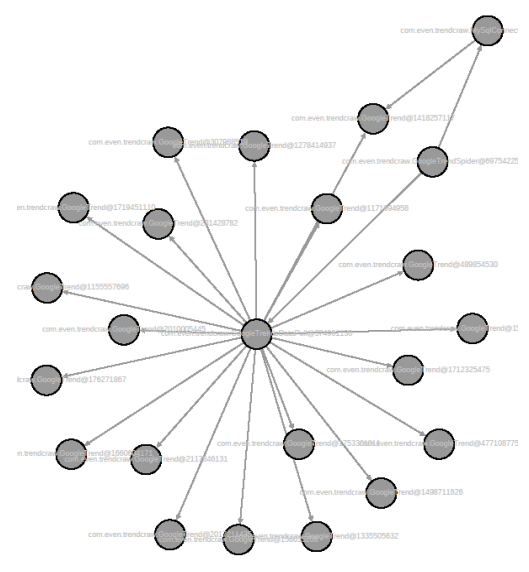

Figure 3.7: ODG of TrendCraw fetching with only original objects 
impact of each object $o$, we first calculate the difference between the vulnerability of each object with and without $o$ in the ODG, which is called the absolute impact. If the propagated vulnerability of the object $d$ is $\mu_{d}^{o}$ with $o$ and $\mu_{d}^{\bar{o}}$ without $o$, the absolute impact from $o$ to $d$, denoted by $\delta_{d}^{o}$, is then

$$
\delta_{d}^{o}=\mu_{d}^{o}-\mu_{d}^{\bar{o}}
$$

Then we can calculate the ratio $o$ accounts for the vulnerability of $d$ because the difference is solely caused by the removal of $o$. We name the ratio the relative impact

from $o$ to $d$, denoted by $\gamma_{d}^{o}$, where $\gamma_{d}^{o}=\frac{\delta_{o d}}{\pi_{d}}$. Finally, we average the relative impact of each node over all nodes and denote the result by $\Delta_{o}$, also known as the impact factor for the originator. Given that $|\mathbb{V}|$ is the total number of objects in the ODG, and $\Delta_{o}$ can be calculated by the equation below:

$$
\begin{aligned}
\Delta_{o} & =\frac{1}{|\mathbb{V}|} \sum_{d \in \mathbb{V}-o} \gamma_{d}^{o} \\
& =\frac{1}{|\mathbb{V}|} \sum_{d \in \mathbb{V}-o} \frac{\delta_{d}^{o}}{\pi_{o d}} \\
& =\frac{1}{|\mathbb{V}|} \sum_{d \in \mathbb{V}-o} \frac{\mu_{d}^{o}-\mu_{d}^{\bar{o}}}{\pi_{o d}} .
\end{aligned}
$$

The impact factor reflects what the vulnerability of a single object could bring to the ODG. Objects with larger impact factors should be kept running on the local mobile device so as to avoid its cloud-originated vulnerability.

\subsection{Evaluation and Numerical Results}

The ODG model has been evaluated with real apps to verify if vulnerable objects can be correctly identified. We try our best to keep our results general. In our current 


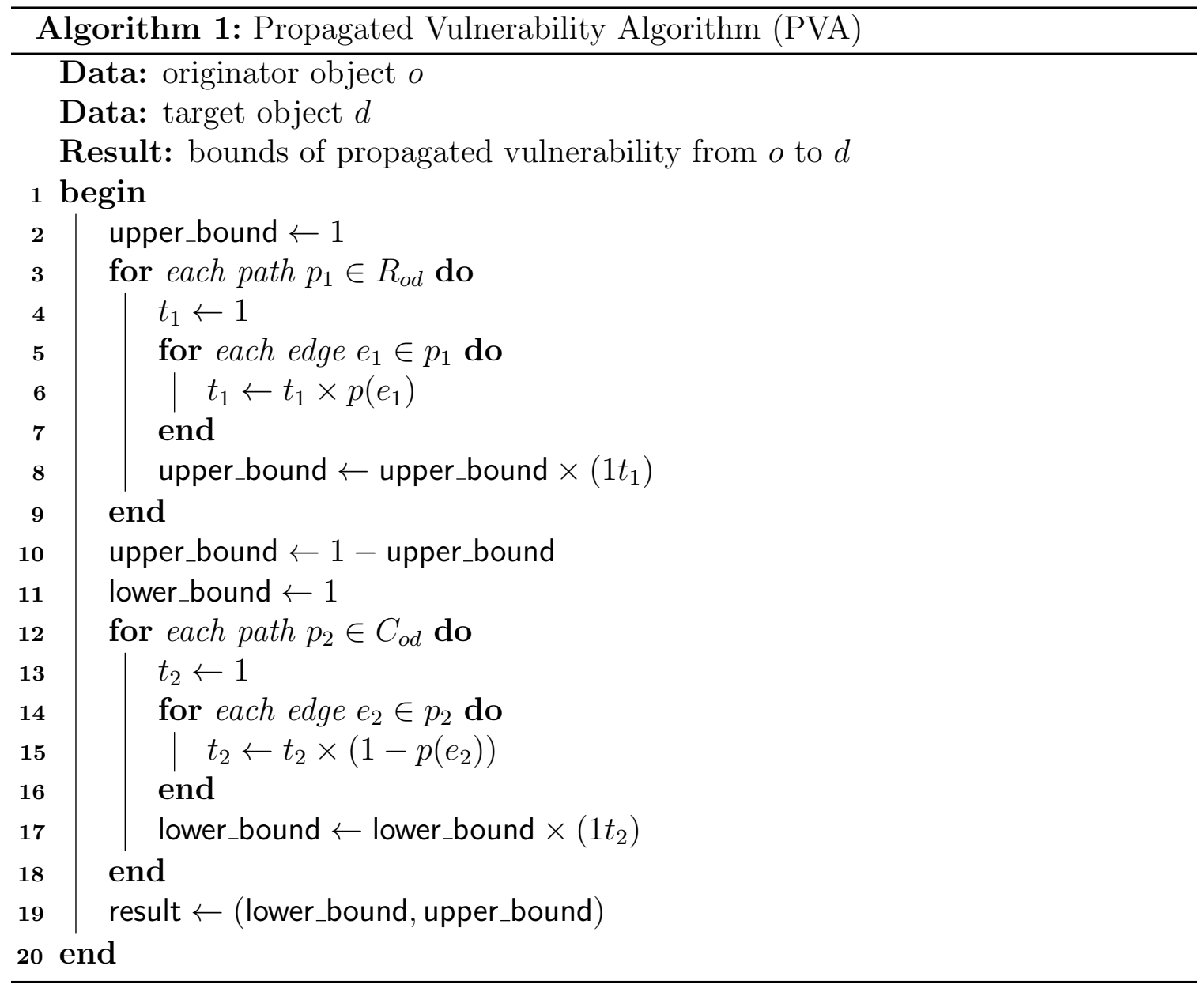

Table 3.1: Parameters of sample apps

\begin{tabular}{lllll}
\hline \multirow{2}{*}{$\begin{array}{c}\text { App } \\
\text { Name }\end{array}$} & \multicolumn{2}{c}{ From } & Original Packages & \multirow{2}{*}{ Imported } \\
\cline { 2 - 4 } & Packages & Classes & Activities & Packages \\
\hline TrendCraw & 2 & 9 & 3 & 183 \\
MyExpense & 3 & 24 & 11 & 194 \\
iMetro & 22 & 145 & 20 & 192 \\
\hline
\end{tabular}


experiments, we pick three open-source sample applications from different categories from Google Play store and third-party stores. They are listed as follows:

- TrendCraw fetches news feeds from the Internet periodically, and then displays the contents to users.

- MyExpense manages the daily expense of the user. It does not have Networkrelated actions.

- iMetro provides subway maps and station schedules of cities all over the world. It downloads subway information from the Internet according to users' selection.

Table 3.1 illustrates parameters of the sample apps. The packages written specifically for the apps (rather than imported) are called original packages, while those imported from other libraries are called imported packages. From the table, we notice the significant difference between the numbers of original packages and those of imported ones. We assume that impacts of imported packages are merged into cloud-originated vulnerabilities so that we can focus on a relatively smaller number of objects. Figure 3.6 and Figure 3.7 compare the differences of the two methods. This simplification is for illustration purpose only and it does not compromise the generality of our approach. We treat the vulnerability analysis of imported packages as separated tasks. After the vulnerabilities of all imported packages are known, we are able to calculate the vulnerability of the package that imports those packages. Moreover, we build a database storing the vulnerabilities of imported packages. The vulnerabilities of the widely-used packages only needs to be computed once. The later requests can directly use the existing results of vulnerabilities of the imported packages. 


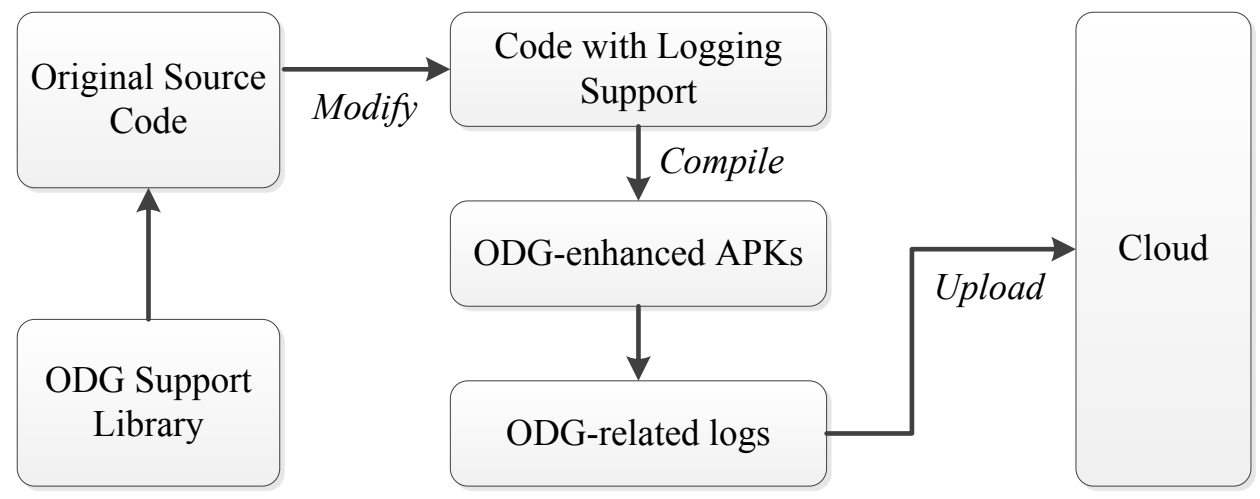

Figure 3.8: ODG generation process on existing open-source Android apps

\subsubsection{Standard Process of Experiments}

In our experiments, we import both the source code of the apps and our library supporting the ODG model. Minor changes are made to the original source code to enable logging the object calls. Then we recompile all apps to generate the ODGenhanced Android installation package files in format of .APK, and copy them to smartphones. Our library enables the app to log every object and the call relationships it creates dynamically at runtime, and to save the file in its external storage card. The flow chart of the standard process is shown in Figure 3.8 .

There are two conditions to make the experiments practical. One is to keep the generality of the object call relationship logging method. The other is to ensure the acceptable complexity of the offloading decision algorithm. Our solution satisfies the conditions as below:

To keep the generality of our process so that fewer changes are required to apply the ODG model to a new app, we introduce the aspect-oriented programming (AOP) paradigm 102 to enable logging right before the occurrence of ODG-related events, such as method calls and object creations. The AOP intercepts the built-in lifecycles of the app rather than its specific working flow. Therefore it is easy to implement automated analysis for apps with the ODG model. 


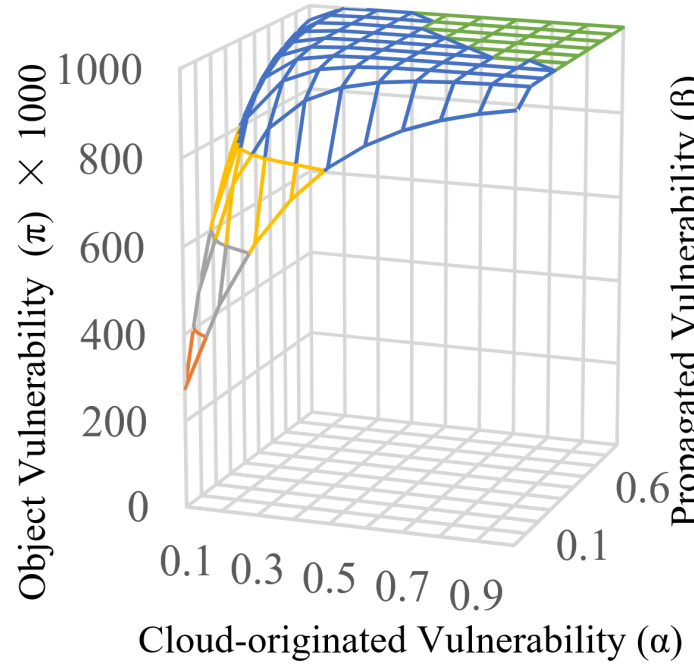

(a) RenderProgram

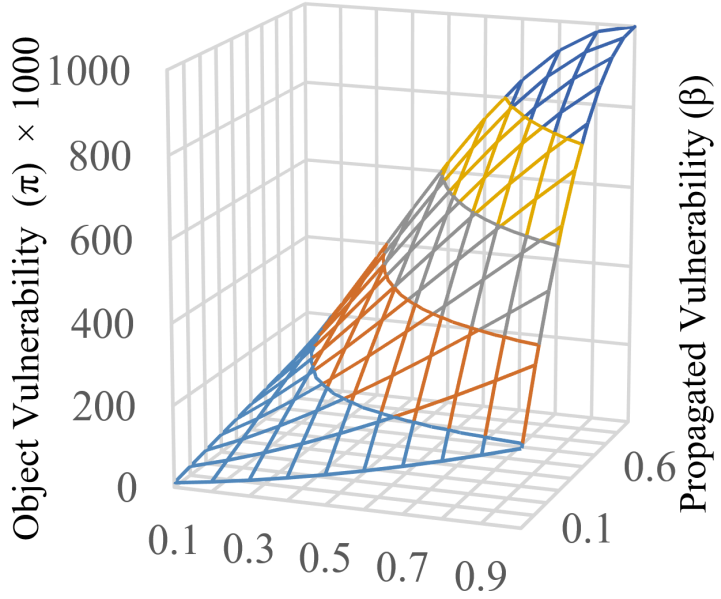

Cloud-originated Vulnerability $(\alpha)$

(b) RenderStation

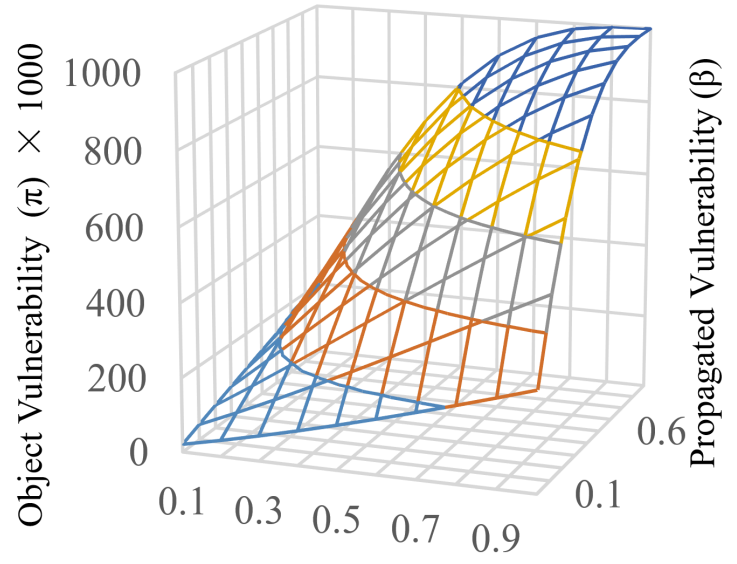

Cloud-originated Vulnerability $(\alpha)$

(c) RenderStationName

Figure 3.9: Vulnerabilities of three objects under different cloud-originated vulnerabilities $(\alpha)$ and propagated vulnerabilities $(\beta)$

\subsubsection{Experimental Results}

In order to evaluate the offloading results of our ODG model, we implement two other offloading analytical models: CloneCloud (CCD) [3] and ThinkAir (TAR) [4]. The execution time, energy cost and security levels of the three models are compared. 


\section{Offloading Algorithm Execution Time}

In our analysis on the sample apps, it takes more time than the CCD and TAR mechanisms to perform one round of offloading decisions with the strategies above applied, which is shown in Table 3.2 .

Table 3.2: Time taken for analyzing apps with the three offloading mechanisms

\begin{tabular}{llll}
\hline \multirow{2}{*}{ App } & \multicolumn{3}{c}{ Offloading Analysis Time (ms) } \\
\cline { 2 - 4 } Name & CloneCloud & ThinkAir & ODG \\
\hline TrendCraw & 20937 & 18561 & 22428 \\
MyExpense & 37115 & 33753 & 40756 \\
iMetro & 61512 & 54917 & 65970 \\
\hline
\end{tabular}

\section{Impact of System-wide Vulnerability Change}

When all objects are running in the cloud, we wonder which objects are more sensitive to system-wide vulnerability hike, because cloud-originated vulnerabilities change jointly when they run in different clouds. Propagated vulnerabilities also change together depending on the strength of attacks. As a panorama of the response to system-wide vulnerability change, Figure 3.9 shows the vulnerabilities of objects in the sample app iMetro with different $\alpha$ and $\beta$. The vulnerabilities are categorized by the types of objects, i.e., the classes of objects.

From the figure we can conclude that the first object org.ametro.render.RenderProgram has its vulnerability increased much faster than the other two objects. That indicates its higher sensitivity to the system-wide vulnerability change. Checking the causes of the fact, we have noticed different numbers of neighbors for the listed objects. The objects with larger number of neighbors, including one- and two-hop neighbors, tend to have higher vulnerabilities because they accumulate more propagated vulnerability. Combine Figure 3.9 with Figure 3.10, we can infer the positive correlation between the sum of one-/two-hop 
neighbors of one object and its propagated vulnerability.

To demonstrate the impact of object vulnerabilities to offloading decisions in the ODG model, the same numbers of objects are offloaded in each of the three models for comparison. We show results for the energy cost on smartphones and the number of sensitive APIs called in the cloud below.

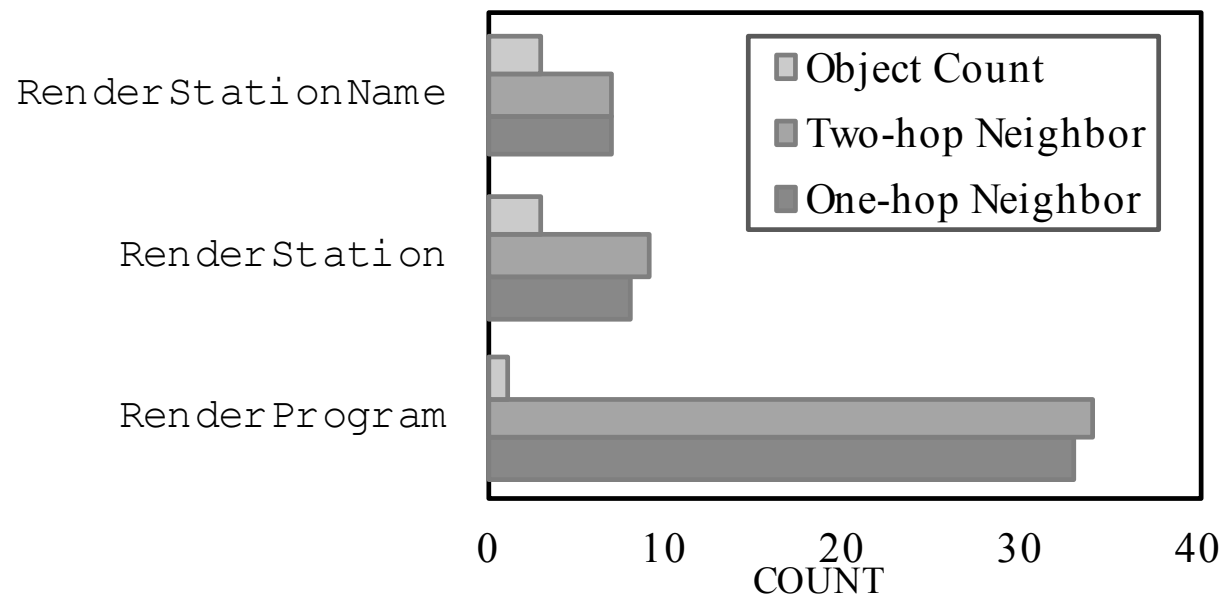

Figure 3.10: Number of one- and two-hop neighbors may impact the sensitivity of objects to system-wide vulnerability change

\section{Phone-side Energy Cost}

As one of the primary goals of offloading, the energy costs of the three apps are measured. Since the goal of energy saving and secure offloading are conflicting, we choose the security factor as priority in our ODG model: the object with the least security impact shall be offloaded first. When the security impact of the objects is the same, we choose the one that can save most energy for offloading. Meanwhile, the energy factor is taken as the ending condition in our experiments: for all offloading mechanisms, at most $14 \%$ of the total objects are offloaded. We choose the model PowerTutor [102] as the measurement tool of energy cost at the smartphone side. After normalizing the energy cost data, the changes of costs are illustrated in Figure 


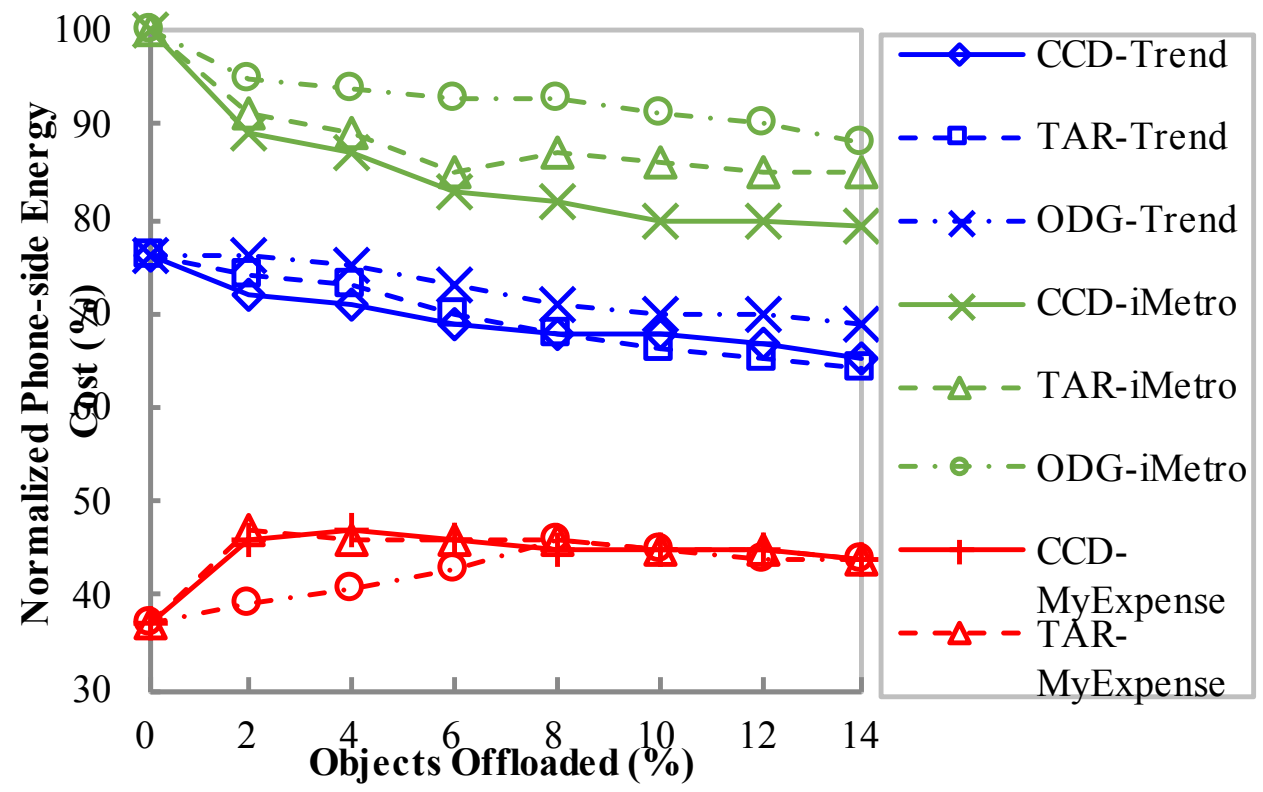

Figure 3.11: Normalized phone-side energy cost with the three offloading schemes under different percentages of objects offloaded

3.11. We notice that the ODG model consumes more energy at the smartphone side comparing with the other two mechanisms. But it can still save around $10 \%$ of the energy with $14 \%$ of the objects offloaded.

\section{Sensitive APIs Accessible by the Cloud}

We choose to trace the running locations of sensitive APIs related to retrieving users personal information, phone identities and geographical data, because they are related to security issues of mobile apps. We take the approach from [103] to locate the sensitive APIs and analyze the offloading results from the same experiment groups conducted in last section. In our case, an API will be marked as vulnerable either when they run in the cloud or when they can be accessed from the cloud due to object dependencies. From Figure 3.12 the ODG model exposes significantly less sensitive APIs to the cloud for all three sample apps. 


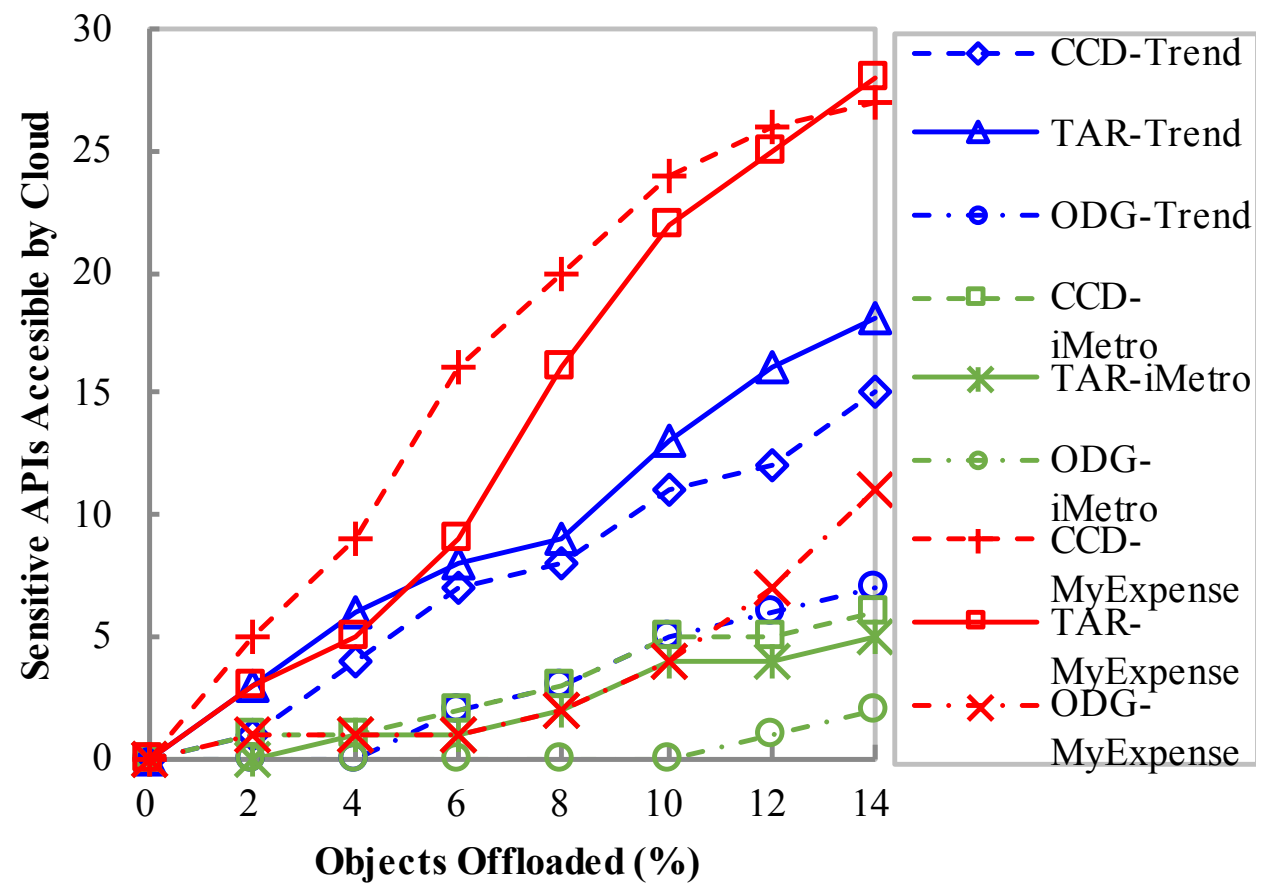

Figure 3.12: Numbers of sensitive APIs accessible by the cloud

\subsection{Chapter Summary}

In this chapter, we focus on offloading apps securely without putting vulnerable parts in the cloud. A model named the object dependency graph (ODG) is proposed to analyze the security of mobile apps according to objects they have created at runtime. With the knowledge of cloud-originated and propagated vulnerabilities of runtime objects, the objects vulnerabilities are calculated taking into account the multipath vulnerability propagation. We have applied our model to real Android apps and have shown that compared to other two existing offloading mechanisms, our offloading approach yields more secure results. 


\section{Chapter 4}

\section{Availability-aware Application Placement}

\subsection{Introduction}

Mobile edge computing (MEC) is taking Network Function Virtualization (NFV) to the end users closer than ever [5 7]. Instances of edge computing, including regional datacenters [8], cloudlets [1], and fog nodes [9], deliver highly-responsive cloud services at the network edge. As a key technology towards 5G, MEC architecture proposed by ETSI [7] leverages existing NFV frameworks widely adopted by carriers and vendors [10,11]. Elastic mobile edge applications, including network services, are deployed close to the user equipment (UE) with low latency. UE is any device used directly by an end-user to communicate. Both UE application providers and telecommunication service providers (TSPs) can take advantage of MEC to reduce cost and to adjust services with agility based on fast-changing user demands.

A mobile edge application consists of one or more collaborating virtual machines (VMs). It is of paramount importance to maintain the high availability of mobile edge applications. Compared to centralized datacenters used by public cloud, MEC hosts are heterogeneous with varying computing, storage and networking capabilities [18. Smaller scale private cloud servers can be deployed near their designated groups of 
users as MEC hosts, the characteristics of which lead to the following indications:

(i) A single MEC server deployment is less powerful compared to the highlyavailable, centralized cloud as it serves a smaller group of users within the base station's coverage. It can be a micro datacenter which is unlikely to merit its own security guard or have the same level of redundancy as a larger facility [104.

(ii) The offloading nature of MEC brings higher system complexity that can jeopardize the availability 19$]$.

(iii) Service function chaining (SFC) is possible on MEC servers, as videos, augmented reality data, location-based services, and other computational-intensive tasks can take a chain of services to process.

These facts conclude that the hosts used in MEC are less reliable with lower availability. When mobile edge applications run in MEC servers, they must be protected from service outage due to host failure.

To maximize the availability while maintaining costs and latencies at acceptable levels, placement rules often come into play to tune the performance and security of a mobile edge application 21]. In practice, placement rules mainly refer to the affinity and anti-affinity rule [22]. A group of VMs with the affinity rule applied must be deployed on the same host. On the contrary, the anti-affinity rule to a group of VMs ensures that each VM in the group is deployed on a different host.

The affinity rule helps reduce communication costs between VMs serving the same mobile edge application: VMs on the same host connect to each other using virtual networks private to the host and require no physical networking infrastructure. Samehost network traffic essentially takes up computational resources of the host and has better performance than physical networks. This becomes handy especially when frequent inter-VM communications are needed. An obvious down side of the affinity 


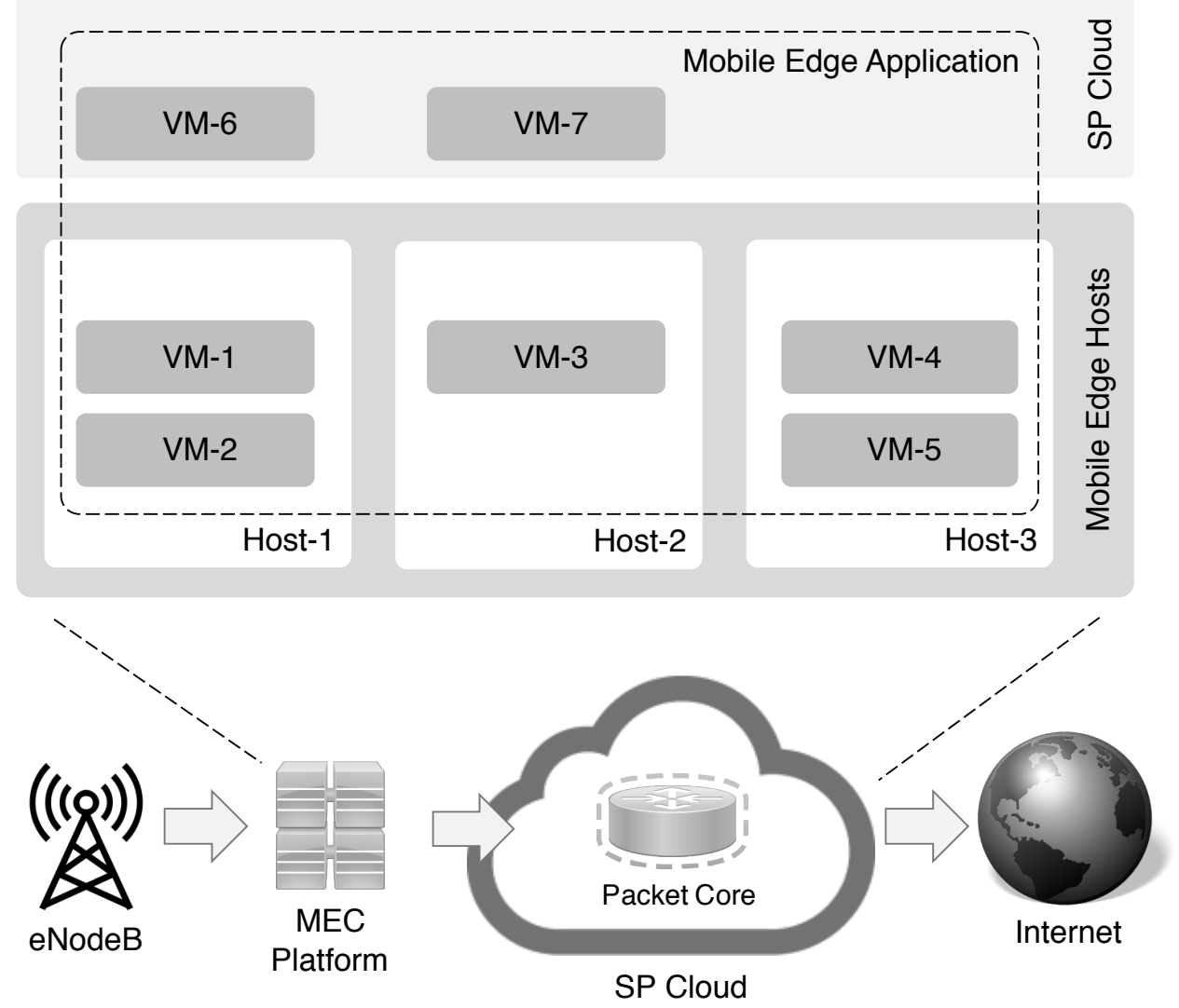

Figure 4.1: A mobile edge application deployment with host placement rules.

rule is putting all eggs in one basket. If the host is down, the entire mobile edge application would be out of service. Resource contention is another drawback due to oversubscription, which is typically configured for maximizing host resource utilization [105]. Too many resource-thirsty VMs packed together would overload their host.

In comparison, anti-affinity rules are ideal for High Availability (HA). If multiple VMs of the same type are deployed on different hosts, having one host down would not take all instances out of service. Therefore, the mobile edge application can still be functional. Fig. 4.1 demonstrates an example of a mobile edge application deployment across multiple hosts to increase availability. There are five VMs deployed in three groups with each group placed on a separate host. A minimum of three 
VMs are required for the application. The placement will ensure the application is in service if one host is down. On the other hand, enforcing the anti-affinity rule for resource-intensive VMs can reduce chances of the hosts being overloaded due to oversubscription. As a trade-off, the mobile edge application using anti-affinity rule would lose the benefits of low communication costs and higher confidentiality brought by co-location.

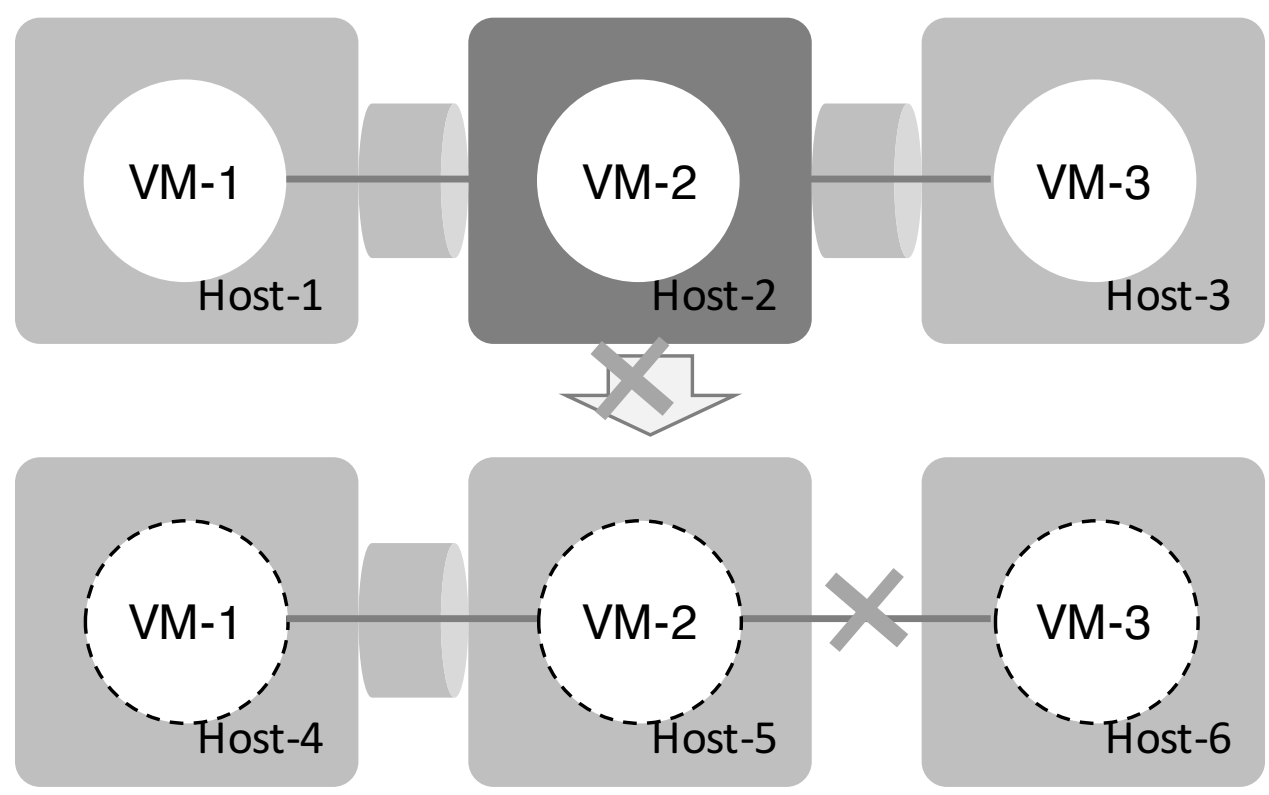

Figure 4.2: An attempt to migrate a service chain without enough resource.

Besides hosts availability, Service Function Chaining (SFC) [20] is commonly adopted to formulate a network function with complete features to provide end-toend service. Similarly, SFC can be required for a mobile edge service provisioned by multiple chained functions. Different functions, i.e., VMs deployed on hosts, must be chained together to process a stream of requests. Therefore, network topology and its availability can become the primary bottleneck of the mobile edge service. When a link is down, functions connected by that link will need to migrate to recover from the link outage. For instance, when the SFC is formulated by L2 switching, the open vSwitch (OVS) will be responsible for switching traffic among the functions, which 
means all applications can only be deployed on the same host. The outage of the virtual switch would lead to the migration of all functions to a different host. Therefore, it is more difficult to migrate a mobile edge application considering SFC. Fig. 4.2 shows an example of attempt to migrate a service chain due to Host 2's outage. The service chain consists of 3 VMs and two links using L2 switchingWhen Host-2 is down, the service chain has to be migrated to three hosts with the same topology. Host-4, Host-5, and Host-6 have enough resources to have the service chain deployed. However, the topology is not identical. Therefore, the attempt to migrate the service chain will not succeed.

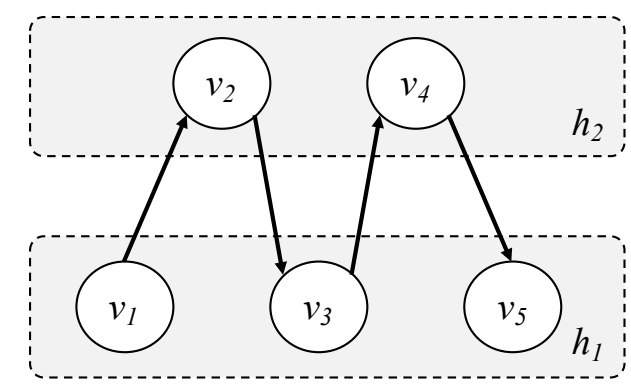

(a)

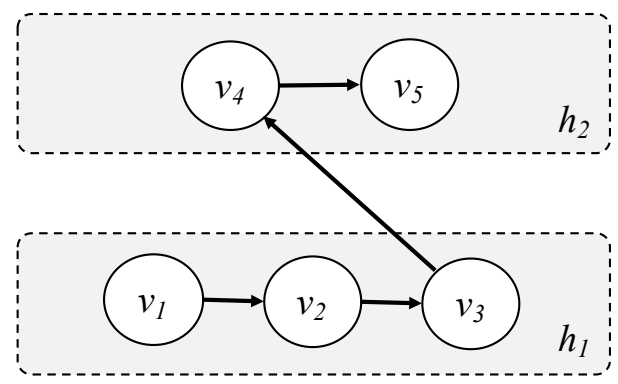

(b)

Figure 4.3: Inefficient partitioning of VMs for a mobile edge application.

Enforcing SFC can also cause massive increase of latency if the placement strategy is not aware of the chaining policy. As demonstrated in Fig. 4.3(a), inefficient partitions of VMs with SFC applied can cause traffic going back and forth among hosts, resulting in excessive latency in comparison with Fig. 4.3(b).

Considering placement constraints of host availability, SFC, and the limited resources at the mobile edge, it can be foreseen that when mobile edge hosts are unavailable, mobile edge application VMs might not be able to continue service if they are kept at the edge. To maintain the desired availability, the more reliable, centralized cloud can come to the picture to coordinate with the edge. 
With the host placement rules and the link availability requirements, in this chapter, our interest is in finding adaptive placement strategies for different types of mobile edge applications to achieve lower costs, while still satisfying the availability and confidentiality requirements. Compared to existing work, our contributions include the following:

(i) We address the application availability concerns even when some mobile edge hosts are down. We believe at the network edge, hosts are less reliable and availability issues of MEC applications need more attention.

(ii) We consider the link health between hosts to support SFC. Individual VMs will be migrated when one or more links are down to maintain the service of the SFC-enabled mobile edge application.

(iii) A cost model is built considering the factors of inter-host traffic and resource overcommitting, to balance the load without causing explosive traffic between hosts.

(iv) We formulate a stochastic programming problem to minimize the cost based on our cost model, while maintaining the availability requirements.

(v) A heuristic algorithm, namely EdgePlace, is developed to return suboptimal results as the problem scales. Numerical results show the effectiveness of EdgePlace.

We divide the contents into the three following sections. Section 4.2 formulates the problem. Then the experimental results are shown in Section 4.3. Section 4.4 provides a summary of this chapter. 


\subsection{Problem Formulation}

We formulate the problem in this section and the definitions of symbols can be found in List of Symbols. Suppose a mobile edge application has a set of elastic group of VMs, denoted by $\mathbb{V}$, to be deployed on a MEC virtualization infrastructure (MECVI) with a set of hosts $\mathbb{H}$. VMs can be deployed on any of the hosts available from the MECVI, or on the remote cloud, denoted by $c$. Assume that there be $N_{V}$ VMs used by the mobile edge application, with each VM denoted by $v$, and $N_{H}$ hosts in the MECVI, with each host denoted by $h$. Different hosts are connected to each other by network links. We denote the network link between Hosts $h_{i}$ and $h_{j}$ as $e_{i j}$.

Define an assigning function $x_{v h}$, whose value is 1 if $\mathrm{VM} v$ is assigned to Host $h$, 0 otherwise. For the cloud, a similar assigning function $x_{v c}$ is defined to be 1 if $v$ is deployed on $c$ and 0 if not.

$$
\begin{aligned}
& x_{v h}= \begin{cases}1, & v \text { is deployed on } h ; \\
0, & \text { otherwise. }\end{cases} \\
& x_{v c}= \begin{cases}1, & v \text { is deployed on the cloud; } \\
0, & \text { otherwise. }\end{cases}
\end{aligned}
$$

\subsubsection{Availability of Elastic Mobile Edge Applications}

Let the minimum number of VMs required by the mobile edge application be denoted by $N_{m}$. Similar to virtual network function $(\mathrm{VNF})$ resource management in service chaining [106], if the number of available VMs is at least $N_{m}$ and they are connected according to the designed topology, the mobile edge application is then considered in service. Otherwise, it is deemed down as it would not satisfy SLA requirements for the volume of requests. 
An intuitive way to increase the availability of the mobile edge application is VM redundancy. In production environments, for example, it is quite common to keep a certain number of VMs of the same type running with the configuration of Keepalived [107], to maintain the service availability or to balance the load. Thanks to the elasticity of the mobile edge application, auto-scaling is enabled in form of deploying extra VMs, so that even if some VMs are down, there are still more than $N_{m}$ VMs in service. There must be $N_{V} \geq N_{m} \geq 0$. On the other hand, it is not as easy to increase link redundancy: substrate network links between hosts are pre-created and there isn't always a match for a whole application with SFC.

To keep a mobile edge application up, the availability of both the VMs and the links is required. Consider the failure points of these two factors, there are three situations in our discussion that can lead to VM service outage:

* Application internal failure. If the software installed crashes or hangs, the service provided by the VM would be unavailable. Internal failure on one VM is assumed to be independent from those on other VMs. Denote the probability that a VM is working without internal failure as $P_{V}$.

* Host failure. If one host is down, all VMs deployed on it would be out of service. Denote the probability that a host will not fail as $P_{H}$.

* Link failure. A link may experience technical issues, either due to a software bug, or substrate network outage. If a link used by a VNF is down, it may affect the availability of the VNF.

Define $\hat{v}_{h}$ as the total number of VMs assigned to host $h$, and $\hat{v}_{c}$ as the number of VMs assigned to the cloud. Then we have

$$
\hat{v}_{h}=\sum_{v} x_{v h}, \quad \hat{v}_{c}=\sum_{v} x_{v c}
$$


Let $A_{h}$ be the random variable denoting the number of VMs to be available on a host $h$. Because different VMs on the same host fail independently due to internal failure, by binomial distribution, we have

$$
p_{a_{h}} \triangleq \operatorname{Pr}\left\{A_{h}=a_{h}\right\}=\left(\begin{array}{c}
\hat{v_{h}} \\
a_{h}
\end{array}\right) P_{V}^{a_{h}}\left(1-P_{V}\right)^{\hat{v}_{h}-a_{h}} P_{H}
$$

By assumption, different hosts also fail independently. We have

$$
p_{\bar{a}} \triangleq \operatorname{Pr}\left\{A_{1}=a_{1}, A_{2}=a_{2}, \ldots, A_{H}=a_{H}\right\}=\prod_{h} p_{a_{h}}
$$

The additional condition for all the VMs to be available to the mobile edge application is to ensure that the links among these VMs are all available, too. Define $p_{E}(\bar{a})$ as the probability that all hosts with one or more VMs deployed are connected to each other. Also, define $P_{E}\left(e_{i j}\right)$ as the probability that $e_{i j}$ is up and available. We have

$$
p_{E}(\bar{a}) \triangleq \prod_{a_{h_{i}}, a_{h_{j}}>0, a_{h_{i}} \neq a_{h_{j}}} P_{E}\left(e_{i j}\right) .
$$

Define $p_{y}$ as the probability that there are at least $y$ VMs available and $\hat{a}=\sum_{h} a_{h}$. Meanwhile, all hosts with VMs deployed must be able to communicate to each other. We have

$$
\begin{aligned}
p_{y} & \triangleq \operatorname{Pr}\left\{\sum_{h} A_{h} \geq y-\hat{v}_{c}\right\} p_{E}(\bar{a}) \\
& =\sum_{\bar{a}, \hat{a}=y-\hat{v}_{c}}^{N_{V}} p_{\bar{a}} \prod_{a_{h_{i}}, a_{h_{j}}>0, a_{h_{i}} \neq a_{h_{j}}} P_{E}\left(e_{i j}\right) \\
& \geq 1-\eta,
\end{aligned}
$$

where $\bar{a}=\left(a_{1}, a_{2}, \ldots, a_{H}\right)$ and $\eta$ is a small positive number denoting the maximum 
failure probability allowed.

\subsubsection{Inter-host Link Availability and Importance Factor}

Inter-host link availability is a fundamental part to ensure the availability of the mobile edge application. An event of a key inter-host link outage is catastrophic: even if all individual VMs are running, the traffic would not be able to flow through between one or more pairs of VMs and the SFC would not be functional. For each inter-host link $e_{i j}$, there can be one or more inter-VM links sharing its bandwidth. Link outages require migrating the mobile edge application VMs if the network links cannot be fixed in time. Therefore, link availability has significant influence on possible VM migrations and costs incurred.

The link importance factor of an inter-host link $e_{i j}$, denoted by $I_{L}\left(e_{i j}\right)$, describes how important an inter-host link $e_{i j}$ is for the application availability. We determine the importance of each host link by the two parameters below.

The first parameter is the indicator of a link between two individual VMs, denoted by $L\left(v_{h_{i}}, v_{h_{j}}\right)$, such that

$$
L\left(v_{h_{i}}, v_{h_{j}}\right)= \begin{cases}1, & \text { there is traffic between } v_{h_{i}} \text { and } v_{h_{j}} \\ 0, & \text { otherwise }\end{cases}
$$

The more inter-VM links an inter-host link carries, the more vital it becomes. The reason behind this ranking parameter is the potential consequence of migration: failure of an inter-host link used by many VMs would lead to massive migration of all VMs connected by that inter-host link, which would be more disruptive to the service. When placing VMs, more reliable host links should be picked if it will be intensively shared. 
The other parameter is $B_{V}\left(e_{i j}\right)$, which is the total bandwidth consumed by traffic between VMs on the two hosts. It is selected because larger bandwidth usages would cause challenges at the time of migration: it can be hard to find another link with enough capacity.

$$
B_{V}\left(e_{i j}\right)=\left[\sum_{v_{h_{i}}, v_{h_{j}}, h_{i} \neq h_{j}} B\left(v_{h_{i}}, v_{h_{j}}\right)\right]
$$

Combining the two parameters, we define the link importance factor of a host link $e_{i j}$, denoted by $I_{L}\left(e_{i j}\right)$, as the number of virtual links between two hosts times the traffic flowing through the link:

$$
I_{L}\left(e_{i j}\right)=\frac{\left[\sum_{v_{h_{i}}, v_{h_{j}}, h_{i} \neq h_{j}} L\left(v_{h_{i}}, v_{h_{j}}\right)\right]}{a_{i j}} \frac{B_{V}\left(e_{i j}\right)}{B\left(e_{i j}\right)},
$$

where $a_{i j}$ is the maximum number of virtual links possible on $e_{i j}$. Therefore, $I_{L}\left(e_{i j}\right) \in$ $[0,1]$. The value of $I_{L}\left(e_{i j}\right)$ will rise to mark up a link's importance given it is either occupied by more pairs of VMs, or there is more traffic assigned to $e_{i j}$, or both.

\subsubsection{Inter-host Network Bandwidth Costs}

High availability comes at a cost: extra resources are used for hosting VMs, and extra traffic occurs between VMs. The traffic between VMs on the same host will be processed by the CPU of the host without going through the actual physical network. In this section, we temporarily ignore the cost of intra-host traffic. It will be discussed in the next section as part of the CPU cost.

Let $v_{h}$ represent a VM deployed on Host $h$, i.e., $x_{v_{h} h}=1$. Consider a VM $v_{h_{i}}$ on Host $h_{i}$ sends traffic to another $\mathrm{VM} v_{h_{j}}$ on Host $h_{j}$. We model the bandwidth 
demand for every two VMs in the mobile edge application, rather than simply viewing VMs on the same host as a cluster. This is due to SFC: even if two VMs are on the same host, there can be no traffic between them as they may not be next to each other in the chain. Let $B\left(v_{h_{i}}, v_{h_{j}}\right)$ be the total bandwidth demand from $v_{h_{i}}$ to $v_{h_{j}}$. If there is too much inter-host traffic, the networks would become congested and fail the mobile edge application. The required traffic throughput between two hosts must not exceed the designed bandwidth for the inter-host network. We define the total bandwidth of $e_{i j}$ by $B\left(e_{i j}\right)$ and its residue bandwidth by $R_{B}\left(e_{i j}\right)$. We calculate the residue bandwidth as following:

$$
R_{B}\left(e_{i j}\right)=B\left(e_{i j}\right)-B_{V}\left(h_{i}, h_{j}\right)
$$

Let $w_{B}\left(e_{i j}\right)$ stand for the unit cost of consuming the bandwidth of $e_{i j}$. We model $w_{B}\left(e_{i j}\right)$ to be inversely proportional to $R_{B}\left(e_{i j}\right)$ with the constant of proportionality $W_{B}$. Such model will favor choosing those links among hosts with more residual bandwidths and therefore will achieve balancing bandwidth consumption across the links between hosts. This reduces the risk of increasing delay due to link congestion.

Define the bandwidth cost of $e_{i j}$ as $S_{B}\left(e_{i j}\right)$. Additionally, we model $S_{B}\left(e_{i j}\right)$ to be inversely proportional to the availability of $e_{i j}$, which factors in the potential cost to migrate the SFC in event of a link failure: the more likely the link is failing, the higher price it would cost to use that link. Then we have

$$
\begin{aligned}
S_{B}\left(e_{i j}\right) & =\frac{B_{V}\left(e_{i j}\right) w_{B}\left(e_{i j}\right) I_{L}\left(e_{i j}\right)}{P_{E}\left(e_{i j}\right)} \\
& =\frac{B_{V}\left(e_{i j}\right) W_{B}}{R_{B}\left(e_{i j}\right)} \frac{\left[\sum_{v_{h_{i}}, v_{h_{j}}, h_{i} \neq h_{j}} L\left(v_{h_{i}}, v_{h_{j}}\right)\right]}{a_{i j} P_{E}\left(e_{i j}\right)} \frac{B_{V}\left(e_{i j}\right)}{B\left(e_{i j}\right)} \\
& =\frac{B_{V}^{2}\left(e_{i j}\right) W_{B}\left[\sum_{v_{h_{i}}, v_{h_{j}}, h_{i} \neq h_{j}} L\left(v_{h_{i}}, v_{h_{j}}\right)\right]}{B\left(e_{i j}\right)\left[B\left(e_{i j}\right)-B_{V}\left(e_{i j}\right)\right] a_{i j} P_{E}\left(e_{i j}\right)+\delta}
\end{aligned}
$$


where $\delta$ is a small positive number to avoid dividing by zero.

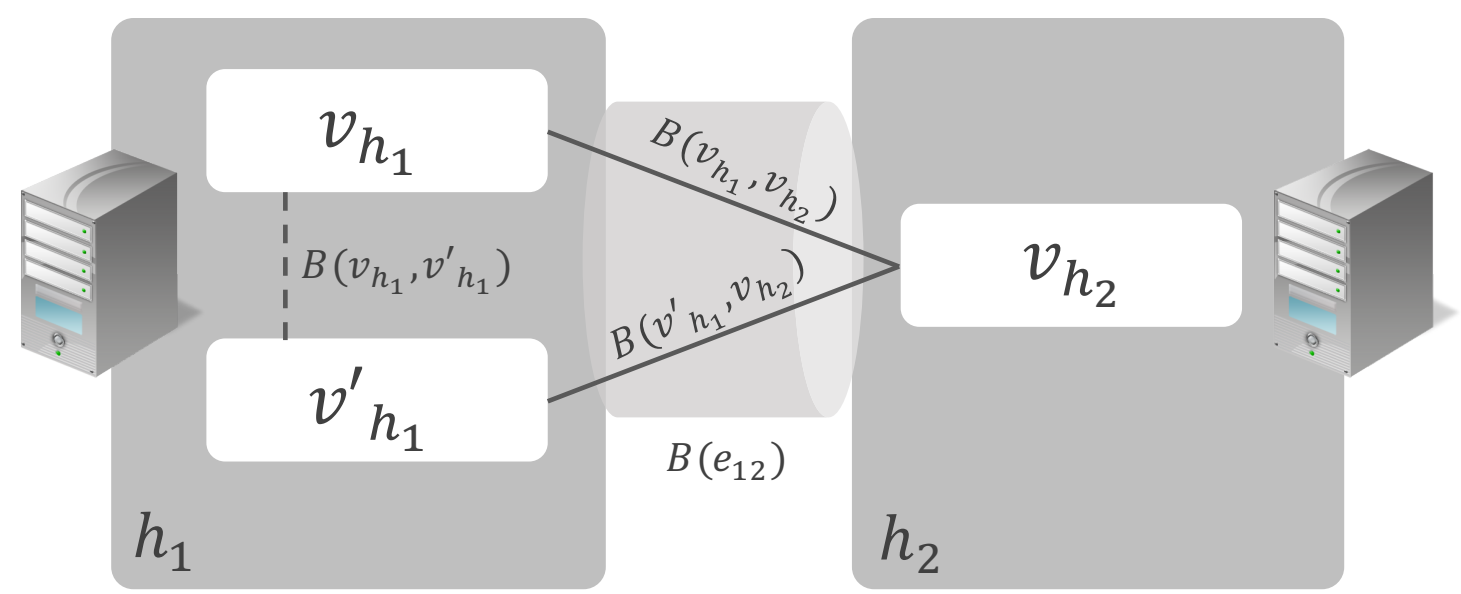

Figure 4.4: Inter-host and intra-host traffic between two VMs

\subsubsection{CPU and Memory Costs}

Consider any two VMs of the same mobile edge application. When they coordinate with each other, they will leave $\mathrm{CPU}$ and memory footprints on the host(s). If they are on two separate hosts $h_{i}$ and $h_{j}$, the networking costs are reflected by $S_{B}\left(e_{i j}\right)$ as shown in last section. If they are on the same host, no bandwidth cost will incur. However, splitting workloads across VMs on the same host also consumes resources. Instead of bandwidth, it costs extra host CPU and memory resources by running on virtual networks. Fig. 4.4 shows examples of costs from both inter- and intra-host traffic between two VMs. Inter-host network bandwidth consumptions $B\left(v_{h_{1}}, v_{h_{2}}\right)$ and $B\left(v_{h_{1}}^{\prime}, v_{h_{2}}\right)$ take up the bandwidth of $e_{12}$ and incur bandwidth costs. Intra-host network bandwidth consumption $B\left(v_{h_{1}}, v_{h_{1}}^{\prime}\right)$ consumes extra vCPU and memory of $h_{1}$ and incurs vCPU and memory costs.

Define the number of vCPUs required by $v$ as $C(v)$. It can be divided into two parts. One fixed part is to complete its own tasks, denoted by $C_{v}$. The other part is to coordinate with other VMs. Let the number of vCPUs required to coordinate with 
other VMs be proportional to the number of VMs to communicate with constant $\gamma_{C}$. We have $C(v)=C_{v}+\gamma_{C}\left(N_{V}-1\right)$.

Let the conversion ratio from the intra-host unit network bandwidth usage to the unit CPU usage denoted by $\alpha_{C}$. Then define $C_{h}$ as the total capacity of vCPUs for Host $h$. The remaining number of vCPUs, $R_{C}(h)$, can be calculated by

$$
R_{C}(h)=C_{h}-\sum_{v_{h}} C\left(v_{h}\right)-\alpha_{C}\left[\sum_{v_{h}, v_{h}^{\prime}, v_{h} \neq v_{h}^{\prime}} B\left(v_{h}, v_{h}^{\prime}\right)\right] .
$$

Let $w_{C}(h)$ stand for the unit cost of consuming the CPU resource of $h$. We model $w_{C}(h)$ to be inversely proportional to the remaining vCPUs with constant of proportionality $W_{C}$. Define the CPU cost of $h$ as $S_{C}(h)$. We have

$$
S_{C}(h)=\sum_{v_{h}} C\left(v_{h}\right) w_{C}(h)=\sum_{v_{h}} \frac{C\left(v_{h}\right) W_{C}}{R_{C}(h)+\delta},
$$

where $\delta$ is a small positive number to avoid dividing by zero.

Similar to the CPU cost, define the amount of memory needed by $v$ as $M(v)$. It can be divided into two parts. One fixed part is to complete its own tasks, denoted by $M_{v}$. The other part is to coordinate with other VMs. Let the amount of memory required to coordinate with other $\mathrm{VMs}$ be proportional to the number of $\mathrm{VMs}$ to communicate with constant $\gamma_{M}$. We have $M(v)=M_{v}+\gamma_{M}\left(N_{V}-1\right)$.

Let the conversion ratio from the intra-host unit network bandwidth usage to the unit memory usage denoted by $\alpha_{M}$. Then define $M_{h}$ as the total amount of Memory for Host $h$. The remaining memory, $R_{M}(h)$, can be calculated by

$$
R_{M}(h)=M_{h}-\sum_{v_{h}} M\left(v_{h}\right)-\alpha_{M}\left[\sum_{v_{h}, v_{h}^{\prime}, v_{h} \neq v_{h}^{\prime}} B\left(v_{h}, v_{h}^{\prime}\right)\right]
$$


Let $w_{M}(h)$ stand for the unit cost of consuming the memory resource of $h$. We model $w_{M}(h)$ to be inversely proportional to the remaining memory with constant of proportionality $W_{M}$. Define the memory cost of $h$ as $S_{M}(h)$. We have

$$
S_{M}(h)=\sum_{v_{h}} M(v) w_{M}(h)=\sum_{v_{h}} \frac{M\left(v_{h}\right) W_{M}}{R_{M}(h)+\delta},
$$

where $\delta$ is a small positive number to avoid dividing by zero.

\subsubsection{Cloud Costs}

For a mobile edge application with SFC where no host and link combination would be able to meet the requirements, the remote cloud can become an option. For the simplicity of our discussion, we give constant unit costs of CPU, memory, and bandwidth as $w_{C}(c), w_{M}(c)$ and $w_{B}(c)$.

Define the CPU, memory and bandwidth costs as $S_{C}(c), S_{M}(c)$, and $S_{B}(c)$. The total cost for deploying VMs on the cloud is denoted by $S(c)$. Compared to VM deployments on hosts on the mobile edge, apparently, deployment VMs on the cloud has significantly higher bandwidth cost. The total bandwidth from the mobile edge to the cloud is defined by $B_{c}$, which will be the bottleneck of cloud-based VM deployments if more VMs are placed on the cloud. The total cloud cost, denoted by $S(c)$, is then

$$
\begin{aligned}
S(c) & =S_{C}(c)+S_{M}(c)+S_{B}(c) \\
& =\sum_{v_{c}} C(v) w_{C}(c)+M(v) w_{M}(c)+B(v) w_{B}(c) .
\end{aligned}
$$




\subsubsection{Stochastic Programming}

Stochastic programming is an approach for modeling optimization problems that involve uncertainty. It provides a solution by eliminating uncertainty and characterizing it using probability distributions. There are in general two types of stochastic programming: probabilistic constraints and recourse problems. In probabilistic constraints, the optimization problems are deterministic with some constraints containing probability distribution functions. In recourse problems, the objectives are to optimize the results in average, which typically has two stages, where we make some decisions in the first stage and we make further decisions in the second stage to avoid the constraints of the problem becoming infeasible after seeing a realization of the stochastic elements. In this dissertation, our stochastic programming problems fall in the first type in the sense that some of constraints contain probability functions.

\subsubsection{Stochastic Programming Formulation}

The problem is formulated as a stochastic programming optimization as $P_{V}$ and $P_{H}$ used for minimum availability constraints are probability density functions of the random variables. Therefore, the stochastic programming we present is of type probabilistic constraints 108,109 .

Regarding its objective, the owner of the mobile edge application aims to minimize the cost when operating MEC services. As discussed in Sections 4.2.3 and 4.2.4, we

provide three sets of costs, which are $S_{B}\left(e_{i j}\right), S_{C}(h)$ and $S_{M}(h)$. The optimization is 
to minimize three types of costs over all hosts and their links.

$$
\begin{aligned}
& \text { Minimize } S(c)+\sum_{h} S_{C}(h)+\sum_{h} S_{M}(h)+\sum_{e_{i j}, i \neq j} S_{B}\left(e_{i j}\right) \\
& =\sum_{v_{c}} C(v) w_{C}(c)+M(v) w_{M}(c)+B(v) w_{B}(c) \\
& +\sum_{v_{h}} \frac{\left[C_{v_{h}}+\gamma_{C}\left(N_{V}-1\right)\right] W_{C}}{R_{C}(h)+\delta} \\
& +\sum_{v_{h}} \frac{\left[M_{v_{h}}+\gamma_{M}\left(N_{V}-1\right)\right] W_{M}}{R_{M}(h)+\delta} \\
& +\sum_{e_{i j}, i \neq j} \frac{B_{V}^{2}\left(e_{i j}\right) W_{B}\left[\sum_{v_{h_{i}}, v_{h_{j}}, h_{i} \neq h_{j}} L\left(v_{h_{i}}, v_{h_{j}}\right)\right]}{B\left(e_{i j}\right)\left[B\left(e_{i j}\right)-B_{V}\left(e_{i j}\right)\right] a_{i j}+\delta} \\
& \text { w.r.t. } \quad x_{v h} \\
& \text { s.t. } \quad B\left(e_{i j}\right) \geq \sum_{v_{h_{i}}, v_{h_{j}}, h_{i} \neq h_{j}} B\left(v_{h_{i}}, v_{h_{j}}\right) \\
& \sum_{v_{c}, v_{h}} B\left(v_{c}, v_{h}\right) \leq B_{c} \\
& C_{h} \geq \sum_{v_{h}}\left[C_{v_{h}}+\gamma_{C}\left(N_{V}-1\right)\right] \\
& +\alpha_{C} \sum_{v_{h}, v_{h}^{\prime}, v_{h} \neq v_{h}^{\prime}} B\left(v_{h}, v_{h}^{\prime}\right)
\end{aligned}
$$




$$
\begin{aligned}
& M_{h} \geq \sum_{v_{h}}\left[M_{v_{h}}+\gamma_{M}\left(N_{V}-1\right)\right] \\
& \quad+\alpha_{M} \sum_{v_{h}, v_{h}^{\prime}, v_{h} \neq v_{h}^{\prime}} B\left(v_{h}, v_{h}^{\prime}\right) \\
& \sum_{\bar{a}, \hat{a}=y}^{N_{V}} p_{\bar{a}} \geq 1-\eta
\end{aligned}
$$

\section{Remarks}

* Function (4.17) is the objective function. It targets to minimize the total cost. The host placement policy tends to find a sweet point minimizing the cost by using less hosts, while not exhausting them. The decision variable is $x_{v h_{m}}$ which indicates if $v$ is deployed on $h_{m}$.

* Constraint (4.18) is the link bandwidth capacity bounds between each two hosts. Traffic transmitted between any two hosts $h_{i}$ and $h_{j}$ must not exceed the corresponding bandwidth capacity $B\left(e_{i j}\right)$.

* Constraint (4.19) is the link bandwidth capacity bound from the mobile edge to the remote cloud. The total bandwidth consumption between the VMs at the edge and those on the cloud cannot exceed $B_{c}$, which is the bandwidth between the edge and the cloud.

* Constraints 4.20 and 4.21 are the CPU and memory capacity bounds for each host. The CPU and memory used by VMs coordinating with each other and by intra-host communications must not exceed $C_{h}$ and $M_{h}$.

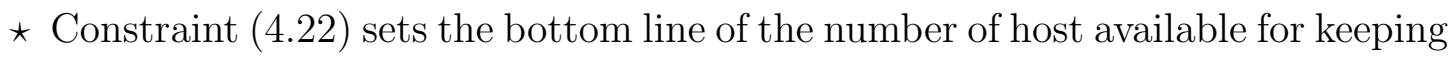


the mobile edge application available, i.e., the probability of at least $N_{m} \mathrm{VMs}$ in service must be greater than or equal to $1-\eta$. This constraint would lead to anti-affinity rules enforced among at least part of the VMs to ensure at least the required number of hosts are used.

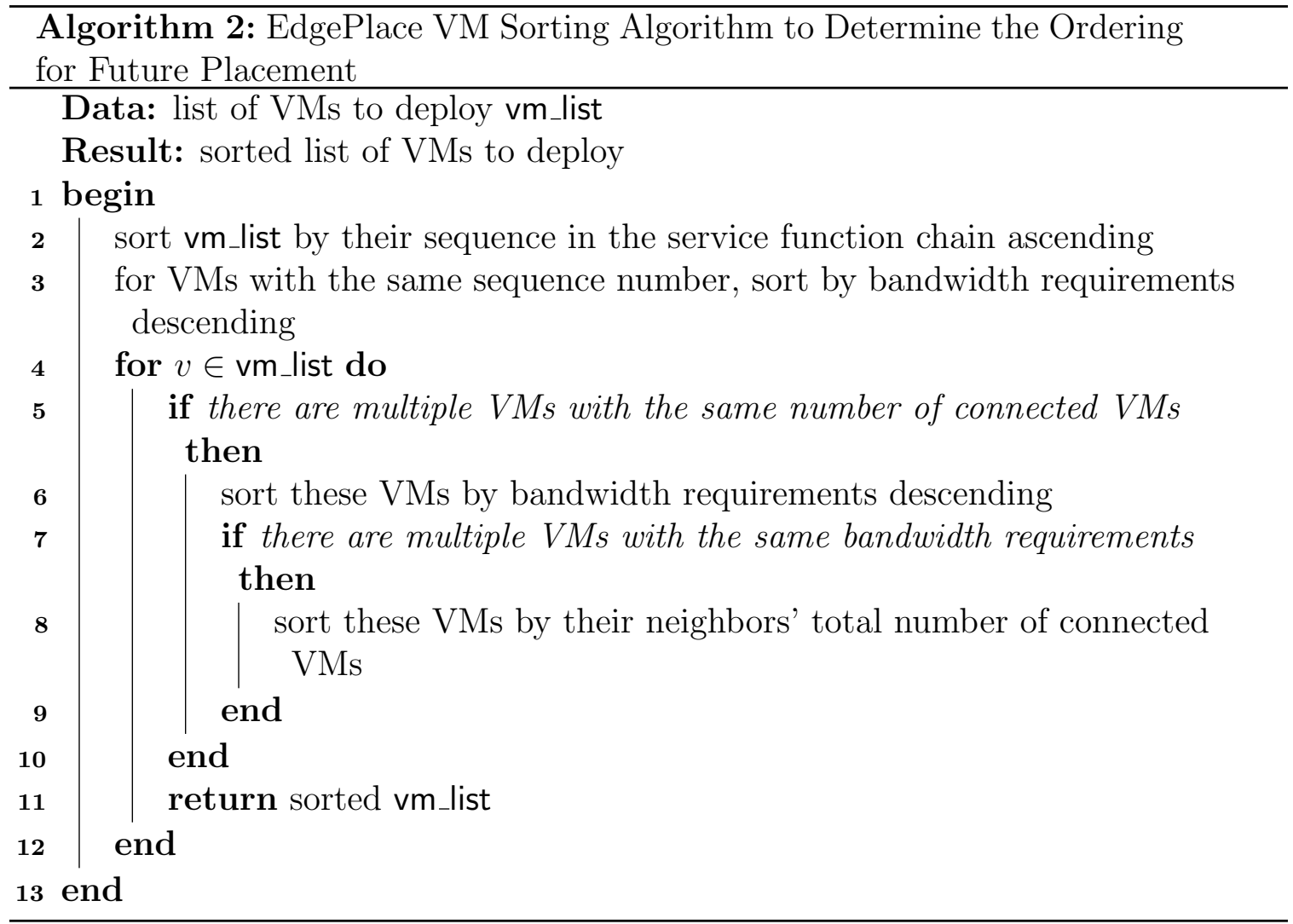

\subsubsection{Scalability and the EdgePlace Algorithm}

The formulation presented is one of the stochastic programming problems, where $P_{H}$ and $P_{V}$ are used in Constraint 4.22). Combining Equation (4.3), we found that Constraint 4.22 will contain exponential functions depending on the value of $a_{h}$. Therefore, the stochastic programming presented above is intractable. As the problem scales, it may not be computationally feasible to solve it. To apply our model 
to real-world scenarios, we develop a heuristic algorithm called EdgePlace to achieve suboptimal results by applying a hybrid strategy of best-fit and first-fit decreasing algorithm.

\section{Processing Order of VMs}

The EdgePlace algorithm will first process VMs with the most links to other VMs. These VMs tend to cause more inter-host traffic if migrated. Therefore, the algorithm tries to put VMs linked to each other on the same host. Even if the host is down, these VMs can be migrated together to another host without generating extra inter-host traffic. EdgePlace follows the ordering to sort VM as below, also shown in Algorithm 2 .

* Sort all VMs by the number of connected VMs (neighbors) descending.

* Sort VMs with the same number of connected VMs by their bandwidth requirement descending.

* Sort VMs with the same bandwidth requirement by the sum of connected VMs of their neighbors.

\section{Affinity first with service chain consideration}

As Algorithm 3 shows, when deploying a mobile edge application VM, all hosts are sorted based on their remaining resources as the first step. When the availability requirement is met, EdgePlace tries affinity first: the host with the most existing VMs deployed is attempted first to minimize inter-host traffic of the mobile edge application. If all constraints are satisfied, the host will be chosen to deploy the VM.

When a mobile edge application service is chained, traffic streams flowing through VMs will be directional. Unlike clustered VMs that can exchange data with each 
other, it doesn't save inter-host bandwidth if we put together two non-adjacent VMs in a service chain. In fact, it may cause traffic to go back and forth and worsen the situation. To reduce inter-host traffic by SFC, EdgePlace takes into consideration the sequence of VMs, and try to put adjacent VMs on the same host when the resource level permits. When moving placement decisions to another host, the algorithm will not consider previously-chosen hosts anymore, to avoid repeating traffic between hosts.

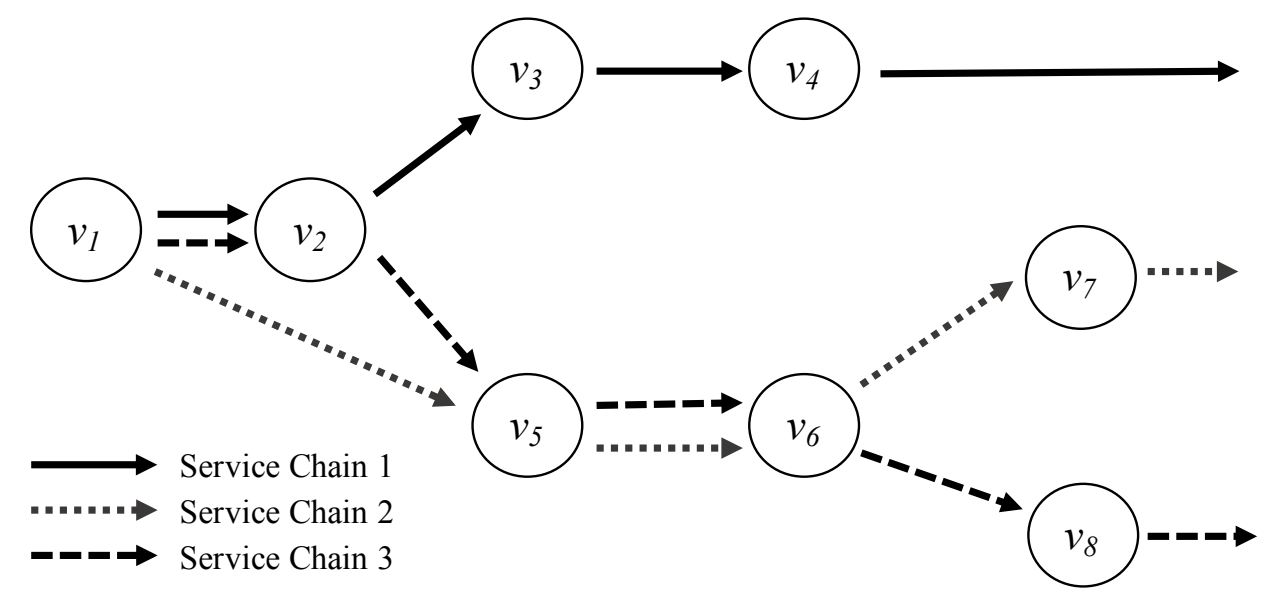

Figure 4.5: A service graph consisting of 3 service chains

Fig. 4.5 is an example of 3 service chains established on 8 VMs. Based on our algorithm, we fist determine the sequence of the VMs in the service chains. Note that if a VM is shared by more than one service chain, we take the smallest sequence. For instance, while $v_{5}$ is the third VM for Service Chain 3, it is also the second VM for Service Chain 2. Therefore, its sequence is 2. Based on the sequence of each VM, their placement will be determined by the algorithm accordingly. When the sequence of multiple VMs are the same, the priority of placement decision for those VMs will then be sorted by the bandwidth consumption. 


\section{Anti-affinity by bandwidth cost}

Otherwise, EdgePlace will try the host with the lowest link bandwidth costs given the VM to be deployed would cause inter-host traffic. At each step to calculate the potential inter-host bandwidth cost, the algorithm will only calculate the added bandwidth cost to VMs already deployed. The VMs yet to be deployed will not be considered until the algorithm reaches iterations to process them. The step will be repeated until it has tried all hosts or it has found the first valid host to deploy the VM. As one of the constraints, the availability change of the mobile edge application for the placement selection is tracked. To deploy all VMs, the worst time complexity of the algorithm is $O\left(N_{V}^{2} \log N_{H}\right)$, where sorting the hosts takes $O\left(\log N_{H}\right)$ and iterating all VMs on all hosts takes $O\left(N_{V}^{2}\right)$.

\subsection{Numerical Results}

In this section, we illustrate the numerical results of availability changes with different scenarios of mobile edge application deployments. Our simulations are done via the placement algorithm we have implemented using Java according to Algorithm 3.

\subsubsection{Assumptions}

To clearly demonstrate the focused trends, the following assumptions are made to simplify the modeling of the problem without losing generality. We first discuss the placement selection of the same mobile edge application with elasticity. This means variable numbers of VMs can be deployed for the application, but all VMs are of the same type and require the same amount of resources.

1. The unit costs of the CPU and memory across all hosts are the same. So are costs of network bandwidth across all links among hosts. 


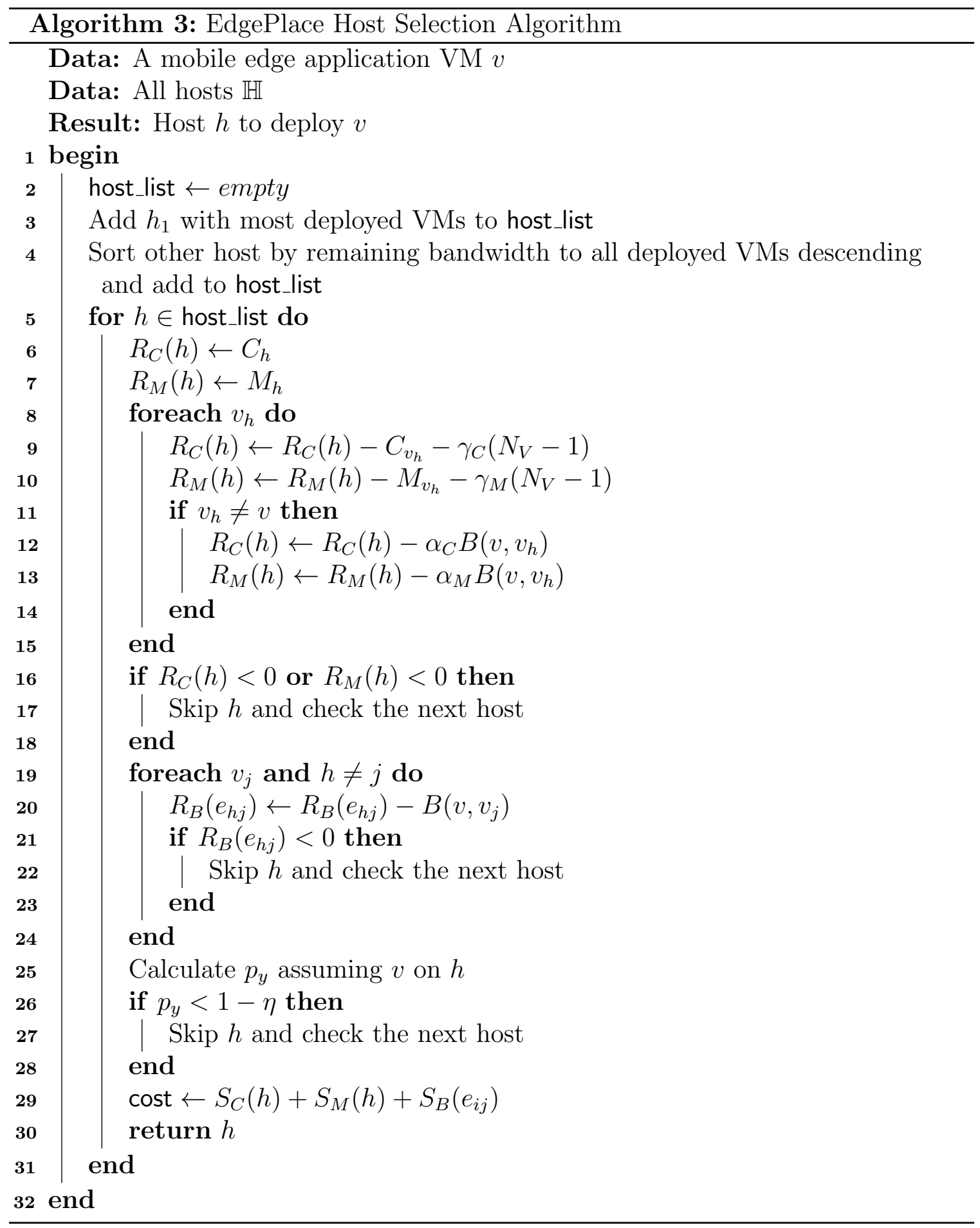


2. One mobile edge application includes the same type of VMs with the same CPU, memory and network bandwidth requirements.

3. A request from the user will be processed by one VM, while the VM may communicate with other VMs to exchange information.

\subsubsection{Parameters}

With the assumptions above, we choose parameters for our placement model to evaluate the performance and the facts under different circumstances.

\section{Hardware Requirement Profile}

The hardware requirement profile of a mobile edge application describe its resource needs. In this dissertation, it refers to the number of vCPUs, amount of memory and bandwidth. We define 10 types of hardware requirement profiles from F1 to F10, each with its unique requirements for the three resources.

Table 4.1: Pre-defined hardware requirement profiles for simulation.

\begin{tabular}{lllll}
\hline Name & $C_{v}$ & $M_{v}$ & $B_{v}$ & Max Latency $(\mathbf{m s})$ \\
\hline F1 & 1 vCPU & $1024 \mathrm{MB}$ & $1 \mathrm{Gbps}$ & $1000 \mathrm{~ms}$ \\
F2 & 2 vCPUs & $2048 \mathrm{MB}$ & $2 \mathrm{Gbps}$ & $100 \mathrm{~ms}$ \\
F3 & 2 vCPUs & $2048 \mathrm{MB}$ & $2 \mathrm{Gbps}$ & $1000 \mathrm{~ms}$ \\
F4 & 2 vCPUs & $2048 \mathrm{MB}$ & $2 \mathrm{Gbps}$ & $10000 \mathrm{~ms}$ \\
F5 & 4 vCPUs & $4096 \mathrm{MB}$ & $4 \mathrm{Gbps}$ & $100 \mathrm{~ms}$ \\
F6 & 4 vCPUs & $4096 \mathrm{MB}$ & $4 \mathrm{Gbps}$ & $1000 \mathrm{~ms}$ \\
F7 & 4 vCPUs & $4096 \mathrm{MB}$ & $4 \mathrm{Gbps}$ & $10000 \mathrm{~ms}$ \\
F8 & 8 vCPUs & $8192 \mathrm{MB}$ & $8 \mathrm{Gbps}$ & $100 \mathrm{~ms}$ \\
F9 & 8 vCPUs & $8192 \mathrm{MB}$ & $8 \mathrm{Gbps}$ & $1000 \mathrm{~ms}$ \\
F10 & 8 vCPUs & $8192 \mathrm{MB}$ & $8 \mathrm{Gbps}$ & $10000 \mathrm{~ms}$ \\
\hline
\end{tabular}

We choose certain parameters in our model as constants, while others as variables, shown as Table 4.2, where values are specified for constants, and variables are marked as var. For the constants, it is reasonable to assume that they are fixed for a specific 
mobile edge application deployment scenario. For the variables, they can change because scaling and SLA may change due to the nature of MEC. We will further discuss the variables in different sets of experiments.

Table 4.2: Parameters for availability-aware host selection

\begin{tabular}{ll|ll}
\hline Parameter & Value & Parameter & Value \\
\hline$C_{v}$ & 2 vCPUs & $M_{v}$ & $2048 \mathrm{MB}$ \\
$\alpha_{C}$ & $10 \%$ & $\alpha_{M}$ & $10 \%$ \\
$M_{h}$ & $102400 \mathrm{MB}$ & $C_{h}$ & $100 \mathrm{vCPUs}$ \\
$B\left(v, v^{\prime}\right)$ & $20 \mathrm{Mb}$ & $\gamma_{C}$ & $10 \%$ \\
$\gamma_{M}$ & $0.1 \%$ & $B\left(e_{i j}\right)$ & $10000 \mathrm{Mb}$ \\
$P_{V}$ & $90 \%$ & $P_{H}$ & $90 \%$ \\
$T_{h}$ & $5 \mathrm{~ms}$ & $T_{c}$ & $100 \mathrm{~ms}$ \\
$k_{t h}$ & 1.1 & $k_{t c}$ & 1.5 \\
$\delta$ & 0.1 & $W_{M}$ & var \\
$W_{C}$ & var & $\eta$ & var \\
$W_{B}$ & var & $N_{H}$ & var \\
$N_{V}$ & var & & var \\
\hline
\end{tabular}

\subsubsection{Availability Impact from Number of Instances and Hosts}

We first discuss the mobile application availability trends with various numbers of instances deployed on certain numbers of hosts. The discussion assumes constants $W_{C}=W_{M}=W_{B}=5$, and $y=3$. Also, $\eta$ is set to be 1 such that no minimum availability is required. This will help observe the trends of availability changes.

Fig. 4.6 demonstrates availability changes using the EdgePlace Algorithm with the number of instances deployed ranging from 4 to 16, and the number of hosts from 1 to 4 . Compared to the availability changes with the same parameters but using the stochastic programming proposed in Section 4.2, shown by Figure 4.7, there are only slight drops of availability with the heuristic algorithm, proving the effectiveness of the algorithm. From the results, we can clearly see that increasing the number of backup VMs can improve the availability of the mobile edge application. However, 

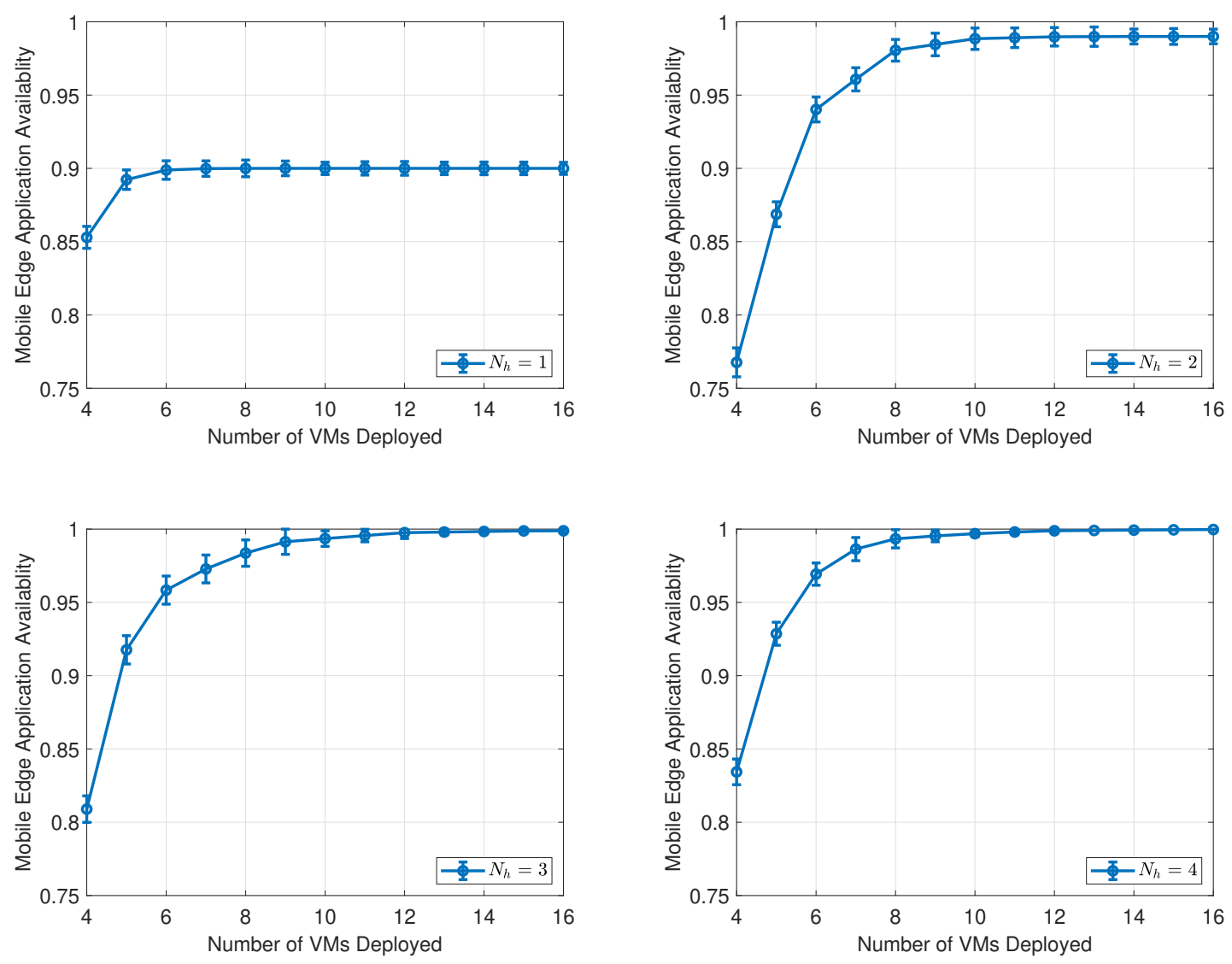

Figure 4.6: Availability of the mobile edge application with different numbers of VMs deployed on various numbers of mobile edge hosts using the EdgePlace algorithm. Confidence level is $95 \%$

there is a ceiling of the availability by only increasing the number of VMs due to host availability. When $N_{H}=1$, that maximum availability cannot exceed 0.9 . To meet the minimum availability, there must be enough hosts to keep the theoretical availability above $1-\eta$.

Furthermore, Fig. 4.8 shows the negative impact from $y$, the minimum number of VMs required by the mobile edge application. With the $N_{V}=10$ as a constant, having a larger $y$ would lower the availability of the application. Considering to increase the number of VMs as well as hosts may be necessary when demand is high. 


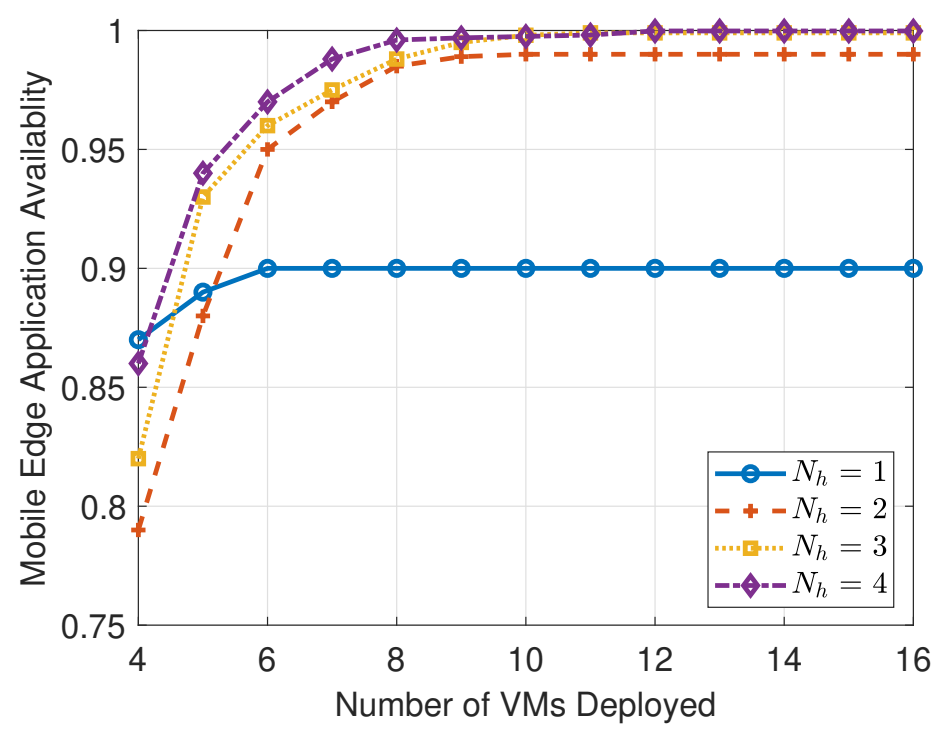

Figure 4.7: Availability of the mobile edge application with different numbers of VMs deployed on various numbers of mobile edge hosts using the stochastic model

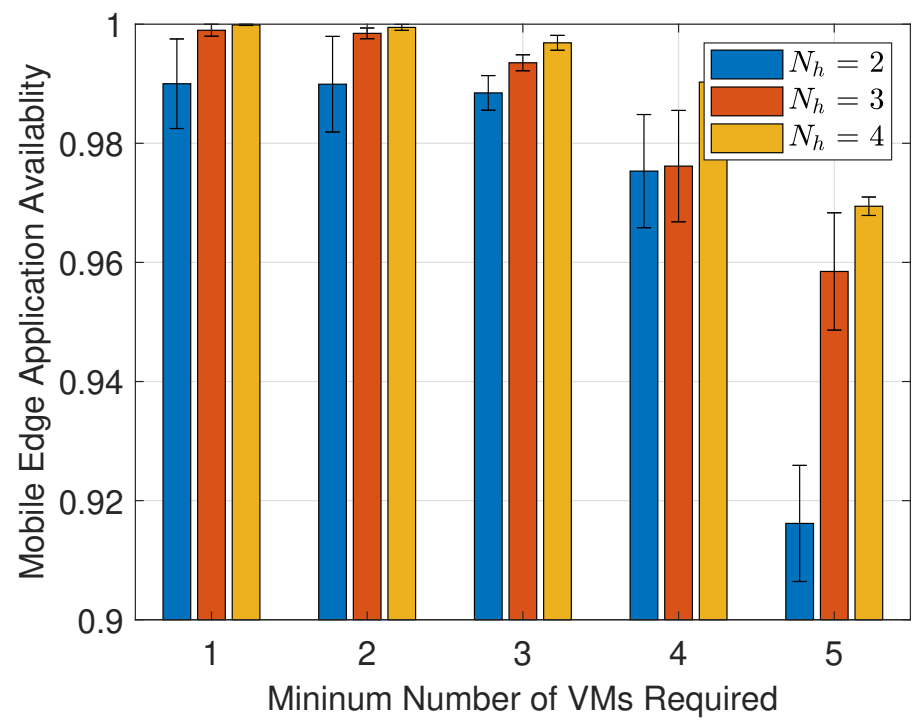

Figure 4.8: Availability of the mobile edge application with varying minimum number of VMs required and different numbers of mobile edge hosts. Confidence level is $95 \%$ 


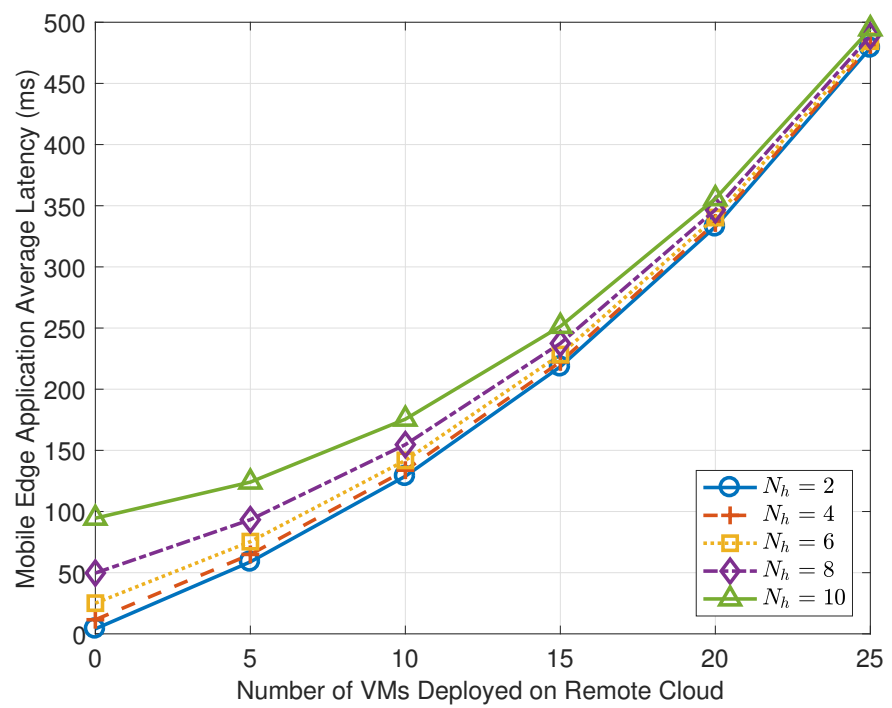

Figure 4.9: Average latency of the mobile edge application with different numbers of VMs deployed on remote cloud and various numbers of hosts

\subsubsection{Latency}

Assume the latency of a link between each two MEC hosts to be $T_{h}$, while the link between a MEC host and the remote cloud to be $T_{c}$. As more VMs deployed for one mobile edge application, traffic increases among them to keep them in sync and to coordinate with each other. We set $T_{h}=6 \mathrm{~ms}$, and $T_{c}=200 \mathrm{~ms}$. The latencies caused by inter-host traffic and host-cloud traffic are shown in Fig. 4.9. The results have demonstrated an increase of latency as the number of hosts grows for deploying the mobile edge application. Depending on the definition of the application and its maximum latency allowed, we can determine the maximum number of VMs offloaded to the cloud under different numbers of mobile edge hosts. 

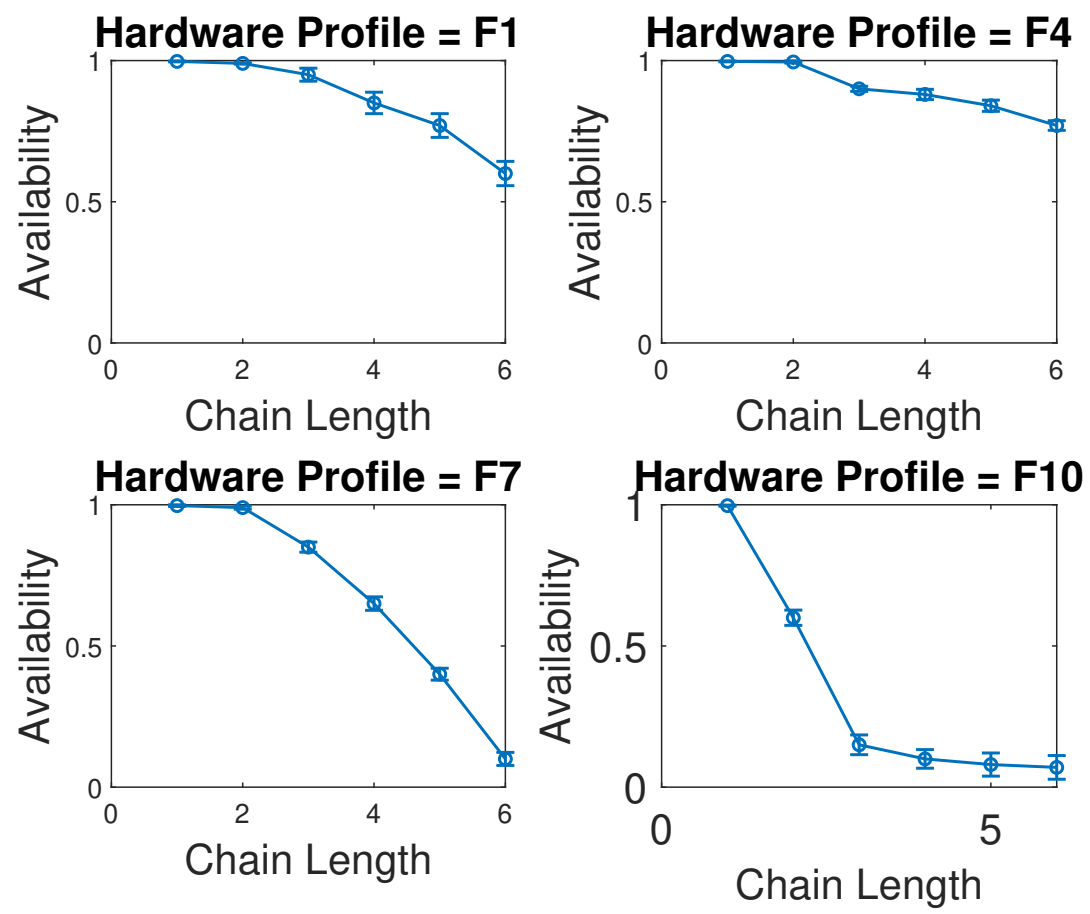

Figure 4.10: Availability of an application consisting of $6 \mathrm{VMs}$ with $95 \%$ confidence level and four hardware requirement profiles (F1, F4, F7, and F10) 


\subsubsection{Availability Impact from SFC}

The portability of a mobile edge application due to SFC can affect the application availability. We compare the availability among mobile edge applications with different levels of SFC. We first define a mobile edge application consisting of $6 \mathrm{VMs}$ with different level of chaining, starting from no chaining, to the first certain number of VMs chained, and to all 6 VMs chained. In general, SFC will bring negative impact to the availability, because it sets more constraints when placing VMs, making it more difficult to find valid place for hosting the VMs. Four pre-defined hardware requirement profiles from Table 4.1, namely F1, F4, F7, and F10, are chosen for creating VMs for the target mobile edge application. Fig. 4.10 shows availability changing trends under 4 hardware requirement profiles. From the results, we find the availability plunges faster when VMs have higher hardware requirements. This is due to the increasing challenge to find proper host combinations to deploy the application with SFC.

\subsubsection{Distribution of VMs with Different Bandwidth Costs}

The impact of $W_{B}$, which can be considered as the network bandwidth price index, is evaluated in this section. We set up $N_{H}=10, N_{V}=20, y=2, W_{C}=W_{M}=$ 1 , and $\eta=0.3 \%$. We choose various values of $W_{B}$ and the results of VM placements under each value of $W_{B}$ on 10 hosts from $H 01$ to $H 10$, as shown in Fig. 4.11. From the results, it can be learned that higher price of the network bandwidth will cause more concentrated placement of the VMs. When the host networks become congested, EdgePlace would put VMs on less hosts to limit inter-host traffic, with the trade-off lowering the availability of the mobile edge application. When the hosts used are reduced to 3 with a 9-9-2 VM distribution, the availability is close to the bottom line of $99.73 \%$. If $W_{B}$ continues to hike, choosing a 10-9-1 VM distribution would achieve 
the lowest cost, but would violate the minimum availability requirement. Therefore, the 9-9-2 VM distribution is the best placement decision when $W_{B} \geq 45$.

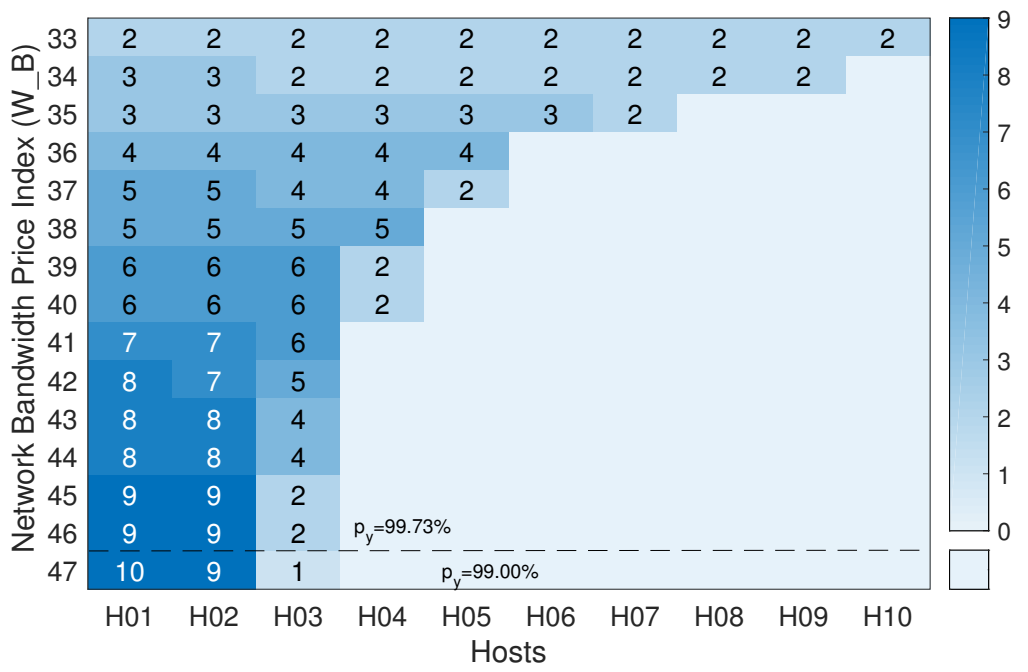

Figure 4.11: Placement distribution of $20 \mathrm{VMs}$ across 10 hosts with $N_{H}=10, N_{V}=$ $20, y=2, W_{C}=W_{M}=1$, and $\eta=0.3 \%$

\subsection{Chapter Summary}

In this chapter, we have formulated a mobile edge application placement problem and presented a heuristic algorithm called EdgePlace. Our work promotes reducing the cost by introducing extra mobile edge hosts and balancing the workload of the hosts. Rather than overloaded, expensive hosts, VMs are deployed on less busy hosts to achieve lower cost. Meanwhile, the availability of the mobile edge application has also been profoundly improved as a result of leveraging multiple hosts. Our future work is to consider mobile edge applications with combinations of different NFs and with service chaining. 


\section{Chapter 5}

\section{CPE Resource Sharing At The Edge}

\subsection{Introduction}

Customer-premises equipment or customer-provided equipment (CPE) is any terminal and associated equipment located at a subscriber's premises and connected with a carrier's telecommunication circuit at the demarcation point. In the wake of cloud computing and Network Function Virtualization (NFV) [12 [13], Service Providers (SPs) leverage virtual Customer Premises Equipment (vCPE) as Virtual Network Function (VNF) instances on top of generic physical Customer-Premises Equipment (pCPE), in search of rebuilding a dynamic revenue stream [110].

There can be enough resources for pCPE to deploy VNFs locally [14], while pCPE can also coordinate with the cloud if VNF scale-out is needed to accommodate heavier usage. However, large-number deployments of Internet-of-Things (IoT) devices bring challenges to VNFs running in a centralized cloud, as the network traffic load would be drastically increased by transmitting data between the core and the edge of the network. Such traffic overhead can become unacceptable with excessive data transmission, causing high processing delay or even service outage due to the congestion of the network. Meanwhile, high usage of the cloud networks would jack up the price 
per usage, resulting in higher-than-expected operating expense (OPEX).

Recent research has been aware of the explosive growth of devices in the edge of the networks. The concept of fog computing [24] and edge computing [5] were proposed to move the initial handling of raw data to the edge for IoT devices. Although the fog can mitigate the load of the core network, the power of the Customer Edge (CE), namely the computational capabilities of $\mathrm{CPE}$, is buried. While a single pCPE node has limited resources and typically serves a designated location, the aggregated computing capabilities of pCPE nodes across the edge of a network can be powerful: pCPE nodes have time-varying resource usage that does not always reach full workloads. For instance, the home gateways typically have significantly lower usage in business hours as their users leave for work, while office gateways become idle in after hours. If the spare resources of pCPE can be shared within the network edge, VNFs will be able to roam around the edge. Both SP and users will benefit from the considerable capabilities of the sharable resources. Meanwhile, the VNFs deployed on the pCPE nodes keep most traffic within the edge and reduce the traffic to the core network.

However, CPE resource sharing faces challenges. including to quantify the benefit from sharing spare resources of pCPE nodes, maintaining required service availability, and to motivate users to participate in resource sharing. In this chapter, we propose an architecture to allow sharing resources of $\mathrm{pCPE}$ within the network edge, namely IoT-B\&B. We discuss the scenario that SP deploys VNFs, which are vCPE instances, to both the cloud and the available pCPE nodes participating in the resource sharing program. As Figure 5.1 shows, when a sharable pCPE node is not actively used by its owner, it will be treated as a "bed-and-breakfast" place for vCPE instances to "stay". SP will have the permission to utilize free resources through the resource manager to deploy VNFs of other users from the same edge network. The following contributions are made for enabling crowdsourcing at the network edge by utilizing 


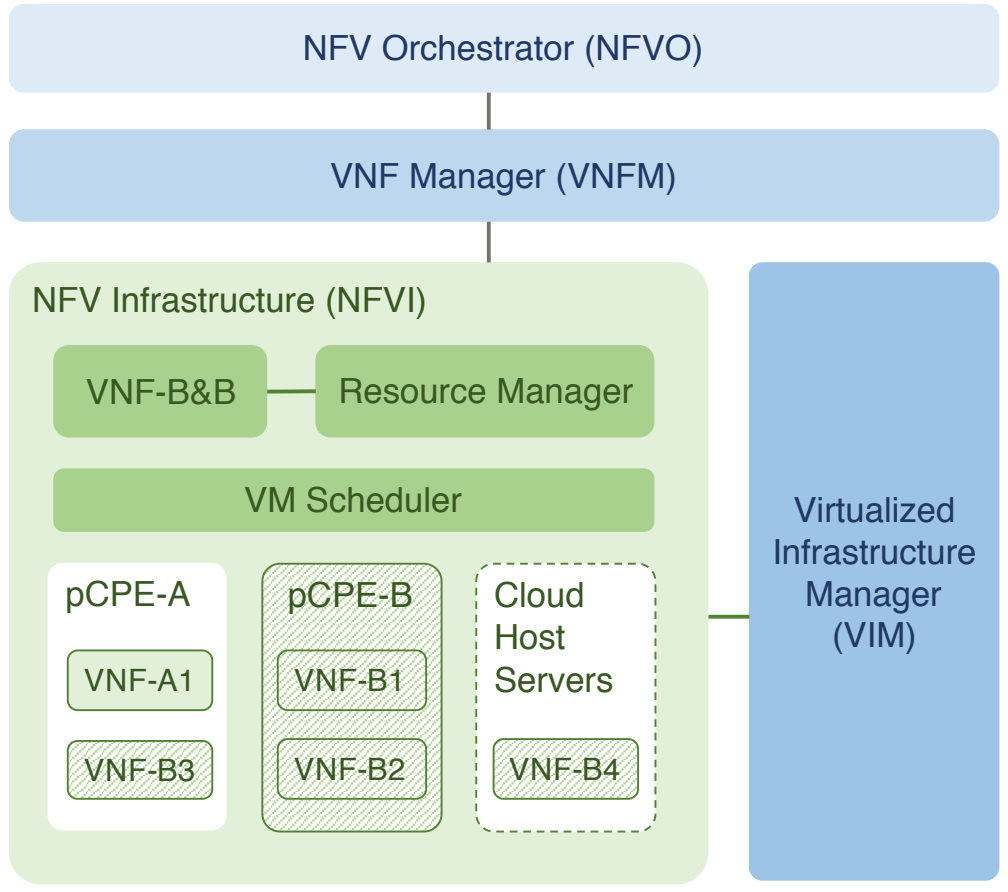

Figure 5.1: The system architecture extended from ETSI NFV architecture

resources of pCPE nodes:

(i) We propose an architecture extended from ETSI NFV architecture and interfaces 75$]$ to support resource sharing of pCPE nodes. The pCPE nodes at the network edge are treated as compute hosts which can have VNF instances deployed. They are grouped together and abstracted into the NFV Infrastructure (NFVI) layer. A resource manager is embedded in the NFVI and can leverage placement algorithms to make placement decisions.

(ii) A model is presented to evaluate the cost of assigning a VNF instance to a pCPE node and to the remote cloud. Multiple factors are considered to determine the cost, including remaining resources, network transmission delay, and availability requirements.

(iii) A placement algorithm called "IoT-B\&B Algorithm" is also presented to for assign vCPE instances to pCPE nodes with the goal to find a cost-efficient 
pCPE node for each VNF.

(iv) We implement a system with the IoT-B\&B architecture with steps to setup the NFVI and the system's life cycle. Numerical results are shown to demonstrate the placement algorithm's effectiveness.

We divide the contents into the following sections. Section 5.2 formulates the problem. Section 5.3 proposes the IoT-B\&B Algorithm based on the problem formulation. Then Section 5.4 is presented, covering the actual implementation of the system, followed by the numerical results in Section 5.5. Section 5.6 concludes this chapter and lists future work items.

\subsection{Problem Formulation}

In this section, we formulate the problem by modeling the resource properties and constraints of the network edge. The resource types we discuss are limited to CPU, memory and network bandwidth. We believe these three types of the resources are most representative for cost estimation and optimization. Adding consideration of more resource types will not necessarily change the optimization model. Then the properties of the VNF instances are defined and annotated. Note that the terms "VNF", "VNF instance" and "vCPE instance" in this dissertation are inter-changeable. The definitions of symbols used can be found in List of Symbols.

\subsubsection{Connected pCPE at Network Edge}

We discuss one particular network edge, which includes all pCPE nodes under it. A network edge is defined as the networks connecting all pCPE nodes under it. We model a network edge to have a topology such that each pCPE node within it can communicate with another. An example with two network edges can be found in 


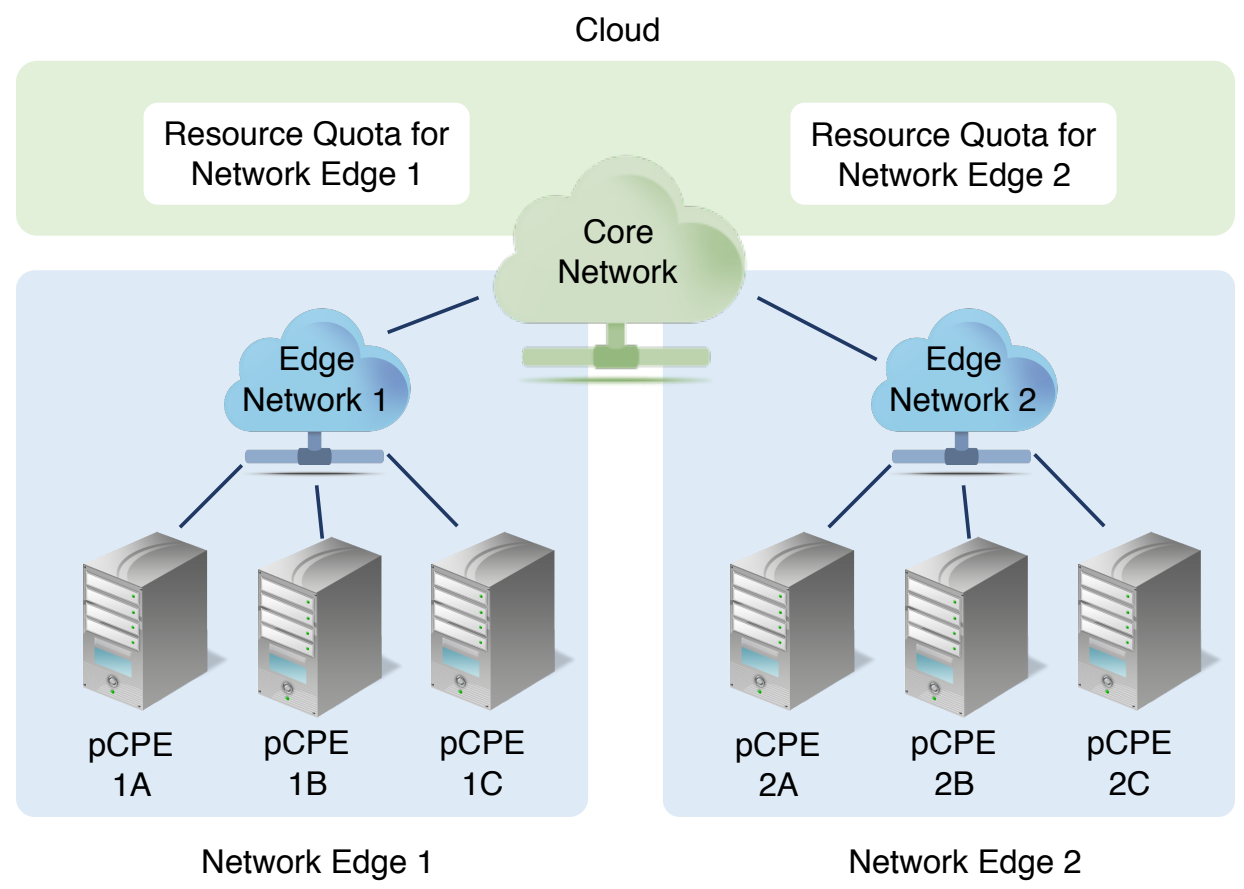

Figure 5.2: Topologies at network edges

Figure 5.2. The nodes of pCPE 1A, 1B, 1C group as one network edge connected to each other via Edge Network 1, while the other one consists of the nodes $2 \mathrm{~A}, 2 \mathrm{~B}, 2 \mathrm{C}$, connected by Edge Network 2.

Based on the definition above, the pCPE nodes and their links at the network edge can be modeled as a directed graph $G=(V, L)$. Set $V$ represents all pCPE nodes st the network edge, while $L$ is the set of all connected links from one vCPE node to another. A pCPE node in $V$ is denoted by $v$, there is $v \in V$. Define the total number of pCPE nodes to be $n_{V}$. Let $v_{i}$ be a specific pCPE node in $V$, such that

$$
v_{i} \in V, \forall i \in\left[1 . . n_{V}\right]
$$

Since all pCPE nodes are connected to each other, $G$ is strongly connected. For any data transmitted from one node $v_{i}$ to another node $v_{j}$ in $V$, there exists a link $l_{i j}$, 
such that

$$
l_{i j} \in L, \forall i, j \in\left[1 . . n_{V}\right], i \neq j .
$$

The network edge is connected to the core network via a logical link, denoted by $l_{c}$. The capacity of $l_{c}$ is limited due to budget reasons: the network edge has a certain bandwidth quota. The total bandwidth to the cloud must be kept within the quota. In reality, $l_{c}$ can be a group of links connecting the remote cloud.

\subsubsection{VNF Types and Resource Requirement Profiles}

The network functions serving the IoT networks have been encapsulated into various types of VNFs. Each type provides a user with specific service. When the demand increases for a certain type of VNF to a certain level that it exceeds the maximum capacity of current VNF instances, the VNF scales out by increasing the number of Virtual Machine (VM) instances, so that more requests can be processed at the same time. Depending on the purpose, different types of VNF have different resource requirements. The term "flavor" is used to describe the resource requirements profile of an instance of the VNF, including the number of vCPUs, the amount of memory, the size of the disk, and so on. We use $f$ to define a VNF instance.

Assign $a$ to identify a specific type of network function and $n_{a}$ to be the number of network function types. A VNF instance with type " $a$ " can then be represented by $f(a)$. The CPU, memory, and bandwidth requirements of $f(a)$ are then denoted by $U(f(a)), M(f(a))$, and $B(f(a))$. 


\subsubsection{The User of a VNF instance}

Another property of a VNF instance is the user who owns and uses it. Suppose each pCPE node has one unique owner. This is a valid assumption as the actual device on customer premise is typically not shared and belongs to the entity who pays for the service. For a pCPE node $v$, its owner $u$ uses a set of VNF instances to satisfy its needs, regardless of where the instances are deployed. When referring to a specific node $v_{i}, i \in\left[1 . . n_{V}\right]$, we represent the node's owner by $u_{i}$, where $i \in\left[1 . . n_{V}\right]$.

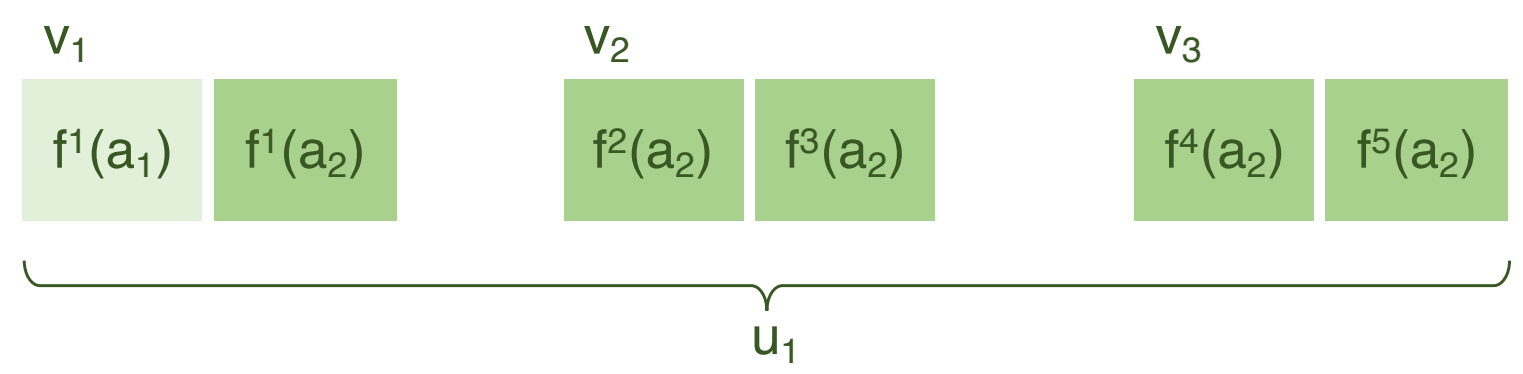

Figure 5.3: An example of VNF instances grouped by $u_{1}$

Figure 5.3 illustrates an example of VNF instances grouped by the user $u_{1}$. There is 1 instance of $f\left(a_{1}\right)$ and 5 instances of $f\left(a_{1}\right)$. The only instance of $f\left(a_{1}\right)$, denoted by $f^{1}\left(a_{1}\right)$, is on $v_{1}$, along with $f^{1}\left(a_{2}\right)$. Instances $f^{2}\left(a_{2}\right)$ and $f^{3}\left(a_{2}\right)$ are on $v_{2}$. Instances $f^{4}\left(a_{2}\right)$ and $f^{5}\left(a_{2}\right)$ are on $v_{3}$. The numbers and types of VNF instances grouped by their users are determined by user activities and can change dynamically according to user demands.

Based on the user of VNF instances, the annotation of $f$ can be extended from $f(a)$ to $f(a, u)$, where $u$ is the user who owns and uses $f$.

\subsubsection{Places to Deploy VNF instances}

Placement decisions are made based on the flavors, which are the resource requirement profiles, of VNF instances and the resource capacities of pCPE nodes. A VNF instance 
of a certain user $u_{i}$, denoted by $f\left(a, u_{i}\right)$, can be deployed at either of the locations below.

\section{$\mathrm{B} \& \mathrm{~B}$ deployment}

For a VNF instance $f\left(a, u_{i}\right)$, any pCPE node within the same network edge can be considered as a candidate place to deploy, also known as B\&B deployments. A B\&B deployment is performed when $f\left(a, u_{i}\right)$ is deployed on a pCPE node $v_{j}$. For a B\&B deployment of $f\left(a, u_{i}\right)$, its notation can be extended to $f\left(a, u_{i}, v_{j}\right)$, where $v_{j}$ is the place to deploy $f$. We use the set $F_{v}$ to define all VNF instances deployed on the pCPE node $v$.

Particularly, when there are enough resources on the pCPE node $v_{i}$ of $u_{i}, f\left(a, u_{i}\right)$ can be deployed on $v_{i}$ locally. For a local deployment of $f\left(a, u_{i}\right)$, its notation becomes $f\left(a, u_{i}, v_{i}\right)$, where $v_{i}$ is the place to deploy $f$.

\section{Cloud deployment}

Besides B\&B deployments, the remote cloud can be chosen as an alternative place to deploy VNF instances. For a cloud deployment of $f\left(a, u_{i}\right)$, its notation can be extended to $f\left(a, u_{i}, c\right)$, where $c$ is the place to deploy $f$, and $c$ stands for the remote cloud location. We use the set $F_{c}$ to define all VNF instances deployed on the cloud.

\subsubsection{Factors to Impact Placement Decisions}

The following factors will impact the placement decisions.

\section{pCPE Resource Capacity}

Every pCPE node $v$ has its own resource capacity to host a limited number of VNF instances. As a compute node, the resources for VNF instances are: virtual CPUs 
vCPU Capacity $=20$

\begin{tabular}{cccccc|c}
$f^{1}\left(a_{1}\right)$ & $f^{2}\left(a_{1}\right)$ & $f^{1}\left(a_{2}\right)$ & $f^{2}\left(a_{2}\right)$ & $f^{3}\left(a_{2}\right)$ & Free vCPU \\
2 & 2 & 4 & & 4 & 4 & \\
& & & & & & \\
$f^{1}\left(a_{1}\right)$ & $f^{2}\left(a_{1}\right)$ & $f^{1}\left(a_{2}\right)$ & $f^{2}\left(a_{2}\right)$ & $f^{3}\left(a_{2}\right)$ & Free Memory \\
$2 G B$ & $2 G B$ & $1 G B$ & $1 G B$ & $1 G B$ &
\end{tabular}

Memory Capacity $=10 \mathrm{~GB}$

Figure 5.4: Capacities of vCPUs and memory of a pCPE node

(vCPUs) and memory. Note that memory in discussion refers to physical RAM of host servers, which does not include virtual memory on disk. We assume that there is plenty of disk space on each pCPE node for any virtual instances deployed. Therefore, it is not in the scope of discussion. Let $U(v)$ denote the number of vCPUs $v$ can provide. Let $M(v)$ be the total amount of memory for VNF instances from $v$. Figure 5.4 provides an example of the resource capacity for a pCPE node with 20 vCPUs and 10 GB memory in total. There are currently 2 instances of VNF $f\left(a_{1}\right)$ and 3 instances of $f\left(a_{2}\right)$ deployed on it.

\section{Edge Network Transmission Delay}

Comparing with the core network transmission delay to be discussed in the next section, the transmission delay between pCPE nodes at the network edge can be ignored as the bandwidth between edge nodes is considered plenty and the transmission delay is small enough to be ignored in the discussion. 


\section{Core Network Transmission Delay}

The core network transmission delay of a VNF instance $f$ offloaded to the cloud is defined as the time consumed by offloading the VNF instance to the cloud and is denoted by $t(f)$, while $T_{\max }(f)$ is the maximum allowed network delay for a specific network function instance. The actual delay must not exceed this limit, or the requests would eventually overflow the buffer and cause malfunction of the VNF.

There are many factors that may affect the core network transmission delay. As stated in [111], the transmission delay to the cloud can be calculated by

$$
\text { Transmission delay }=\frac{\text { Message size }}{\text { Network bandwidth }} .
$$

For the same message, the less available bandwidth left from the network edge to the cloud, the longer the transmission delay will be. During peak hours, more VNF instances are requested by users concurrently across the network and would congest $l_{c}$. The severity of direct oversubscription is reflected by the residue bandwidth of the link from the edge switch to the cloud, denoted by $R\left(l_{c}\right)$, which is the link's total bandwidth $B\left(l_{c}\right)$ less the mean bandwidth usage for all remote VNF instances. The smaller residue bandwidth $R\left(l_{c}\right)$ there is, the bigger core network transmission delay $t\left(l_{c}\right)$ we should expect.

Because all network function instances offloaded to a remote cloud share the same link $l_{c}$ that connects the edge network to the remote cloud and the same cloud environment, we assume the core network transmission delay is the same for all offloaded network function instances to simplify the modeling process. Let $B\left(l_{c}\right)$ be the total bandwidth of $l_{c}$, and $t\left(l_{c}\right)$ be the transmission delay of $l_{c}$. VNF instances take up the bandwidth of $l_{c}$ to communicate with the pCPE nodes. The latency of the core network is therefore highly correlated to the bandwidth consumption of $l_{c}$. 
Let $F_{c}$ denote the set of VNF instances deployed in the remote cloud. $R\left(l_{c}\right)$ can be calculated by:

$$
R\left(l_{c}\right)=B\left(l_{c}\right)-\sum_{f \in F_{c}} B(f), \forall f \in F_{c}
$$

Even if $l_{c}$ is not congested, the remote cloud environment may be degraded by other sources. For example, overloaded VNFs from other users or applications of a shared cloud could affect other VNFs on the same host because of overcommitting. To better utilize the resources on a host, overcommitting is enabled by default 112. However, the performance could be jeopardized if some VNFs are taking up most resource [113]. We define the delay not directly caused by the network edge as a $T_{d}$, where the value of $T_{d}$ changes according to the load of the cloud environment.

Oversubscription will result in a higher core network delay $t\left(l_{c}\right)$. For all VNF instances offloaded to the cloud, there must be

$$
t\left(l_{c}\right) \leq T_{\max }(f), \forall f \in F_{c} .
$$

The equation above ensures the functionality of all VNFs offloaded to the cloud with the existence of $t\left(l_{c}\right)$. It draws a limit of how much VNF offloading can be done, since a oversubscribed cloud environment would increase $t\left(l_{c}\right)$. Based on the calculation of transmission delay, we further model $t\left(l_{c}\right)$ to be inversely proportional to $R\left(l_{c}\right)+b$, and proportional to $T_{d}$ with $b$ as a constant scoping the maximum core network delay when the bandwidth of $l_{c}$ is depleted:

$$
t\left(l_{c}\right)=\frac{T_{d}}{R\left(l_{c}\right)+b}, 0 \leq R\left(l_{c}\right) \leq B\left(l_{c}\right)
$$


Combining Equation (5.5) with Equation (5.6), we have

$$
\frac{T_{d}}{R\left(l_{c}\right)+b} \leq T_{\max }(f), \forall f \in F_{c} .
$$

When $T_{d}$ gets higher or $R\left(l_{c}\right)$ becomes lower, the value of $t\left(l_{c}\right)$ would exceed $T_{\text {max }}(f)$ of one or more offloaded VNF instances.

\subsubsection{Cost of Offloading to Edge Network}

By enabling the resource sharing of pCPE nodes across the network edge, SP benefits from extended containers hosting the VNFs. The costs of leveraging these resources include the following.

\section{Incentives Returned to End Users}

By encouraging the users to participate the resource sharing program and to consent to share, it is necessary to give incentives to the users based on the amount of resource shared. We denote the unit incentive of CPU, memory and bandwidth usage for pCPE node $v$ as $w_{U}(v), w_{M}(v)$ and $w_{B}(v)$, respectively. One exception is that when the pCPE node is hosting VNF instances used by the its own user, the incentives do not apply.

\section{Extra Redundancy}

Since the availability of the pCPE nodes is lower than the cloud, more standby VNF instances are needed. We define the redundancy factor $\gamma$ to be the mean number of standby VNF instances needed for one VNF instance on B\&B nodes. The redundancy factor is determined by the use case and shall be provided by the VNF operator.

Based on the two factors above, the cost of offloading a VNF instance $f$ to any of 
the pCPE node, denoted by $S(f, v)$, is calculated as below:

$$
S(f, v)=(1+\gamma)\left[w_{U}(v) U(f)+w_{M}(v) M(f)+w_{B}(v) B(f)\right] .
$$

\subsubsection{Cost of Offloading to Cloud}

As defined earlier, We use $c$ to represent the remote cloud in general to deploy VNF instances, to distinct from $\mathrm{B} \& \mathrm{~B}$ deployments. Although the resources in the cloud, especially in the public cloud, can be considered infinite [114] due to its elasticity, for a specific edge network, there are budgets for resources assigned to it. Therefore, resources in the cloud to an edge network are limited when we model them.

The total amounts of vCPUs, memory, and network bandwidth assigned to the edge network we discuss in the cloud are denoted by $U(c), M(c)$, and $B(c)$, respectively. The cost of offloading to the cloud depends on its usage. In general, the less resources left in the cloud for the edge network, the higher unit price our model gives, because the cloud needs to be available as an alternative place to host $\mathrm{vCPE}$ instances. Allowing the cloud resources to be drained too early will jeopardize the flexibility of placement and do harm to the service availability.

Let $w_{U}(c)$ stand for the unit cost for consuming the cloud's CPU resource. We model $w_{U}(c)$ to be inversely proportional to the cloud's remaining vCPUs with the constant of proportionality $W_{U}$. The remaining number of vCPUs is denoted by $R_{U}(c)$. The total cost of vCPUs for a VNF instance $f$ to be offloaded to the cloud, denoted by $S_{U}(f, c)$, is then:

$$
\begin{aligned}
S_{U}(f, c) & =w_{U}(c) U(f)=\frac{W_{U}}{R_{U}(c)+\delta} U(f) \\
& =\frac{W_{U} U(f)}{U(c)-\sum_{f^{\prime} \in F_{c}} U\left(f^{\prime}\right)+\delta} .
\end{aligned}
$$


In Equation (5.9), $\delta$ is a small positive number to avoid dividing by zero.

Also, let $w_{M}(c)$ represent the unit cost of the memory resource in the cloud. Like vCPUs, $w_{M}(c)$ is modeled to be inversely proportional to the cloud's residue memory with the constant of proportionality $W_{M}$. The residue memory resource of the cloud is denoted by $R_{M}(c)$. Similar to the induction of Equation (5.9), we have the total cost of cloud memory for a VNF instance $f$ denoted by $S_{M}(f, c)$, where $\delta$ is a small positive number to avoid dividing by zero:

$$
\begin{aligned}
S_{M}(f, c) & =w_{M}(c) M(f)=\frac{W_{M}}{R_{M}(c)+\delta} M(f) \\
& =\frac{W_{M} M(f)}{M(c)-\sum_{f^{\prime} \in F_{c}} M\left(f^{\prime}\right)+\delta} .
\end{aligned}
$$

Let $w_{B}(c)$ denote the unit cost of the remote cloud's network bandwidth. The variable $w_{B}(c)$ is defined to be proportional to the core network delay $t\left(l_{c}\right)$ with the constant of proportionality $W_{B}$. We define the total cost of bandwidth used between the VNF instance $f$ and the cloud as $S_{B}(f, c)$. As defined previously, $b$ is a constant representing the maximum core network delay when the bandwidth of $l_{c}$ is depleted. Then we have

$$
\begin{aligned}
S_{B}(f, c) & =w_{B}(c) B(f)=W_{B} t\left(l_{c}\right) B(f) \\
& =W_{B} \frac{T_{d}}{R\left(l_{c}\right)+b} B(f) \\
& =\frac{W_{B} T_{d} B(f)}{B\left(l_{c}\right)-\sum_{f^{\prime} \in F_{c}} B\left(f^{\prime}\right)+b} .
\end{aligned}
$$

From Equations (5.9), (5.10), and (5.11), the cost of offloading a VNF instance $f$ 
to the cloud, denoted by $S(f, c)$, is then calculated as

$$
\begin{aligned}
S(f, c) & =S_{U}(f, c)+S_{M}(f, c)+S_{B}(f, c) \\
& =\frac{W_{U} U(f)}{U(c)-\sum_{f^{\prime} \in F_{c}} U\left(f^{\prime}\right)+\delta}+\frac{W_{M} M(f)}{M(c)-\sum_{f^{\prime} \in F_{c}} M\left(f^{\prime}\right)+\delta}+\frac{W_{B} T_{d} B(f)}{B\left(l_{c}\right)-\sum_{f^{\prime} \in F_{c}} B\left(f^{\prime}\right)+b} .
\end{aligned}
$$

\subsubsection{Objective and 0-1 Integer Programming Formulation}

SP would like to reduce the total cost of deploying and running VNF instances for all users across the network edge. The VNF instances can be deployed either to the remote cloud location $c$, or to the pCPE location $v$. Based on where the VNF instances are offloaded, we identify two portions of costs offloading the VNF instances: 1) to the cloud, and 2) to $\mathrm{B} \& \mathrm{~B}$ nodes. The objective of the optimization is to minimize the total offloading cost of the SP.

\section{Variables:}

- $X(f, v)$ : A group of Boolean variables representing if each VNF instance $f$ is deployed on the B\&B node $v$.

- $X(f, c)$ : A group of Boolean variables representing if each VNF instance $f$ is deployed on the remote cloud $c$. 


$$
\begin{aligned}
& X(f, v)= \begin{cases}0, & f \text { not deployed on } v \\
1, & f \text { deployed on } v\end{cases} \\
& X(f, c)= \begin{cases}0, & f \text { not deployed on cloud; } \\
1, & f \text { deployed on cloud. }\end{cases}
\end{aligned}
$$

\section{Objective:}

$$
\text { Minimize } \sum_{f \in F} \sum_{v \in V} S(f, c) X(f, c)+S(f, v) X(f, v)
$$

\section{Constraints:}

$$
\begin{aligned}
& X(f, c)+\sum_{v \in V} X(f, v)=1, \forall f \in F, \\
& U(c)-\sum_{f \in F_{c}} U(f) \geq 0, \\
& M(c)-\sum_{f \in F_{c}} M(f) \geq 0, \\
& \frac{T_{d}}{R\left(l_{c}\right)+b} \leq T_{\max }(f), \forall f \in F_{c},
\end{aligned}
$$




$$
\begin{aligned}
& U(v)-\sum_{f \in F_{v}} U(f) \geq 0, \forall v \in V, \\
& M(v)-\sum_{f \in F_{v}} M(f) \geq 0, \forall v \in V .
\end{aligned}
$$

\section{Remarks:}

- Function (5.14) is the objective function. It minimizes the total cost of offloading VNFs instances to the cloud and to B\&B nodes.

- Constraint (5.15) ensures that every VNF instance $f \in F$ is only deployed at one place.

- Constraints (5.16) and (5.17) are the capacity bounds of the CPU and memory of the cloud. Each type of the three resources leveraged by all VNF instances offloaded to the cloud must not exceed the cloud's allocated resource capacity for the network edge.

- Constraint (5.18) sets the bottom line of the residue bandwidth for $l_{c}$ between the network edge and the cloud, which is essentially setting a limit for the number of VNFs offloaded to the cloud.

- Constraints (5.19) and (5.20) are the capacity bounds for CPU and memory of every pCPE node. Each type of the resources used by all VNFs offloaded to the vCPEs must not exceed these bounds. 


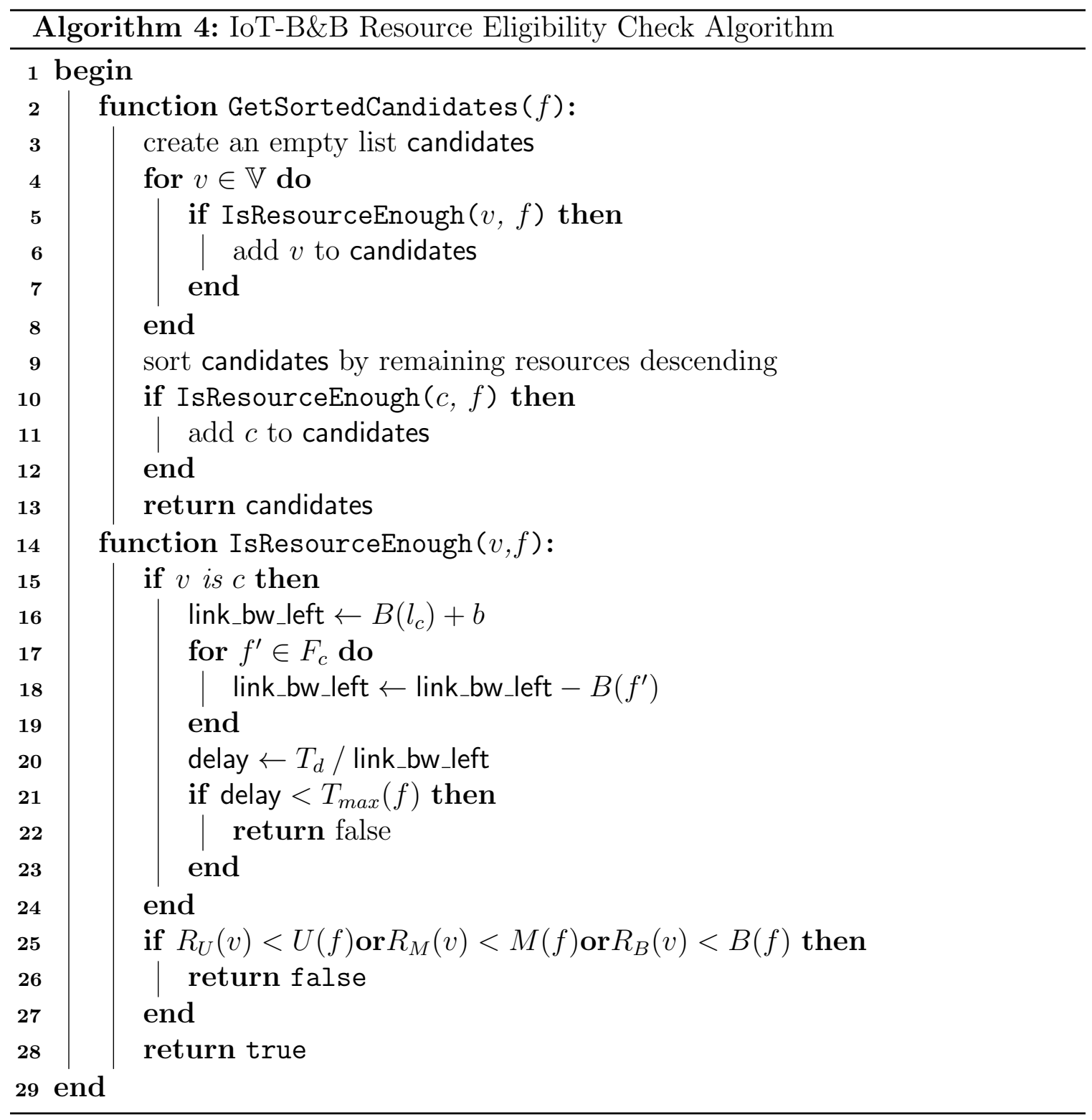




\subsection{IoT-B\&B Heuristic Placement Algorithm}

From the 0-1 integer programming in the previous section, we design a heuristic algorithm to achieve lower cost by choosing the first valid candidate place to deploy new VNF instances, after the candidate places are sorted by the remaining resources.

\subsubsection{Preliminary Resource Check}

For every request of deploying a new VNF instance, we first use Algorithm 3 to check the placement eligibility of every pCPE node, as well as the remote cloud. If the place does not meet the resource constraints of the instance, it will be excluded from the list of candidate places. By calling the function GetCandidates $(f)$, a list of candidate places will be returned from the input of a specific VNF instance $f$ and the current resource level. The list will be sorted by considering the lowest percentage of remaining resource type, in descending order. For example, if a pCPE node has $90 \%$ of vCPU left, but only $20 \%$ of memory left, then the remaining memory will be used for sorting.

\subsubsection{Cost Estimation}

With the list of eligible candidate places for a VNF instance $f$, we can further estimate the cost of $f$ deployed at each place. Algorithm 5 and 6 provide implementation of the cost model from Section 5.2. Algorithm 6 defines the function to choose the place for VNF instance $f$ at the lowest cost, namely ChoosePlace $(f)$. The function first calls GetCandidates $(f)$ in Algorithm 4 to get the places eligible for deploying $f$. Then for each eligible place, the cost is checked based on the type of the place based on Algorithm 5. If the place is the cloud, CLoudCost $(f)$ is invoked for cost; if the place is a $\mathrm{pCPE}$ node, the function $\operatorname{BnBCOST}(f, v)$ is called instead. After iterating 
all eligible places, the place with the lowest cost is selected and returned.

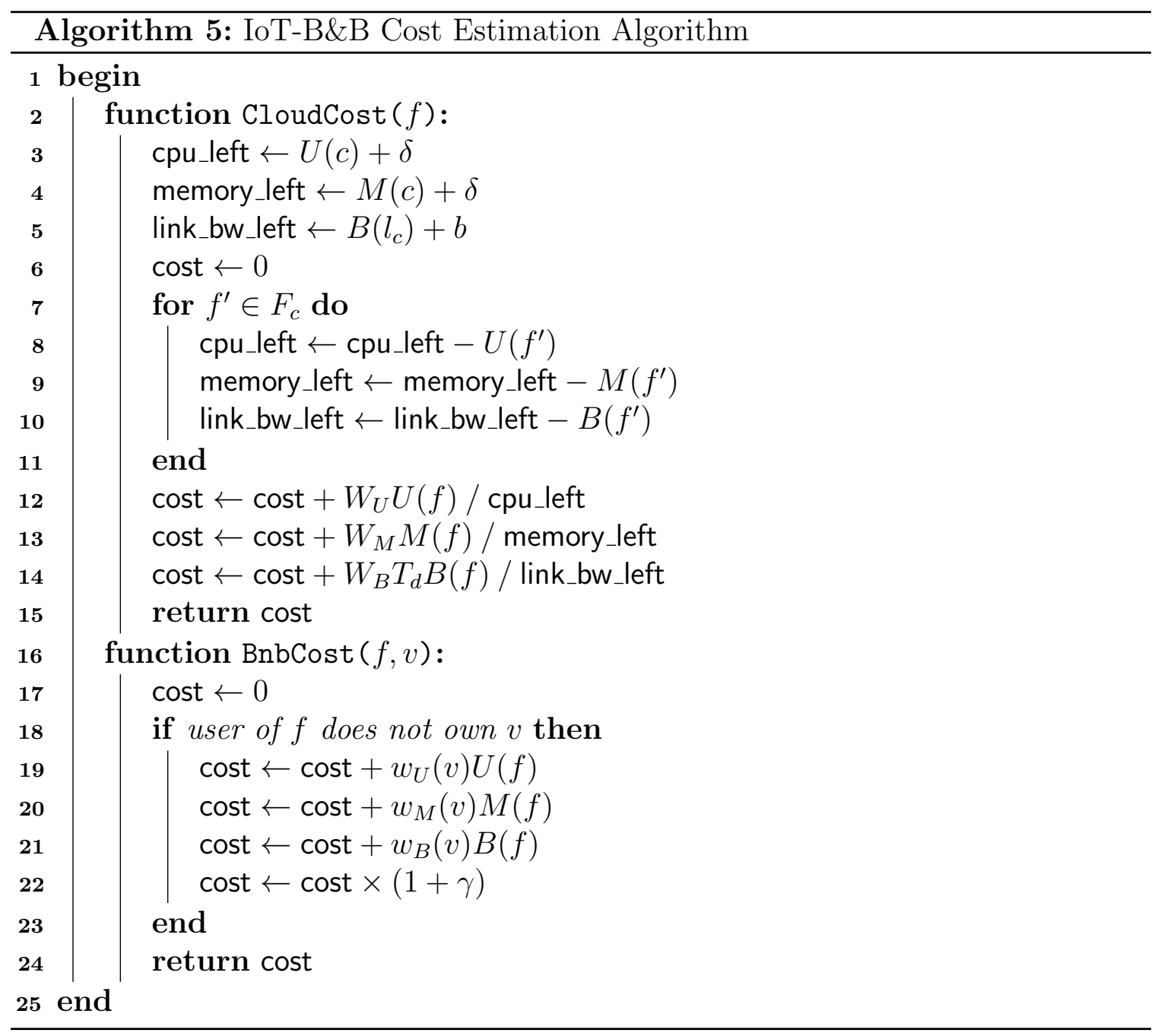

\subsubsection{Time Complexity}

Algorithm 4 has the time complexity of $O(n \log (n))$ because of sorting the pCPE nodes by remaining resources (assuming merge sort is used). Algorithm 5 has time complexity of $O(1)$.Algorithm 6 will always compare the first candidate pCPE node with the cloud and choose the destination with lower cost, which has the time complexity of $O(1)$. Combining the three algorithms, the time complexity of IoT-B\&B 


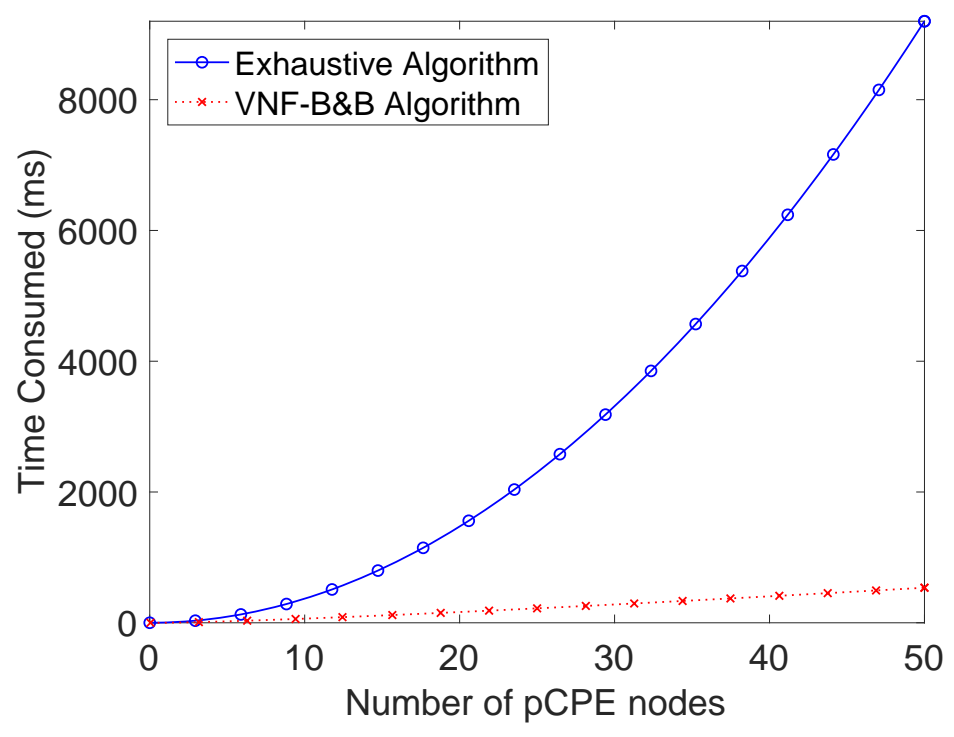

Figure 5.5: Time complexity comparison between IoT-B\&B algorithm and an exhaustive algorithm

Algorithm is $O(n \log (n))$.

If the exhaustive algorithm is used, which does not sort the candidate pCPE nodes, it would have to check all candidates and find out the one with the lowest cost. Such algorithm would increase the time complexity to $O\left(n^{2}\right)$. Figure 5.5 shows the time consumed using the two different algorithm above with up to $50 \mathrm{pCPE}$ nodes. From the results, we can see that IoT-B\&B algorithm scales well compared to the exhaustive algorithm, where the time consumed is less than $1000 \mathrm{~ms}$ for 50 nodes, while the exhaustive algorithm takes more than $9000 \mathrm{~ms}$.

\subsection{System Implementation}

We have implemented a system following the architecture illustrated in previous section. The system provides a platform to practice and evaluate the IoT-B\&B algorithm. 


\subsubsection{Hardware Configuration of pCPE Nodes}

For flexibility and scalability, we use VMs instead of bare metal machines as pCPE nodes. Up to $99 \mathrm{VMs}$ are deployed, each acts as a pCPE node with 8 Cores of CPU, 16 GB of memory, and 40 GB of disk space. Every pCPE node can communicate with any other one via a private virtual network, to mimic that these pCPE nodes are at the same network edge.

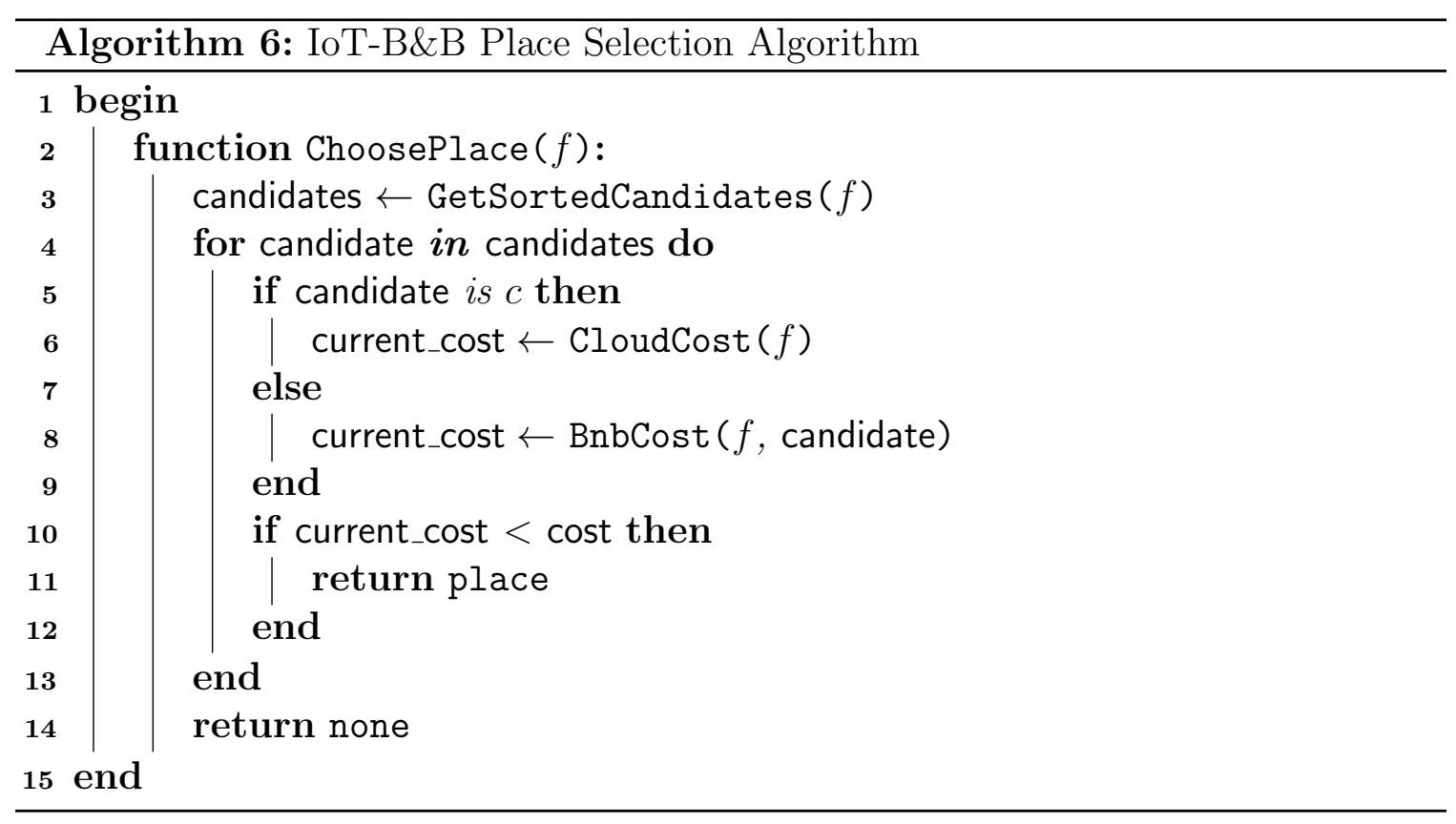

\subsubsection{NFVI Setup}

Each pCPE node has CentOS 7 115 installed as its operating system. It has its essential functionalities running as CPE. We use the OpenStack Kolla Project 116 to deploy OpenStack services across multiple pCPE nodes as well as PE, such that:

(a) The OpenStack services on a pCPE node runs as Docker containers. They can be spun up and torn down with minimal overhead. 

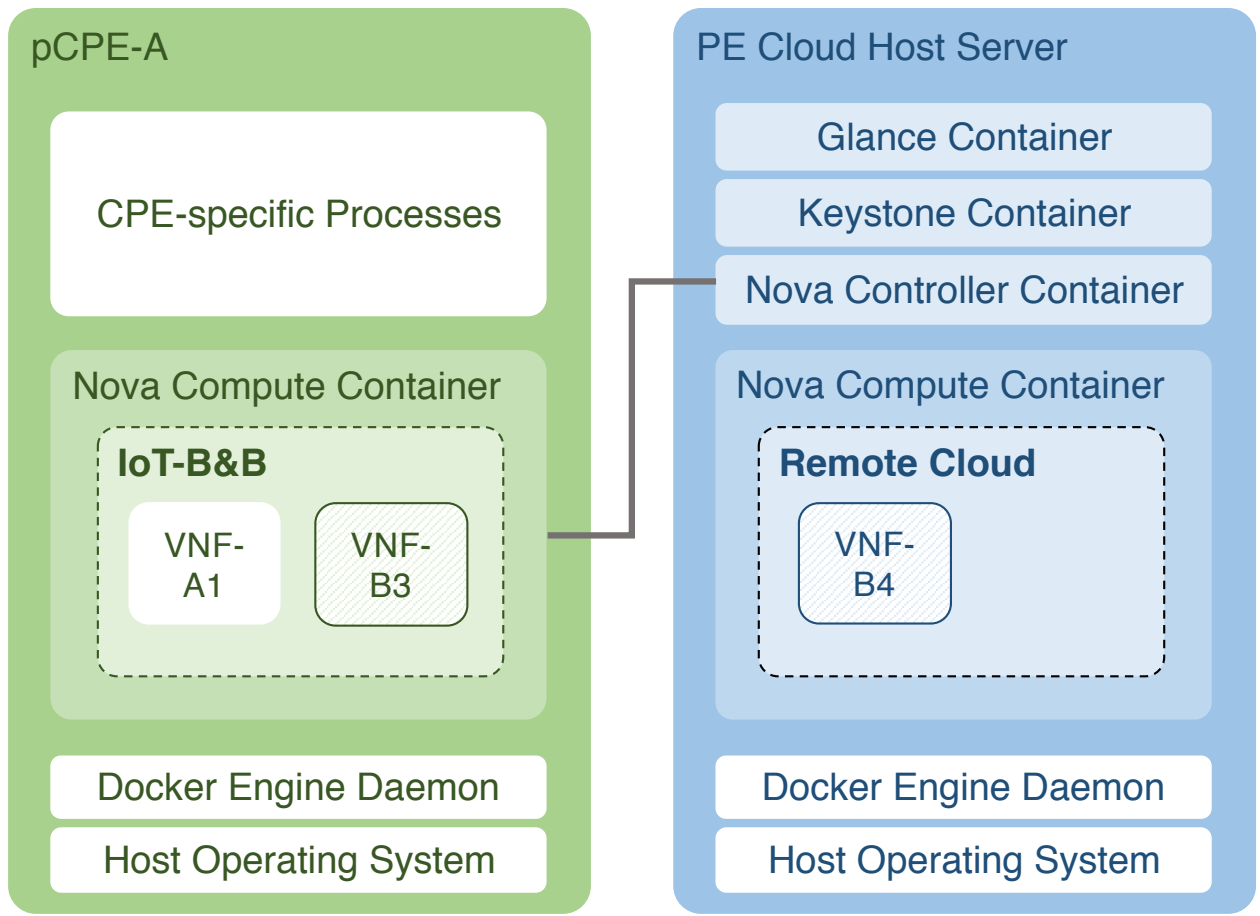

Figure 5.6: IoT-B\&B service backed by container-based OpenStack

(b) With container-based OpenStack services, a pCPE node can be converted to an OpenStack compute node and then register to the controller to be one of the available hosts.

(c) When the pCPE is no longer available to be a compute node due to high usage, the OpenStack services on it can be stopped to free up resources.

Figure 5.6 reflects the architecture we use to provision IoT-B\&B service upon container-based OpenStack.

\subsubsection{IoT-B\&B Algorithm As Filter Scheduler}

To leverage the proposed IoT-B\&B algorithm in the system, we implement it as a filter scheduler used by nova service, with the name VnfBnbFilter. The IoT-B\&B algorithm matches the filtering-weighting mechanism of the filter scheduler. 


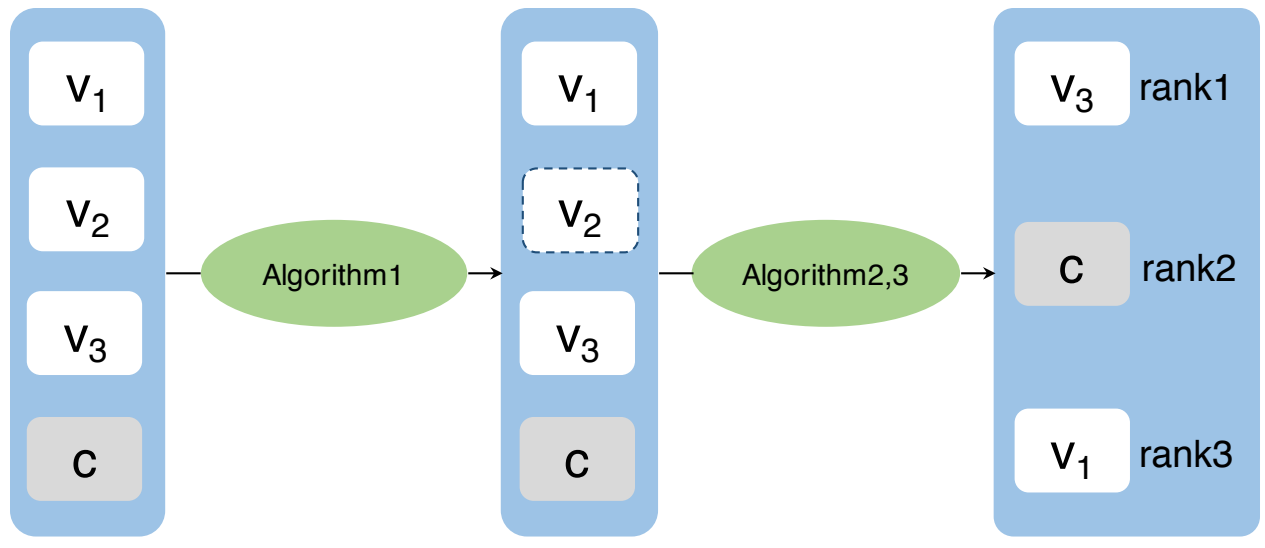

Figure 5.7: IoT-B\&B algorithm in OpenStack as a filter scheduler

Figure 5.7 shows how IoT-B\&B algorithm works as a filter scheduler to rank places to deploy. Suppose there are four places to choose: $v_{1}, v_{2}, v_{3}$ and $c$. Algorithm 4 is first invoked to filter out ineligible places that don't have enough resources. In the example, $v_{2}$ is filtered out as a result of resource shortage. Then Algorithms 5 and 6 are used to rank the places according to the cost to deploy the VNF instance. They determine that $v_{3}$ has the lowest cost to deploy the instance.

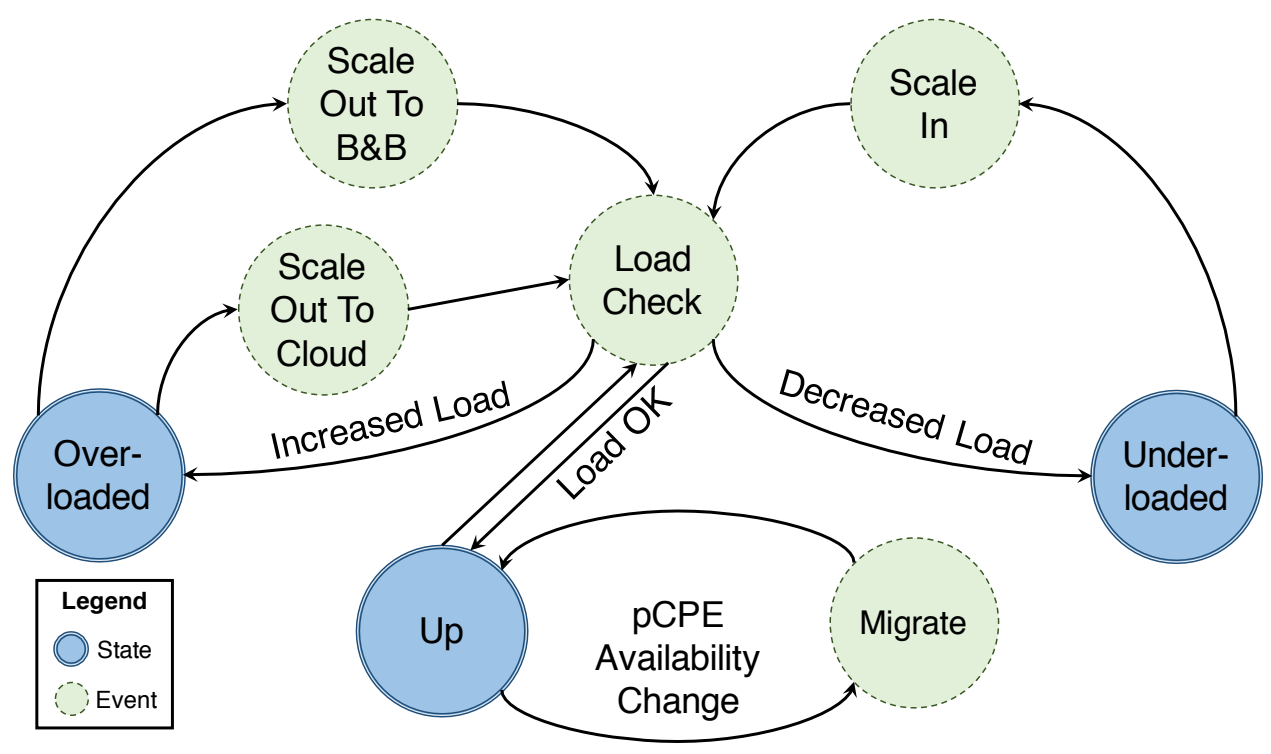

Figure 5.8: IoT-B\&B service system life cycle 


\subsubsection{System Life Cycle}

We define a set of system states and events for IoT-B\&B. The system transitions its state based on the events according to the actual demand, in order to adjust the scaling of the VNF. As Figure 5.8 shows, the states and events are listed as follows.

\section{Up State}

The state indicates the system is up and running as expected. System load level is acceptable to satisfy the needs. The system is expected stay in this state if it runs properly.

\section{Load Check Event}

This is the event to update the system load level. If the updated load level triggers a change, the system may enter Overloaded or Underloaded state, or remain in Up state, depending on the threshold to determine them.

\section{Overloaded State}

The state indicates the system is overloaded by higher volume of requests. A scale-out is pending. The placement scheduler will be invoked to determine the place to scale out: $\mathrm{B} \& \mathrm{~B}$ or the cloud, and then triggers the actual event to scale out.

\section{Scale Out To B\&B Event}

This is the event to trigger a new VM to be deployed on a B\&B, i.e., a CPE deployment. 


\section{Scale Out To Cloud Event}

This is the event to trigger a new VM to be scaled out in the remote cloud environment.

\section{Underloaded State}

The state indicates the system is underloaded because of lower volume of requests. A scale-in is pending. If the number of the VMs has already reached the minimum number required, then the system would not enter this state.

\section{Scale In Event}

This is the event to trigger an existing VM to be scaled in from either the $\mathrm{B} \& \mathrm{~B}$ or the remote cloud.

\section{Migrate Event}

This is the event that is triggered by pCPE availability changes. When a pCPE is no longer capable of hosting a VM because of higher usage from its user, it will cease to be a $\mathrm{B} \& \mathrm{~B}$ and be removed from the list of available hosts. Meanwhile, the migrate event will be added to the system to move the existing VMs deployed.

\subsubsection{Typical Use Cases}

With the definition of the system life cycle, we describe typical uses cases leveraging the IoT-B\&B algorithm. 


\section{Launch of New Application}

The dynamic nature of IoT-based services allows the user to launch new applications which are processed by new VNF instances. A new VNF instance does not automatically gets deployed on-site, i.e., the pCPE node of the its user. The reason can range from lack of enough resources, to higher cost being deployed on-site. The IoT-B\&B algorithm will be called to determine the place to deploy the new VNF instances.

\section{Scaling Out Due to Higher Load}

When the user applications have more significant activities, resulting in higher load of the existing instances, the system's periodical load check daemon will detect the load increase. If the load is above the threshold raising flags of performance, extra VNF instances are needed for processing the larger amount of requests. The IoT-B\&B algorithm will be called to determine the place to scale out new VNF instances.

\section{Migration For Lower Cost}

The VNF instances in the cloud may start with low cost. However, it does not last forever. As more VNF instances are deployed to the remote cloud, less resources are available and the unit resource becomes more expensive. At some point, a migration from the cloud to $\mathrm{B} \& \mathrm{~B}$ nodes, or vice versa, is reasonable to lower considerable cost.

\subsection{Numerical Results}

The numerical results based on simulations are shown in this section. We implement the algorithms using Java by writing a test suite according to the models and algorithms we have proposed. From the numerical results, our goals are to verify the benefits of leveraging $\mathrm{B} \& \mathrm{~B}$ nodes, compared to using a centralized cloud alone. 
Tables 5.1 lists the values of the constants used in the algorithms. Our simulations are done via the algorithm we have implemented using Java according to Algorithm 4.

Table 5.1: Constant configurations

\begin{tabular}{ll}
\hline Constant & Value \\
\hline$T_{d}$ & $50000 \mathrm{~ms}$ \\
$W_{U}$ & 1000 \\
$W_{M}$ & 1000 \\
$W_{B}$ & 1000 \\
$\delta$ & 1 \\
$b$ & 1 \\
$\gamma$ & 1 \\
\hline
\end{tabular}

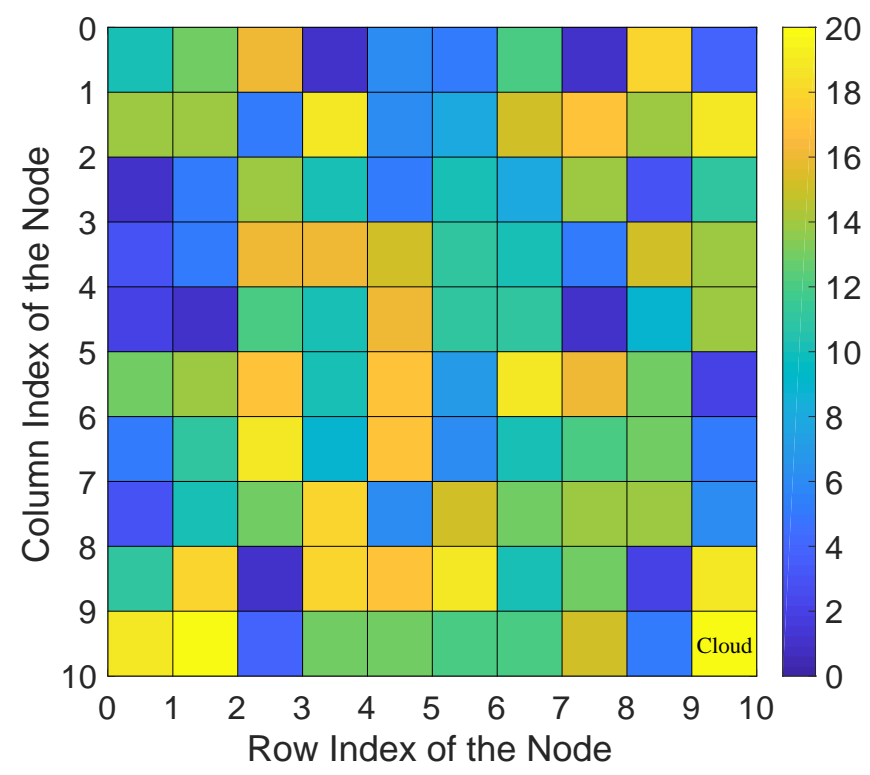

Figure 5.9: Initial resource levels of the 100 nodes used for experiments

\subsubsection{Host Nodes Setup}

We create $99 \mathrm{pCPE}$ nodes with random levels of initial resources. For each node, there are 8 vCPUs, 16384 MB RAM memory, and 10Gbps bandwidth. 
As all pCPE nodes and the cloud are used to deploy VNF instances, in our configuration, there is a total of 100 nodes for deployments. As seen in Figure 5.9, the nodes are arranged as a $10 \times 10$ matrix. Each node is represented by a cell, and is given a horizontal and a vertical coordinate between from 1 to 10. The last element, which has the coordinates $(10,10)$, represents the remote cloud. The colors of the cells reflect the remaining resource levels of the nodes. For the ease of demonstration, the CPU, memory, and bandwidth resources are all broken into 20 levels ranging from 1 to 20. Deploy VNF instances on a pCPE node follows the Law of the Minimum 117], meaning that the capacity of a pCPE node to host instances is determined by its scarcest resource. Therefore, we color the cells according the resource type of the lowest level of a node. For example, if the remaining CPU level of a node is 20, while the memory level is 3, the cell representing the node will be colored at Level 3. From the initial resource levels, it can be learned that the pCPE nodes have various levels of resources available. Meanwhile, the cloud starts with the maximum level of resources for deployment.

Table 5.2: Predefined flavor types for simulation

\begin{tabular}{lllll}
\hline Name & vCPU & Memory & Bandwidth Max De- \\
lay
\end{tabular}




\subsubsection{VNF Resource Requirement Profile (Flavor) Types}

We predefine 10 types of VNF resource requirement profiles (flavors), as shown in Table 5.2, with different requirements of resources and max delays allowed. A VNF instance to be deployed will have a flavor from the 10 predefined ones. Templating VNF flavors is based on the real use cases as users will need VNF instances from limited kinds of images for serving known functionalities.

\subsubsection{Placement Configuration Modes}

In order to compare the effectiveness of the IoT-B\&B algorithm, we configure the simulated system to keep deploying new VNF instances of a specific flavor with one of the three modes below, until the resources are depleted:

- Local Mode: Deploy only on the pCPE node the user owns. The cloud and B\&B nodes are not allowed.

- Local+Cloud Mode: Deploying locally on the pCPE node the user owns, as well as on the remote cloud.

- Local+Cloud+B\&B Mode: local, cloud, and B\&B deployments.

\subsubsection{Extended VNF Instance Capacity}

Figure 5.10 shows the numbers of deploying each of the 10 predefined flavors with the 3 modes and with the model proposed in 5.2. From the results of Figure 5.10 , we learn that the numbers of instances deployed for all 10 flavors have dramatically increased. Take F3 for example, in Local Mode, only 9 instances are deployed. When using Local+Cloud Mode, the number jumps to 85. For Local + Cloud $+B \mathscr{G} B$ Mode, the number skyrockets to 550 . When using using our model directly for placement, 


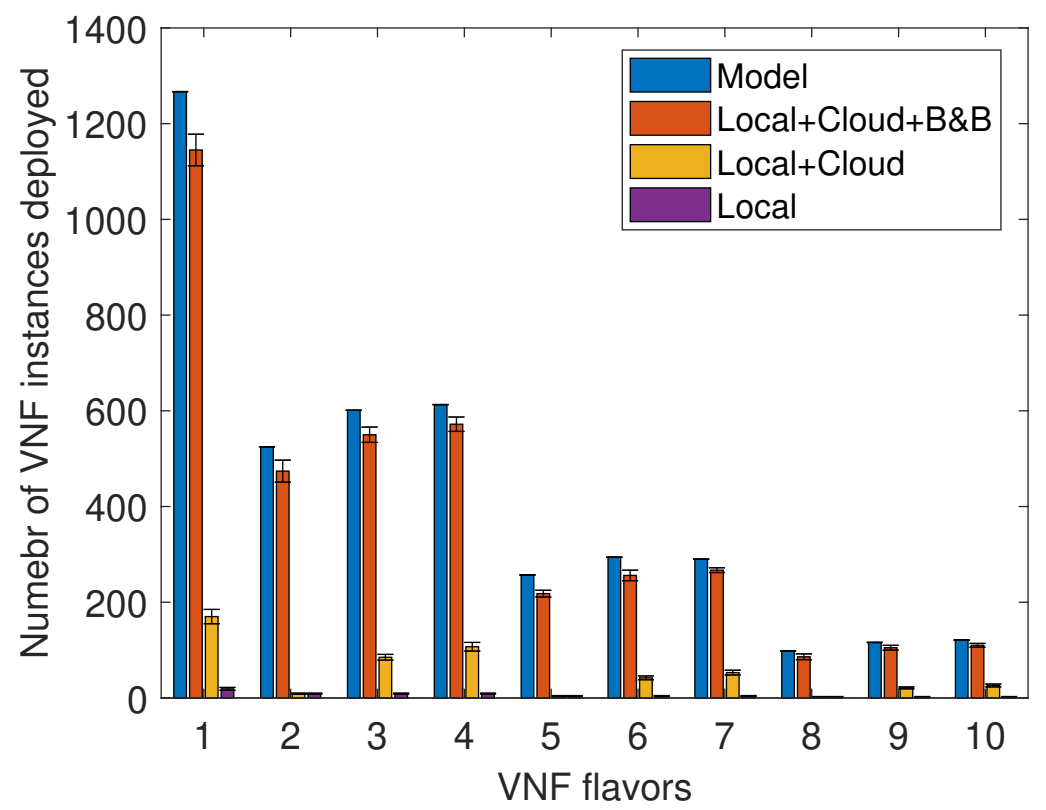

Figure 5.10: Total number of VNF instances deployed for the network edge with various flavors and placement modes and $95 \%$ confidence level
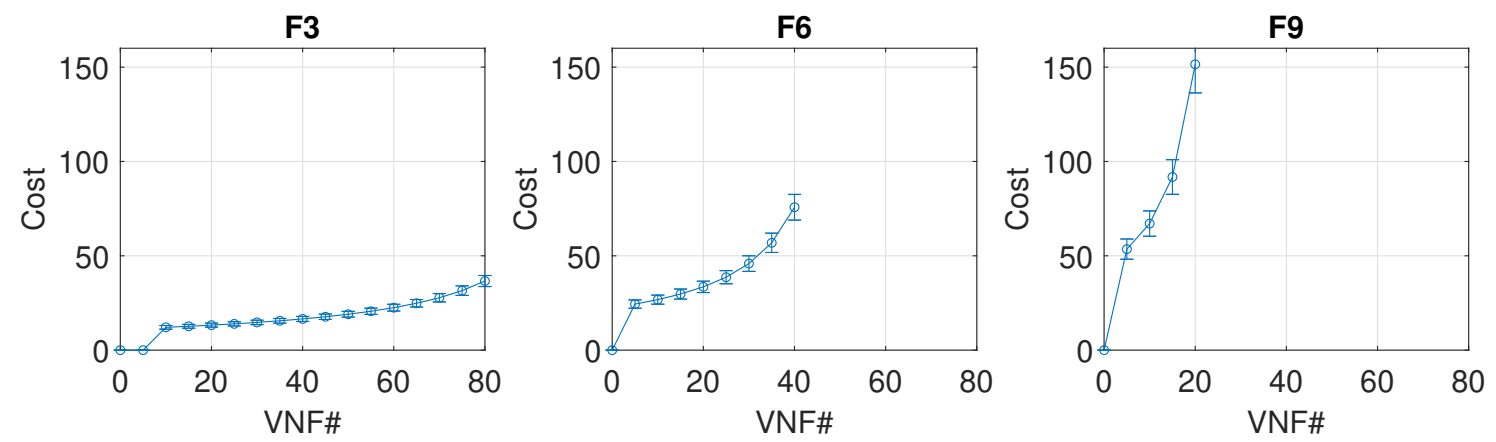

Figure 5.11: Cost hikes when the cloud load increases in Local+Cloud Mode with 95\% confidence level 
the result is 601 . We can also find that Local $+C l o u d+B \mathcal{B} B$ Mode has small losses of the total numbers of VMs deployed compared to the results directly returned by the model, which is acceptable. Therefore, the most beneficial part of the system is to extend the total capacity of hosting VNF instances. If using the remote cloud alone, the capacity of the cloud for VNF instances is limited by the core network delay, even if other resources are assumed to be unlimited. This bottleneck is greatly relieved by the $\mathrm{B} \& \mathrm{~B}$ nodes hosting instances, because the instances on $\mathrm{B} \& \mathrm{~B}$ nodes do not put extra traffic to the core network.
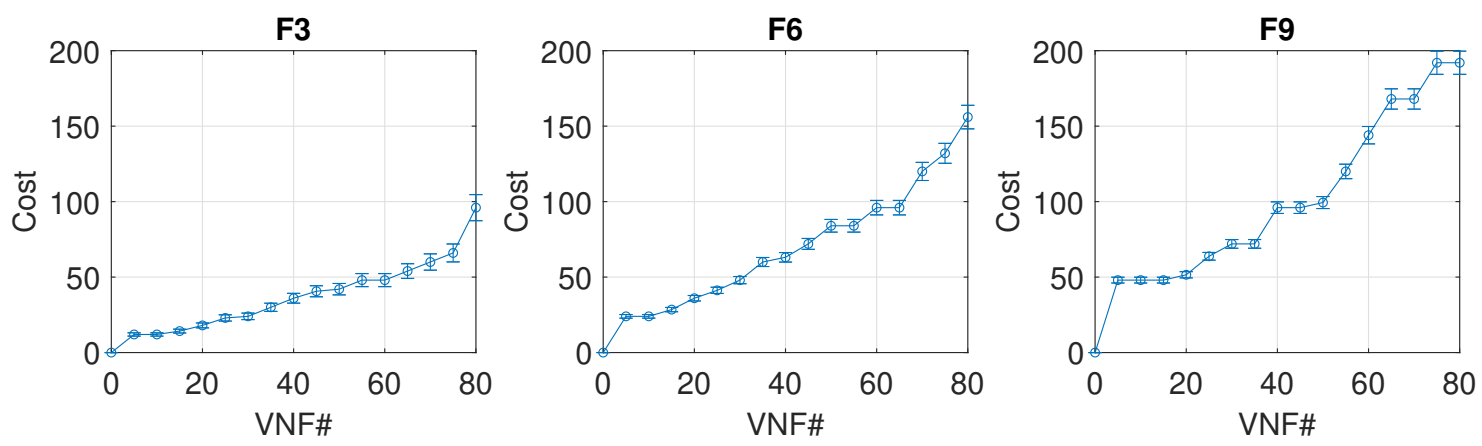

Figure 5.12: Cost hikes when the cloud load increases in Local+Cloud+BEBB Mode with $95 \%$ confidence level

\subsubsection{Cost Hike By Cloud Load Increase}

We pick the three flavors: F3, F6, and F9, to investigate the trends of cost increase as more VNF instances are deployed in the system. Figure 5.11 and 5.12 demonstrate the changes of costs to deploy a new VNF instance on the cloud in two different modes, as the numbers of deployed instances go up.

Using Local+Cloud Mode, the cost is first 0 as the instances are deployed on the local pCPE nodes. As the loads increase, the remote cloud starts to be picked and the cost to deploy an instance increases as the numbers of deployed instances climb. For F6 and F9, the numbers of deployed VNF instances stop at 42 and 21, respectively. 
Comparing Figure 5.12 with Figure 5.11, in Local+Cloud+B\&B Mode, the costs are lower when deploying the same numbers of instances in the system. For instance, when deploying 20 instances with the flavor F9, the cost using Local+Cloud Mode is about 140. Meanwhile, when deploying the same number of instances with the same flavor, the cost under Local + Cloud $+B \mathscr{E} B$ Mode is only around 50 .

The results above have demonstrated the ability of the B\&B nodes to redirect the load off the cloud and to reduce the overall cost, even if offering incentives to the users.
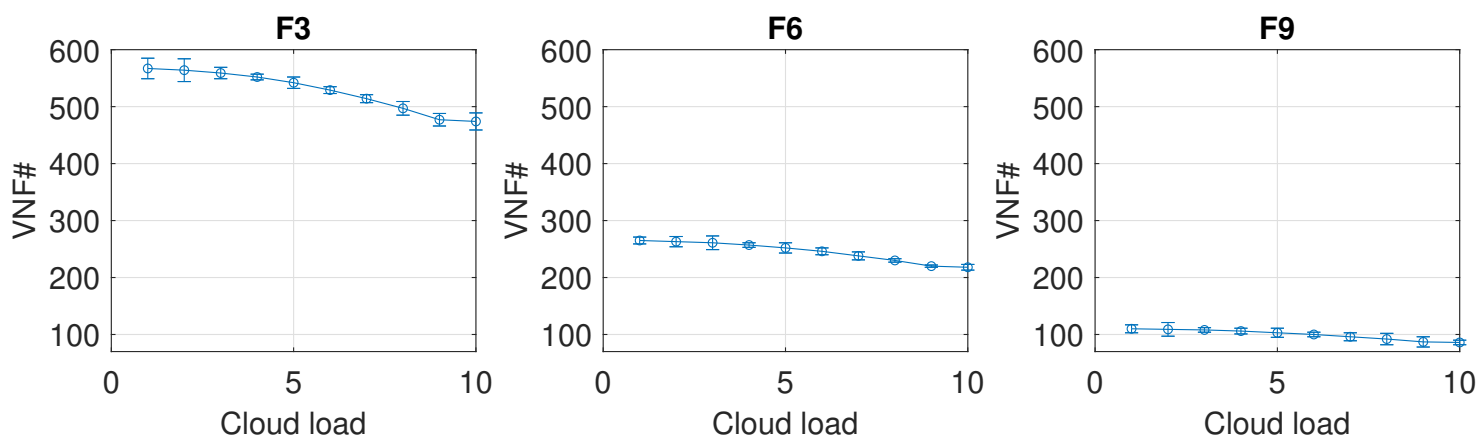

Figure 5.13: Cost changes when load increases due to tasks outside the target network edge with $95 \%$ confidence level

\subsubsection{Impact from Outside the Network Edge}

As discussed in Section 5.2, when the cloud load from outside the network edge gets higher, i.e., the value of $T_{d}$ is higher, the ability of the cloud hosting VNF instances may be reduced. To verify how much the impact will be, under $L o c a l+C l o u d+B \mathscr{E} B$ Mode and for the three flavors F3, F6, and F9, we increase the level of $T_{d}$ by 1 each time, and repeat the deployment for 10 times. The numbers of VNF instances deployed are shown in Figure 5.13. From the results, the capacity of the system is affected by the increase of $T_{d}$. However, the impact becomes less significant as the level of $T_{d}$ increases. With the considerable buffer of the B\&B nodes, the impact from 
$T_{d}$ is reduced.

\subsubsection{Remaining Resource Levels}

In Local + Cloud $+B \mathscr{E} B$ Mode, all B\&B nodes participate in hosting VNF instances. We examine there resource levels after the system resources are depleted. When all VNF instances deployed are of the flavor F1, the resource levels after the maximum number of instances are deployed are displayed in Figure 5.14 .

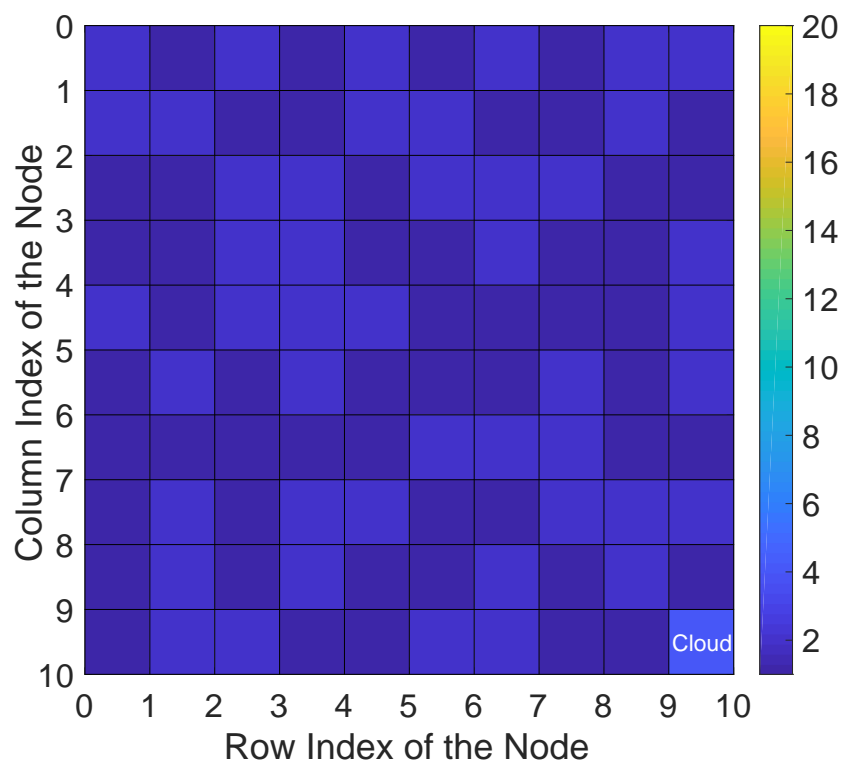

Figure 5.14: Remaining resource levels after all B\&B nodes used

The dark colors of all cells indicate that the remaining resource levels are low across all pCPE nodes. The cloud resource levels are also low because of the link/delay bottleneck from the network edge to the core network. The results have demonstrated the ability of the IoT-B\&B algorithm to extract the resources to deploy more instances following the best-effort basis. 


\subsection{Chapter Summary}

In this chapter, we have presented the architecture and the algorithms to share resources of pCPE nodes across the network edge. When a sharable pCPE node has enough resources, SP will utilize its free resources as a bed-and-breakfast place to deploy VNFs of other users from the same network edge for a certain period. The users can get incentives by allowing SP to leverage the free resources.

By applying the IoT-B\&B architecture, the capacity of VNF instances for the network edge is greatly increased. The cost of offloading to the centralized cloud is reduced. By keeping the VNFs at the network edge, the delay is reduced for better processing of real-time data burst from IoT devices. Meanwhile, the traffic load to the core network is substantially reduced with the same number of VNF instances deployed. 


\section{Chapter 6}

\section{Multi-vendor Edge Resource Sharing}

\subsection{Introduction}

The rapid advance of mobile edge computing (MEC) has been the last mile of enabling a shared, low-latency computational environment for multi-vendor mobile edge applications. MEC performs computing offloading, data storage, caching and processing, request distribution and service delivery from the mobile edge to end users [118. Applications with low latency tolerance, such as augmented reality (AR), video streaming, and online gaming, can deploy their services on the edge hosts at a cost, to achieve lower latency and better user experience [7].

As the market gets mature, there will be multiple 5G service providers (SPs) provisioning MEC services to cover the same area: bigger wholesale players will invest in infrastructure to actually build mobile edge base stations, while there will also be mobile virtual network operators (MVNOs) renting resources from the former. These SPs can collaborate with each other in several ways for better utilization of the resources at the edge: virtual SPs have to place mobile edge (ME) applications on one of the rented edge hosts, preferably with lower cost, regardless of SPs. On the other hand, MEC base stations from different SPs can share resources with each other 
to process bursting requests. Figure 6.1 gives an example of $3 \mathrm{ME}$ base stations from 3 different SPs in a certain service area with the potential of sharing resources among SPs.

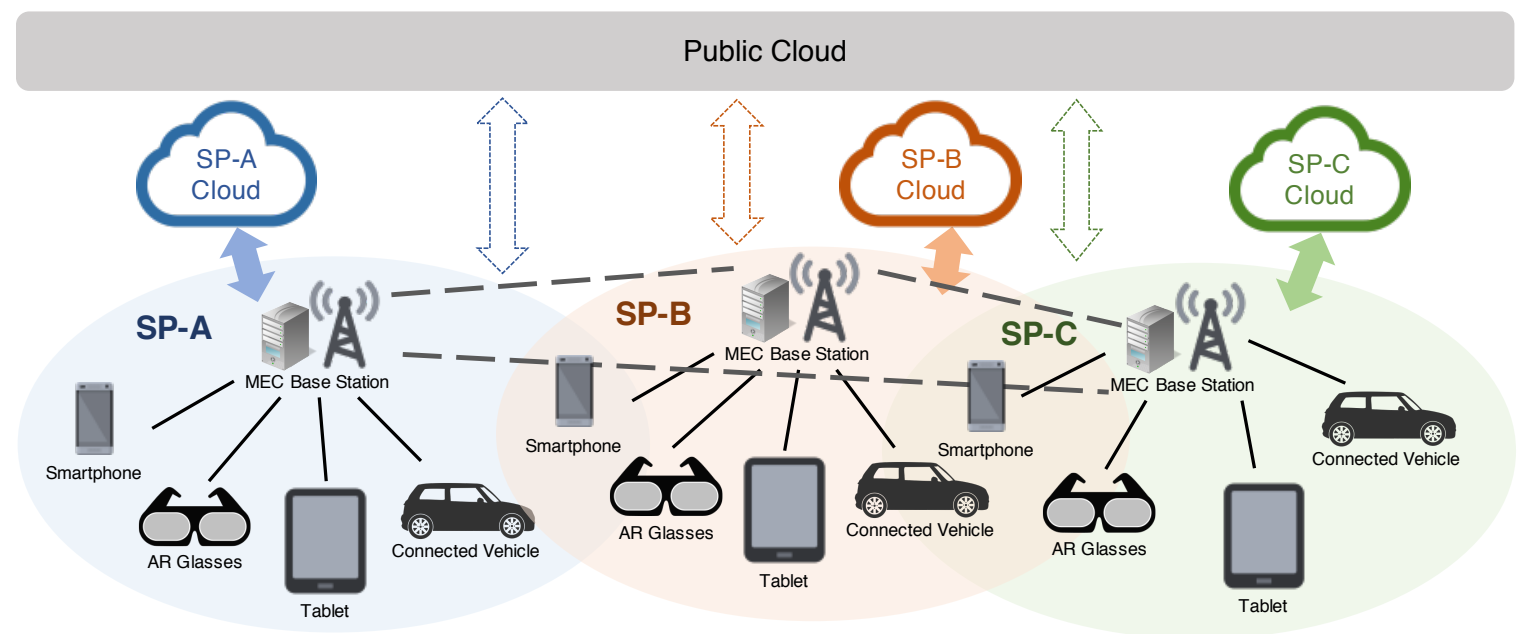

Figure 6.1: A MEC scenario in a certain service area with 3 ME base stations from 3 different SPs

For encouraging SPs to enroll their eligible MEC base stations and hosts in resource sharing, it is common to give incentives to SPs for contributing their resources of the hosts for hosting edge applications. Following the changing demand of end users, certain types of edge applications need to be deployed on, migrated to, or removed from an edge host, in order to meet the service requirement. By deploying the edge applications at the right places, the edge application provider will save costs, while providing high-quality service with low latency to the end users. Meanwhile, the edge host will collect incentives for its resources effectively used.

Clearly, the edge computing framework needs a placement service to dynamically check the user needs and the available edge hosts, and determine the placement or removal of edge applications. In datacenters, virtual machine (VM) placement has been well investigated, mainly with the focus of more efficient resource utilization and lower operational expense (OPEX). However, the collaboration of multiple SPs 
and mobile edge applications vendors are posing new challenges for ME application placement from the following aspects:

- A placement model has to make transparent and consistent selections of the best host for each request for edge computing resources. Moreover, the model has to take into consideration that a mobile edge application may require multiple services chained together at the edge.

- A trusted party is required to determine the best place for application deployment. When an edge application is deployed on a mobile edge host, the application vendor needs to pay for the usage of the host. The placement algorithm has to avoid affiliation to either SP to ensure a neutral decision is made strictly according to the resource and the cost. It may create conflicts of interest to put any SP involved into the position of making placement decisions: placing mobile edge applications onto the SP's own hosts would bring revenue for renting their resources.

- The application placement service needs to be steadily available. Both the mobile edge hosting service providers and the mobile edge application providers can constantly change. The placement service provider must remain in service regardless of the joining or quitting of vendors.

The challenges above urge a comprehensive solution uniting all SPs and their edge hosts without bias. In this chapter, we present an architecture combined with its algorithm, namely EdgeChain, to create a decentralized placement service for mobile edge application that does not require trust to any party, i.e., trustless placement service. Compared with current placement solutions, EdgeChain has the following contributions: 
- A cost model is presented as a integer programming problem, factoring in the pricing of edge hosts, latency, and service chaining.

- We develop a heuristic placement algorithm based on the proposed cost model with the consideration of efficiency for running by the blockchain.

We divide the contents into the following sections. Section 6.2 formulates the problem. Section 6.3 proposes the heuristic EdgeChain placement algorithm based on the problem formulation. Then the simulation results are shown in Section 6.4. Section 6.6 summarizes this chapter.

Table 6.1: Parties involved in a MEC placement scenario

\begin{tabular}{ll}
\hline Party & Description \\
\hline Users & $\begin{array}{l}\text { Subscribers of applications and services over 5G networks with MEC } \\
\text { enabled. } \\
\text { MECSPs service providers, who deliver MEC hosting services that } \\
\text { can run MEApps at the network edge, close to end users. Exam- } \\
\text { ples include telecommunication companies like Rogers and Telus in } \\
\text { Canada. }\end{array}$ \\
MEAVs & $\begin{array}{l}\text { Mobile edge application vendors, who provide MEApps and services } \\
\text { to end users. For instance, a company selling AR services. }\end{array}$ \\
MEApps & $\begin{array}{l}\text { MEApps stand for mobile edge applications provided by MEAVs. } \\
\text { Servers that belong to different MECSPs to provide hosting service } \\
\text { of MEApps. }\end{array}$ \\
HostLinks & $\begin{array}{l}\text { Network links between hosts, regardless of which MECSP they be- } \\
\text { long to. }\end{array}$ \\
WppLinks & $\begin{array}{l}\text { When MEApps are chained together, virtual links will be established } \\
\text { for data transmissions traveling through the chain. }\end{array}$ \\
\hline
\end{tabular}

\subsection{Problem Formulation}

We first list all parties involved in a MEC placement scenario in Table 6.1. The problem is formulated from a $M E A V$ 's point of view: MEApps are direct consumers of the computing resources in the MEC environment, because a $M E A V$ needs to 
pay MECSPs for hosting its applications in order to serve their users and meet the latency requirement. Each $M E A p p$ is equivalent to a virtual machine (VM) deployed on a MEHost. MEApps provided by different $M E A V$ can be combined as a service chain to provide comprehensive services. A service chain may span multiple MEAVs. In this case, revenues generated by the service chain can be distributed according to the usage of each MEApp on the service chain. For instance, a full-fledged AR service can load real-time navigation information from an online map application, while it can also load promotions of a shopping mall nearby from the mall's application. The navigation data is collected by the online map application, and the shopping mall application gets paid if the user "clicks" the links of the promotions.

The notations used in formulating the problem is shown in List of Symbols. Define a chained service $s$ as a forwarding graph [69] $G_{s}=\left(\mathbb{V}_{s}, \mathbb{L}_{s}\right)$, where $\mathbb{V}_{s}$ is the set of all MEApps contributing to the service, and $\mathbb{L}_{s}$ is the set of all AppLinks connecting applications together. A MEApp is denoted by $v \in \mathbb{V}_{s}$, and an AppLink between two MEApps is denoted by $l \in \mathbb{L}_{s}$.

The chained service is deployed on a graph of connected MEHosts $G_{h}=(\mathbb{H}, \mathbb{E})$, where $\mathbb{H}$ is the set of all MEHosts owned by various MECSPs and $\mathbb{E}$ is the set of all HostLinks. A MEHost is denoted by $h \in \mathbb{H}$, and a HostLink between two MEHosts is denoted by $e \in \mathbb{E}$. The HostLinks can be either physical or virtual links with fixed capacities and latencies. In Chapter 4. Figure 4.5 demonstrates an example of service chain with $3 \mathrm{ME}$ service chains sharing the services provided by MEApps.

Suppose in a certain service area, there are $n_{s}$ users from various MECSPs requesting the same chained service $s$ from a MEAV. We use $m$ to denote a MECSP and $h_{m}$ for a MEHost that belongs to $m$. Define an assigning function $x_{v h_{m}}$, whose 
value is 1 if $\mathrm{VM} v$ is assigned to Host $h_{m}, 0$ otherwise.

$$
x_{v h_{m}} \triangleq \begin{cases}1, & v \text { is deployed on } h_{m} \\ 0, & \text { otherwise }\end{cases}
$$

Define a binary indicator of an AppLink between two chained MEApps in s, denoted by $L\left(v_{h_{i}}, v_{h_{j}}\right)$, such that

$$
L\left(v, v^{\prime}\right) \triangleq \begin{cases}1, & l \in \mathbb{L}_{s} \text { exists between } v \text { and } v^{\prime} \\ 0, & \text { otherwise. }\end{cases}
$$

Also, we use $e_{i j}$ to represent the HostLink between $h_{i}$ and $h_{j}$. The cost of deploying $s$ is the sum of the cost of deploying each MEApp $v$ of the service and the cost of the traffic between each two adjacent MEApps in the service chain. It can be shown by

$$
\begin{aligned}
c_{s}= & \sum_{h_{m} \in H} \sum_{v \in \mathbb{V}_{s}} c_{v h_{m}} x_{v h_{m}} \\
& +\sum_{h_{i}, h_{j} \in H} \sum_{v, v^{\prime} \in \mathbb{V}_{s}} c_{v h_{i}, v^{\prime} h_{j}} x_{v h_{i}} x_{v^{\prime} h_{j}} L\left(v, v^{\prime}\right),
\end{aligned}
$$

where $c_{s}$ represents the cost of deploying $s$ and $c_{v h_{m}}$ is for the cost of a MEApp $v$ deployed on a MEHost $h_{m}$. We assume that the pricing scheme for the same MECSP is the same across all of its hosts. For a MEHost $h_{m}$, define its basic unit resource price, which is the unit price of serving its own subscribers, as $\gamma_{m}$. When $h_{m}$ is serving users of other MECSPs, it charges a premium of $\delta_{m}$ for its unit resource, as the return for doing courtesy for its partners. Therefore, the shared unit resource price of $h_{m}$ can be represented by $\left(\gamma_{m}+\delta_{m}\right)$. Define $C_{h_{m}}$ and $M_{h_{m}}$ to be the capacity of vCPU and memory provided by $h_{m}$. Define $C_{v}$ and $M_{v}$ as the vCPU and memory consumed by $v$. Define $P_{m}$ to be the percentage of the users using the service chain 
$s$ via networks of the MECSP $m$. Depending on the numbers of active users for each $M E C S P$, the total cost for the $M E A V$ to place its MEApp $v$ onto a host of $m$ is the cost incurred by users of $m$ plus the cost by users of other MECSPs:

$$
\begin{aligned}
c_{v h_{m}}= & n_{s}\left(C_{v}+M_{v}\right) P_{m} \gamma_{m} \\
& +n_{s}\left(C_{v}+M_{v}\right)\left(1-P_{m}\right)\left(\gamma_{m}+\delta_{m}\right) \\
= & n_{s}\left(C_{v}+M_{v}\right)\left[P_{m} \gamma_{m}+\left(1-P_{m}\right)\left(\gamma_{m}+\delta_{m}\right)\right] \\
= & n_{s}\left(C_{v}+M_{v}\right)\left[\gamma_{m}+\left(1-P_{m}\right) \delta_{m}\right] .
\end{aligned}
$$

When a request from a user for a service chain arrives, the blockchain would know the MECSP from which the user subscribes. For the same placement decision, the value $c_{s}$ can significantly differ over changing distribution of users. An example can be two MECSPs $m_{1}$ and $m_{2}$, each with one host $h_{m_{1}}$ and $h_{m_{2}}$. If all users are subscribers of $m_{1}$ and all MEApps are placed on MEHosts of $m_{1}$, then the cost payable by the $M E A V \mathrm{~s}$ would be lower than if all users were subscribers of $m_{2}$.

\subsubsection{HostLink Unit Price}

When it comes to the cost modeling of a link between two MEHosts, link availability is an important part for the service consistency of the MEApps. If a heavily used HostLink is down, consequences can be catastrophic: even if all individual MEApps are running, the traffic would not be able to flow through between one or more pairs of MEApps and the service chain would not be functional. For each HostLink $e_{i j}$, there can be one or more AppLinks sharing its bandwidth. HostLink outages require migrating the MEApps if they cannot be fixed in time. Therefore, HostLink availability has significant influence on possible $M E A p p$ migrations and the potential costs incurred.

The link unit price of a HostLink $e_{i j}$, denoted by $\zeta_{e_{i j}}$, is then defined to describe 
how much to use the HostLink $e_{i j}$. The following two parameters will determine $\zeta_{e_{i j}}$.

The first parameter is $L\left(v_{h_{i}}, v_{h_{j}}\right)$ as defined in Eqn. 6.2). The more AppLinks a HostLink carries, the more vital and expensive it becomes. The reason behind this ranking parameter is the potential consequence of migration: failure of a HostLink used by many VMs would lead to massive migration of all MEApps connected by that HostLink, which would be more disruptive to the service chain.

The other parameter $B_{V}\left(e_{i j}\right)$ is the total bandwidth consumed by traffic between MEApps on the two hosts. It is selected because larger bandwidth usages would cause challenges at the time of migration: it can be hard to find another link with enough capacity.

$$
B_{V}\left(e_{i j}\right) \triangleq\left[\sum_{v_{h_{i}}, v_{h_{j}}, h_{i} \neq h_{j}} B\left(v_{h_{i}}, v_{h_{j}}\right)\right] .
$$

Combining the two parameters, we define the unit price $\zeta_{e_{i j}}$ of a HostLink $e_{i j}$, as the factor of the number of AppLinks between two hosts times the factor of traffic flowing through these links:

$$
\zeta_{e_{i j}}=\frac{\left[\sum_{v_{h_{i}}, v_{h_{j}}, h_{i} \neq h_{j}} L\left(v_{h_{i}}, v_{h_{j}}\right)\right]}{N_{e_{i j}}} \frac{B_{V}\left(e_{i j}\right)}{B\left(e_{i j}\right)},
$$

where $N_{e_{i j}}$ is the maximum number of virtual links possible on $e_{i j}$. Therefore, $\zeta_{e_{i j}} \in$ $[0,1]$. The value of $\zeta_{e_{i j}}$ will rise to mark up a link's importance given it is either occupied by more pairs of VMs, or there is more traffic assigned to $e_{i j}$, or both. The cost of any two MEApps is then the sum of the cost serving users that belong to the MECSPs owning $h_{i}$ and $h_{j}$ and the cost serving other users timed by the price factor 
$\kappa_{e_{i j}}:$

$$
\begin{aligned}
c_{v h_{i}, v^{\prime} h_{j}}= & n_{s} \zeta_{e_{i j}}\left(P_{m_{h_{i}}}+P_{m_{h_{j}}}\right) \kappa_{m_{h_{i}} m_{h_{j}}} \\
& +n_{s} \zeta_{e_{i j}}\left(1-P_{m_{h_{i}}}-P_{m_{h_{j}}}\right)\left(\kappa_{m_{h_{i}} m_{h_{j}}}+\sigma_{m_{h_{i}} m_{h_{j}}}\right) \\
= & n_{s} \zeta_{e_{i j}}\left[\left(P_{m_{h_{i}}}+P_{m_{h_{j}}}\right) \kappa_{m_{h_{i}} m_{h_{j}}}\right. \\
& \left.+\left(1-P_{m_{h_{i}}}-P_{m_{h_{j}}}\right)\left(\kappa_{m_{h_{i}} m_{h_{j}}}+\sigma_{m_{h_{i}} m_{h_{j}}}\right)\right] .
\end{aligned}
$$

\subsubsection{HostLink latency}

Define the latency of the link $e_{i j}$ to be $t_{e_{i j}}$. For a service chain $s$, the total latency $t_{s}$ is then

$$
t_{s}=\sum_{h_{i}, h_{j} \in \mathbb{H}} \sum_{v_{h_{i}}, v_{h_{j}} \in \mathbb{V}_{s}} L\left(v_{h_{i}}, v_{h_{j}}\right) x_{v h_{i}} x_{v h_{j}} t_{e_{i j}} .
$$

In the equation above, $t_{e_{i j}}$ is a constant depending on the particular $e_{i j}$. If $h_{i}=h_{j}$, then we consider the latency to be 0 , since no actual HostLink is used for data transmission between the two MEApps. Define the maximum latency allowed for the service chain $s$ is $T_{s}$. Then there must be $t_{s} \leq T_{s}$ to meet the latency requirement.

\subsubsection{Integer Programming Formulation}

The problem is formulated as a integer programming as $x_{v h_{m}}$ and $L\left(v, v^{\prime}\right)$ used in the objective function can only be integers. Define $\mathbb{V}_{h}$ as the set of all MEApps deployed on the MEHost $h$. The objective is to minimize the total cost of the service chain $s$ to provide service with the lowest cost to the end user. As discussed in Section 6.2, the optimization is to minimize the costs on MEHosts and HostLinks for all MEApps of $s$. 
Minimize

$$
\begin{aligned}
c_{s}= & \sum_{h_{m} \in \mathbb{H}} \sum_{v \in \mathbb{V}_{s}} c_{v h_{m}} x_{v h_{m}} \\
& +\sum_{h_{i}, h_{j} \in \mathbb{H}} \sum_{v, v^{\prime} \in \mathbb{V}_{s}} c_{v h_{i}, v^{\prime} h_{j}} x_{v h_{i}} x_{v^{\prime} h_{j}} L\left(v, v^{\prime}\right) \\
= & \sum_{h_{m} \in \mathbb{H}} \sum_{v \in \mathbb{V}_{s}} x_{v h_{m}} n_{s}\left(C_{v}+M_{v}\right)\left[\gamma_{m}+\left(1-P_{m}\right) \delta_{m}\right] \\
& +\sum_{h_{i}, h_{j} \in \mathbb{H}} \sum_{v, v^{\prime} \in \mathbb{V}_{s}} n_{s} \zeta_{e_{i j}}\left[\left(P_{m_{h_{i}}}+P_{m_{h_{j}}}\right) \kappa_{m_{h_{i}} m_{h_{j}}}\right. \\
& \left.+\left(1-P_{m_{h_{i}}}-P_{m_{h_{j}}}\right)\left(\kappa_{m_{h_{i}} m_{h_{j}}}+\sigma_{m_{h_{i}} m_{h_{j}}}\right)\right] L\left(v, v^{\prime}\right),
\end{aligned}
$$

$$
\begin{aligned}
& \text { w.r.t. } \quad x_{v h_{m}}, \\
& \text { s.t. } \quad B\left(e_{i j}\right) \geq \sum_{v_{h_{i}}, v_{h_{j}}, h_{i} \neq h_{j}} B\left(v_{h_{i}}, v_{h_{j}}\right), \\
& C_{h} \geq \sum_{v \in \mathbb{V}_{h}} C_{v},
\end{aligned}
$$$$
M_{h} \geq \sum_{v \in \mathbb{V}_{h}} M_{v},
$$

$$
\sum_{h_{i}, h_{j} \in \mathbb{H}} \sum_{v_{h_{i}}, v_{h_{j}} \in \mathbb{V}_{s}} L\left(v_{h_{i}}, v_{h_{j}}\right) x_{v h_{i}} x_{v h_{j}} t_{e_{i j}} \leq T_{s} .
$$




\section{Remarks}

- Function 6.9) is the objective function. It minimizes the cost of all MEApps and AppLinks by using less hosts, while not exhausting them. The decision variable is $x_{v h_{m}}$ which indicates if $v$ is deployed on $h_{m}$.

- Constraint (6.10) is the HostLink bandwidth capacity bounds between each two hosts. Traffic transmitted between any two hosts $h_{i}$ and $h_{j}$ must not exceed the corresponding bandwidth capacity $B\left(e_{i j}\right)$.

- Constraints 6.11) and 6.12 are the CPU and memory capacity bounds for each MEHost. The CPU and memory used by MEApps coordinating with each other and by intra-host communications must not exceed $C_{h}$ and $M_{h}$.

- Constraint (6.13) is the latency requirement of the service chain $s$ to ensure that the total latency of $s$ must not exceed the maximum latency allowed $T_{s}$.

\subsection{The EdgeChain Placement Algorithm}

The formulation presented in the previous section is NP-hard [109. It may not be computationally feasible when attempting to solve it in large scale. To apply our model to real-world scenarios, we design a heuristic algorithm called EdgeChain to achieve suboptimal results by applying a hybrid strategy of best-fit and first-fit decreasing algorithm. The pseudo code of the algorithm is shown in Algorithm 7 .

\subsubsection{Processing Order and selection of MEHosts}

The EdgeChain algorithm runs on each mining node based on the Ethereum platform. The algorithm retrieves its input information from the blockchain, as all transactions 


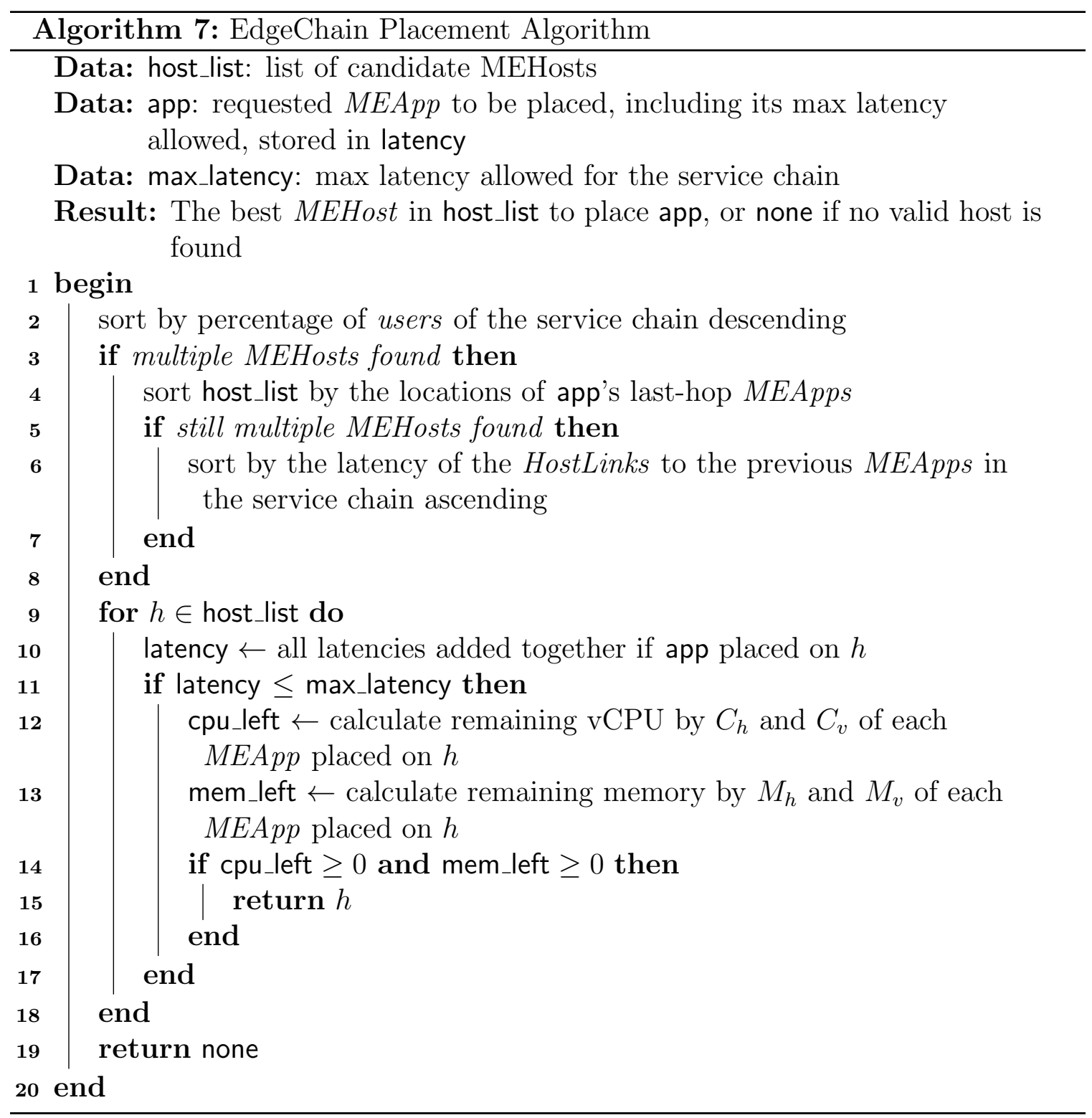


and updates are recorded on the blockchain. The EdgeChain algorithm will select the MEHosts following the steps below.

\section{Users}

Sort all MEHosts by the percentage of users of the service chain. For each MEApp on the service chain, consider which MECSP has most users using it. Then MEHosts with the same MECSP will have higher ranks to deploy this MEApp. Since all MEHosts of the same MECSP have the same unit resource cost, the MEApp can be placed on any of the MEHosts that belongs to the best MECSP, to avoid the situation that too many MEApps are concentrated on one MEHost.

\section{Last-hop MEApp}

For MEHosts given higher priority in the previous step, sort by the locations of lasthop MEApps. MEHosts hosting the previous-hop MEApps will be considered first. This step is to reduce the traffic cost between different MECSPs.

\section{Latency}

For MEHosts given higher priority in the previous step, sort by the latency of the HostLinks to the previous MEApps in the service chain. MEHosts with lower latency will be considered first.

After the list of candidate MEHosts are sorted according to the steps above, the algorithm iterates the list and pick the first valid MEHost that has enough resources to place the MEApp, as well as meeting the latency requirement of the service chain. 


\subsection{Numerical Results}

In this section, we illustrate the numerical results of the MEC placement cost changes based on varying mobile edge application user cases using CloudSim [119]. Our sim-

ulations are done via the placement algorithm we have implemented using Java according to Algorithm 7. To clearly demonstrate the focused trends, the following assumptions are made to simplify the modeling of the problem without losing generality. We first discuss the placement results output by the EdgeChain algorithm for the same service chain on the same set of MEHosts.

1. The unit costs of the CPU and memory of all hosts for the same MECSP are the same.

2. Costs of network bandwidth for all links follow the same unit price.

3. One mobile edge application includes the same type of VMs with the same CPU, memory and network bandwidth requirements.

4. A request from the user will be processed by one VM, while the VM may communicate with other VMs to exchange information.

\subsubsection{Parameters}

With the assumptions above, we choose parameters for our placement model to evaluate the performance and the facts under different circumstances. First, we choose a MEC service scenario of 3 MECSPs $m_{1}, m_{2}$, and $m_{3}$, each with 3 MEHosts, where $h_{1}, h_{2}, h_{3}$ belong to $m_{1}, h_{4}, h_{5}, h_{6}$ belong to $m_{2}$, and $h_{7}, h_{8}, h_{9}$ belong to $m_{3}$.

Three identical requested service chain, each with 5 MEApps is to be placed. The MEApps of each service chain are denoted by $v_{1}, v_{2}, v_{3}, v_{4}$, and $v_{5}$. The service chain starts from $v_{1}$ and ends at $v_{5}: v_{1} \rightarrow v_{2} \rightarrow v_{3} \rightarrow v_{4} \rightarrow v_{5}$. We assume that all MEApps 
have the same CPU, memory and bandwidth requirements, which are shown in Table 6.2 , along with other parameters.

Table 6.2: Parameters for the MEC scenario

\begin{tabular}{ll|ll}
\hline Parameter & Value & Parameter & Value \\
\hline$C_{v}$ & 2 vCPUs & $M_{v}$ & $2048 \mathrm{MB}$ \\
$C_{h}$ & 64 vCPUs & $M_{h}$ & $65536 \mathrm{MB}$ \\
$\gamma_{m_{1}}$ & 1.0 & $\delta_{m_{1}}$ & 0.2 \\
$\kappa_{m_{1}}$ & 1.0 & $\sigma_{m_{1}}$ & 0.2 \\
$\gamma_{m_{2}}$ & 0.8 & $\delta_{m_{2}}$ & 0.5 \\
$\kappa_{m_{2}}$ & 0.8 & $\sigma_{m_{2}}$ & 0.5 \\
$\gamma_{m_{3}}$ & 1.2 & $\delta_{m_{3}}$ & 0.3 \\
$\kappa_{m_{3}}$ & 1.2 & $\sigma_{m_{3}}$ & 0.3 \\
$n_{s}$ & $100 \mathrm{users}$ & $P_{m}$ & var \\
$B\left(e_{i j}\right)$ & $10000 \mathrm{Mbps}$ & $B\left(v, v^{\prime}\right)$ & $30 \mathrm{Mbps}$ \\
$t_{e_{i j}}$ & $15 \mathrm{~ms}$ & $T_{s}$ & $50 \mathrm{~ms}$ \\
\hline
\end{tabular}

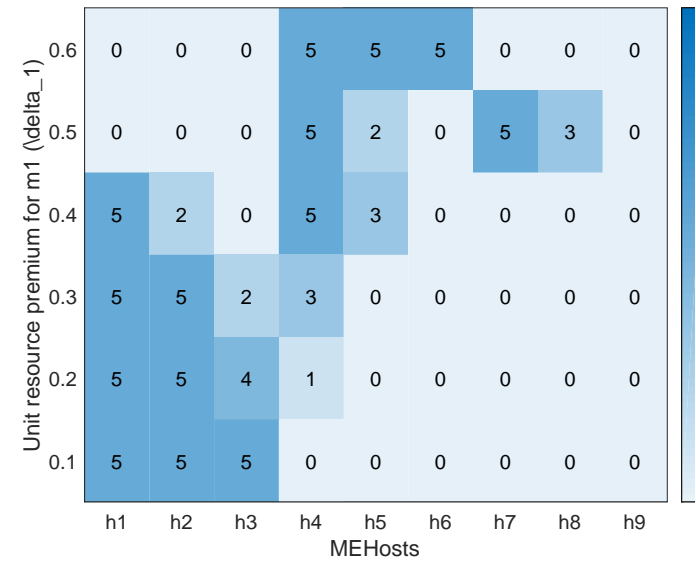

(a) $P_{m_{1}}=0.5$ and $P_{m_{2}}=P_{m_{3}}=0.25$

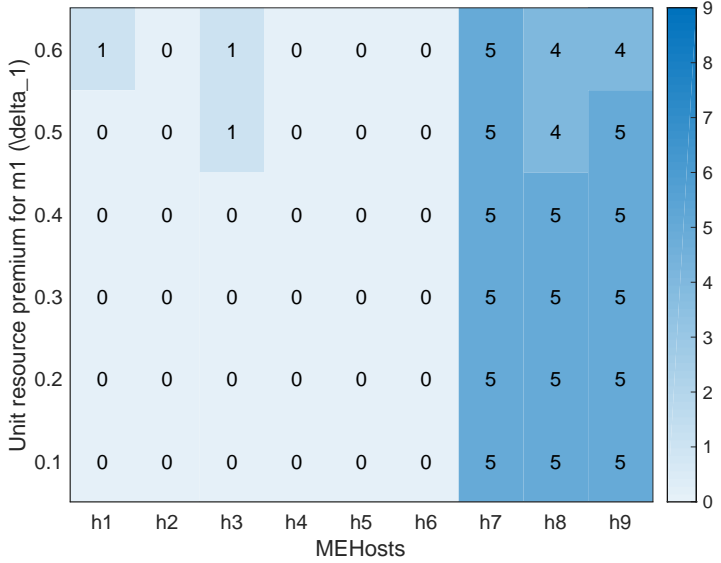

(b) $P_{m_{1}}=P_{m_{2}}=0.25$ and $P_{m_{3}}=0.5$

Figure 6.2: Placement results of 3 service chains consisting of 15 MEApps in all

\subsubsection{Placement trends with changing unit resource pre- mium}

The placement decision changes by the increase of $\delta_{m_{1}}$ under different user distributions are shown in Figure 6.2, where $\delta_{m_{1}}$, the unit resource premium payable 
to the MECSP for hosting MEApps for others, increases from 0.1 to 0.6. For comparison, in Figure 6.2(a), most users are from $m_{1}$. There is $P_{m_{1}}=0.5$ and $P_{m_{2}}=P_{m_{3}}=0.25$. Meanwhile, in Figure 6.2(b), most users subscribe services from $m_{3}$ as $P_{m_{1}}=P_{m_{1}}=0.25$ and $P_{m_{3}}=0.5$.

From the results of the two scenarios, we learn that the MEHosts with lower combination of unit resource base price $\left(\gamma_{m}\right)$ and unit resource premiums $\left(\delta_{m}\right)$ will be selected first. The MEHosts of the MECSP will have more weight upon consideration if there are more users from that MECSP.
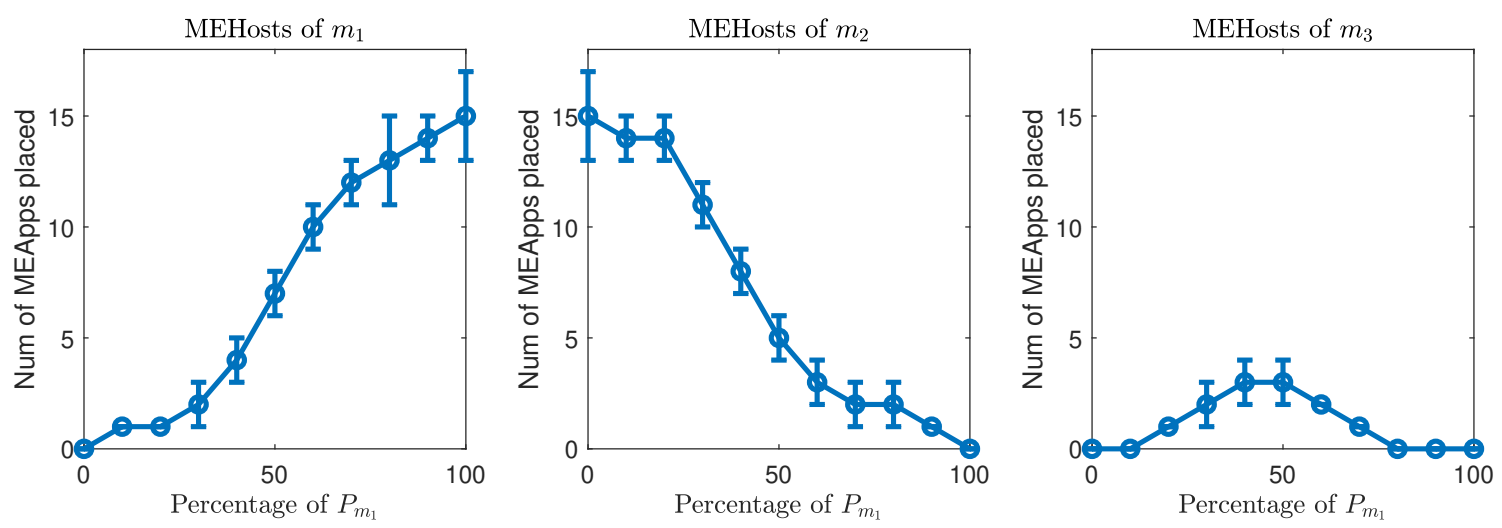

Figure 6.3: Numbers of MEApps placed on the 3 MEHosts with different percentages of users in the network with $95 \%$ confidence level

\subsubsection{Placement trends with changing user distribution}

To further demonstrate the impact from the distribution of the users, we simulate various scenarios with different percentages of users for $m_{1}$ and $m_{2}$, while there is no user for $m_{3}$. Users of $m_{1}$ increase from $0 \%$ to $100 \%$, while those of $m_{2}$ decrease from $100 \%$ to $0 \%$.

The results in Figure 6.3 have shown the trends of MEApps migrating to MEHosts owned by the MECSP that has more active users to avoid premiums charged by other MECSPs. However, resource sharing still takes place ( $m_{3}$ hosting MEApps for $m_{1}$ 
and $m_{2}$ ) when needed for better latency results and service quality.

\subsection{EdgeChain Design and Implementation}

In this section, we introduce the design and implementation of EdgeChain, a blockchain-based system that integrates with the existing MEC architecture for $M E C$ $S P s$ and the scheduler of $M E A V$. Blockchain is used in the system in the following way:

- The blockchain acts as a public ledger that stores all useful information and transactions made during the placement process. Exposure of the information would help the placement algorithm make optimized decisions considering the global resource demand and allocation. The blockchain enables such centralized resource information, in a decentralized implementation. As a public ledger applying proof-of-work verifications, the blockchain makes it nearly impossible to tamper the history stored in the blockchain.

- Due to its complexity, the EdgeChain optimization algorithm will be executed by a trusted third-party node with sufficient computing power. This node will ensure the neutrality of the placement decisions as it is independent from all MECSPs and MEAVs. The output of the algorithm will become the reference of $M E A V s$ to make placement decisions.

- Although the trusted node gives placement suggestions, the $M E A V s$ still can make placement decisions as they are the actual consumer of MEC resources. If $M E A V s$ decide to take suggestions made by the trusted node, the commercial transactions will be recorded by the blockchain.

- All other mining nodes participating in the blockchain will take the role to record 
the transactions on the ledger. If the placement decisions made by $M E A V s$ are different from the suggestions made by the trusted node, the transaction request will be rejected by the mining nodes to ensure the neutrality of the placement decision gets enforced.

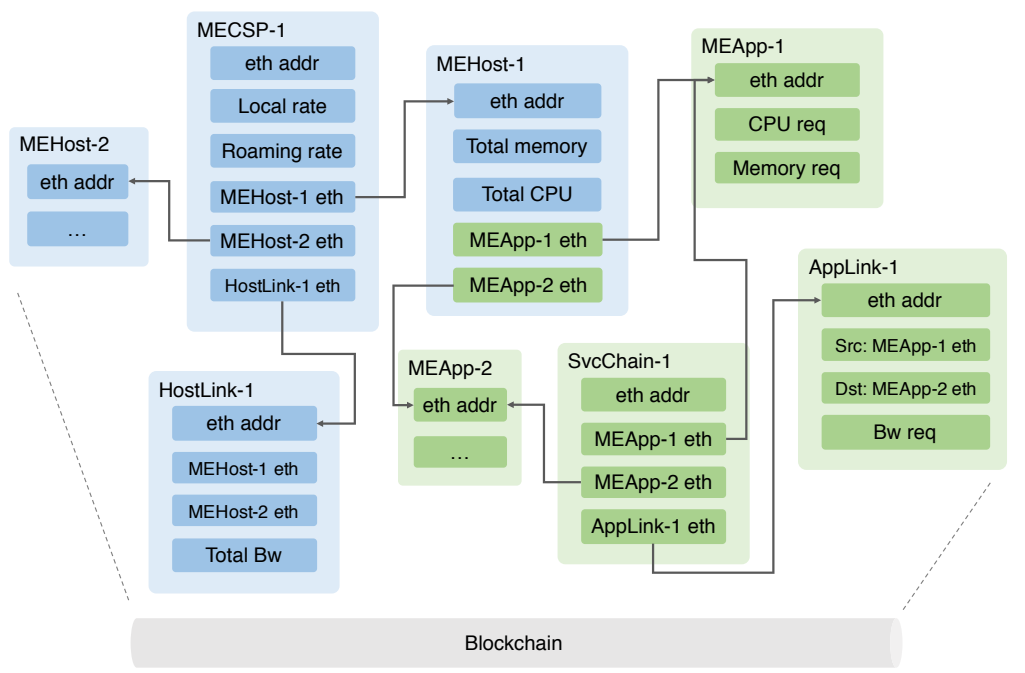

Figure 6.4: Data entities and their relationship used by and stored in EdgeChain, including MECSPs, MEHosts, HostLinks, SvcChains, MEApps, and AppLinks.

\subsubsection{Data Entities}

As Fig. 6.4 shows, there exist 6 types of data entities on the blockchain and they are related to each other to represent the status of MEHosts and placement decision of running MEApps. The descriptions of these data entities are illustrated below. Each data entity record has a unique Ethereum address for other to locate it on the blockchain. All types of data entities can be created, updated and deleted, while the blockchain will keep the audit trail of every change. 


\section{MECSP}

When a MECSP record is registered to EdgeChain, a record of this MECSP is added with the Ethereum addresses pointing to the records of all its eligible MEHosts and HostLinks. A MECSP record is updated whenever there is change to any MEHost or HostLink.

\section{MEHost}

A MEHost record registers under an existing MECSP to the blockchain. In a record, the vCPU and memory capabilities can be found, along with the Ethereum addresses pointing to the records of all MEApps placed onto it.

\section{HostLink}

Similar to MEHosts, a HostLink is under a registered MECSP, which contains the two MEHosts it connects, and the bandwidth of the HostLink.

\section{SvcChain}

A service chain is registered by a user to the blockchain to reflect the resource consumption of a chained service, including that from MEApps and the corresponding AppLinks. The service chain can have MEApps from multiple MEAVs.

\section{MEApp}

A $M E A V$ will submit a record of a MEApp whenever it needs to spin up one. A record stores the vCPU, memory usage of the MEApp. 


\section{AppLink}

AppLinks describe chained relationship between two MEApps. The source and destination MEApps are stored in an AppLink record, as well as network bandwidth requirement of this link.

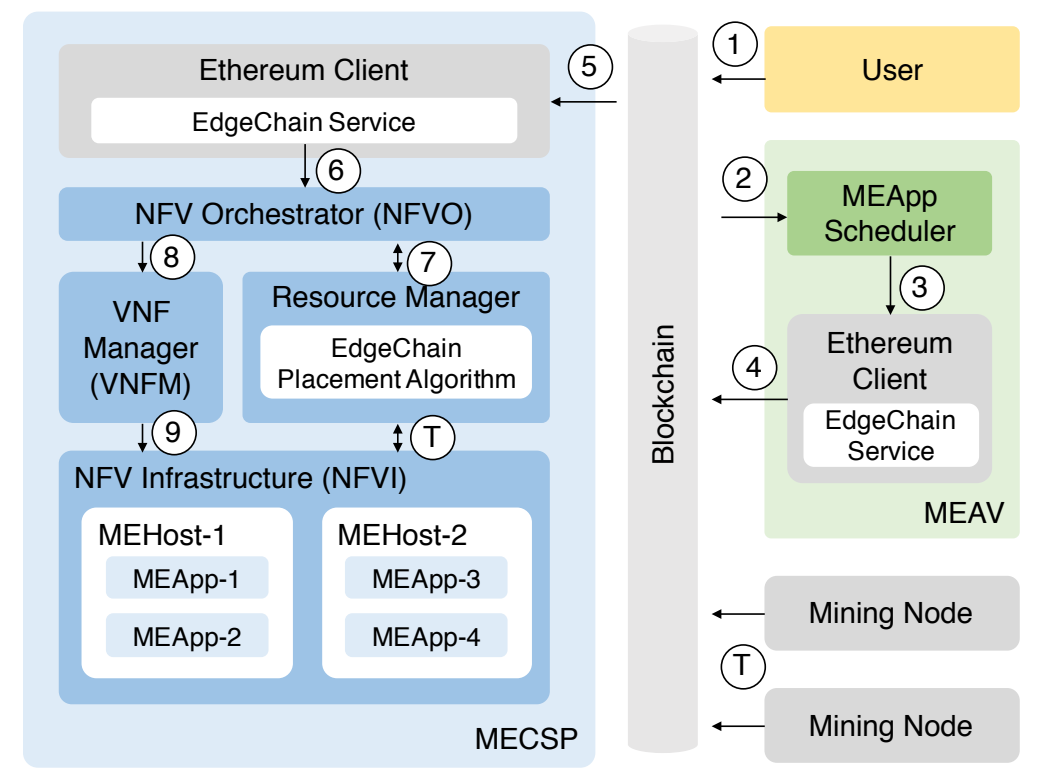

Figure 6.5: Typical work flow of EdgeChain. MECSPs, MEAVs, and mining nodes participate in the process. Steps of the work flow are marked by circled numbers and alphabets with details documented in Section 6.5.2.

\subsubsection{EdgeChain Work Flow}

A typical EdgeChain work flow can be demonstrated by Fig. 6.5, where there are three parties participating in the entire process: MECSPs, MEAVs, and mining nodes. We use circled numbers and alphabets to define the work flow in sequence.

(1) A user requests a service chain from the blockchain. Such requests will be sent to the blockchain every time a user requests a service chain.

(2) The request for the service chain is recorded. When the request is synced to the mining nodes, it will be broken into requests for MEApps. The mining nodes will 
run the logic to break down the service chain creation request. Then the requests for MEApps are propagated to all corresponding MEAVs.

(3) Based on its user demand, the MEApp Scheduler decides to create a new instance of MEApp and pass the request to the Ethereum client of the MEAV.

(4) The Ethereum client running the EdgeChain service sends the request to the blockchain, creating records for the request of placing a new MEApp.

(5) The request of creating a new MEApp arrives at a MECSP through its Ethereum client.

(6) For every MECSP, the Ethereum client requests the NFV Orchestrator (NFVO) to call the EdgeChain placement on the trusted node for the decision of the placement.

(7) The NFVO calls the trusted node to run EdgeChain algorithm for the placement decision. Note that the decision can be a hash representing any MEHost within the entire MEC network. If the result points to a MEHost which does not belong to the current MECSP, then no actual placement will be done. Instead, only the result along with the algorithm's hash will be returned to the Ethereum client for verification.

(8) If the result points to a MEHost of the current MECSP, then the NFVO will sends the request to place the MEApp to the VNF Manager (VNFM). Also, a transaction shown in Fig. 6.6 will be posted to the blockchain to record that placement actually occurs.

(9) The VNFM sends the request to the NFV Infrastructure (NFVI) deploy the MEAPP onto the target MEHost. 
(T) The mining nodes periodically perform the mining process to verify the blockchain, as well as earning Ethers for requesting placement services. Meanwhile, the resource manager periodically synchronizes with the NFVI for the upto-date resource usage and availability, and then posts the updated information to the blockchain.

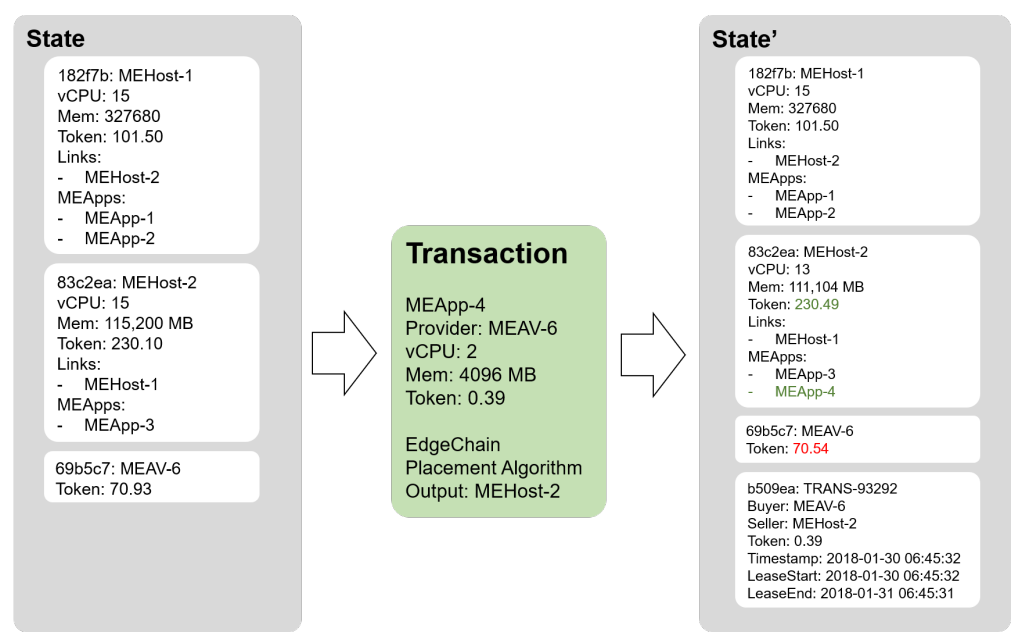

Figure 6.6: A placement transaction in EdgeChain. A state transition happens upon a transaction. As this figure shows, $M E A p p-4$ owned by $M E A V-6$ is to be placed with the requirement of $2 \mathrm{vCPUs}$ and $4096 \mathrm{MB}$ of memory. The input of the EdgeChain placement algorithm is the current state of the two MEHosts and MEAV-6. The result is to place MEApp-4 onto MEHost-2. After the transaction is accepted, the resources taken by MEApp-4 are deducted from the remaining resources of MEHost-2. $M E A V-6$ will pay tokens as fees to MEHost-2 for hosting the service.

\subsection{Chapter Summary}

In this chapter, we have presented the architecture and the algorithm for mobile edge applications placement with multiple mobile edge computing service providers. Future work implementing a blockchain-based system for collaboration of multiple vendor for resource sharing with the EdgeChain algorithm. 


\section{Chapter 7}

\section{Conclusions and Future Work}

\subsection{Conclusions}

In this dissertation, we have studied the security and availability of application offloading in cloud computing and MEC environments. From the cloud to the mobile edge, we have done an thorough literature survey of existing security issues, leading to the work of modeling the vulnerabilities in applications offloaded to the cloud. With the awareness of the risk for an offloaded application going down, we have presented a stochastic-programming-based model and a heuristic algorithm called EdgePlace, to leverage affinity and anti-affinity host placement rules for higher availability and lower cost. To increase the availability at the network edge, we have proposed IoT-B\&B to enable resource sharing of physical CPE nodes, in order to leverage unused resources at the network edge and to share them with users across the network edge. To further expand resources, we have explored resource sharing among SPs with EdgeChain, a model for making fair MEApps placement decisions for multiple SPs and a heuristic placement algorithm for MEApps across different SPs.

With the models and algorithms we have proposed, our conclusion is first around the evaluation of the vulnerability: building a graph of object dependencies can help 
identify the vulnerability of objects, and therefore help with the decision of keeping sensitive object running locally on the user's own mobile device instead of the cloud. By exercising the availability-ware mobile edge placement, we conclude the effectiveness of improving application availability by enforcing the placement policies. The IoT-B\&B and EdgeChain algorithm further concludes the potential resources and their values hidden at the CPE nodes and unused mobile edge hosts.

\subsection{Future work}

Regarding the application evaluation, our future work will first focus on quantifying the cloud-originated vulnerabilities. The calculation of propagated vulnerabilities depends on the cloud-originated vulnerabilities. Thus it directly affects accuracy of the ODG-based system. Like some intrusion detection systems (IDS), we can employ machine learning technology to find features that could impact cloud-originated vulnerabilities. Alternatively, these values can be retrieved and quantified from trusted parties, user reviews, and selected security analysis tools. Another item in the future work list is to remove the assumption that vulnerability propagation events are independent, as it is possible some vulnerability propagations are correlated because they share similar features. Besides, we need an optimization model to determine the objects to be offloaded once all vulnerabilities have been estimated. The model outputs the optimal offloading results and is responsible for all consequent communication costs and delay.

For the mobile edge application placement, the future work is to consider mobile edge applications with combinations of different NFs and with service chaining. Our current model has the assumption that all VMs are of the same type of a VNF. This assumption can be removed with extra work on making placement decisions on different types of VNFs. Making better use of the network edge is an interesting topic 
and has massive potential. Also, the mobility of the cloud needs to be considered as at the mobile edge, base stations along with the compute hosts can become mobile and loaded onto vehicles.

The IoT-B\&B model architecture can be strengthened by first exploring the availability to factor in the service up and down of B\&B nodes. This dissertation has used a constant factor to model the backup VNF instances. The modeling can be improved so that it is closer to the real-world scenario. More factors should be considered which impact the deployment placement besides vCPUs, memory and network bandwidth. Also, consider detailed factors that can indirectly impact the cost and the core network delay of the remote cloud. Models and algorithms for different combinations of VNFs need to be developed for more complex use cases with a combination of VNFs integrated together providing solution-level functionalities.

Regarding the EdgeChain framework, We can consider using a permissioned blockchain which uses Proof of Stake (PoS) instead of Proof of Work (PoW). This way, no mining nodes are needed and SPs can build a certain level of trust with each other to reduce cost and to increase the efficiency of the blockchain. Also, the resource allocation algorithm does not have to be executed on the blockchain itself as it would take too much computing power and become too expensive. Instead, the hash of the algorithm can be stored on the blockchain and node from SPs can run the resource allocation algorithm. Only the integrity of the algorithm and the results will be verified by the hash. 


\section{List of References}

[1] M. Satyanarayanan, P. Bahl, R. Caceres, and N. Davies, "The case for vm-based cloudlets in mobile computing," IEEE Pervasive Computing, vol. 8, pp. 14-23, Oct 2009 .

[2] B.-G. Chun and P. Maniatis, "Augmented smartphone applications through clone cloud execution," in Proceedings of the 12th Conference on Hot Topics in Operating Systems, HotOS'09, (Monte Verita, Switzerland), pp. 8-8, USENIX Association, May 2009.

[3] B.-G. Chun, S. Ihm, P. Maniatis, M. Naik, and A. Patti, "Clonecloud: Elastic execution between mobile device and cloud," in Proceedings of the Sixth Conference on Computer Systems, EuroSys '11, (Salzburg, Austria), pp. 301-314, ACM, 2011.

[4] S. Kosta, A. Aucinas, P. Hui, R. Mortier, and X. Zhang, "Thinkair: Dynamic resource allocation and parallel execution in the cloud for mobile code offloading," in 2012 Proceedings IEEE INFOCOM, pp. 945-953, March 2012.

[5] M. Satyanarayanan, "The Emergence of Edge Computing," Computer, vol. 50, pp. 30-39, Jan 2017.

[6] P. Garcia Lopez, A. Montresor, D. Epema, A. Datta, T. Higashino, A. Iamnitchi, M. Barcellos, P. Felber, and E. Riviere, "Edge-centric computing: Vision and challenges," SIGCOMM Comput. Commun. Rev., vol. 45, pp. 37-42, Sept. 2015.

[7] Y. C. Hu, M. Patel, D. Sabella, N. Sprecher, and V. Young, "Mobile edge computinga key technology towards 5g," ETSI White Paper, vol. 11, 2015.

[8] W. Jaquith, "The New Trend of Regional Data Centers." https://usopendata.org/2015/03/25/rdc/. [Online; accessed Mar-04-2017]. 
[9] F. Bonomi, R. Milito, J. Zhu, and S. Addepalli, "Fog computing and its role in the internet of things," in Proceedings of the First Edition of the MCC Workshop on Mobile Cloud Computing, MCC '12, (Helsinki, Finland), pp. 13-16, ACM, 2012.

[10] J. Martins, M. Ahmed, C. Raiciu, V. Olteanu, M. Honda, R. Bifulco, and F. Huici, "ClickOS and the art of network function virtualization," in 11th USENIX Symposium on Networked Systems Design and Implementation (NSDI 14), (Seattle, WA), pp. 459-473, USENIX Association, 2014.

[11] B. Han, V. Gopalakrishnan, L. Ji, and S. Lee, "Network function virtualization: Challenges and opportunities for innovations," IEEE Communications Magazine, vol. 53, pp. 90-97, Feb 2015.

[12] S. Beereddy and K. Sirupa, "NFV use case - Delivering virtual CPE with multivendor VNF orchestration," in 2015 IEEE Conference on Network Function Virtualization and Software Defined Network (NFV-SDN), (San Francisco, CA, USA), pp. 25-27, Nov 2015.

[13] J. Soares, M. Dias, J. Carapinha, B. Parreira, and S. Sargento, "Cloud4nfv: A platform for virtual network functions," in 2014 IEEE 3rd International Conference on Cloud Networking (CloudNet), (Luxembourg), pp. 288-293, Oct 2014.

[14] T. Taleb, M. Corici, C. Parada, A. Jamakovic, S. Ruffino, G. Karagiannis, and T. Magedanz, "EASE: EPC as a service to ease mobile core network deployment over cloud," IEEE Network, vol. 29, pp. 78-88, March 2015.

[15] C. Cachin, I. Keidar, and A. Shraer, "Trusting the cloud," SIGACT News, vol. 40, pp. 81-86, June 2009.

[16] T. Ristenpart, E. Tromer, H. Shacham, and S. Savage, "Hey, you, get off of my cloud: Exploring information leakage in third-party compute clouds," in Proceedings of the 16th ACM Conference on Computer and Communications Security, CCS '09, (Chicago, Illinois, USA), pp. 199-212, ACM, 2009.

[17] Y. Chen and R. Sion, "On securing untrusted clouds with cryptography," in Proceedings of the 9th Annual ACM Workshop on Privacy in the Electronic Society, WPES '10, (Chicago, Illinois, USA), pp. 109-114, ACM, 2010.

[18] H. Liu, F. Eldarrat, H. Alqahtani, A. Reznik, X. de Foy, and Y. Zhang, "Mobile edge cloud system: Architectures, challenges, and approaches," IEEE Systems Journal, vol. PP, no. 99, pp. 1-14, 2017. 
[19] M. T. Beck, M. Werner, S. Feld, and S. Schimper, "Mobile edge computing: A taxonomy," in Proc. of the Sixth International Conference on Advances in Future Internet, Citeseer, 2014.

[20] J. M. Halpern and C. Pignataro, "Service Function Chaining (SFC) Architecture." RFC 7665, Oct. 2015.

[21] M. Jammal, A. Kanso, and A. Shami, "High availability-aware optimization digest for applications deployment in cloud," in 2015 IEEE International Conference on Communications (ICC), (London, UK), pp. 6822-6828, June 2015.

[22] S. Oechsner and A. Ripke, "Flexible support of VNF placement functions in OpenStack," in Proceedings of the 2015 1st IEEE Conference on Network Softwarization (NetSoft), (London, UK), pp. 1-6, April 2015.

[23] N. Herbaut, D. Negru, G. Xilouris, and Y. Chen, "Migrating to a nfv-based home gateway: Introducing a surrogate vnf approach," in 2015 6th International Conference on the Network of the Future (NOF), (Montreal, QC, Canada), pp. 1-7, Sept 2015.

[24] F. Bonomi, R. Milito, J. Zhu, and S. Addepalli, "Fog computing and its role in the internet of things," in Proceedings of the First Edition of the MCC Workshop on Mobile Cloud Computing, MCC '12, (Helsinki, Finland), pp. 13-16, ACM, 2012.

[25] F. Gens, "New IDC IT cloud services survey: Top benefits and challenges," IDC exchange, pp. 17-19, 2009.

[26] R. L. Krutz and R. D. Vines, Cloud security: A comprehensive guide to secure cloud computing. Wiley Publishing, 2010.

[27] R. Schaeffer, "National information assurance (IA) glossary," CNSS Secretariat, NSA, Ft. Meade, 2010.

[28] W.-M. Hu, "Lattice scheduling and covert channels," in Research in Security and Privacy, 1992. Proceedings., 1992 IEEE Computer Society Symposium on, (Oakland, CA, USA), pp. 52-61, IEEE, 1992.

[29] Y. Xu, M. Bailey, F. Jahanian, K. Joshi, M. Hiltunen, and R. Schlichting, "An exploration of 12 cache covert channels in virtualized environments," in Proceedings of the 3rd ACM Workshop on Cloud Computing Security Workshop, CCSW '11, (Chicago, Illinois, USA), pp. 29-40, ACM, 2011. 
[30] D. X. Song, D. Wagner, and X. Tian, "Timing analysis of keystrokes and timing attacks on ssh," in Proceedings of the 10th Conference on USENIX Security Symposium - Volume 10, SSYM'01, (Washington, D.C., USA), USENIX Association, 2001.

[31] H. Liu, "A new form of dos attack in a cloud and its avoidance mechanism," in Proceedings of the 2010 ACM Workshop on Cloud Computing Security Workshop, CCSW'10, (Chicago, Illinois, USA), pp. 65-76, ACM, 2010.

[32] M. McIntosh and P. Austel, "XML signature element wrapping attacks and countermeasures," in Proceedings of the 2005 Workshop on Secure Web Services, SWS '05, (Fairfax, VA, USA), pp. 20-27, ACM, 2005.

[33] M. Christodorescu, R. Sailer, D. L. Schales, D. Sgandurra, and D. Zamboni, "Cloud security is not (just) virtualization security: A short paper," in Proceedings of the 2009 ACM Workshop on Cloud Computing Security, CCSW '09, (Chicago, Illinois, USA), pp. 97-102, ACM, 2009.

[34] R. Sandhu, R. Boppana, R. Krishnan, J. Reich, T. Wolff, and J. Zachry, "Towards a discipline of mission-aware cloud computing," in Proceedings of the 2010 ACM Workshop on Cloud Computing Security Workshop, CCSW '10, (Chicago, Illinois, USA), pp. 13-18, ACM, 2010.

[35] A. Aviram, S. Hu, B. Ford, and R. Gummadi, "Determinating timing channels in compute clouds," in Proceedings of the 2010 ACM Workshop on Cloud Computing Security Workshop, CCSW '10, (Chicago, Illinois, USA), pp. 103-108, ACM, 2010.

[36] H. Raj, R. Nathuji, A. Singh, and P. England, "Resource management for isolation enhanced cloud services," in Proceedings of the 2009 ACM Workshop on Cloud Computing Security, CCSW '09, (Chicago, Illinois, USA), pp. 77-84, ACM, 2009.

[37] B. C. Vattikonda, S. Das, and H. Shacham, "Eliminating fine grained timers in xen," in Proceedings of the 3rd ACM Workshop on Cloud Computing Security Workshop, CCSW '11, (Chicago, Illinois, USA), pp. 41-46, ACM, 2011.

[38] E. Keller, J. Szefer, J. Rexford, and R. B. Lee, "Nohype: Virtualized cloud infrastructure without the virtualization," in Proceedings of the 37th Annual International Symposium on Computer Architecture, ISCA '10, (Saint-Malo, France), pp. 350-361, ACM, 2010. 
[39] J. Du, X. Gu, and T. Yu, "On verifying stateful dataflow processing services in large-scale cloud systems," in Proceedings of the 17th ACM Conference on Computer and Communications Security, CCS '10, (Chicago, Illinois, USA), pp. 672-674, ACM, 2010.

[40] R. Chow, M. Jakobsson, R. Masuoka, J. Molina, Y. Niu, E. Shi, and Z. Song, "Authentication in the clouds: A framework and its application to mobile users," in Proceedings of the 2010 ACM Workshop on Cloud Computing Security Workshop, CCSW '10, (Chicago, Illinois, USA), pp. 1-6, ACM, 2010.

[41] G. Danezis and B. Livshits, "Towards ensuring client-side computational integrity," in Proceedings of the 3rd ACM Workshop on Cloud Computing Security Workshop, CCSW '11, (Chicago, Illinois, USA), pp. 125-130, ACM, 2011.

[42] K. Zhang, X. Zhou, Y. Chen, X. Wang, and Y. Ruan, "Sedic: Privacy-aware data intensive computing on hybrid clouds," in Proceedings of the 18th ACM Conference on Computer and Communications Security, CCS '11, (Chicago, Illinois, USA), pp. 515-526, ACM, 2011.

[43] S. Bugiel, S. Nürnberger, T. Pöppelmann, A.-R. Sadeghi, and T. Schneider, "AmazonIA: When elasticity snaps back," in Proceedings of the 18th ACM Conference on Computer and Communications Security, CCS '11, (Chicago, Illinois, USA), pp. 389-400, ACM, 2011.

[44] P. Saripalli and B. Walters, "QUIRC: A quantitative impact and risk assessment framework for cloud security," in 2010 IEEE 3rd International Conference on Cloud Computing, (Miami, FL, USA), pp. 280-288, July 2010.

[45] S. Bleikertz, M. Schunter, C. W. Probst, D. Pendarakis, and K. Eriksson, "Security audits of multi-tier virtual infrastructures in public infrastructure clouds," in Proceedings of the 2010 ACM Workshop on Cloud Computing Security Workshop, CCSW'10, (Chicago, Illinois, USA), pp. 93-102, ACM, 2010.

[46] J. Wei, X. Zhang, G. Ammons, V. Bala, and P. Ning, "Managing security of virtual machine images in a cloud environment," in Proceedings of the 2009 ACM Workshop on Cloud Computing Security, CCSW '09, (Chicago, Illinois, USA), pp. 91-96, ACM, 2009.

[47] X. Zhang, J. Schiffman, S. Gibbs, A. Kunjithapatham, and S. Jeong, "Securing elastic applications on mobile devices for cloud computing," in Proceedings of the 2009 ACM Workshop on Cloud Computing Security, CCSW '09, (Chicago, Illinois, USA), pp. 127-134, ACM, 2009. 
[48] H. Liang, D. Huang, L. X. Cai, X. Shen, and D. Peng, "Resource allocation for security services in mobile cloud computing," in 2011 IEEE Conference on Computer Communications Workshops (INFOCOM WKSHPS), (Shanghai, China), pp. 191-195, April 2011.

[49] W. Jia, H. Zhu, Z. Cao, L. Wei, and X. Lin, "SDSM: A secure data service mechanism in mobile cloud computing," in 2011 IEEE Conference on Computer Communications Workshops (INFOCOM WKSHPS), (Shanghai, China), pp. 1060-1065, April 2011.

[50] B.-G. Chun and P. Maniatis, "Dynamically partitioning applications between weak devices and clouds," in Proceedings of the 1st ACM Workshop on Mobile Cloud Computing 83\#38; Services: Social Networks and Beyond, MCS '10, (San Francisco, California), pp. 7:1-7:5, ACM, 2010.

[51] E. Cuervo, A. Balasubramanian, D.-k. Cho, A. Wolman, S. Saroiu, R. Chandra, and P. Bahl, "Maui: Making smartphones last longer with code offload," in Proceedings of the 8th International Conference on Mobile Systems, Applications, and Services, MobiSys '10, (San Francisco, California, USA), pp. 49-62, ACM, 2010.

[52] Z. Xiao, W. Song, and Q. Chen, "Dynamic resource allocation using virtual machines for cloud computing environment," IEEE Transactions on Parallel and Distributed Systems, vol. 24, pp. 1107-1117, June 2013.

[53] Y. Wen, W. Zhang, and H. Luo, "Energy-optimal mobile application execution: Taming resource-poor mobile devices with cloud clones," in 2012 Proceedings IEEE INFOCOM, (Orlando, FL, USA), pp. 2716-2720, March 2012.

[54] G. Portokalidis, P. Homburg, K. Anagnostakis, and H. Bos, "Paranoid android: Versatile protection for smartphones," in Proceedings of the 26th Annual Computer Security Applications Conference, ACSAC '10, (Austin, Texas, USA), pp. 347-356, ACM, 2010.

[55] G. C. Hunt and M. L. Scott, "The coign automatic distributed partitioning system," in Proceedings of the Third Symposium on Operating Systems Design and Implementation, OSDI '99, (New Orleans, Louisiana, USA), pp. 187-200, USENIX Association, 1999.

[56] E. Tilevich and Y. Smaragdakis, "J-orchestra: Automatic java application partitioning," in ECOOP 2002 - Object-Oriented Programming (B. Magnusson, ed.), (Berlin, Heidelberg), pp. 178-204, Springer Berlin Heidelberg, 2002. 
[57] "Java remote method invocation."

[58] ETSI Group Specification, "Mobile Edge Computing (MEC); Framework and Reference Architecture," ETSI GS MEC 003, V1.1.1 (2016-03).

[59] ETSI Group Specification, "Network Function Virtualisation(NFV); Architectural Framework," ETSI GS NFV 002, V1.1.1 (2013-10).

[60] R. Mijumbi, J. Serrat, J. L. Gorricho, N. Bouten, F. D. Turck, and R. Boutaba, "Network function virtualization: State-of-the-art and research challenges," IEEE Communications Surveys Tutorials, vol. 18, pp. 236-262, Firstquarter 2016.

[61] P. Veitch, M. J. McGrath, and V. Bayon, "An instrumentation and analytics framework for optimal and robust nfv deployment," IEEE Communications Magazine, vol. 53, pp. 126-133, Feb 2015.

[62] A. Basta, W. Kellerer, M. Hoffmann, H. J. Morper, and K. Hoffmann, "Applying $\mathrm{nfv}$ and sdn to lte mobile core gateways, the functions placement problem," in Proceedings of the 4th Workshop on All Things Cellular: Operations, Applications, E\#\#38; Challenges, AllThingsCellular '14, (Chicago, Illinois, USA), pp. 33-38, ACM, 2014.

[63] Z. Usmani and S. Singh, "A survey of virtual machine placement techniques in a cloud data center," Procedia Comput. Sci., vol. 78, pp. 491-498, Mar. 2016.

[64] U. Bellur, C. S. Rao, and S. D. M. Kumar, "Optimal placement algorithms for virtual machines," CoRR, vol. abs/1011.5064, 2010.

[65] A. Khosravi, S. K. Garg, and R. Buyya, "Energy and carbon-efficient placement of virtual machines in distributed cloud data centers," in Euro-Par 2013 Parallel Processing - 19th International Conference, Aachen, Germany, August 26-30, 2013. Proceedings, pp. 317-328, 2013.

[66] J. Zhu and C. Huang, "A universal protocol mechanism for network function virtualization and application-centric traffic steering," in IEEE Conference on Standards for Communications and Networking, CSCN 2015, Tokyo, Japan, October 28-30, 2015, pp. 257-262, 2015.

[67] S. Clayman, E. Maini, A. Galis, A. Manzalini, and N. Mazzocca, "The dynamic placement of virtual network functions," in 2014 IEEE Network Operations and 
Management Symposium, NOMS 2014, Krakow, Poland, May 5-9, 2014, pp. 19, 2014.

[68] H. Moens and F. D. Turck, "VNF-P: A model for efficient placement of virtualized network functions," in 10th International Conference on Network and Service Management, CNSM 2014 and Workshop, Rio de Janeiro, Brazil, November 17-21, 2014, pp. 418-423, 2014.

[69] G. Brown, "Service chaining in carrier networks," Heavy Reading, Feb 2015.

[70] P. Quinn and J. Guichard, "Service function chaining: Creating a service plane via network service headers," Computer, vol. 47, pp. 38-44, Nov 2014.

[71] J. Blendin, J. Rckert, N. Leymann, G. Schyguda, and D. Hausheer, "Position paper: Software-defined network service chaining," in 2014 Third European Workshop on Software Defined Networks, (London, UK), pp. 109-114, Sept 2014.

[72] M. T. Beck and J. F. Botero, "Coordinated allocation of service function chains," in 2015 IEEE Global Communications Conference (GLOBECOM), (San Diego, CA, USA), pp. 1-6, Dec 2015.

[73] OpenStack. http://www.openstack.org/, 2017. [Online; accessed Nov-27-2017].

[74] VMWare. http://www.vmware.com/, 2017. [Online; accessed Nov-27-2017].

[75] ETSI Industry Specification Group (ISG) NFV, "ETSI GS NFV 002 V1.2.1: Network Functions Virtualisation (NFV): Architectural Framework," 2014.

[76] W. Shen, M. Yoshida, T. Kawabata, K. Minato, and W. Imajuku, "vconductor: An nfv management solution for realizing end-to-end virtual network services," in The 16th Asia-Pacific Network Operations and Management Symposium, (Hsinchu, Taiwan), pp. 1-6, Sept 2014.

[77] H. Zhu, C. Huang, and J. Yan, "Vulnerability evaluation for securely offloading mobile apps in the cloud," in 2013 IEEE 2nd International Conference on Cloud Networking (CloudNet), (San Francisco, CA, USA), pp. 108-116, Nov 2013.

[78] L. M. Vaquero and L. Rodero-Merino, "Finding your way in the fog: Towards a comprehensive definition of fog computing," ACM SIGCOMM Computer Communication Review, vol. 44, pp. 27-32, Oct. 2014. 
[79] I. Stojmenovic and S. Wen, "The fog computing paradigm: Scenarios and security issues," in 2014 Federated Conference on Computer Science and Information Systems, (Warsaw, Poland), pp. 1-8, Sept 2014.

[80] K. Yang, K. Zhang, J. Ren, and X. Shen, "Security and privacy in mobile crowdsourcing networks: challenges and opportunities," IEEE Communications Magazine, vol. 53, pp. 75-81, August 2015.

[81] A. Manzalini, R. Minerva, F. Callegati, W. Cerroni, and A. Campi, "Clouds of virtual machines in edge networks," IEEE Communications Magazine, vol. 51, pp. 63-70, July 2013.

[82] F. Callegati, W. Cerroni, C. Contoli, and G. Santandrea, "Dynamic chaining of virtual network functions in cloud-based edge networks," in Proceedings of the 2015 1st IEEE Conference on Network Softwarization (NetSoft), (London, UK), pp. 1-5, April 2015.

[83] A. Lombardo, A. Manzalini, G. Schembra, G. Faraci, C. Rametta, and V. Riccobene, "An open framework to enable netfate (network functions at the edge)," in Proceedings of the 2015 1st IEEE Conference on Network Softwarization (NetSoft), (London, UK), pp. 1-6, April 2015.

[84] W. John, K. Pentikousis, G. Agapiou, E. Jacob, M. Kind, A. Manzalini, F. Risso, D. Staessens, R. Steinert, and C. Meirosu, "Research directions in network service chaining," in 2013 IEEE SDN for Future Networks and Services (SDN4FNS), (Trento, Italy), pp. 1-7, Nov 2013.

[85] P. Quinn and T. Nadeau, "Problem statement for service function chaining," RFC 7498, 2015.

[86] H. Zhu and C. Huang, "Availability-aware mobile edge application placement in 5g networks," in GLOBECOM 2017 - 2017 IEEE Global Communications Conference, (Singapore), pp. 1-6, Dec 2017.

[87] Z. Xiong, S. Feng, D. Niyato, P. Wang, and Z. Han, "Edge computing resource management and pricing for mobile blockchain," arXiv preprint arXiv:1710.0156r, 2017.

[88] A. Stanciu, "Blockchain based distributed control system for edge computing," in 2017 21st International Conference on Control Systems and Computer Science (CSCS), (Bucharest, Romania), pp. 667-671, May 2017. 
[89] M. Samaniego and R. Deters, "Blockchain as a service for iot," in 2016 IEEE International Conference on Internet of Things (iThings) and IEEE Green Computing and Communications (GreenCom) and IEEE Cyber, Physical and Social Computing (CPSCom) and IEEE Smart Data (SmartData), (Chengdu, China), pp. 433-436, Dec 2016.

[90] S. Nakamoto, "Bitcoin: A peer-to-peer electronic cash system," 2008.

[91] D. Bayer, S. Haber, and W. S. Stornetta, Improving the Efficiency and Reliability of Digital Time-Stamping, pp. 329-334. Springer, 1993.

[92] G. Wood, "Ethereum: A secure decentralised generalised transaction ledger," Ethereum Project Yellow Paper, vol. 151, 2014.

[93] P. Resnick, K. Kuwabara, R. Zeckhauser, and E. Friedman, "Reputation systems," Communications of the ACM, vol. 43, no. 12, pp. 45-48, 2000.

[94] R. L. Biddle, E. Tempero, and N. Wellington, Understanding OOP language support for reusability. Department of Computer Science, Victoria University of Wellington, 1995.

[95] Y. Khmelevsky and V. Voytenko, "Cloud computing infrastructure prototype for university education and research," in Proceedings of the 15th Western Canadian Conference on Computing Education, WCCCE' 10, (Kelowna, British Columbia, Canada), pp. 8:1-8:5, ACM, 2010.

[96] S. Buchegger and J.-Y. Le Boudec, "A robust reputation system for peer-topeer and mobile ad-hoc networks," in Proceedings of the 2nd Workshop on Economics of Peer-to-peer Systems, no. LCA-CONF-2004-009, 2004.

[97] J. Golbeck and J. Hendler, "Reputation Network Analysis for Email Filtering," in Proceeding of Conference on Email and Anti-Spam (CEAS), (Mountain View, CA, USA), July 2004.

[98] P. Resnick and R. Zeckhauser, "Trust among strangers in internet transactions: Empirical analysis of ebay's reputation system," in The Economics of the Internet and E-commerce, pp. 127-157, Emerald Group Publishing Limited, 2002.

[99] J. D. Work, A. Blue, and R. Hoffman, "Method and system for reputation evaluation of online users in a social networking scheme," August 2011. US Patent 8,010,460. 
[100] S. M. Ross, Introduction to probability models. Academic press, 2014.

[101] L. Rosenberg, "Algorithm for finding minimal cut sets in a fault tree," Reliability Engineering 8 System Safety, vol. 53, no. 1, pp. 67-71, 1996.

[102] G. Kiczales and E. Hilsdale, "Aspect-oriented programming," in Proceedings of the 8th European Software Engineering Conference Held Jointly with 9th ACM SIGSOFT International Symposium on Foundations of Software Engineering, ESEC/FSE-9, (Vienna, Austria), pp. 313-, ACM, 2001.

[103] L. Zhang, B. Tiwana, Z. Qian, Z. Wang, R. P. Dick, Z. M. Mao, and L. Yang, "Accurate online power estimation and automatic battery behavior based power model generation for smartphones," in Proceedings of the eighth IEEE/ACM/IFIP international conference on Hardware/software codesign and system synthesis, (Scottsdale, AZ, USA), pp. 105-114, ACM, Oct 2010.

[104] S. E. Kevin Brown, Wendy Torell, "Edge computing needs reliability." http://www.datacenterdynamics.com/content-tracks/power-cooling/edgecomputing-needs-reliability/97587.fullarticle. [Online; accessed Mar-24-2017].

[105] U. Vyas, "Designing Your First Cloud with OpenStack," in Applied OpenStack Design Patterns, pp. 1-17, Springer, 2016.

[106] S. Lee, S. Pack, M.-K. Shin, and R. Browne, "Resource management in service chaining,"

[107] A. Cassen, "Keepalived: Health checking for lvs \& high availability," URL http://www.linuxvirtualserver.org, 2002.

[108] J. E. Beasley, "Stochastic programming." http://people.brunel.ac.uk/ mastjjb/jeb/or/sp.html, 2018. [Online; accessed May-8-2018].

[109] A. A. Gaivoronski, A. Lisser, R. Lopez, and H. Xu, "Knapsack problem with probability constraints," Journal of Global Optimization, vol. 49, no. 3, pp. 397413, 2011.

[110] E. Telecom, "White Paper: The Definitive Guide to vCPE." https://www. ecitele.com/media/1703/white-paper-the-definitive-guide-to-vcpe. pdf, 2017. [Online; accessed Nov-27-2017].

[111] K. Jang, J. Sherry, H. Ballani, and T. Moncaster, "Silo: Predictable message latency in the cloud," ACM SIGCOMM Computer Communication Review, vol. 45, no. 4, pp. 435-448, 2015. 
[112] OpenStack: Overcommitting CPU and RAM. https://docs.openstack.org/archdesign/design-compute/design-compute-overcommit.html, 2017. [Online; accessed Nov-27-2017].

[113] S. A. Baset, L. Wang, and C. Tang, "Towards an understanding of oversubscription in cloud," in Proceedings of the 2Nd USENIX Conference on Hot Topics in Management of Internet, Cloud, and Enterprise Networks and Services, HotICE'12, (San Jose, CA), pp. 7-7, USENIX Association, 2012.

[114] Amazon EC2. https://aws.amazon.com/ec2/, 2017. [Online; accessed Nov-272017].

[115] The CentOS Project. https://www.centos.org/, 2017. [Online; accessed Nov27-2017].

[116] OpenStack Kolla Project. https://wiki.openstack.org/wiki/Kolla/, 2017. [Online; accessed Nov-27-2017].

[117] A. N. Gorban, L. I. Pokidysheva, E. V. Smirnova, and T. A. Tyukina, "Law of the minimum paradoxes," Bulletin of Mathematical Biology, vol. 73, pp. 20132044, Nov 2010.

[118] W. Shi, J. Cao, Q. Zhang, Y. Li, and L. Xu, "Edge computing: Vision and challenges," IEEE Internet of Things Journal, vol. 3, pp. 637-646, Oct 2016.

[119] R. N. Calheiros, R. Ranjan, A. Beloglazov, C. A. F. De Rose, and R. Buyya, "Cloudsim: A toolkit for modeling and simulation of cloud computing environments and evaluation of resource provisioning algorithms," Softw. Pract. Exper., vol. 41, pp. 23-50, Jan. 2011.

[120] Bud Gerstman, "T table." http://www.sjsu.edu/faculty/gerstman/StatPrimer/ttable.pdf, 2018. [Online; accessed May-8-2018]. 


\section{Appendix A}

\section{Confidence interval}

A confidence interval (CI) gives an estimated range of values which is likely to include an unknown population parameter, the estimated range being calculated from a given set of sample data.

The purpose of taking a random sample from a population and computing a statistic, such as the mean from the data, is to approximate the mean of the population. How well the sample statistic estimates the underlying population value is always an issue. A confidence interval addresses this issue because it provides a range of values which is likely to contain the population parameter of interest.

Confidence intervals are constructed at a confidence level, such as $95 \%$, selected by the user. If the same population is sampled on numerous occasions and interval estimates are made on each occasion, the resulting intervals would bracket the true population parameter in approximately $95 \%$ of the cases. A confidence stated at a $(1-\alpha)$ level can be thought of as the inverse of a significance level, $\alpha$.

In our case, we have $n=5$ samples for each of the data point. The sample size is smaller than 20 and population standard deviation $\sigma$ is unknown. This is a typical case for using $\mathrm{T}$ distribution. In our case, we use two-tailed $\mathrm{T}$ distribution because the results in our analysis shall all be non-negative numbers. For samples with $\mathrm{T}$ 
distribution, a 100(1- $\alpha) \%$ confidence interval for the mean of a normal population is

$$
\hat{X} \pm \frac{t_{\alpha / 2} \sigma}{\sqrt{N}}
$$

To get the CI of the samples, we first make sure that the results are collected after the warm-up period ends. This allows the system to get into conditions that are typical of normal running conditions. Also, each sample value is independent to other samples. To ensure the independence of the samples, we get a new sample by giving a new run of the simulation and only read the sample after the system has been in a stable state. Figure shows an example of how our system warms up and eventually enters a stable state. For all examples we pick for CI, they are retrieved after the system is up for over 200 seconds to ensure the stability of the system and samples.

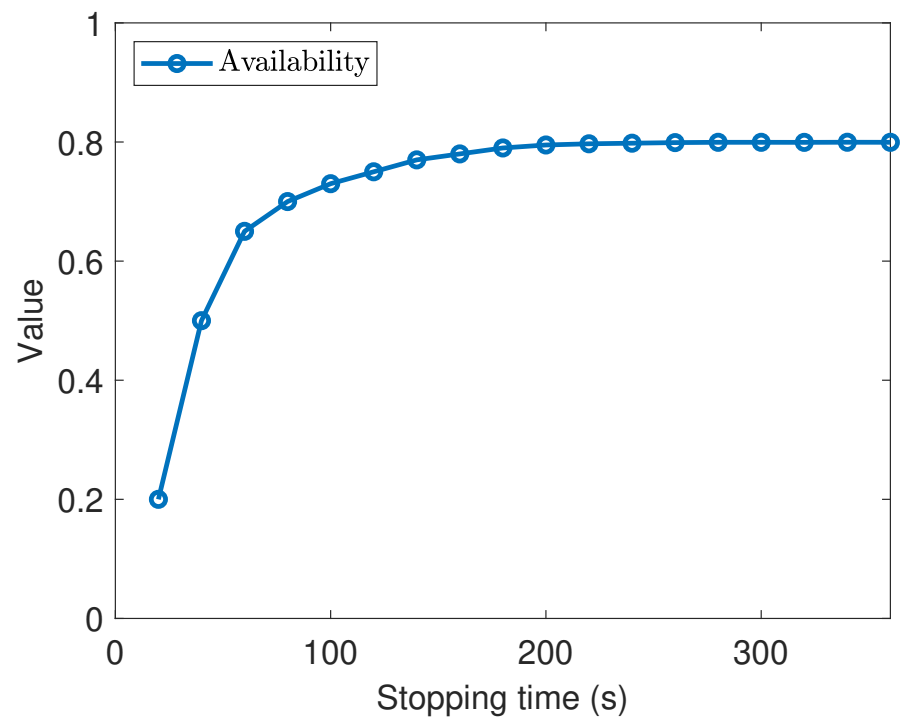

Figure A.1: Estimated availability values versus stopping time. For getting the example when the system is in stable state, the stopping time needs to be greater than 200 seconds 
Consider the following 5 samples of an availability value:

$$
[0.85293,0.85435,0.85710,0.85019,0.84986]
$$

where $\hat{X}$ is the sample mean, $t_{1-\alpha / 2}$ is the $1-\alpha / 2$ critical value of the $\mathrm{T}$ distribution which is found in the $T$ distribution table [120], $\sigma$ is the standard deviation of the samples, and $N$ is the sample size. To look up the $T$ distribution table, we also need the degree of freedom for our sample, denoted by $d f$, where $d f=n-1=4$ in our case.

Table A.1: T distribution table (one-tail, partial)

\begin{tabular}{l|l}
\hline df & $\alpha=\mathbf{0 . 0 2 5}$ \\
\hline 1 & 12.706 \\
2 & 4.303 \\
3 & 3.182 \\
$\mathbf{4}$ & $\mathbf{2 . 7 7 6}$ \\
5 & 2.571 \\
\hline
\end{tabular}

For the sample above, we follow the steps below to calculate CI at confidence level 95\%:

1. Calculate sample mean and standard deviation.

$$
\mu=0.85289, \sigma=0.00301
$$

2. Subtract the confidence level from 1, then divide by two.

$$
\alpha=(10.95) / 2=0.025
$$

3. Look up answers to $d f=4$ and $\alpha=0.025$ in the t-distribution table. The result is 2.776 . 
4. Divide the sample standard deviation by the square root of the sample size $n$.

$$
0.00301 / \sqrt{5}=0.00135
$$

5. Multiply results from last two steps.

$$
2.776 \times 0.00135=0.00374
$$

6. For the lower end of the range, subtract value of the step above from the sample mean.

$$
0.852890 .00374=0.84914
$$

7. For the higher end of the range, add the same value to the sample mean.

$$
0.85289+0.00374=0.85663
$$

From the calculations above, the CI of the availability is from 0.84914 to 0.85663 . 